\title{
Guidelines
}

\section{Prolonged Weaning: S2k Guideline Published by the German Respiratory Society}

\author{
Bernd Schönhofer $^{a} \quad$ Jens Geiseler ${ }^{b}$ Dominic Dellweg ${ }^{c}$ Hans Fuchs $^{d}$ \\ Onnen Moerer ${ }^{e}$ Steffen Weber-Carstens ${ }^{f}$ Michael Westhoff ${ }^{g, h}$ \\ Wolfram Windisch ${ }^{\mathrm{i}}$
}

${ }^{a}$ Klinikum Agnes Karll Krankenhaus, Klinikum Region Hannover, Laatzen, Germany; ${ }^{b}$ Klinikum Vest, Medizinische Klinik IV: Pneumologie, Beatmungs- und Schlafmedizin, Marl, Germany; ${ }^{C}$ Fachkrankenhaus Kloster Grafschaft

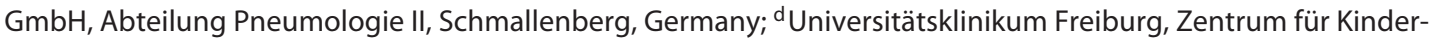

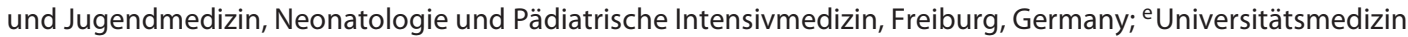
Göttingen, Klinik für Anästhesiologie, Göttingen, Germany; ${ }^{f}$ Charité, Universitätsmedizin Berlin, Klinik für Anästhesiologie mit Schwerpunkt operative Intensivmedizin, Campus Virchow-Klinikum und Campus Mitte,

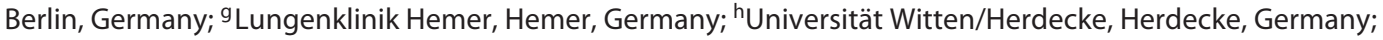
'Lungenklinik, Kliniken der Stadt Köln gGmbH, Universität Witten/Herdecke, Herdecke, Germany

The full version of these Guidelines are available online only, free of charge, at www.karger.com/doi/10.1159/000510085

Prof. Dr. Bernd Schönhofer and Dr. Bernd Schönhofer Klinikum Agnes Karll Krankenhaus Klinikum Region Hannover, Hildesheimer Str. 158 DE-30880 Laatzen (Germany) Bernd.Schoenhofer@t-online.de 


\section{Contents}

\section{Abstract}

\section{Introduction}

\section{Methodology}

2.1. Preamble

2.2. Composition of the Guideline Committee

2.3. Aims of the Guideline

2.4. Guideline Procedures

2.5. Financial Support

\section{Definitions, Epidemiology, and Weaning Categories}

3.1 Stages of Invasive Positive-Pressure Ventilation

3.2. Weaning Process

3.3. Weaning Success and Failure

3.4. Weaning Classification

3.5. Prolonged Weaning versus Prolonged Ventilation

3.6. Definition of the Patient Collective for the Current Guideline

\section{Pathophysiology of Weaning Failure}

4.1. Introduction

4.2. Hypercapnic Respiratory Failure

4.2.1. Respiratory Centre

4.2.2. Neural Drive

4.2.3. Respiratory Muscles

4.2.4. Muscle Overloading

4.2.4.1. Airway Diseases

4.2.4.2. Thoracic Restriction

4.3. Hypoxic Respiratory Failure following Interstitial Lung Disease and Cardiopulmonary Interactions

4.4. Oxygen Transport and Consumption

4.5. Critical-Illness-associated Polyneuropathy and Critical-Illness-associated Myopathy

4.6. Additional Metabolic Aspects

4.7. Special Characteristics of Paediatric Patients

\section{Weaning Strategies}

5.1. Strategies to Prevent Long-Term MV and Prolonged Weaning

5.1.1. Assessment of Weaning Potential

5.1.2. Management of Sedation, Anxiety, Agitation and Pain

5.1.3. Daily Sedation Interruption

5.1.4. Management of Sedation

5.1.5. Anti-Psychotic and Anxiolytic Treatment Concepts 
5.1.5.1. Pathophysiology of Delirium

5.1.5.2. Treatment of Delirium

5.1.6. Infectiological Aspects of Weaning

5.1.7. Spontaneous Breathing Trial (SBT)

5.1.8. Ultrasound Assessment of the Diaphragm

5.1.9. Weaning Protocol

5.1.10. Cuff-Leak Test

5.1.11. Early Mobilisation

5.2. The Significance of Physiotherapy and Measurement of Peak Expiratory Flow in Prolonged Weaning

5.2.2. Measurement of Peak Expiratory Flow in Patients with an Endotracheal Tube or Tracheal Cannula

5.2.3. Combining Different Procedures

5.3. Types of Ventilation Used in Weaning

5.3.1. Introduction

5.3.2. Controlled MV

5.3.3. Assisted MV

5.3.3.1. Pressure-Support Ventilation (PSV)

5.3.4. Combined MV (Synchronized Intermittent Mandatory Ventilation, SIMV)

5.3.5. Adaptive MV

5.3.5.1. Adaptive Support Ventilation (ASV)

5.3.5.2. Neurally Adjusted Ventilatory Assist (NAVA)

5.3.5.3. Proportional Assist Ventilation (PAV)

5.3.6. Hybrid MV

5.3.7. Monitoring

5.3.8. Weaning Concepts Associated with the Use of Various Forms of Ventilation

5.3.8.1. Gradual Reduction of Support

5.3.8.2. Automated Adjustment of Assisted Ventilation (Automated Weaning)

5.3.9. Intermittent Loading by Disconnecting the Ventilator (Spontaneous Breathing) or the Reduction of Pressure Support

5.3.10. Comparison of Different Ventilation Approaches and Weaning Concepts

5.3.11. The Role of Continuous Positive Airway Pressure in Weaning

5.4. The Ventilation Interface

5.4.1. NIV Interfaces

5.4.2. Invasive Ventilation Interfaces

5.4.2.1. Endotracheal Tube

5.4.2.2. Tracheotomy

5.4.2.2.1. Tracheotomy Techniques

5.4.2.2.2. Percutaneous Dilatational Tracheotomy (PDT)

5.4.2.2.3. Surgical Tracheotomy

5.4.2.2.4. When to Perform a Tracheotomy

5.4.3. Management of Tracheal Cannula

5.4.4. Decannulation

5.4.5. The Use of Tracheostomy Retainers

5.4.6. Closure of the Tracheostoma

5.4.6.1. Spontaneous Closure

5.4.6.2. Surgical Closure/Plastic Wound Closure

5.4.7. Complications following Decannulation

5.4.8. Bronchoscopic Monitoring after Decannulation 
5.5. NIV and High-Flow-Oxygen Administration in Difficult-to-Wean Patients and the

Post-Extubation Phase

5.5.1. Criteria for NIV Capacity during the Weaning Process

5.5.1.1. The Use of NIV following Hypercapnic Acute Respiratory Failure (ARF)

5.5.1.2. The Use of NIV following Hypoxemic Acute Respiratory Failure (ARF)

5.5.1.3. The Use of NIV in CRF following Weaning From Long-Term Invasive MV

5.5.2. High-Flow Oxygen for the Prevention of Post-Extubation Failure and Its Use in Weaning

5.6 Prolonged Weaning Strategies

5.6.1. Adjunctive Measures

5.6.1.1. The Influence of Respiratory Drive

5.6.1.1.1. Reduction in Respiratory Drive

5.6.1.1.2. Stimulated Respiratory Drive

5.6.1.2. The Role of Transfusion in Prolonged Weaning

5.6.1.3. Improving Nutritional Status and Metabolism

5.6.1.4. Secretion Management

5.6.1.5. Management of Dysphagia

5.6.1.6. Measures for Improving Diaphragm Function in Prolonged Weaning

5.6.1.6.1. Inspiratory Muscle Training (IMT)

5.6.1.6.2. Respiratory Muscle Support by Indirect and Direct Stimulation of the Diaphragm

5.6.2. Extracorporeal Lung Support Procedures

5.6.3. Sleep on the ICU

5.6.3.1. Significance of Melatonin

5.7. Features of Spinal Cord Transection (SCT)

5.7.1. Pathophysiological Aspects

5.7.2. Special Features of Patients with SCT

5.7.3. Additional Exclusion Criteria for Beginning the Weaning Process

5.7.4. Practical Course of the Weaning Procedure

5.8. Special Aspects in Paediatric Patients

5.8.1. Weaning Protocols

5.8.2. Assessment of Weaning Ability

5.8.3. Sedation and Sedation Protocols

5.8.4. SBTs and the Prediction of Successful Extubation

5.8.5. Types of MV in Weaning

5.8.6. Post-Extubation Stridor

5.8.7. Tracheotomy

5.8.8. Non-Invasive Ventilation (NIV)

5.8.9. High-Flow Nasal Cannula (HFNC)

\section{Infrastructure and Process Organisation for the Care of Patients in Prolonged Weaning: A Continuous Treatment Concept}

6.1. Introduction

6.2. Acute-Care Hospital

6.3. Weaning Units and Weaning Centres

6.3.1. Special Features of Paediatric Patients

6.4. Early Rehabilitation

6.4.1. Special Characteristics of Early Neurological-Neurosurgical Rehabilitation

6.4.2. Special Features of Early Pulmonary Rehabilitation

6.4.2.1. Patient Population

6.4.2.2. Neuromuscular Spontaneous Breathing Capacity in Early Rehabilitation

6.4.2.3. Transition from Pulmonary Early Rehabilitation 
6.5. Management of Transition and Discharge

6.6. Home MV and Follow-Up Care after Prolonged Weaning

6.6.1. Category 3a I: Successful Weaning with Decannulation

6.6.2. Category 3a II: Successful Weaning without Decannulation

6.6.3. Category 3b I: Successful Weaning with Long-Term NIV, without the Need for Supplementary Nursing Care

6.6.4. Category 3b II: Successful Weaning with Long-Term NIV, with the Need for Supplementary Nursing Care

6.6.5. Category 3c I: Unsuccessful Weaning with the Continuation of Invasive MV in the Outpatient Setting

6.6.6. Special Features of Paediatric Patients

6.7. Structural and Procedural Quality Requirements in Prolonged Weaning

6.7.1. Requirements for Weaning Units or Weaning Centres

6.7.2. Number of Treated-Patient Cases

6.7.3. Staffing Requirements

6.7.4. Medical Expertise

6.7.5. Technical Set-Up

6.7.6. Room Facilities

\section{Therapy Decisions for the End-of-Life Phase}

7.1. Issues

7.1.1. Ethical Consideration

7.1.2. The Question of the Purpose of Treatment

7.1.3. Legal Requirements

7.1.4. Ethical Counselling

7.2. Communication

7.2.1. Discussion Conduct

7.3. Finishing MV Treatment

7.3.1. Procedures for Ending Ventilation Therapy

7.3.2. Terminal Respiratory Secretions ("Death Rattle")

7.4. Documentation

7.5. Special Aspects in Paediatric Patients 


\section{Keywords}

End of life - Epidemiology - Mechanical ventilation . Neurorehabilitation · Pathophysiology · Prolonged weaning . Pulmonary rehabilitation

\section{Abstract}

Mechanical ventilation (MV) is an essential part of modern intensive care medicine. MV is performed in patients with severe respiratory failure caused by respiratory muscle insufficiency and/or lung parenchymal disease; that is, when other treatments such as medication, oxygen administration, secretion management, continuous positive airway pressure (CPAP), or nasal high-flow therapy have failed. $\mathrm{MV}$ is required for maintaining gas exchange and allows more time to curatively treat the underlying cause of respiratory failure. In the majority of ventilated patients, liberation or "weaning" from $M V$ is routine, without the occurrence of any major problems. However, approximately $20 \%$ of patients require ongoing MV, despite amelioration of the conditions that precipitated the need for it in the first place. Approximately 40 $50 \%$ of the time spent on MV is required to liberate the patient from the ventilator, a process called "weaning". In addition to acute respiratory failure, numerous factors can influence the duration and success rate of the weaning process; these include age, comorbidities, and conditions and complications acquired during the ICU stay. According to international consensus, "prolonged weaning" is defined as the weaning process in patients who have failed at least 3 weaning attempts, or require more than 7 days of weaning after the first spontaneous breathing trial (SBT). Given that prolonged weaning is a complex process, an interdisciplinary approach is essential for it to be successful. In specialised weaning centres, approximately $50 \%$ of patients with initial weaning failure can be liberated from MV after prolonged weaning. However, the heterogeneity of patients undergoing prolonged weaning precludes the direct comparison of individual centres. Patients with persistent weaning failure either die during the weaning process, or are discharged back to their home or to a long-term care facility with ongoing MV. Urged by the growing importance of prolonged weaning, this Sk2 Guideline was first published in 2014 as an initiative of the German Respiratory Society (DGP), in conjunction with other scientific societies involved in prolonged weaning. The emergence of new research, clinical study findings and registry data, as well as the accumulation of experience in daily practice, have made the revision of this guideline necessary. The following topics are dealt with in the present guideline: Definitions, epidemiology, weaning categories, underlying pathophysiology, prevention of pro-

Prolonged Weaning: S2k Guideline by the German Respiratory Society longed weaning, treatment strategies in prolonged weaning, the weaning unit, discharge from hospital on MV, and recommendations for end-of-life decisions. Special emphasis was placed on the following themes: (1) A new classification of patient sub-groups in prolonged weaning. (2) Important aspects of pulmonary rehabilitation and neurorehabilitation in prolonged weaning. (3) Infrastructure and process organisation in the care of patients in prolonged weaning based on a continuous treatment concept. (4) Changes in therapeutic goals and communication with relatives. Aspects of paediatric weaning are addressed separately within individual chapters. The main aim of the revised guideline was to summarize both current evidence and expert-based knowledge on the topic of "prolonged weaning", and to use this information as a foundation for formulating recommendations related to "prolonged weaning", not only in acute medicine but also in the field of chronic intensive care medicine. The following professionals served as important addressees for this guideline: intensivists, pulmonary medicine specialists, anaesthesiologists, internists, cardiologists, surgeons, neurologists, paediatricians, geriatricians, palliative care clinicians, rehabilitation physicians, intensive/chronic care nurses, physiotherapists, respiratory therapists, speech therapists, medical service of health insurance, and associated ventilator manufacturers.

(c) 2020 S. Karger AG, Basel

\section{Introduction}

The foundation for the global use of mechanical ventilation (MV) with positive airway pressure (positivepressure ventilation) was initially established in the 1950s in the form of negative pressure ventilation. This occurred through the work of the anaesthetist Björn Ibsen, who during the poliomyelitis epidemic was the first to apply this technique on a large scale in patients with respiratory failure $[1,2]$. Nowadays, MV is a central component of modern intensive care medicine and is increasingly being incorporated into hospital wards with other levels of care, as well as in outpatient MV settings [3]. MV is employed in patients who have severe respiratory insufficiency either as the result of respiratory pump dysfunction or due to the failure of the lungs to oxygenate or decarboxylate, and who cannot be sufficiently stabilised through other measures such as supplemental oxygen, positioning therapy, secretion management or CPAP. Furthermore, there are also indications that are primarily non-respiratory (e.g., coma, intoxication) but require the use of MV because of respiratory drive dysfunction, in- 
sufficient defensive reflexes, or impending displacement of the airways $[4,5]$. The primary goals of MV are to stabilise the alveolar gas compartments, ensure a sufficient level of pulmonary gas exchange, and to normalise or minimise the work of breathing. Indeed, the underlying cause of the patient's respiratory failure is treated during the MV period; however, MV therapy often then has to be continued, even when the original indication (e.g., severe pneumonia) has long been rectified. This means that around $40-50 \%$ of an intensive care patient's entire duration of MV corresponds to the liberation from MV ("weaning") [4-8].

In practice, the majority of ventilated patients can be weaned after short period of MV therapy without any significant problems; however, for the remaining patients (approx. 20\%), the weaning phase is markedly extended.

Due to the complexity of the weaning procedure [9], the patient is at risk of permanent weaning failure with subsequent invasive home MV, which for many patients can impose enormous limitations on their quality of life $[10,11]$. This is where specialised weaning centres/units play an important role by averting weaning failure in approximately half the patients [12], a desirable outcome not only in terms of quality of life and patient independence, but also from a financial perspective for the health system.

Long-term home MV is potentially required in the group of patients for whom weaning from invasive $\mathrm{MV}$ is not possible [12]. This situation is exacerbated by demographic growth, further development of operational techniques including modern anaesthetic procedures, advances in intensive care medicine with improved survival of acute diseases, and the increase across all facilities and levels of care in the proportion of often-multimorbid geriatric patients. Therefore, based on the increasing importance of prolonged weaning, this guideline project was established by a joint initiative between the German Respiratory Society (DGP) and other scientific societies involved in this topic.

This is the first revision of the "Prolonged Weaning" guideline that was published in the German language in 2014 [13], and pertains to patients undergoing the prolonged weaning process. The revision was carried out on the basis of the latest research and clinical study findings, new data from patient registries, and the cumulative experience and expertise acquired from daily clinical practice.

As in the 2014 version, statements and recommendations related to paediatric patients will again be discussed separately in individual chapters.
Particular focus was placed on the following topics during the revision process:

- A new classification of patient subgroups in prolonged weaning

- The important aspects of pneumological and neurological rehabilitation in prolonged weaning

- Infrastructure and the organisation of procedures required for patient care in prolonged weaning, particularly in the context of a continuous treatment concept

- Alteration of therapy goals and communication with relatives

In addition, the revised guideline draws on current scientific knowledge and practical importance to discuss topics such as the pathophysiological causes of weaning failure, strategies to avoid prolonged weaning, and therapeutic concepts for weaning.

To close this introductory chapter, we would like to note that the Federal Social Court (BSG) of Germany recently declared that an adaptation (German: "Gewöhnung") to the respirator is a prerequisite for weaning ("Entwöhnung") to be able to occur (Reference number: B1 KR 18/17 R; 19th December, 2017) [14]. All societies involved in the preparation of this guideline consider that this BSG-derived link between adaptation and weaning - which, incidentally, has been deduced from an incorrect interpretation of the term "weaning" - is not technically applicable and hence incorrect. The expression "weaning from ventilation" is actually meant to describe the "liberation" from ventilation, which is an internationally established term.

This conceptual misjudgement of a causal chain from "adaptation to weaning" is based on the wrong assumption that in this context, there is a biological change of state like that occurring when weaning from breast milk or a particular substance dependency. However, this is not the case with MV, regardless of the ventilation interface (invasive/non-invasive) used. Pathophysiological adaptation in the conventional sense occurs neither during continuous nor during intermittent ventilation; rather, it is an acute gas exchange disorder and/or weakening or overloading of the respiratory muscles that represent the initial indication for ventilation. The impairment incurred leads to an acute dependency on a life-sustaining ventilator, which ensures survival during the respiratory crisis. The aim should always be to finish this state of MV as quickly as possible. The duration of the obligatory period of $\mathrm{MV}$ is determined by the pathophysiological changes described above, rather than by the time it takes to "adapt" to the ventilator. Therefore, specification of the minimum amount of time required on MV before wean- 
ing becomes necessary is also not objectively justifiable. In summary, the assumption that an "adaptation - weaning" sequence of ventilation is necessary is factually incorrect and should be replaced by a "dependence - weaning" sequence of ventilation.

\section{Methodology}

\subsection{Preamble}

The previous version of this guideline for prolonged weaning was published in 2014 [13]. However, based on the research findings, clinical study outcomes and registry data that have since emerged, as well as the experience and expertise that have been acquired from daily clinical practice, a revision of the 2014 guideline was deemed necessary. The participating scientific societies (see title page) have therefore collaborated to create an updated version of the interdisciplinary Prolonged Weaning Guideline, thereby replacing the previous guideline as the latest valid version [13].

\subsection{Composition of the Guideline Committee}

The following people were named as representatives (in alphabetical order) by their respective scientific societies and institutions:

Johannes Bickenbach (DGAI: German Society for Anaesthesiology and Intensive Care Medicine), Stephan Braune (DGIIN: German Society for Internal Intensive Care Medicine and Emergency Medicine), Dominic Dellweg (DGP-Pneu: German Respiratory Society), Christian Dohmen (DGN: German Society for Neurology), Erich Donauer (DGNC: German Society for Neurosurgery), Rolf Dubb (DFG: German Society for Specialist Nurse Care and Functional Services), Markus Ferrari (DGK: German Society for Cardiology), Hans Fuchs (DGPI: German Society for Neonatology and Pediatric Intensive Care Medicine), Jens Geiseler (DGP-Pneu: German Respiratory Society), Dorothea Heidler (dbl: German Federal Association for Speech Therapy), Hans Jürgen Heppner (DGG: German Society for Geriatrics), Sven Hirschfeld-Araujo (DMPG: German Medical Society for Paraplegiology), Uwe Janssens (DGK: German Society for Cardiology), Thomas Jehser (DGP-Pall: German Society for Palliative Medicine), Erich Kilger (DGAI: German Society for Anesthesia and Intensive Care Medicine), Silke Klarmann (VK: German Association for Physiotherapy), Andreas Markewitz (DGTHG: German Society for Thoracic, Cardiac and Vascular Surgery), Onnen Mörer (DGAI: German Society for Anesthesia and
Intensive Care Medicine), Thomas Nicolai (DGPI: German Society for Neonatology and Pediatric Intensive Care Medicine), Marcus Pohl (DGNR: German Society for Neurorehabilitation), Christian Putensen (DIVI: German Interdisciplinary Association for Intensive Care and Emergency Medicine), Jens D. Rollnik (DGNR: German Society for Neurorehabilitation), Simone Rosseau (DIGAB: German Interdisciplinary Society for Out-ofHospital Ventilation), Bernd Schönhofer (DGP-Pneu: German Respiratory Society), Dierk Schreiter (DGCH: German Society for Surgery), Steffen Weber-Carstens (DGAI: German Society for Anesthesia and Intensive Care Medicine), Arved Weimann (DGEM: German Society for Nutritional Medicine), Michael Westhoff (DGPPneu: German Respiratory Society), Wolfram Windisch (DGP-Pneu: German Respiratory Society), Matthias Wittstock (GNI: German Society for Neuro-Intensive and Emergency Medicine).

\subsection{Aims of the Guideline}

This guideline aims to provide consensus-based statements regarding the underlying pathophysiology, diagnostic approaches, and therapeutic strategies associated with patients undergoing prolonged weaning. It applies to hospital-based physicians as well as other professionals who are involved in the care of patients with prolonged weaning. This document should also serve as a useful guide for those people and organisations who are indirectly associated with the topic of prolonged weaning.

\subsection{Guideline Procedures}

The creation of this guideline with an S2k classification level was carried out in accordance with AWMF (Association of Scientific Medical Societies) criteria to provide guideline users with evidence-based criteria for rational decision-making and good clinical practice [15].

For the topic of management in prolonged weaning, important questions related to the content of the 2014 guideline were identified by the guideline committee members, and recommendations or statements were then formulated to address these questions. During the course of establishing the current guideline, these questions and recommendations were extensively discussed within the framework of 3 consensus conferences. This led to the formulation of 55 recommendations and 5 quality indicators for the revised guideline. In order to reach the largest target group possible, the guideline includes recommendations not only for adults, but also for children. Furthermore, it contains a series of background texts that were 
similarly discussed and updated by the guideline committee; these sections serve to deepen the reader's understanding of the topic and promote better handling of the recommendations and statements.

Prior to starting the revision process, the guideline committee gathered on 2 separate occasions in 2016, where members were distributed amongst topic-based work groups, each of whom was then commissioned to carry out a specific set of tasks. Based on the special expertise of the selected authors and the amount of evidence available, the work groups then reviewed the current recommendations and statements, and rewrote them if necessary. The background texts that supplement the recommendation and statements were also either updated or rewritten. A literature search in PubMed was also performed on a chapter-specific basis using keywords entered by the authors, and the results were made available to the guideline authors through the Scientific Guideline Manager database (ILF: Lung Research Institute, Berlin), in order to facilitate the process of updating the guideline content. In addition, literature citations from the previous version of the guideline, as well as those from other guidelines and current studies, were taken into account during the consensus process, provided they were relevant to the content of the current guideline.

The manuscript that arose from this process was then sent to all conference participants prior to the 3 consensus conferences. Under the direction of the AWMF moderator (PD Dr. H. Sitter), the recommendations were extensively discussed, agreed upon and revised in a nominal group process, which also involved specialists from additional scientific societies and organisations with expertise in the field of prolonged weaning. In line with the resolutions of the consensus conferences, a list of specific and well-reasoned adjustments to the manuscript was proposed, and each work group was commissioned to implement these changes accordingly. Following an intensive revision process, the manuscript was sent back to all participants. Based on the final round of feedback, the manuscript was revised by the editorial team and the entire list of references was compiled. The guideline text that was passed during the consensus conferences was then forwarded to the board members of the leading and participating societies and institutions for discussion, comments and approval, all while allowing a sufficient time frame. Following the incorporation of amendments proposed by the board members, the guideline was judged positively and approved.

Further information can be found in the guideline report that is published on the AWMF Website [15].

\subsection{Financial Support}

The preparation of this guideline by the participating societies was not supported by any third-party funds. Organisational support as well as assistance with the literature search was provided by the Lung Research Institute (ILF). All work group members worked exclusively on a voluntary basis, without the exertion of influence from external parties.

\section{Definitions, Epidemiology, and Weaning Categories}

\subsection{Stages of Invasive Positive-Pressure Ventilation}

The definitions and classifications described in the current guideline are not only based on previously published guidelines for acute and chronic respiratory failure $[3,16]$, but are also derived from the findings of an 11 -member international board representing 5 international medical societies (European Respiratory Society [ERS], American Thoracic Society [ATS], European Society of Intensive Care Medicine [ESICM], Society of Critical Care Medicine [SCCM] and Société de Réanimation de Langue Française [SRLF]) [17], which are referred to in the text as necessary.

According to the board's findings, positive-pressure ventilation of the lungs via an endotracheal tube is characterised by 6 stages (Fig. 1) [17]:

1. Treatment of acute respiratory failure.

2. The treating physician contemplates for the first time that the patient could be ready to enter the weaning process.

3. Initiation of daily tests (e.g., rapid shallow breathing index, RSBI) to establish "readiness to wean", thereby confirming or rejecting the assumption that the patient is eligible for weaning.

4. Spontaneous breathing trial (SBT).

5. Extubation (or decannulation).

6. Reintubation (or recannulation), if necessary.

In accordance with the content of the current guideline, stages 5 and 6 have been modified so that decannulation is mentioned instead of extubation, and recannulation is mentioned instead of reintubation.

\subsection{Weaning Process}

The actual weaning process begins at stage 4 with the SBT, and occupies around $40-50 \%$ of the overall MV time [4, 6-8]. The correct timing for commencing stage 4 (SBT) and stage 5 (extubation/decannulation) is critical for prognosis. On one hand, if the artificial airway is re- 


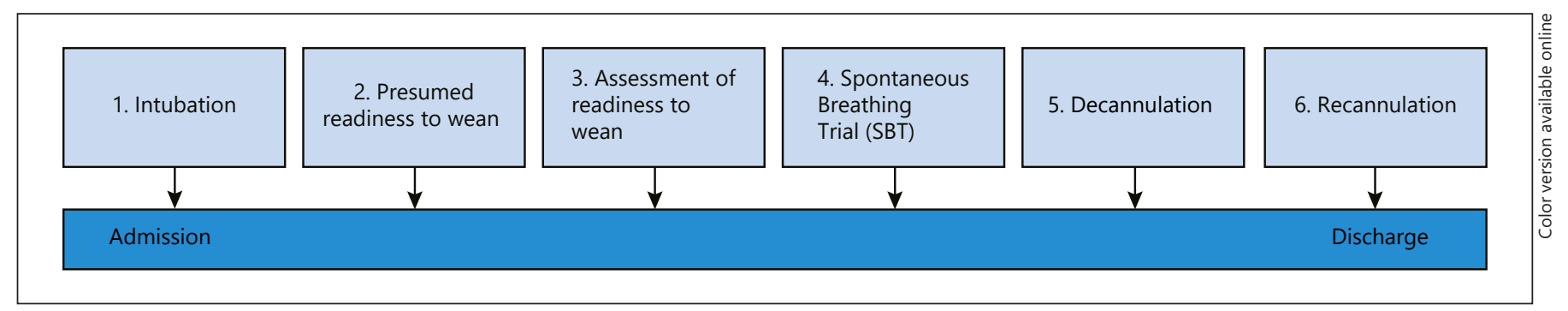

Fig. 1. Schematic diagram of the different stages of invasive mechanical ventilation [17].

moved too early and the patient requires subsequent reintubation/recannulation (stage 6), this is accompanied by an increased risk of nosocomial pneumonia and an extended stay in the intensive care unit (ICU) [18-20]. On the other hand, if weaning is implemented too late, there is an increased complication rate associated with extended periods of MV [21-23]. Indeed, both of these scenarios are associated with a substantially increased risk of mortality. This is where it is important to recognise that delayed anticipation of a patient's readiness to wean, as well as an overdue assessment of the defined criteria for establishing this readiness (ventilation stages 2 and 3, prior to actual weaning), already represent common reasons for why weaning therapy is unnecessarily delayed [17]. The fact that extubation is frequently delayed is also documented by the observation that accidental self-extubation does not result in reintubation in almost $50 \%$ of cases [24]. The incidence of unplanned extubation ranges between 0.3 and $16 \%$, depending on the type of examination, whereby $83 \%$ of them are actively carried out by the patient, and $17 \%$ occur unintentionally [25].

\subsection{Weaning Success and Failure}

According to the definition provided by the 2007 International Task Force, weaning success is established when a patient who has just been extubated does not require ventilatory support for at least $48 \mathrm{~h}$ after the extubation procedure [17]. Accordingly, weaning failure is characterised by: (1) an unsuccessful SBT, (2) reintubation/ recannulation and/or the resumption of $\mathrm{MV}$, or (3) death within 48 hours of extubation [17]. It was already the case at the beginning of the 1990s that weaning failure had been defined as the permanent (continuous or intermittent) need for ventilatory support, whereby both invasive (endotracheal tube, tracheal tube) and non-invasive ventilatory approaches were included in the definition [26]. In the meantime, non-invasive ventilation (NIV) has become firmly established in the weaning process [27] where it is recognised as playing a role in improving prognosis in patients with primary weaning failure, particularly when adequate spontaneous breathing is not possible (i.e., failed SBT), but extubation/decannulation with subsequent NIV takes place nonetheless [27]. Moreover, NIV has favourable effects in patients with secondary weaning failure - that is, primarily sufficient spontaneous breathing (successful SBT) and successful extubation when high-risk patients (e.g., chronic respiratory failure, cardiac insufficiency, older age) in this situation develop respiratory insufficiency [28-31]. According to the definitions mentioned above, a patient who is successfully ventilated by NIV following extubation or decannulation, and who is transferred with NIV to the general ward or can even be discharged home, is not actually weaned from ventilation. In a large cohort study of 403 patients who had undergone invasive ventilation for $>2$ weeks and were transferred to a specialised weaning centre explicitly for the purposes of weaning, Schönhofer and colleagues demonstrated that among the patients discharged home, a third of them still continued long-term NIV in the home setting [12]. This emphasises that it is not always possible to separate the use of NIV to support extubation/decannulation on one side, and treat chronic respiratory failure on the other. The International Task Force thus designates patients who are extubated/decannulated, but still require NIV support, to an intermediate category known as "weaning in progress" [17].

Given the complexity of this topic, a clearer definition is needed. Since "weaned" and "not weaned" are generally the only terms used, it remains unclear whether "weaned" corresponds to the cessation of ventilation or to decannulation. However, making a distinction between "complete weaning" and "weaning from invasive ventilation" is not practical in terms of language use. Nonetheless, the term "weaning" is strictly coupled to the term MV. For this reason, the revised guideline defines successful weaning as the complete cessation of invasive 
MV. According to this definition, patients undergoing prolonged weaning are successfully weaned when, following completion of the weaning process, they either continue to be NIV-dependent in an outpatient setting, or they no longer require MV but for various reasons remain tracheotomised (e.g., severe swallowing disorder, high-grade tracheal stenosis); this particular group generally requires intensive nursing care in the outpatient setting (see Chapter 6.6). The current revised classification obviously still takes into account the differences amongst patients in NIV dependency and decannulation status (see Chapter 3.6). In this context, the classification of prolonged weaning will be further modified and differentiated in the current revised guideline.

\subsection{Weaning Classification}

The International Task Force categorises the weaning process into 3 groups (Table 1) [17]. By referring to earlier data, it is estimated that up to $70 \%$ of weaning patients are allocated to Group 1 and 30\% are distributed across Groups 2 and 3 [22, 24]. An Austrian-based multicentre study demonstrated that in a group of 257 intubated patients that had begun the weaning process and had no cases of self-extubation, 59, 26 and 14\% of patients were allocated to Groups 1, 2 and 3, respectively, using the above-mentioned weaning classification system [32]. The respective ICU mortality rates for Groups 1, 2 and 3 stood at $3 \%, 1 \%$ and $22 \%$, while the respective hospital mortality rates were 13, 22 and $32 \%$. Therefore, when compared to the mortality rate of Group 1 (simple weaning), there was a statistically significant increase in mortality for patients in Group 3 (prolonged weaning), but not for those in Group 2 (difficult weaning). Accordingly, the rate of successful (complete) weaning (see above) was reduced in the prolonged weaning group (Group 3, 74\%), but not the difficult weaning group (98\%, Group 2), when compared to that of the simple weaning group (99\%, Group 1). The median duration of the entire period of MV was 0.5 days (Group 1), 2.9 days (Group 2) and 10 days (Group 3) [32]. In summary, longer periods of $\mathrm{MV}$ and longer hospital stays existed for patients in the difficult weaning group (Group 2), whereas prognosis was restricted for patients from the prolonged weaning group (Group 3) in comparison to that for simple weaning patients (Group 1) [33].

As an alternative to the international weaning classification outlined above, a new classification based on a multinational prospective observational study was proposed in March 2017 (WIND study = Weaning according to a New Definition) [34]. The impetus for the study was
Table 1. International weaning classification [17]

\begin{tabular}{lll}
\hline Group & Category & Definition \\
\hline 1 & $\begin{array}{l}\text { Simple } \\
\text { weaning }\end{array}$ & $\begin{array}{l}\text { Patients who proceed from initiation of } \\
\text { weaning to successful extubation on the first } \\
\text { attempt without difficulty }\end{array}$ \\
\hline 2 & $\begin{array}{l}\text { Difficult } \\
\text { weaning }\end{array}$ & $\begin{array}{l}\text { Patients who fail the initial weaning attempt } \\
\text { and require up to 3 SBTs, or as long as 7 } \\
\text { days from the first SBT to achieve successful } \\
\text { weaning }\end{array}$ \\
\hline 3 & $\begin{array}{l}\text { Prolonged } \\
\text { weaning }\end{array}$ & $\begin{array}{l}\text { Patients who fail at least 3 weaning } \\
\text { attempts, or require 7 days of weaning after } \\
\text { the first SBT }\end{array}$ \\
\hline
\end{tabular}

SBT, spontaneous breathing trial.

based on the fact that many patients could not be classified according to the criteria published by the International Consensus Conference. Indeed, these criteria are mandatorily based on the implementation of SBTs, which is not always possible to carry out in clinical practice. Furthermore, these criteria are based on successful weaning, which does not always correspond to daily clinical practice. Patients who do not attempt weaning at all are also not taken into account. Ultimately, rather than being based on clinical study findings, the International Consensus Conference criteria were primarily derived from expert opinion. Accordingly, studies have retrospectively demonstrated that there is no prognostic difference between simple and difficult weaning, with no distinction between intubated and tracheotomised patients. In fact, in almost $50 \%$ of cases classifications of the WIND study patient cohort was not possible when the International Consensus Conference Criteria were used [34].

For this reason, the classification system in the WIND study is primarily based on a separation attempt, regardless of whether or not a reduction in ventilation parameters has taken place (see Table 2). To this end, definitions are given for both the separation attempt and weaning success, not only for intubated patients, but also for tracheotomised patients. Accordingly, almost all patients from the WIND study were able to be classified according to the newly established criteria.

The authors of the WIND study have lodged a claim to have their new criteria replace the current ones. It should be noted, however, that the WIND study was conducted in 36 ICUs rather than actual weaning units, where patients are treated with very long periods of MV (see Chapter 3.5). 
Table 2. Classification of weaning categories according to the WIND study [34]

Group 0 - "no weaning": patients who have not previously experienced a separation attempt

Group 1 - "short weaning": the first attempt resulted in termination of the weaning process within 1 day (successful separation or early death)

Group 2 - "difficult weaning": weaning was completed after more than 1 day but in $<1$ week after the first separation attempt (successful separation or death)

Group 3 - "prolonged weaning": weaning was still not terminated (by successful separation or death) 7 days after the first separation attempt

Group $3 \mathrm{a}$ - prolonged weaning led to successful weaning 7 days or more after the first attempt

Group $3 b$ - prolonged weaning was not successful

To this end, the average treatment duration on the ICU was a mere 6 days, whereby only $1.4 \%$ of the patients remained invasively ventilated via a tracheal cannula, while the number of patients with NIV was also very low. For this reason, the WIND Criteria cannot be considered as a better alternative to the International Consensus Conference Criteria. Moreover, it remains questionable whether the criteria for the separation attempt actually hold true for the patient collective of this guideline. Therefore, this guideline revision upholds the criteria described by the International Consensus Conference [17]. Further investigations that address the classification of weaning patients in the patient cohort described here remain to be carried out.

\subsection{Prolonged Weaning versus Prolonged Ventilation}

While the International Task Force clearly defines prolonged weaning and this definition has been adopted by the current guideline, the terms prolonged weaning and long-term MV are not clearly distinguished. According to the literature, a minimum MV period of 2-3 weeks is required for allocation to the long-term MV category $[33,35]$. However, it is important to note that a patient who requires more than 2 weeks of invasive MV due to a severe underlying condition won't necessarily have difficulties in weaning from the respirator after the underlying condition has successfully been treated. This is the kind of situation in which the terms "long-term MV" and "prolonged weaning" are intermingled. In the above-cited study by Schönhofer et al., long-term ventilation was described as being established when it continued for more than 2 weeks, and at least 2 failed attempts at weaning had taken place [12]. In other publications, the term prolonged ventilation is used when MV takes place over a period of at least 3 weeks [36].

This guideline therefore specifically pertains to patients whose extended requirement for $\mathrm{MV}$ is based on the difficulties they have with weaning. A definition of the term "long-term MV" will therefore not be explicitly undertaken in this guideline.

Nonetheless, reference should be made here to the current guidelines for the indication, organisation and undertaking of home MV when long-term MV is performed in the outpatient setting [3].

\subsection{Definition of the Patient Collective for the Current Guideline}

According to international weaning classification (see Chapter 3.4), prolonged weaning is defined as weaning that is only successful either after 3 failed SBTs, or after more than 7 days following the first failed SBT [17]. However, given the very broad formulation of the definition, this particular group of patients appears to be rather heterogenous. Indeed, according to an Austrian outcome study on prolonged weaning, the median duration of successful weaning was 10 days [32]. In contrast to this, Schönhofer and colleagues reported that patients from various external hospitals who had failed weaning were referred to a specialised weaning centre only after an average of 33 days of invasive ventilation [12]. Amongst these patients, around $20 \%$ were able to be weaned without further support from the respirator, while about $30 \%$ were provided with long-term NIV due to chronic respiratory insufficiency; approximately $30 \%$ of patients were definitively unable to be weaned from the respirator, and the remaining 20\% died whilst still in hospital. Additional data show that particularly in patients with pre-existing respiratory pump insufficiency, the average time that lapses from the point of intubation to the first SBT is one month, and that these patients have a low weaning rate [37]. Data collected from the WeanNet Registry between 2011 and 2015 also show that patients who were previously transferred to a specialised weaning unit spend an average of 23 days on the ICU [38]. In addition, there is an increasing number of patients who are unable to be decannulated, despite having undergone a successful SBT. In particular, patients with persistent swallowing disorders or those requiring ongoing tracheal suction despite being liberated from ventilation - represent a separate patient cohort after prolonged weaning that has so far received little consideration. 
Table 3. Subgroups of prolonged weaning patients according to the current guideline's definition

\begin{tabular}{ll}
\hline $\begin{array}{l}\text { 3a. Successful prolonged weaning from invasive } M V \text { without the need for subsequent long-term NIV } \\
\text { 3a I Patients with extubation/ }\end{array}$ & $\begin{array}{l}\text { Successful weaning from invasive MV with extubation/decannulation only after at least } 3 \text { failed SBTs, } \\
\text { or after a MV period of }>7 \text { days following the first failed SBT without home mechanical ventilation }\end{array}$ \\
\hline 3a II Patients without decannulation & $\begin{array}{l}\text { Successful weaning from invasive MV after at least } 3 \text { failed SBTs or after a MV period of }>7 \text { days } \\
\text { following the first failed SBT, without decannulation }\end{array}$
\end{tabular}

3b. Successful prolonged weaning from invasive $M V$ with the continuation of NIV

3b I Patients with long-term NIV without Successful weaning from invasive MV with extubation/decannulation only after at least 3 failed SBTs, the need for additional nursing care or after a ventilation period of $>7$ days following the first failed SBT, and only with the use of NIV, which, following conclusion of the weaning process, is continued in patients with independence in a home MV setting

3b II Patients with long-term NIV with the Successful weaning from invasive MV with extubation/decannulation only after at least 3 failed SBTs, need for additional nursing care or after a ventilation period of $>7$ days following the first failed SBT, and only with the use of NIV, which, following conclusion of the weaning process, is continued in a home MV setting and requires further nursing support

3c. Failed weaning from invasive $M V$

3 c I Patients with continued invasive ventilation in an outpatient setting

3c II Death

Failed weaning with the continuation of invasive ventilation via a tracheostoma in a home MV setting following conclusion of the weaning process

Failed weaning with death of the patient in hospital

SBT, spontaneous breathing trial; NIV, non-invasive ventilation.

Findings emerging from Germany suggest that the increase in invasive and non-invasive ventilation in the home environment that also arises as a consequence of prolonged weaning processes has also seen a rapid growth in the in-hospital treatment of patients undergoing longterm MV [39]. To this end, in 2016 alone, 86,117 patients were encoded with the OPS Z99.1 (Long-term Ventilator Dependency) within the course of their respective hospital stays.

Indeed, the specialised weaning centres in Germany mainly deal with patients who are either severely ill or can often no longer even be weaned. The duration of MV can sometimes reach well above 7 days after the first SBT, and long-term home mechanical ventilation (HMV) is carried out in a large proportion of patients following discharge from hospital $[3,37]$. It is therefore likely that the group 3 (prolonged weaning) [17] defined by international weaning classification comprises very different patients with widely-varying prognostic factors. Therefore, the current guideline further distinguishes this prolonged weaning group into 3 subgroups ([Table 3]) which, following primary allocation to the internationally recognised weaning group 3, arise based on the further course of weaning:

The group described as subgroup $3 \mathrm{~b}$ in the first edition of this guideline (prolonged weaning with NIV) can be further differentiated as follows (also see Chapter 6.6):
- Patients with temporary NIV: Following extubation/ decannulation of these patients, NIV is only carried out in the hospital until spontaneous breathing becomes possible for several days, without the manifestation of hypercapnia (complete weaning). This particular group is very heterogenous. In isolated cases, ventilatory insufficiency can reoccur following discharge from hospital, with the indication for temporary or lasting NIV. Therefore, a closely-meshed, pulmonological follow-up of these patients on the hospital ward or in an outpatient clinic may be necessary. Regular follow-up examinations should also be performed in successfully weaned patients in view of the potential emergence of chronic respiratory failure [3].

- Patients with direct transition into home-based NIV: These patients are led directly into the home setting with NIV [3], whereby the type of clinical improvement can occasionally occur - even after several months - that renders the continuation of non-invasive home MV unnecessary, for example, in the case of significant weight loss in severe obesity or of a slowly progressing improvement in critical-illness polyneuropathy. The complete cessation of MV in the clinic is, however, initially not possible and absolutely not intended, while weaning from invasive MV is. This category generally deals with patients in whom chronic 
respiratory failure continues to exist, despite being successfully treated for a preceding acute deterioration in their condition, e.g., COPD patients with an acute exacerbation on the basis of pre-existing chronic respiratory failure. A high level of NIV dependency can exist in this situation, which means that the withdrawal of NIV is highly likely to be accompanied by acute respiratory deterioration within a short period of time, possibly with the requirement for renewed invasive MV. This is particularly illustrated by a special group comprising paraplegic patients. Here, weaning can occasionally be achieved according to the above-described definition, when the patient is transferred from invasive MV to NIV. This, however, can lead to 24-h NIV dependency, such that weaning from the ventilator has not actually taken place at all during the transition from invasive MV to NIV. Due to the special nature of this group, more details can be found in Chapter 5.7; however, it should be noted that an extremely high level of NIV dependency can also essentially exist in other types of patients, especially those with neuromuscular diseases or respiratory drive disorders. A high dependency on NIV may then be accompanied by an additional need for treatment or support, some of which takes place in the clinic. In such cases, long-term NIV must be deemed as a means of life-support. At the same time, the autonomy of these patients may be considerably compromised, such that they generally require a high level of support. Further details regarding the ongoing treatment and care requirements of these patients can be found in Chapter 6.6.4 of this guideline.

- In contrast to this, a minimal dependency on NIV can also exist (see Chapter 6.6.3), whereby NIV is primarily implemented to treat symptoms of chronic hypoventilation and improve long-term prognosis [3]. These patients therefore have no further need for additional treatment and support (see above). While these patients could indeed initially complete the formal weaning process, the introduction of long-term NIV is generally indicated [3]. Therefore, it should always be determined in prolonged weaning patients whether an indication for long-term NIV exists, even if extubation/decannulation initially appears successful. In this respect, an indication for the elective implementation of non-invasive home MV should always be determined early on in patients who are at high risk of ventilator-dependent exacerbation of their COPD [3], particularly those who have previously been hospitalised due to acute respiratory failure.

Prolonged Weaning: S2k Guideline by the German Respiratory Society
It is also important to note at this point that it is very difficult to perform an assessment of the patient's prognosis based on the further differentiation of weaning group 3 (prolonged weaning) into the subgroups $3 a, 3 b$ and $3 c$, as outlined in the first version of this guideline. In essence, there are substantial co-morbidities that often contribute considerably to the prognosis, with severe cardiovascular primary diseases, vascular diseases, accompanying lung diseases, neurological deficits, diseases that compromise the immune system, kidney diseases, liver cirrhosis and diabetes mellitus being those at the forefront [38]. In recent years, there has been an increase in both the degree of comorbidity severity and the age of patients undergoing prolonged weaning. Both factors are substantially responsible for the prognosis of these patients having worsened in weaning centres, despite the treating physicians and therapists becoming increasingly experienced [38]. Moreover, even successfully weaned patients with normocapnia can require MV once again, or even die, often with weeks, especially when extremely harmful co-morbidities exist [12]. This has led to the use of the term "unstably weaned" for these patients, even when longer survival is possible in isolated cases [12].

It should also be mentioned that NIV can already be implemented early on in the weaning process, and not just after either at least 3 unsuccessful SBTs, or a ventilation phase of more that 7 days after the first failed SBT, as defined for group 3 of prolonged weaning patients [17]. This was exemplified in a study by Nava and colleagues where patients with exacerbated COPD who had undergone 48 hours of invasive MV could already be extubated after just one failed attempt at an SBT and continue to be ventilated via NIV [28]. However, since these patients do not correspond to any of the defined groups, they will not be addressed in this guideline. This also pertains to other severely ill patients who are better represented in the context of acute medicine rather than prolonged weaning. Such patients include those with extracorporeal circulatory systems that therefore have the goal of reducing the duration of MV, or avoiding it completely.

The current guideline primarily focuses on patients in the internationally-defined weaning group 3 [17]. The prolonged weaning subgroups (Table 3 ) that have been specifically redefined in this guideline are discussed with particular reference to the different medical approaches to weaning, ethical considerations, and prognostically relevant factors, not to mention the organisational structures required for optimal weaning. The guideline is rel- 
evant to patients with severe respiratory dysfunction, whose comorbidities often have a confounding influence on the weaning process.

Accordingly, the above-defined subgroups 3a (prolonged weaning without NIV), $3 \mathrm{~b}$ (prolonged weaning with NIV) and $3 c$ (failed weaning) will be further differentiated in the current guideline, where it will be clearly specified for each category whether or not weaning is successful (see Chapter 3.4). It is also specified whether decannulation was possible, which should be considered independently to the success or failure of the weaning. To this end, it will be emphasised for each group that important conditions persist after the conclusion of the weaning process (cannulation, long-term NIV, care requirements) that need to be considered. This approach particularly takes the highly heterogenous patient cohort in prolonged weaning into account (e.g., neuromuscular disease patients versus COPD). Here, a severity grading is less relevant to the weaning categorisation process (although this is partly present) than a grouping, which bears much more importance for ongoing treatment strategies (compromised or lack of patient autonomy, increased need for nursing care, follow-up examinations).

It should also be noted that temporary NIV therapy during the transition phase that follows decannulation is not really represented by any particular subgroup when it is not continued as long-term NIV in an outpatient setting. Since transient NIV (what used to be known as $3 \mathrm{~b}$ ) represents just one of that many measures that can be taken to successfully wean patients from invasive MV, these patients are now classified into group $3 \mathrm{a}$.

Another important fact is that according to the new definition, a significantly reduced level of patient autonomy and a stronger need for care support has to be expected for groups $3 \mathrm{a}$ II, $3 \mathrm{~b}$ II and $3 \mathrm{cI}$ than that for the groups $3 \mathrm{a}$ I and $3 \mathrm{~b}$ I (Table 3 ).

\section{Pathophysiology of Weaning Failure}

\subsection{Introduction}

Weaning failure is characterised by the need for either continuing long-term $\mathrm{MV}$, or resuming it for a short period, due to an inadequate spontaneous breathing ability. In this situation there is an imbalance between ventilatory demand and ventilatory capacity, which is almost always due to overloading of the respiratory muscles (respiratory pump), and thus rarely attributed to direct malfunctioning of the respiratory centre [40-43]. The main outcome of this situation is hypercapnic respiratory fail- ure. However, weaning failure can also occur in patients who have a normal $\mathrm{PaCO}_{2}$ during $\mathrm{MV}$ and a hypoxemic gas exchange disorder in the foreground. In cases of advanced primary disease, such as pulmonary fibrosis, patients can often no longer be weaned; this is because the ventilatory demand without $\mathrm{MV}$ is so large (due to the reduced area for gas exchange) that in addition to hypoxia, hypercapnic respiratory failure can result from volume overloading of the respiratory pump. However, if the factors leading to gas exchange dysfunction that requires MV are reversible (e.g., acute respiratory distress syndrome [ARDS] or pneumonia), the lung parenchyma can recover to the point where the potential for successful weaning exists. Since prolonged weaning is often multifactorial, combinations of conditions including increased load (obstruction and hyperinflation in COPD), muscle failure (e.g., critical-illness myopathy [CIM] and -polyneuropathy [CIP], diabetic neuropathy), gas exchange disorders and/or cardiac failure can exist, and even negatively interact with each other.

\subsection{Hypercapnic Respiratory Failure}

There are 2 basic mechanisms underlying the type of hypercapnic respiratory failure that arises from respiratory pump failure and has hypercapnia as the leading parameter: (1) Failure of the respiratory muscles, which are then unable to provide a sufficient level of ventilation for the maintenance of gas exchange, and (2) (sub)-acute overloading of the remaining muscle performance capacity through an increased respiratory load due to various illnesses. The extent of gas exchange and ventilatory effort required are determined by the patient's current clinical situation (e.g. infection, cardiac and pulmonary function, muscle strength etc.), as well as a pre-existing gas exchange disorder or impairment of the respiratory muscle pump. All factors can contribute to weaning failure, either in combination, or on an individual basis. To better address the complexity of situations occurring in individual cases, each clinical picture is considered on the basis of its pathophysiological causes (i.e., muscle failure and muscle overloading), rather than using the common approach of discussing cases on an isolated basis. Table 4 summarises the factors linked to each cause of respiratory pump failure, with examples for each.

\subsubsection{Respiratory Centre}

Adequate breathing is only possible with a proper regulatory system for respiration. Disruption to this system can have a number of causes [44-48], although amongst these, the loss of central respiratory drive counts as a rare 
Table 4. Pathophysiology and possible causes of respiratory pump failure

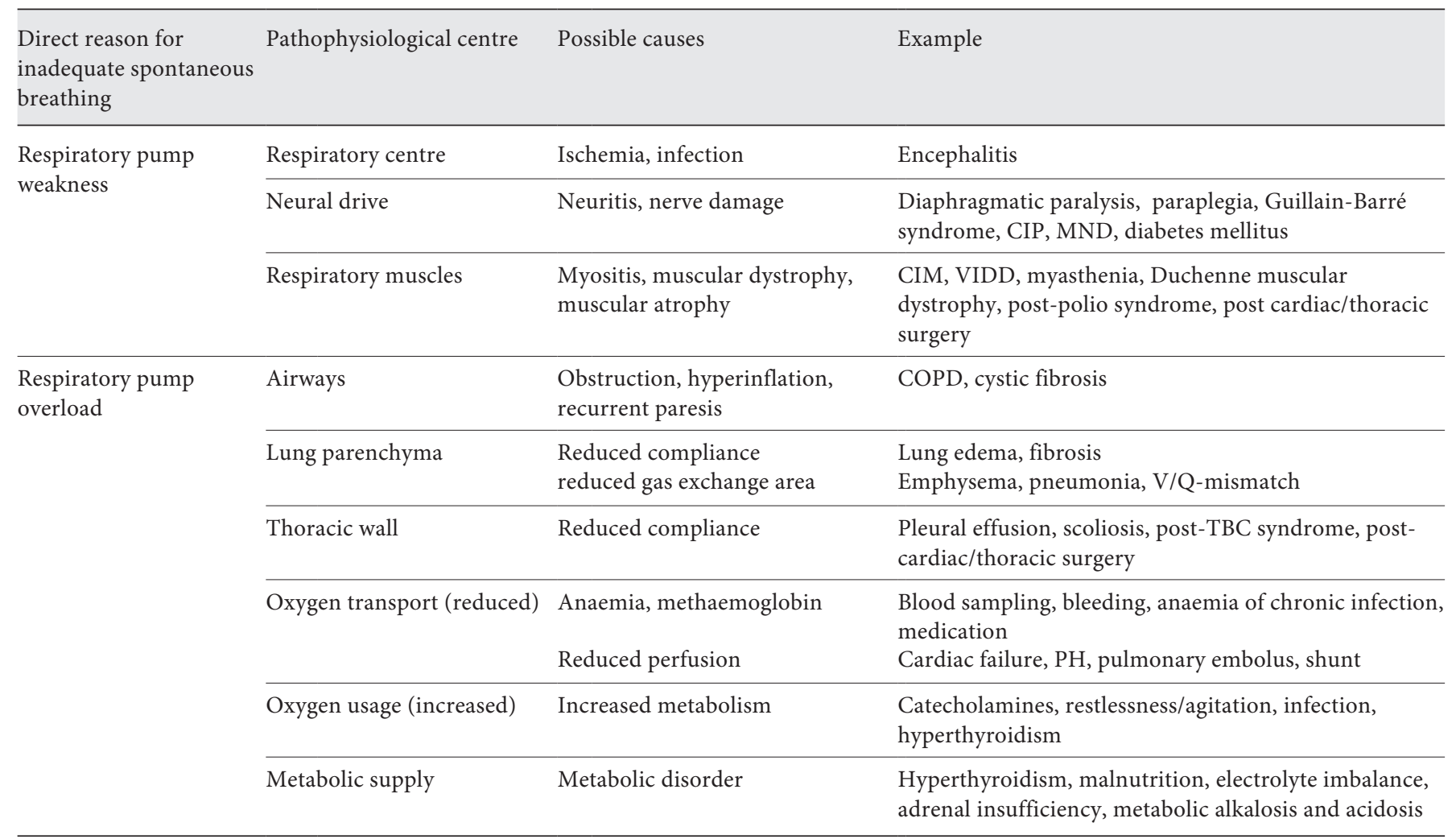

CIP, critical illness polyneuropathy; MND, motor neuron disease; CIM, critical illness myopathy; VIDD, ventilator-induced diaphragmatic dysfunction; $\mathrm{PH}$, pulmonary hypertension; post-TBC syndrome, long-term effects of pulmonary tuberculosis.

cause of weaning failure. The most prominent factors that curb respiratory drive (frequency and depth) are overdoses of medication (sedatives, narcotics, anti-depressants), toxic or metabolic-endocrine disorders, and changes in electrolytes [17, 49, 50]. However, these factors generally just pose as a temporary problem. The much rarer causes of weaning failure are encephalitis with the involvement of the respiratory centre, or structural damage caused by tumours, elevated cerebral pressure, ischemia, or bleeding $[17,45]$. A central disruption to breathing is accompanied by other severe functional disturbances, often rendering such patients incapable of becoming candidates for weaning.

\subsubsection{Neural Drive}

Despite an intact central respiratory drive, functional disruption can also occur at the load/adaptive capacity level of the peripheral nervous system (PNS) and musculature [51]. Accordingly, diseases associated with the musculature or PNS, such as polyneuropathy, myopathy, or dysfunctional neuromuscular transmission (e.g., myasthenia gravis), can lead to weaning failure.

The most common causes are not only metabolic-toxic lesions such as CIP (see Chapter 4.5), but also primary diseases of either the PNS or neuromuscular transmission, such as spinal muscular atrophy, amyotrophic lateral sclerosis, Guillain-Barré syndrome, or myasthenia gravis [52-54].

Furthermore, neural drive can be adversely affected by direct disruption of the phrenic nerve, or by spinal cord lesions above the level of C4 [55]. However, lesions below C4 can also have an unfavourable influence on the load-capacity ratio by impairing the auxiliary respiratory muscles.

\subsubsection{Respiratory Muscles}

In the context of ICU-acquired weakness (ICUAW) [52], which can develop during the course of intensive care therapy with MV, structural and functional lesions can be induced not only in the nerves, but also in the muscles per se (CIM), especially in cases of septic compli- 
cations [53, 56-59]. This is where kidney failure, a diabetic metabolic state, high doses of corticosteroids, and slow-acting, non-depolarising muscle relaxants (as opposed to the short-acting variety) serve as co-factors, especially when a number of these are present [60-63] (see Chapter 4.5). A second common reason for respiratory pump weakness is an isolated, almost always reversible, inactivation atrophy of the diaphragm muscles, known as ventilator-induced diaphragmatic dysfunction (VIDD) [64-66] (see Chapter 5.3.2). This can arise after just a few days of controlled MV if there are no interposing phases of assisted or spontaneous breathing. In addition, overly long phases of spontaneous or assisted breathing at the beginning of the weaning process can overload the already-atrophied muscles. Additional causes include previously experienced or acute (mostly inflammatory or toxic) muscle diseases such as degenerative myopathy and muscular dystrophy.

With regard to prognosis, ICUAW worsens the patient morbidity rate and, along with the associated healthcare costs, increases the 1-year mortality rate [67].

\subsubsection{Muscle Overloading}

A considerable number of diseases and mechanisms can lead to chronic muscle overloading of the respiratory pump. During the actual weaning phases, it also depends on the current capacity of the musculature itself, in order to tolerate an additional respiratory load (volume or pressure). It is not uncommon for treatment-acquired muscle insufficiency to be combined with the disease that was originally responsible for MV and which has led to harmful continuous loading or overloading of the respiratory pump, such as COPD.

4.2.4.1. Airway Diseases. One of the most common reasons for weaning failure is pressure loading on the respiratory pump, which arises from obstruction of the airways. This is often aggravated by hyperinflation of the lungs, which can lead to a deterioration in efficiency through the adverse mechanical force that develops in association with flattening of the diaphragm; this, in turn, results in high oxygen demand and usage [40,41, $68,69]$. This scenario is well exemplified by COPD. Such a situation can become secondarily aggravated through the use of an artificial airway, namely a tube or tracheal cannula [70], which can lead to an additional increase in respiratory load via an elevated level of resistance. On the other hand, an artificial airway, particularly a tracheostoma, can also serve as a form of respiratory relief for spontaneously breathing patients [71-73].
An increased respiratory load can also arise from the displacement of secretions, inflammatory or tumour-induced stenoses of the airways, and pathological changes to the upper airways following extubation/decannulation [74-77].

4.2.4.2. Thoracic Restriction. Thoracic restriction reduces compliance, with a corresponding increase in the work of breathing. The most common transient causes of thoracic restriction are extensive pleural effusions, as well as fluid accumulation in the thoracic soft tissue due to volume overload. Pronounced thoracic deformities such as scoliosis, or extensive post-tuberculotic pleural fibrosis, can also lead to thoracic restriction [17]. Furthermore, following cardiac and thoracic surgery, an impairment to musculoskeletal integrity, a phrenic nerve lesion, or even pain, may result in an increase in respiratory load and a reduction in capacity, followed by a prolonged weaning process after the intervention [78-81]. This can be aggravated through the loss of lung parenchyma that occurs after lung resection $[82,83]$.

\subsection{Hypoxic Respiratory Failure following Interstitial} Lung Disease and Cardiopulmonary Interactions

The most common cause of hypoxic respiratory failure is represented by a ventilation-perfusion mismatch. Moreover, increased levels of interstitial fluid (such as in ARDS) or fibrotic scarring of the lung parenchyma can reduce lung compliance as well as the surface area for gas exchange; this, in turn, leads to an additional load on the respiratory pump [17]. Similarly, hypercapnic respiratory failure can occur in oxygenation disorders such as atelectasis or inflammatory infiltration. This is due to: (i) an increased need for ventilation in order to maintain adequate oxygenation, and (ii) an elevated work of breathing on the basis of diminished lung compliance. This event is often first unmasked during the spontaneous breathing phases of the weaning process, since during controlled MV, only hypoxic insufficiency remains obvious, whereas the work of breathing is taken over by the ventilator.

Intrathoracic pressure changes during ventilation can influence the pressure gradient not only between the venous system and the right side of the heart, but also between the left ventricle and the systemic circulation [8486]. An increase in intrathoracic pressure (under positive-pressure MV) leads to a rise in right atrial pressure and a simultaneous fall in transmural pressure of the left ventricle. As a consequence, there is less venous backflow to the right side of the heart, while left ventricular output increases. Through the corresponding reductions in pre- 
load and afterload, the intrathoracic blood volume declines [87]. In contrast, a reduction in intrathoracic pressure (e.g., via inspiration during spontaneous breathing with negative pleural pressure) improves venous backflow to the right side of the heart. As a result, however, the transmural pressure gradient in the left ventricle increases, leading to an increased left ventricular afterload. At the same time, the intrathoracic blood volume rises. The haemodynamic effects of spontaneous breathing and MV are essentially dependent not only on cardiac pump function, but also on the current volume status of the patient.

In patients with impaired left ventricular pump function, altered intrathoracic pressure during the start of the spontaneous breathing phase of the weaning process can result in a significant increase in preload and afterload via increases in venous backflow and negative pleural pressure $[87,88]$; this can essentially lead to an acute deterioration in cardiac pump function, with forward and backward heart failure. This especially pertains to spontaneous breathing phases with increased breathing effort and deep excursions, which are associated with a correspondingly high negative inspiratory pleural pressure. In the face of either reduced left ventricular pump function or an increase in left ventricular filling pressure, as seen in HFpEF (heart failure with preserved ejection fraction), the switch from controlled MV to spontaneous breathing can lead to a rise in left atrial pressure, with subsequent pulmonary venous congestion, a decline in gas exchange and a resulting further increase in the work of breathing. Weaning-induced pulmonary edema can then result, further complicating the weaning process [89].

Furthermore, switching abruptly between controlled MV and spontaneous breathing can result in a markedly higher oxygen demand (e.g., of the respiratory muscles), and hence an increased myocardial workload. This situation is particularly critical for patients with coronary heart disease, since myocardial pump function can worsen under these conditions.

The higher metabolic demand for oxygen that is associated with cardiac decompensation is often accompanied by compensatory increases in heart frequency and blood pressure, which, in turn, facilitate weaning failure $[86,90]$. The weaning process can similarly be compromised by pre-existing valvular heart disease (e.g., mitral insufficiency). An elevation in left ventricle afterload with an increase in the end-diastolic filling pressure of the left ventricle can, for example, worsen the extent of mitral insufficiency.

A rise in pulmonary vascular resistance that is potentially aggravated by accompanying hypoxic pulmonary vasoconstriction can trigger an increase in right ventricular afterload and a reduction in cardiac output, thus leading to an imbalance between oxygen transport demand and capacity. In the context of interventricular dependency, a septum shift to the left can result in poorer filling of the left ventricle, a decrease in left ventricular stroke volume, and a subsequent reduction in oxygen supply to the body [91, 92].

These types of cardio-pulmonary interactions $[87,88$, $91,92]$ and interventricular dependence carry particular significance for the weaning process. Therefore, weaning failure is not only facilitated by the effects of pre-existing pulmonary diseases on cardiac function, but also by those of heart disease on ventilation, pulmonary compliance, and respiratory load. Through increases in the perfusion and oxygen demand of the respiratory muscles, the combination of reduced pulmonary compliance caused by heart failure, gas exchange dysfunction, and a secondarily-increased work of breathing promotes a rise in cardiac output volume, with the risk of further cardiac decompensation. Pre-existing atrial fibrillation alone is associated with a longer duration of MV and an increased risk of weaning failure [93]. Stress or septic cardiomyopathy, or even an elevated basal metabolic rate in inflammatoryseptic disorders, can lead to cardiac decompensation, with negative effects on the work of breathing - especially in the case of pre-existing cardiac lesions. This, in turn, can compromise an otherwise stable weaning situation. Therefore, a comprehensive cardiopulmonary differential diagnosis is obligatory, and should include the determination of BNP or NT-pro-BNP levels, echocardiography [94-96] and, if necessary, the use of a pulmonary artery catheter [97-99].

\subsection{Oxygen Transport and Consumption}

The transport of oxygen gas molecules to the mitochondria can be disrupted on many levels. An important point for considering oxygen uptake is the reduction in lung perfusion both in cardiac failure, and in diseases with increased pulmonary arterial pressure or resistance (pulmonary hypertension, pulmonary embolism). When the oxygen-binding curve shifts to the right (such as in acidosis) through an alteration in 2,3 diphosphoglycerate concentration, oxygen release within the capillary bed is actually improved; however, this also effectuates slower oxygen uptake in the lungs, resulting in a compensatory increase in ventilation and an inevitably increased load on the respiratory pump. Furthermore, each increase in total oxygen consumption (such as in fever; permanent infections and especially bronchial infections with im- 
paired cough and bronchial clearance during MV ventilation; pain, tachycardia, or inadequate use of catecholamines) inevitably places an additional burden on the cardiac/respiratory pump. This particularly holds true when oxygen uptake and transport are already impaired due to pre-existing heart failure [87].

Cerebral disturbances associated with an unnecessarily high degree of muscle loading also lead to increases in ventilation and respiratory pump loading, with a higher oxygen demand. Inadequate ventilator settings can also aggravate the situation, not only in the aforementioned case, but in general $[100,101]$ : by increasing dead-space ventilation, they lead to a further disruption in gas exchange. Insufficient trigger settings on the ventilator with subsequent patient-ventilator asynchrony can result in dynamic hyperinflation (such as in COPD), a reduction in pulmonary compliance, and an increased work of breathing, leading to higher oxygen consumption [102].

Besides disruptions of gas transport and exchange in the lung parenchyma, a haemoglobin deficiency (i.e., anaemia) can also serve as a decisive factor in weaning failure. The presence of anaemia leads to a compensatory load on the respiratory and cardiac pumps, which then increases the risk of weaning failure [103]. In patients with a high respiratory pump load, it was demonstrated that the correction of anaemia was associated with a proportional reduction in the work of breathing $[104,105]$ (see Chapter 5.6.1.2).

\subsection{Critical-Illness-associated Polyneuropathy and Critical-Illness-associated Myopathy}

The incidence of CIP and CIM in critically-ill patients ranges between 30 and 70\% [106].

CIP is a predominantly distally-accented, sensorimotor-axonal polyneuropathy [106], and can contribute to weaning failure as well as increases in both the mortality rate and length of the stay in hospital $[107,108,109]$.

The pathophysiology of CIP/CIM is complex, with a number of factors playing a role; these include microcirculatory dysfunction (e.g., within the scope of sepsis), increased expression of selectin and cytokines, increased cell membrane permeability, mitochondrial dysfunction with reduced adenosine triphosphate (ATP) synthesis, and neurotoxic processes (especially those involving metabolic free radicals) [53, 107].

The clinical risk factors that can facilitate the development of CIP/CIM are as follows: systemic inflammatory response syndrome (SIRS), sepsis, kidney failure, multiorgan failure, MV, age, sex, general burden of disease, hypotension, hyperosmolarity, parenteral nutrition, low serum albumin levels, immobilisation, hyperglycaemia, certain medications and hypoxia $[53,107,110,111]$.

In most cases the diagnosis is made clinically, whereas a neurological examination (nerve conduction studies and electromyography) [112], with emphasis on nerve conduction testing of the peroneal and sural nerves, serves as a useful complement [113-115]; a reduction in the amplitude of compound muscle action potentials of the peroneal nerve represents a particularly sensitive diagnostic marker [113, 114].

The difference between CIP and CIM is important in terms of prognosis. Whereas an isolated CIM is completely reversible (in principle), more than $50 \%$ of patients with CIP retain permanent deficits [116]. If CIP/ CIM persists even after discharge from hospital, it is associated with a higher 1-year mortality rate and an increase in health care expenditure [67].

Paediatric patients can also develop CIP or CIM, although the incidence here is potentially lower than that in adult patients [117].

It holds true for all patients that the regression of symptoms can take place over a protracted period of many weeks or months, so that this condition should continue to be monitored even after successful weaning. While the effects of CIP/CIM are easy to identify in the muscles of the extremities, it is more difficult to recognise and evaluate the consequences of these conditions on breathing and swallowing, not only during the weaning process, but also in the phase that follows.

Dysphagia can also develop in the presence of CIP/ CIM during MV/long-term MV [118, 119]. The longterm prognosis for dysphagia in CIP/CIM following ventilation is good, where a complete restoration of the ability to swallow is observed in the majority of patients [119]. Further details on dysphagia, including the associated diagnostic approaches and therapies, can be found in Chapter 5.6.1.5.

Persistent respiratory muscle weakness occurs more often than peripheral muscle weakness following prolonged weaning. Dres et al. [120] reported that $92 \%$ of patients in the "difficult to wean" category had diaphragmatic dysfunction, whereas only $46 \%$ exhibited ICUAW; in the case of prolonged weaning patients, these figures stood at 100 and $50 \%$, respectively. This underscores the importance of assessing, diagnosing, and treating muscle function separately. As in weaning, the goal of the ensuing rehabilitation phase is to improve respiratory muscle competence and minimise load. In this context, the type of MV used during the rehabilitation phase may well change (Chapter 6.4). 


\subsection{Additional Metabolic Aspects}

All organs including the respiratory muscles have a basal metabolic supply, whose impairment through metabolic disorders (hypo/hyperthyroidism, adrenal insufficiency, hyperglycaemia), electrolyte disorders (hyperkalaemia, magnesium and phosphate deficiencies), albumin deficiency, or exogenous endocrine factors such as glucocorticoidsteroid therapy, can lead to functional disorders [17, 45, 121]. Furthermore, metabolic alkalosis can promote weaning failure by attenuating respiratory drive with compensatory hypercapnia $[122,123]$. Similarly, metabolic acidosis should also be considered as a cause of prolonged weaning, since it places a further strain on the already overloaded respiratory pump through ventilatory compensation [124].

The topic of nutrition will be discussed in detail in Chapter 5.6.1.3 (Improving Nutritional Status and Metabolism).

\subsection{Special Characteristics of Paediatric Patients}

Sauthier et al. [125] suggest that prolonged weaning in children is defined as an invasive MV duration of $\geq 21$ days, for at least $6 \mathrm{~h} /$ day. Weaning failure after prolonged MV and a subsequent ventilator dependency that is due to respiratory pump failure is most commonly the result of congenital (neuro)-muscular diseases, or syndromic conditions with muscle hypotonia and a possible thoracic deformity. Congenital or acquired disorders of the respiratory centre, such as central hypoventilation syndrome or cerebral injuries (e.g., following perinatal asphyxiation) [126-129], represent rare causes of weaning failure. Innervation disorders are observed in upper spinal cord injuries as well as congenital or acquired diaphragmatic weakness [130]. Acquired diseases of the respiratory pump that occur through conditions such as CIP, enterovirus-associated paralysis [131], or acquired inactivation of the respiratory muscles, rarely cause weaning failure in children.

Muscle overloading of the healthy respiratory pump more often finds its cause in an obstruction of a child's narrow or unstable airways. Transient swelling of the larynx and tracheal wall due to intubation trauma can lead to post-extubation stridor, which is the cause of extubation failure in up to $35 \%$ of cases $[126,129]$. Long-term intubation can give rise to fibrotic subglottal stenosis; here, weaning is often not possible before a tracheotomy is applied. Moreover, congenital deformities of the airways, whether they be mechanical (e.g., laryngeal stenosis) or functional (tracheomalacia), can lead to weaning failure and require differentiated treatments [132, 133].
Gas exchange disorders that arise from diseases of the lung parenchyma can certainly prolong the weaning process $[126,129,134]$; however, long-term ventilator dependency is rather rare in this situation $[127,128,135]$ and arises mainly after long-term ventilation associated with premature birth (chronic lung disease in premature infants) or ARDS (albeit more rarely) [127, 136]. In children with congenital heart defects, weaning failure has primarily been linked to the duration of ventilation [137], as well as the presence of an additional pulmonary disease [138].

\section{Weaning Strategies}

Invasive long-term MV and prolonged weaning are associated with secondary complications such as volutrauma, barotrauma, atelectrauma and biotrauma, tracheal lesions, the development of ventilator-associated pneumonia, and a significantly increased risk of mortality $[8$, $139-141]$. Only around $41 \%$ of patients with a duration of long-term MV more than 14 days live for one more year following discharge from hospital [142].

Prompt initiation of the weaning process is fundamental in terms of preventing long-term MV and prolonged weaning. According to Cook et al. [143], weaning from MV begins with intubation.

Some of the therapeutic strategies for weaning from MV described in this chapter, such as protocol-based assessment of weaning capacity, management of sedation and delirium, and early mobilisation, are not specific measures in the prolonged weaning process. They rather begin in the acute ventilation phase, provide the foundation for successful weaning, and can help to shorten or even avoid long-term MV and prolonged weaning. The scientific evidence for the effectiveness of these measures usually relates to the "simple" or "difficult" phases of weaning. Currently, there are no high-evidence level scientific evaluations that primarily focus on the effectiveness of these measures in patients undergoing prolonged weaning.

\subsection{Strategies to Prevent Long-Term MV and \\ Prolonged Weaning}

The following sub-chapters will present the results and conclusions from studies on simple and difficult weaning, which are of particular importance in terms of preventing long-term MV and prolonged weaning. 
Table 5. Readiness-to-wean criteria [17]

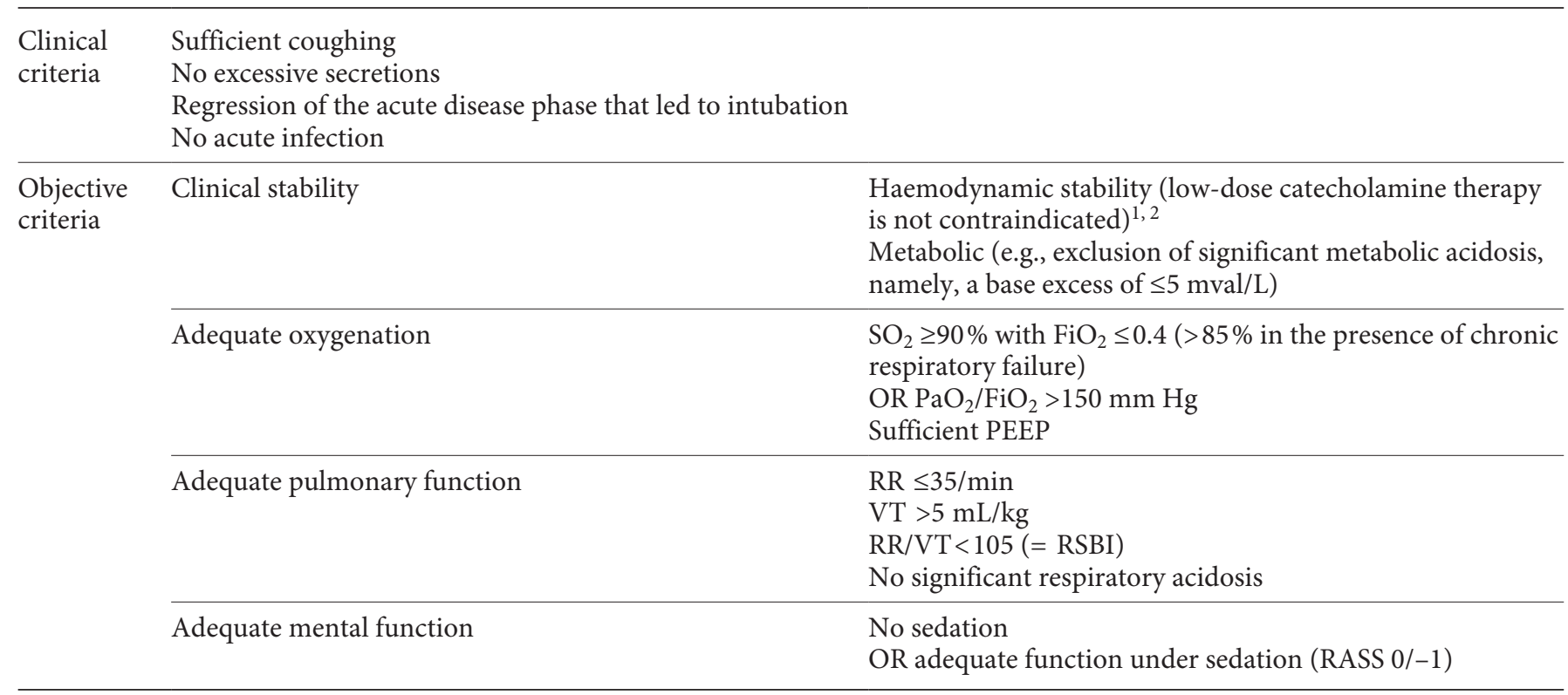

\footnotetext{
${ }^{1}$ See the S3 Guideline "Infarct-related cardiogenic shock: Diagnosis, monitoring and therapy"; Registration number: 019-013 [145]. ${ }^{2}$ Explanation in text. RR, respiratory rate; Vt, tidal volume; PEEP, positive end-expiratory pressure; RASS, Richmond agitationsedation scale; RSBI, rapid shallow breathing index; $\mathrm{FiO}_{2}$, fraction of inspired oxygen; $\mathrm{SO}_{2}$, oxygen saturation (measured by pulse oximetry).
}

\subsubsection{Assessment of Weaning Potential}

Irrespective of the type of ventilation interface used in patients undergoing invasive $\mathrm{MV}$, the basic prerequisite for both reducing ventilation time and successful weaning is to already begin assessing the criteria that can indicate a "readiness to wean" in the acute phase of MV (see Table 5).

It is important to note that weaning predictors such as inspiratory pressure, static compliance or RSBI each do not show any positive or negative value that allows the estimation of weaning ability alone [144]. Therefore, to properly evaluate the weaning potential of patients, it is necessary to consider a number of parameters, as outlined in Table 5. Nonetheless, these parameters only serve as indicators, and can differ in individual cases.

The S3 Guideline "Infarct-related cardiogenic shock: Diagnosis, monitoring and therapy" contains threshold values for heart frequency and blood pressure that have been revised and adapted to specific clinical situations. Therefore, target values for heart frequency and blood pressure have been omitted from Table 5 and should instead be referred to in the aforementioned S3 Guideline [145]. Similarly, if the patient is already receiving cate- cholamine therapy, this may need to be evaluated when reviewing the patient's readiness to wean. Low-dose catecholamine therapy in this setting is considered to be in the range of $0.05-0.1 \mu \mathrm{g} / \mathrm{kg} / \mathrm{min}$.

Figure 2 presents a flow diagram of the evaluation steps taken to determine the patient's extubation capacity. In accordance with the revised S3 Guideline on the management of delirium, analgesia and sedation [146], an adequate assessment of neurological and neurocognitive function as well as the patient's ability to cooperate requires a goal-oriented suspension of sedation (SAT, spontaneous awakening trial) in combination with an SBT; in patients with a RASS (Richmond Agitation Sedation Scale) $\leq-2$, sedation interruption should be performed on a daily basis.

The course of weaning is essentially divided into 2 stages, depending on the type of interface (mask, tube or tracheal cannula). During the first stage, the patient's readiness to wean is assessed under MV on a daily basis. During the second stage, an SBT is performed (see Fig. 3). This diagnostic test plays a decisive role in determining whether extubation will be successful. 


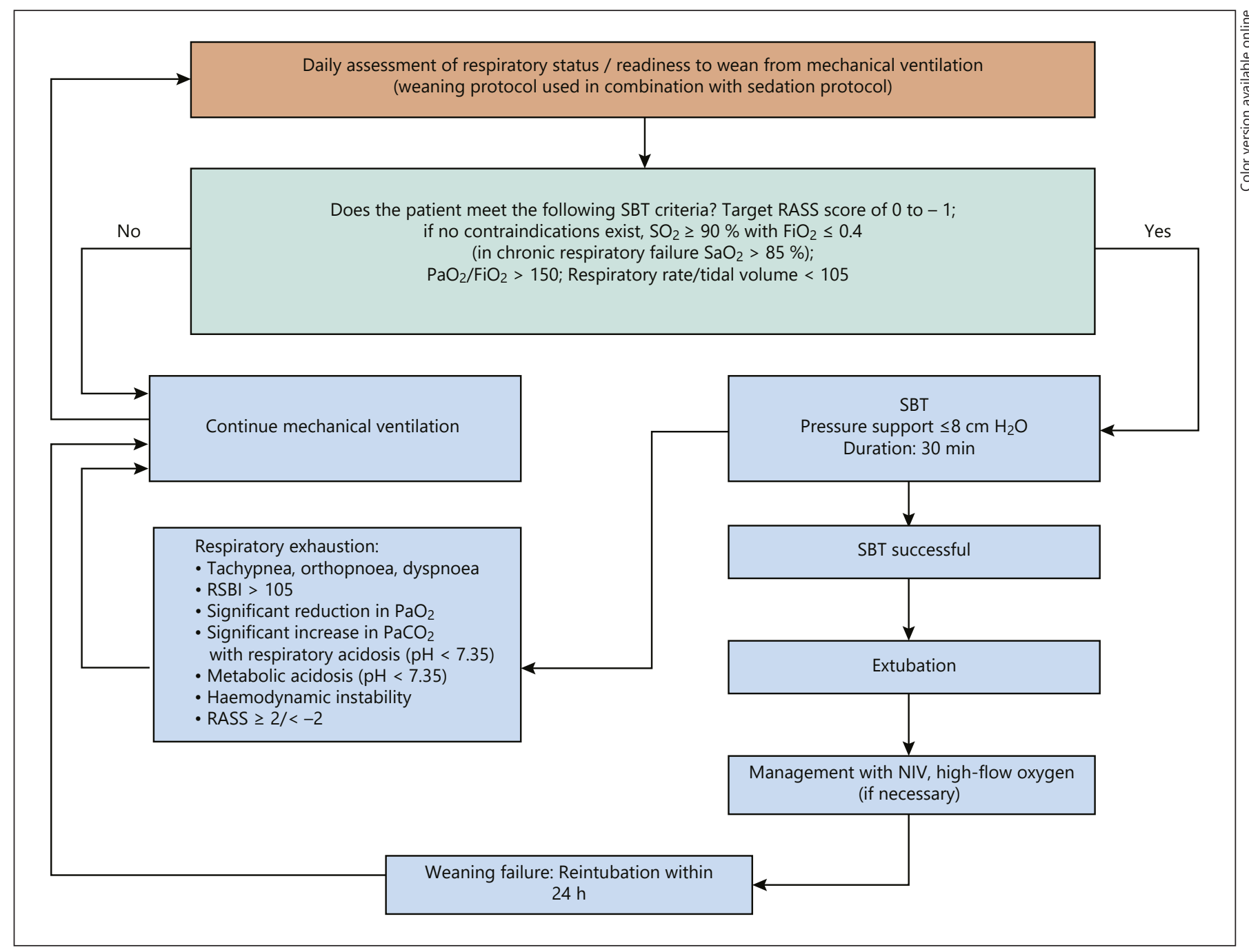

Fig. 2. Flow diagram of the daily routine for screening respiratory status in weaning [147]. $\mathrm{SO}_{2}$, oxygen saturation; $\mathrm{FiO}_{2}$, inspired oxygen fraction; $\mathrm{PaO}_{2}$, partial pressure of arterial oxygen; RASS, Richmond agitation-sedation scale; NIV, non-invasive ventilation; RSBI, rapid shallow breathing index [rerif].

\subsubsection{Management of Sedation, Anxiety, Agitation} and Pain

An important prerequisite for successful extubation or decannulation is an alert and co-operative patient; indeed, over-sedation too often leads to an unnecessary prolongation of MV [148, 149].

In accordance with the recommendations outlined in the current S3 Guideline on the management of delirium, analgesia and sedation [146], the weaning protocol should be used in combination with a sedation protocol (sedation measures in accordance with the S3 Guideline) in invasively-ventilated patients undergoing the weaning process. The S3 Guideline recommends that sedation should generally be reserved for special situations or indications, and needs not be automatically carried out in every invasively ventilated patient. Therefore, sedation should either be avoided, or performed on a targeted-oriented basis. A daily attempt at waking patients with prescribed sedation is reserved for those with a RASS $\leq-2$. Target-oriented sedation is carried out by setting a RASS target of $0 /-1$ and regularly reviewing and documenting the depth of sedation at least every 8 hours.

There are different conventional scoring systems available, including the Ramsay Sedation Scale [150], the Riker Sedation-Agitation Score (SAS) [151] and the RASS (Richmond agitation-sedation scale). The S3-Guideline 


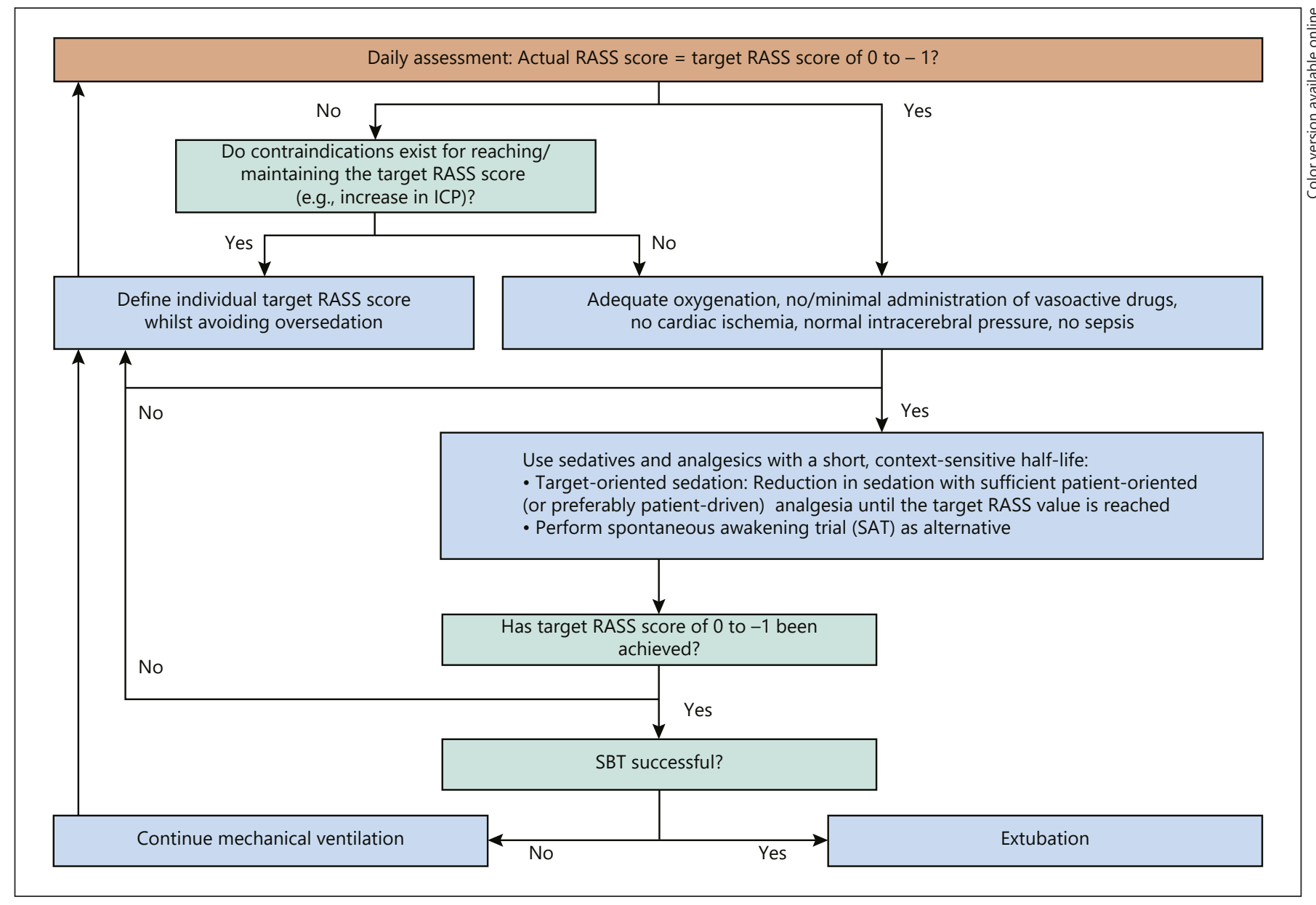

Fig. 3. Flow diagram outlining the management of analgesia and sedation in non-prolonged weaning patients [146, 158]. RASS, Richmond agitation-sedation scale; SAT, spontaneous awakening trial; SBT, spontaneous breathing trial; ICP, intracranial pressure [rerif]).

on the management of delirium, analgesia and sedation [146] recommends monitoring sedation with the aid of the RASS, which is considered to be a valid gold-standard measure in intensive care patients $[152,153]$. Accordingly, preference in weaning should also be given to the use of a valid RASS score (see Fig. 3).

The sedation score is still too rarely used in Germany [154]. Besides the depth of sedation, pain should also be monitored every $8 \mathrm{~h}$. This can be performed not only in awake patients with scales such as the visual analogue scale (VAS) or a numeric/visual rating scale (NRS/VRS), but also in sedated patients with the aid of clinical symptoms (e.g., facial expression, tachycardia, diaphoresis, blood pressure patterns, respiratory rate, lacrimation), or scoring systems such as the behavioural pain scale (BPS) for intubated and non-intubated patients [155]. Delayed awakening, prolonged states of deliria and subsequent complications (e.g. ventilator-associated pneumonia) lead to prolonged MV. Several studies [156-158] have been able to demonstrate that a significant reduction in the duration of MV was possible when a sedation protocol was used during invasive MV. In the studies by Kress et al. [156] and Carson et al. [157], this was achieved by daily interruption of sedation and the so-called awakening phases (spontaneous awakening trial, SAT). The use of a protocol for depth of sedation has also been reported to reduce the duration of MV and the length of stay on the ICU $[156,159]$.

\subsubsection{Daily Sedation Interruption}

When combined with an SBT, interrupting the patient's sedation each morning (as opposed to continuous seda- 
Table 6. Recommendations for sedation from the S3 guideline, "Management of delirium, analgesia and sedation in intensive care medicine" [146]

\begin{tabular}{|c|c|}
\hline $\begin{array}{l}\text { Recommendation number } \\
\text { and level according to the } \\
\text { DAS Guideline }\end{array}$ & Recommendations \\
\hline $\begin{array}{l}\text { 4.5-4.8; Recommendation } \\
\text { Level A: "recommended" }\end{array}$ & $\begin{array}{l}\text { To sufficiently monitor sedation depth and facilitate the early implementation of protocol-based management, it is } \\
\text { recommended that sedation be evaluated by means of a valid measuring scale at least once per shift (i.e., every } 8 \mathrm{~h} \text { ), } \\
\text { and an objective sedation goal is regularly established, which can potentially be adapted to changes in the clinical } \\
\text { situation }\end{array}$ \\
\hline $\begin{array}{l}\text { 5.e.1; Recommendation } \\
\text { Level B: "suggested" }\end{array}$ & $\begin{array}{l}\text { Administration of alpha- } 2 \text { agonists is suggested for reducing stress and autonomic attenuation in adult patients } \\
\text { undergoing intensive care therapy }\end{array}$ \\
\hline $\begin{array}{l}\text { 5.e.3; Recommendation } \\
\text { Level A: "recommended" }\end{array}$ & $\begin{array}{l}\text { Treatment of psychotic symptoms (at the onset of or during delirium, or in isolated occurrences) with neuroleptics } \\
\text { is recommended }\end{array}$ \\
\hline $\begin{array}{l}\text { 5.e.4; Recommendation } \\
\text { Level B: "suggested" }\end{array}$ & $\begin{array}{l}\text { A day-night rhythm should be aimed for in intensive care patients, whereby the use of medicinal and non-medicinal } \\
\text { measures are suggested, e.g., optimisation of the surrounding conditions on the ICU (reduction of light and noise, } \\
\text { and minimisation of necessary measures at night) }\end{array}$ \\
\hline $\begin{array}{l}\text { 5.d.1; Recommendation } \\
\text { Level A: "recommended" }\end{array}$ & $\begin{array}{l}\text { It is recommended that the use of propofol be considered for invasively-ventilated patients (off-label use: after } 7 \\
\text { days of administration, or in patients under } 16 \text { years of age; dose limit }<4 \mathrm{mg} / \mathrm{kg} \text { body weight/hour) }\end{array}$ \\
\hline $\begin{array}{l}\text { 5.d.2; Recommendation } \\
\text { Level O: "can be performed" }\end{array}$ & Inhalative sedation can be utilised in invasively-ventilated patients when aiming for short periods of awakening \\
\hline $\begin{array}{l}\text { 5.d.3; Recommendation } \\
\text { Level O: "can be performed" }\end{array}$ & With adequate monitoring of sedation, midazolam can be used for sedation with a RASS target score of $\leq-2$ \\
\hline
\end{tabular}

tion) in the scheme of a strictly organised sedation strategy, leads to a shorter period of sedation and hence a reduction in the duration of MV and the length of the ICU/hospital stays [158]. Even when more patients carried out self-extubation, this did not influence the rate of reintubation. However, the most important finding of the study was that the strict combination of a sedation protocol with a weaning protocol reduced the 1-year mortality rate.

Through the availability of new evidence, the significance of daily SBTs and waking the patient has had to be re-evaluated. To this end, a systemic review showed that daily interruption of sedation was not superior to protocol-based sedation. More recent studies have shown that a protocol-based sedation regime aimed at keeping patients as awake as possible, or only lightly sedated, has no disadvantages compared to wake-up attempts. These socalled daily sedation interruptions (DSIs) are at best indi- cated for deeply-sedated patients, and cannot replace the awake state per se $[146,160,161]$.

According to the recommendations outlined in the S3 Guideline on the management of delirium, analgesia and sedation [146], either no sedation, or a target-oriented sedation regime with the designation of a target RASS score should also be performed during prolonged weaning. The symptom-oriented treatment of delirium, agitation and pain also follows the recommendations of the S3 Guideline [146]. A flow diagram describing the management of pain and sedation is presented in Figure 3 [146].

\subsubsection{Management of Sedation}

During the prolonged weaning phase, approaches to the monitoring and treatment of anxiety, agitation and pain often need to be tailored to patients on an individual basis. The corresponding recommendations for 
Table 7. Recommendations for the management of delirium from the S3 Guideline, "Management of delirium, analgesia and sedation in intensive care medicine" [146]

Recommendation number Recommendations
and level according to the
DAS Guideline

\begin{tabular}{ll}
\hline $\begin{array}{l}\text { 4.11; Recommendation } \\
\text { Level A: "recommended" }\end{array}$ & $\begin{array}{l}\text { To recognise delirium as a manifestation of organ dysfunction, to remedy the underlying cause and to allow } \\
\text { symptomatic treatment, it is recommended to monitor the patient's delirium with a valid quantitative scale (e.g., the } \\
\text { confusion assessment method for the ICU [CAM-ICU] [164] or the intensive care delirium screening checklist } \\
\text { [ICDSC] [170]) at least once per shift }\end{array}$ \\
\hline
\end{tabular}

2.1; Recommendation
Level B: "not suggested"

2.4; Recommendation Non-pharmacological prevention of delirium is suggested for all adult ICU patients

Level B: "suggested"

2.2; Recommendation Patients with a high risk for developing delirium can receive low-dose haloperidol therapy as prophylaxis

Level O: "can be performed"

5.f.4; Recommendation Continuous administration of alpha-2 agonists is recommended for treating delirium

Level A: "recommended"

5.f.3; Recommendation

Level B "suggested"

Gradual cessation of long-term sedation therapy with the use of adjuvant substances (e.g., alpha-2 agonists) where

5.f.2; Recommendation

Level O: "can be performed" necessary, is suggested for avoiding withdrawal syndromes

Low-dose administration of haloperidol, risperidone, olanzapine or quetiapine can be implemented for the treatment of delirium

this can be found in the 2015 DAS Guideline [146], and are also cited in the same format in the S3 Guideline "Invasive Mechanical Ventilation and Extracorporeal Membrane Oxygenation in Acute Respiratory Insufficiency" [162].

If there is an indication for symptom-oriented treatment of agitation (when the RASS is $0 /-1$ ) the following therapies are recommended: Alpha-2 agonists as a basis therapy for stress reduction; discontinuous application of benzodiazepines for anxiety; neuroleptics for psychotic symptoms and hallucinations; melatonin agonists such as non-pharmacological measures for maintaining a daynight rhythm.

If there is an indication for moderate/deep sedation, the DAS Guideline recommends propofol, while the use of volatile anaesthetics or midazolam is recommended on a "can be used" level (see Table 6).

\subsubsection{Anti-Psychotic and Anxiolytic Treatment Concepts}

5.1.5.1. Pathophysiology of Delirium. Delirium is the most frequent clinical manifestation of brain dysfunction in an intensive care medicine setting. Up to $80 \%$ of ventilation-dependent patients suffer from delirium during treatment on the ICU $[163,164]$. Delirium usually runs a hypoactive course in this setting and therefore often goes unnoticed [165].

Delirium can be distinguished into 2 types: sedationrelated delirium (also known as rapid reversible delirium) and non-sedation-related delirium (also known as persistent delirium). Approximately $20 \%$ of deliria are categorised as the first type and are hence directly induced by sedation [166]. Patients with the 2nd type of delirium have a poorer treatment outcome compared to type $1 \mathrm{pa}-$ tients, since an endogenous functional disorder of the brain likely exists in this type of delirium.

Delirium is associated with a longer period of $\mathrm{MV}$, a longer stay on the ICU, limited cognitive rehabilitation options that carry the risk of persistent deficits, and increased mortality [167-169].

Recent data suggest that the monitoring of delirium alone has a positive effect on the target factors mortality and lengths of ICU/hospital stays. The DAS Guideline [146] explicitly advises against pharmacological prevention, and instead recommends focussing on non-pharmacological measures. However, if needed in high-risk patients, low-dose haloperidol prophylaxis is stated as an open recommendation ("can be done").

5.1.5.2. Treatment of Delirium. The following recommendations are made in the S3 Guideline "Management 
of Delirium, Analgesia and Sedation in Intensive Care Medicine" [146], and are also cited in the same format in the S3 Guideline, "Invasive Mechanical Ventilation and Extracorporeal Membrane Oxygenation in Acute Respiratory Insufficiency" [162]:

Continuous administration of alpha-2 agonists is recommended for symptom-based therapy, as is a tapered sedation regime to avoid withdrawal symptoms. An open recommendation is stated for the low-dose administration of haloperidol, risperidone, olanzapine or quetiapine, taking into account the individual indications (see Table 7).

\subsubsection{Infectiological Aspects of Weaning}

By disrupting gas exchange or increasing bronchial secretions (see Chapter 4.2 and 4.3), pulmonary infections can pathologically lead to higher ventilatory demand or increased respiratory muscle loading, which further impairs respiratory pump function and potentially reduces the chances of weaning being successful. Thereby, evidence from the literature indicates that pneumonia and the presence of multi-resistant pathogens have an impact on weaning outcome $[171,172]$, with a higher rate of weaning failure observed with the detection of tracheobronchial Pseudomonas aeruginosa and Stenotrophomonas maltophilia [171], or MRSA (Methicillin-resistant Staphylococcus aureus), 3-/4-multidrug-resistant Gram-negative bacteria (MDRGN) with ESBL (Extended-spectrum beta-lactamase)-producing enterobacteria, multiresistant Pseudomonas aeruginosa and Acinetobacter baumannii [172]. However, regarding the influence of these pathogens on mortality, there are no consistent findings between the two studies. Even though it is likely that pulmonary and extra-pulmonary infections reflect the severity of the underlying disease, tracheobronchial pathogens (especially multi-resistant ones) must receive special attention in relation to weaning outcome (also see Chapter 5.2.1).

To avoid prolonged weaning, including the weaning process itself, the preparation of a standard approach is recommended to facilitate the early detection and etiological diagnosis of pulmonary and extrapulmonary infections, as well as to enable targeted anti-microbial therapy [173]. This comprises the establishment of strict hygiene measures to prevent pathogenic transfer, which should be regularly assessed for their effectivity. The indication for antibiotic therapy (such as azithromycin, or inhalative antibiotics for Pseudomonas aeruginosa infections) should be determined on an individual basis, since evidence for this is lacking.

Prolonged Weaning: S2k Guideline by the German Respiratory Society

\subsubsection{Spontaneous Breathing Trial (SBT)}

The SBT measures the spontaneous breathing capacity of a patient, and is used as predictive test to determine whether a patient can be extubated. The SBT is performed after ascertaining the patient's readiness to wean, and should be carried out in conjunction with a sedation protocol that adheres to the S3 Guideline recommendations [146] (see Section 5.1.3).

The first 30 minutes of the SBT are decisive in terms of success vs. failure $[174,175]$. The predictive value for a successful intubation is the same for a 30 -min test as it is for a 120 -min test $[174,176]$. As evidenced by multiple large studies [7, 23, 174, 177, 178], the positive predictive value for a successful extubation following a successful SBT is over $80 \%$, providing no other contraindications exist for a successful extubation. To this end, neurological status [179], a pronounced increase in bronchial secretions, and airway obstruction [177] can present as reasons for not carrying out an extubation, despite a successful SBT.

The SBT-based criteria for an extubation cannot be applied at any time in every patient undergoing prolonged weaning. Nevertheless, even in patients with limited vigilance and cognition a SBT can be performed if the individual clinical situation allows. In this context, the difference in spontaneous breathing phases between simple and prolonged weaning should be mentioned. In simple weaning, the SBT is a diagnostic test for evaluating the patient's readiness to be extubated. In prolonged weaning, however, the SBT can aid in the decision-making process for diagnosing the cause of prolonged weaning, and may also be useful for starting intermittent weaning at an early timepoint. In alternation with MV phases, the spontaneous breathing phases (which may last several hours, if applicable) thereby serve to recondition the respiratory muscles $[180,181]$ (see Chapter 5.3.8).

Numerous studies have investigated the methodology for performing an SBT: When the T-piece trial was compared to low pressure support ventilation (PSV, $7 \mathrm{~cm}$ $\mathrm{H}_{2} \mathrm{O}$ [8]; $8 \mathrm{~cm} \mathrm{H}_{2} \mathrm{O}$ [182] or CPAP [183]), there was no difference between these methods in terms of a favourable SBT outcome and successful extubation post-SBT. Some patients experience a problem with respiratory burden, which is caused by the tube during the SBT; this, however, can be offset by applying automatic tube compensation (ATC). In two simple-weaning studies, ATC was compared with either PSV, or PSV and T-piece ventilation $[184,185]$, and no difference found between the methodologies investigated. 
The following parameters suffice for evaluation of the SBT: (i) respiratory rate and (ii) the ratio of respiratory rate to tidal volume (RSBI [175]), which should be measured at the conclusion of the SBT. In this instance, the respiratory rate and RSBI should not exceed $35 / \mathrm{min}$ and $105 / \mathrm{min} / \mathrm{L}$, respectively. If the patient fails the SBT, MV is continued. The cause of SBT failure should be analysed in order to recognise and rectify or eliminate the problem (see Table 4). The MV strategies that can be applied after SBT failure are described in Chapter 5.3. A renewed SBT should preferably be carried out daily, in order not to miss the chance for a potential early extubation.

Recommendation ( $R$ )1: It is recommended to perform an SBT as soon as the patient exhibits readiness to wean.

$R 2$ : Measurement of the rapid shallow breathing index at the end of the SBT is suggested for the evaluation of respiratory muscle fatigue.

\subsubsection{Ultrasound Assessment of the Diaphragm}

Kim et al. used ultrasound to investigate diaphragm function in 88 patients [186]. Diaphragmatic dysfunction was defined either as a vertical deviation of the muscle of $<10 \mathrm{~mm}$, or as paradoxical movement. Twenty-four patients (29\%) exhibited diaphragmatic dysfunction and when compared to patients with proper function, showed a significantly longer weaning time (17 vs. 4 days, $p<$ 0.01 ) and total MV duration ( 24 vs. 9 days, $p<0.01$ ). Further studies are needed to determine the value of this promising method as a weaning predictor. Di Nino et al. investigated diaphragmatic thickening in 63 ventilated patients to determine whether this measure could be used as a predictor of extubation [187]. The percentage change in diaphragm thickness $(\Delta$ tdi $\%)$ during inspiration and expiration in the zone of apposition was documented during assisted and non-assisted spontaneous breathing. The sensitivity and specificity for a $\Delta$ tdi $\%$ of $\geq 30 \%$ was 88 and $71 \%$, respectively. The positive and negative predictive values were 91 and $63 \%$. The area under the ROC curve was 0.79 . In comparison to the $\Delta$ tdi $\% \geq 30 \%$, the RSBI measured during the SBT in 26 patients without ventilatory support did not perform as well (sensitivity, 87\%; specificity, 33\%; PPV, 91\%; NPV, 25\%). Using sonography, Soummer et al. calculated lung aeration in 100 patients at the conclusion of a 60-min SBT and 4 hours after extubation [188]. Patients with post-extubation distress (Group 2) had a significantly higher ultrasound score than those who were definitively weaned (Group 1). The increased ultrasound score observed at the conclusion of the SBT was also significantly higher in Group 2 patients ( 19 vs. $10, p<0.001$; ROC, 0.86 ).
Despite the overall promising collection of data on the use of ultrasound during weaning, this field of research is marked by methodological heterogeneity, which limits the comparability of study findings. The authors of the current guideline suggest that ultrasound will play an important future role not only in the prediction of weaning success, but also in the monitoring of the actual weaning process. However, at the time of completing this guideline revision, it was not possible to derive any clear recommendation from the available data pool (see Chapter 5.3.7).

\subsubsection{Weaning Protocol}

The use of standardised protocols while weaning the patient from MV can aid the overall weaning process. These protocols have been especially developed for simple weaning, where an extubation is planned following a short period of MV. One of the most important factors here is the identification of the earliest time point at which the weaning process should begin. A number of studies support this notion through the observation that approximately $70-80 \%$ of patients who receive MV for more than $24 \mathrm{~h}$ can successfully be weaned from ventilation on the first attempt [17]. This implies that many of these patients could have been weaned even earlier, an assumption that is supported by the observation that only around $50 \%$ of patients need to resume MV after an unplanned self-extubation [189].

The use of a protocol for early weaning leads to a reduction in the total duration of MV, the duration of weaning, and the length of stay on the ICU. Blackwood and colleagues were able to demonstrate this in their 2011 systematic review [190], as well as in their updated version published in 2014 [191]. However, neither of these reviews found an association between the use of a weaning protocol and mortality rate.

Numerous studies have also been able to demonstrate that besides reducing the duration of $\mathrm{MV}$, the use of a standardised weaning protocol can also lead to an increase in the amount of successfully weaned patients $[192,193]$. Furthermore, it has been shown that in patients who were clinically deemed not ready to wean, the transfer to a clinical centre in which a weaning protocol was followed led to the immediate successful weaning from MV in one third of these patients [194]. It should be noted, however, that a weaning protocol does not serve any advantage when used in a well-organised centre with structured ward rounds and maximal staff numbers [195]. Through the use of a weaning protocol in trauma patients, a reduction in ventilator-related complications 
such as nosocomial pneumonia was observed, which was associated with a decrease in mortality rate [196]. An important component of weaning protocols is the patient's state of wakefulness when he/she is supposed to undergo an SBT. This is where a balance between the sedation and the weaning protocol is required. To this end, one study showed that a strictly organised sedation strategy, with daily attempts at waking the patient prior to beginning the weaning process, led to a reduction in the duration of sedation and hence in the duration of MV, as well as shorter stays both on the ICU, and in the hospital in general [158]. The most important finding of the study was, however, that the strict combination of a sedation protocol and a weaning protocol was associated with a decrease in the 1-year mortality rate [158]. Even though the exact mechanisms underlying this difference in mortality rate remain under discussion, a clear recommendation for undertaking this approach can be derived from these findings [193]. These data also serve as a striking example of how different organisational structures can actually influence medical findings.

Based on these data, the S3 Guideline "Invasive Mechanical Ventilation and Extracorporeal Membrane Oxygenation in Acute Respiratory Insufficiency" recommends using a weaning protocol in adult patients who have been invasively ventilated for more that $24 \mathrm{~h}$ [162]; this facilitates the standardised evaluation of the patient's readiness to wean, the undertaking of SBTs and the assessment of criteria for ending invasive $\mathrm{MV} /$ carrying out extubation or decannulation.

Amongst the prolonged weaning studies that have been published, none was found that explicitly investigated the effect of weaning protocols on the duration of MV and weaning, as well as weaning success. In line with both the S3 guideline on Delirium, Agitation and Pain [146] and the S3 Guideline "Invasive Mechanical Ventilation and Extracorporeal Membrane Oxygenation in Acute Respiratory Insufficiency" [162], the level of scientific evidence for the combined use of relevant sedation protocols and weaning protocols is high for patients in Weaning Categories 1 and 2.

The Guideline committee believes that conferring some of the available scientific evidence onto prolongedweaning patients is justified. To this end, the committee provides the following recommendation for the use of protocols in prolonged weaning:

R3: In analogy with patients in Weaning Category 1 or 2 (simple and difficult weaning), the protocol-based standardisation of both the weaning process and analgosedation is recommended for prolonged weaning patients. In addition, the use of simple-to-follow protocols is suggested for the management of agitation, delirium and pain, as well as for the assessment of spontaneous breathing capacity.

\subsubsection{Cuff-Leak Test}

Once ventilated patients have successfully completed an SBT, a complication known as post-extubation stridor can occur after extubation, especially in patients who have been intubated for more than 36 hours. To evaluate the probability of this complication, which has an occurrence rate of between 2 and 16\% [197], a cuff-leak test (measurement of air leakage after unblocking endotracheal tube) can be performed prior to the planned extubation [197, 198]. This allows an estimation of the degree of obstruction expected to occur after extubation takes place [197-199]. The test begins by ascertaining the expiratory tidal volume $\left(\mathrm{V}_{\mathrm{t}}\right)$ with an inflated cuff under the assistcontrol ventilation mode. The measurement is then repeated with a deflated cuff, whereby a markedly lower expiratory volume is expected due to leakage. Prior to the test, endotracheal and oral suctioning should be performed, and following a control check and documentation of the cuff pressure, it should be verified whether the inspiratory and expiratory tidal volumes are equal [200]. Thereafter, the cuff is deflated and the inspiratory and expiratory tidal volumes are monitored over 6 respiratory cycles, whereby the difference in $\mathrm{V}_{\mathrm{t}}$ should become stable. The average of the 3 lowest expiratory tidal volumes is then correlated with the inspiratory tidal volume measured before the cuff was deflated.

A minimal difference of $<130 \mathrm{~mL}$ between both volumes (cuff-leak volumes) is judged as "positive" (i.e. pathological) and can identify patients with post-extubation stridor [199]. However, it should be noted that the cut-off value varies between the different examinations. Two studies defined the limit as $<24 \%$ of the $V_{t}$, three studies as $<110 \mathrm{~mL}$, and one as $<25 \%$ of the $\mathrm{V}_{t}$ [201]. A meta-analysis of nine studies revealed that the cuff-leak test had a mean sensitivity of 0.63 and specificity of 0.86 [202]. Patients with obesity hypoventilation syndrome (OHS) and sleep-related respiratory dysfunction exhibit dynamic obstruction of the upper airways, depending on their stage of sleep and body position. Therefore, the cuffleak test in this group of patients can especially yield a false-negative result. Lemyze et al. postulated that the anatomical relevance of a positive cuff-leak test has not been sufficiently investigated and can yield false-positive results [203], such as in cases where a large-diameter endotracheal tube is combined with a narrow glottis. The au- 
thors carried out fibreoptic laryngoscopy examinations in 34 patients with a positive cuff-leak test, and found that a subset of patients had relatively inconspicuous vocal cords, whereas swelling was observed in the vicinity where the stomach tube and endotracheal tube had compressed the tissue in between (posterior larynx in the area of the posterior commissure); this however, did not present as an impedance to extubation. The use of complementary laryngeal ultrasound in the future may help to better predict post-extubation failure [204].

Based on an analysis of 14 observational studies, the 2017 Clinical Practice Guideline by the American Thoracic Society/American College of Chest Physicians recommends performing the cuff-leak test in patients who fulfil the extubation criteria, and in whom there is a high risk of post-extubation stridor [205]. However, this recommendation carries a high level of uncertainty, given the evidence available. This means that statements about its relevance cannot be made. In particular, the risk of unnecessarily prolonging the duration of MV in the event of a false positive test has led to the test being restricted to high-risk patients (traumatic intubation, MV duration of $>6$ days, endotracheal tube, female gender, reintubation after unplanned extubation); this is due to the fact that the majority of patients who do not receive a cuff-leak test are nevertheless successfully extubated. The vast majority of patients undergoing prolonged weaning fulfil these risk criteria.

In high-risk patients who have a pathological cuff-leak test result but otherwise fulfil the extubation criteria, the guideline by the American Thoracic Society/American College of Chest Physicians [206] recommends the systemic administration of steroids at least 4 hours before the extubation is to take place. This recommendation is based on a meta-analysis of 7 randomised controlled trials (RCTs), where it was shown to have a positive effect on high-risk patients with reduced cuff-leak volumes [207]. Another meta-analysis which was published in 2017 and included 11 RCTs confirmed these findings by showing that the administration of steroids in high-risk patients is associated with a significant reduction in postextubation airway events and reintubation (airway events: risk ratio [RR], 0.34; 95\% CI 0.24-0.48; reintubation: RR, 0.35; 95\% CI 0.20-0.64) [201]. The Number Needed to Treat was 5 for airway complications and 16 for reintubation. Furthermore, an analysis of dosage (cumulative hydrocortisone-equivalent dose between 100 and 1,000 $\mathrm{mg})$, treatment intervals, and treatment frequency (1-4 doses within $24 \mathrm{~h}$, with the final or single dose given 30 min $-4 \mathrm{~h}$ before extubation) was performed, showing the lack of effects for dosage and treatment interval.
In cases where extubation is not possible, the test should be repeated after $12 \mathrm{~h}$.

The value of cuff-leak testing in prolonged weaning has not yet been investigated. In prolonged-weaning patients with a corresponding risk profile for post-extubation stridor, it appears medically reasonable to carry out a cuff-leak test prior to extubation in these patients.

R4: Performing a cuff-leak test prior to extubation is recommended for determining the occurrence probability of post-extubation stridor.

R5: An adjuvant fibreoptic laryngeal examination is suggested for patients with a positive cuff-leak test result, to find the distinct cause of the positive result.

R6: The administration of corticosteroids (at least 4 hours before extubation) is suggested for patients with a pathological cuff-leak test result.

\subsubsection{Early Mobilisation}

The term mobilisation encompasses the measures for implementing passive and/or active functional movement exercises in patients, with the goal of promoting or maintaining functional mobility and hence functional independence. Early mobilisation describes the early implementation of mobilisation measures in critically-ill as well as ventilated patients, which is usually within $72 \mathrm{~h}$ of admission to the ICU [208]. In contrast, positioning describes the changing of the patient's body position in bed, and serves to influence gravity-related effects [208].

Early mobilisation promotes faster rehabilitation in ICU patients, with the goal of maintaining or promoting functional mobility. It also serves to counteract (i) rapid fatigue and atrophy of the respiratory muscle pump and skeletal muscles, (ii) the development of psycho-cognitive deficits, and (iii) the formation of positioning-related skin and soft-tissue injuries [209].

Before movement can occur in the sitting or standing position, the patient needs to be prepared for this process through passive/assisted or active exercises, the acquisition of muscle function and stretching exercises. Passive mobilisation (e.g., special chair) or sitting on the edge of the bed to perform balance training is then undertaken to the point where active mobilisation, standing, walking exercises, and possibly balance and coordination exercises are possible.

The initiation of early mobilisation precedes the prolonged weaning phase, and is understood to be an adjunctive measure in ICU patient therapy for preventing or shortening long-term MV and prolonged weaning.

The available randomised controlled studies on the impact of early mobilisation during the acute phase of 


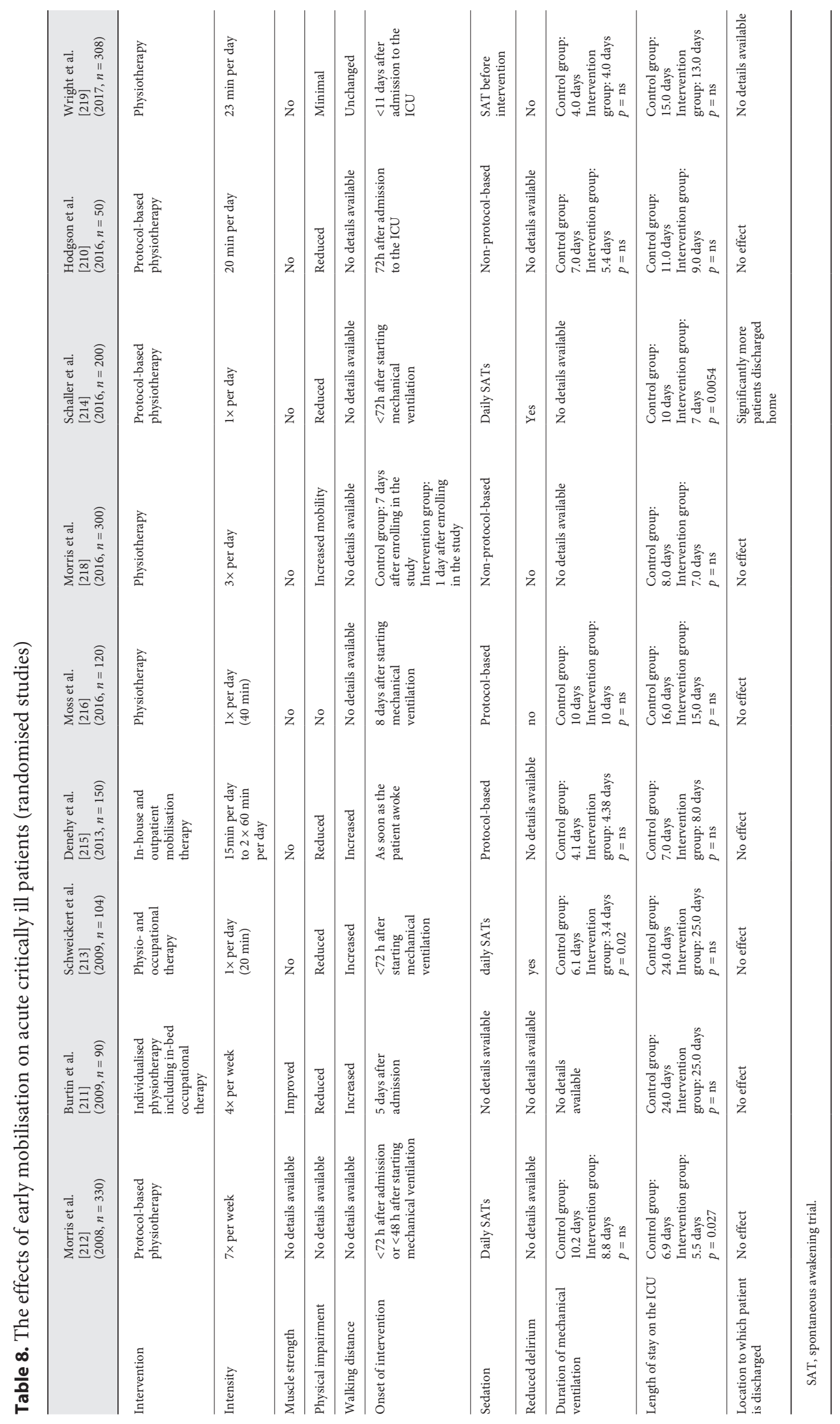


Table 9. Barriers for the implementation of early mobilisation on the intensive care ward [228]

\begin{tabular}{ll}
\hline $\begin{array}{l}\text { Patient-related } \\
\text { issues }\end{array}$ & $\begin{array}{l}\text { Patient is not resilient enough } \\
\text { Haemodynamic instability } \\
\text { Obesity } \\
\text { Pain } \\
\text { Delirium }\end{array}$ \\
\hline Structural issues & $\begin{array}{l}\text { Minimal staff available } \\
\text { No mobilisation program in place }\end{array}$ \\
\hline Process-related & $\begin{array}{l}\text { Lack of planning/coordination } \\
\text { issues }\end{array}$ \\
\hline Cultural issues of daily feasibility screening \\
& $\begin{array}{l}\text { Early mobilisation is not considered to be } \\
\text { important } \\
\text { Lack of a multi-professional therapeutic } \\
\text { approach }\end{array}$ \\
\hline
\end{tabular}

critical illness yield a heterogeneous picture in terms of the acquisition of mobility and muscle function and the duration of MV [217]. Table 8 summarises the published randomised controlled studies in the context of their most important findings.

The value of neuromuscular electrostimulation for preventively effective muscle activation during the acute phase of critical illness has only been investigated in a few studies, including pilot studies [220-224]. These studies reported on the positive effects of electrical muscle stimulation on muscle strength, muscle function, and the maintenance of muscle mass.

Nydahl et al. [225] reported in their study of 116 German ICUs that the implementation of early mobilisation in daily clinical practice is still considerably limited, despite its broad indication. This study found that only $8 \%$ of endotracheally-intubated patients were mobilised outof-bed, while $15 \%$ were not mobilised at all. Similar findings were published in an American study, where $32 \%$ of the patient cohort investigated had received physiotherapy, and a positive association was found between occupational therapy and the absence of MV [226]. Another study carried out in the US showed that in a sub-group of patients who had undergone prolonged weaning for $>14$ days, physiotherapy was prescribed only in $45 \%$ of these patients, most of whom had a tracheotomy, a limited need for sedation or impaired mobility prior to their hospital stay [227]. The intensity level of the physiotherapy was generally described as low, and rarely went beyond passive or active movement of the extremities.

An analysis of the possible hurdles faced in the early implementation of mobilisation on the ICU revealed multiple factors including patient-related, structural and cultural, or process-related issues [228] (see Table 9).

In summary, numerous studies have demonstrated that early mobilisation has a high level of safety and is generally feasible. Since the literature does not define any exclusion criteria for early mobilisation, mobilisation needs to be adapted to acute situations such as untreated fractures, respiratory instability and palliative care patients. The average rate of undesired side-effects occurring from mobilisation treatment is $<1 \%$. These incidences were limited to exceptional cases of oxygen saturation or cardiac instability, and were able to be treated by ending mobilisation therapy.

The process of early mobilisation is facilitated by a multi-professional approach, where roles and responsibilities as well as the timing, intensity and duration of the therapy can be most easily arranged [229, 230].

Structural problems can also impair the comprehensive implementation of regular physiotherapy on intensive care and weaning units [231]:

- The establishment of early mobilisation is an important procedure in terms of documenting complex intensive care treatment, according to which a distinction is made between simple and complex intensive care medicine. The additional work required for physiotherapy services in prolonged weaning are not adequately reflected by the Operation and Procedure Code (OPS) catalogue. Therefore, the corresponding additional costs associated with physiotherapy in prolonged weaning are not represented. This then prevents adequate staffing of physiotherapists in the prolonged weaning sector.

- Given that many ICUs lack permanent physiotherapy personnel that are assigned to the unit on a long-term basis, continuous physiotherapy treatment for patients becomes difficult, particularly for those undergoing prolonged weaning.

- Finally, the various professional bodies offer differing opinions about the role of physiotherapists on the ICU. From our perspective, secretion management, including endotracheal suctioning after mobilisation of the secretions, is an invasive measure that most logically belongs to the tasks of the nursing staff and the physiotherapists. However, physiotherapists still require permission for this following training and delegation by the responsible physicians. Furthermore, speech and respiratory therapists can also be included in the treatment process. 


\subsection{The Significance of Physiotherapy and}

Measurement of Peak Expiratory Flow in Prolonged

Weaning

While early mobilisation on the ICU is considered to be a measure for preventing long-term MV and prolonged weaning, intensive physiotherapy, speech therapy, and occupational therapy all play an important role in the integral rehabilitation concept in prolonged weaning. Similar to the meaning of the term "early mobilisation" during the acute phase of critical illness, the term "mobilisation" is used to describe the passive and/or active movement exercises undertaken during prolonged weaning.

\subsubsection{Physiotherapy in Prolonged Weaning}

There is little to no scientific evidence regarding the allocated numbers of physiotherapists or other therapeutic professionals on intensive care and weaning units [232].

The weaning guidelines by the German Neurorehabilitation Society (Deutsche Gesellschaft für Neurorehabilitation, DGNR) provide an overview of the impact of therapeutic/rehabilitative interventions [233]. Despite the lack of scientific evidence, experts assign a high level of importance to this kind of intervention (particularly physiotherapy) in the prolonged weaning setting. As the main components of treatment, mobilisation and secretion management determine the success or failure of: (i) weaning the patient from the ventilator, (ii) decannulation and (iii) the transfer to a rehabilitation facility for continued therapy. During the complex weaning process, physiotherapy is essential for reconditioning, mobilisation, and secretion elimination, and must be carried out daily at an intensive level (see Quality Indicator [QI] 1). Due to the lack of conclusive studies on this special topic, the recommendations stated below are based on the level of evidence derived from expert opinion.

A study on integrative rehabilitation concepts reported that neuromuscular electrical stimulation partly prevented further muscle atrophy in patients with advancedstage disease who are unable to perform voluntary muscle contraction (e.g., in those with spinal cord injury or pronounced CIP and/or CIM) [234]. Moreover, when electrostimulation was combined with active physiotherapy, the time taken until mobilisation in a wheelchair was achieved was considerably shortened [234]. A metaanalysis of this topic that included nine studies (albeit of a non-homogenous quality) found clear evidence that neuromuscular electrical stimulation was associated

Prolonged Weaning: S2k Guideline by the German Respiratory Society with a growth in muscle mass, volume, and strength, as well as a reduction in MV and weaning duration [235]. An earlier study also observed an effect on muscle proteins, which showed increased breakdown in association with intermittent electrical muscle stimulation [236]. A similar appraisal emerged from a 2016 Cochrane analysis, which showed a significant increase in the strength of the quadriceps femoris muscle, along with an increase in thigh muscle mass [237]. In a study of 16 patients with invasive MV for more that 48 hours, the combination of neuromuscular stimulation (especially of the thigh musculature) with passive movement via a bed pedal exerciser gave rise to a trend towards an earlier and quicker ability to stand and walk, accompanied by a significantly shortened duration of delirium [238]. By means of special training equipment and adaptation to the patient's own performance ability, not only are active movement exercises possible, even when lying in bed, but also passive and active aerobic training of the arm and leg muscles.

Many neurorehabilitation patients, including those undergoing prolonged weaning during the early phases of neurological/neurosurgical rehabilitation, show evidence of infection with multi-resistant pathogens. This leads to reduced contact with medical and nursing staff, and possibly has an influence on the frequency of exercise sessions with the physiotherapist. Despite undergoing rehabilitative therapy, the outcome for these patients is worse than that of those without the colonisation of multi-resistant pathogens, where they show a longer duration of treatment, lower Barthel Index (BI) at hospital admission and discharge, lower BI during early rehabilitation, and low coma recovery scale (CRS) at hospital discharge [239] (see Chapter 5.1.6).

The prerequisite for an integrative rehabilitation concept in prolonged weaning is that both the cardiac and respiratory reserves of the patients are observed in accordance with the recommendations of the European Respiratory Society and the European Society of Intensive Care Medicine Task Force on Physiotherapy for Critically-Ill Patients [240]. This particularly pertains to patients undergoing prolonged weaning, to ensure they are not endangered through exposure to a non-physiologically high load. By observing the limits of these cardiac and respiratory loads, physiotherapeutic treatment can be safely carried out. This is exemplified by a prospective observational study of 1110 patients who each underwent an average of 5-6 physiotherapy sessions: out of this total number of patients, only $36(0.6 \%)$ therapy sessions had to be prematurely stopped, mainly due to cardiac ar- 
rhythmia or hyper-/hypotensive circulatory dysfunction [241]. Mobilisation measures have already been empirically proven to be effective in the early stages of neurological/neurosurgical rehabilitation of patients with $\mathrm{MV}$ [233].

R7: Daily mobilisation of patients undergoing prolonged weaning is recommended, provided there are no exclusion criteria present.

R8: Performing 20 min of mobilisation per day by means of a tiered procedure (beginning with passive mobilisation) based on an in-hospital algorithm is recommended.

QI1: Daily mobilisation: Number of patients mobilised/Number of patients undergoing prolonged weaning.

\subsubsection{Measurement of Peak Expiratory Flow in}

Patients with an Endotracheal Tube or Tracheal

Cannula

Besides obtaining a positive SBT result, the ability to clear secretions by coughing following extubation is crucial for successful weaning in patients with neuromuscular diseases. The measurement of peak cough flow (PCF) is predictive for the risk of obstruction caused by secretions in the lower airways following extubation or decannulation (i.e. in spontaneously-breathing patients). In ALS (Amyotrophic Lateral Sclerosis) patients, a PCF of $\leq 160 \mathrm{~L} / \mathrm{min}$ correlates with outcome [242], which means that weak coughing is associated with a poor prognosis.

Since PCF is markedly lower in intubated and tracheotomised patients (lack of glottis function for generating intrathoracic pressure, tube/cannula-related hinderance of effective coughing), the measurement of peak expiratory flow (PEF) using an in-line peak flow meter on the tube is analogous to PCF during spontaneous breathing $[243,244]$. Values below $60 \mathrm{~L} / \mathrm{min}$ [243] or $35 \mathrm{~L} / \mathrm{min}$ [244] have been identified as predictors of extubation failure. The semi-quantitative analysis of cough strength (semi-quantitative cough strength score, SCSS), for which a score between 0 and 5 is assigned $(0=$ no cough on command, $1=$ audible movement of air through the tube, but no audible cough, 2 = weakly (barely) audible cough, $3=$ clearly audible cough, $4=$ stronger cough, $5=$ multiple sequential strong coughs), was shown in a 2015 study to have a similar diagnostic value to that of the PCF at a cutoff value of 3 [245].

By undergoing corresponding intensive secretion management therapy with a physiotherapist in the postextubation phase, neuromuscular patients with a low PEF can also be successfully extubated and then likely switched to NIV therapy.
R9: The measurement of peak expiratory flow is recommended prior to extubation/decannulation, especially in patients with neuromuscular impairment. An intensive, non-invasive secretion management program is strongly recommended for patients with values below 60 $\mathrm{L} / \mathrm{min}$ after extubation or decannulation.

\subsubsection{Combining Different Procedures}

In addition to adequately resolving respiratory failure, the successful completion of invasive MV requires a sufficient cough strength and swallowing reflex, along with airway patency and secretion volume (no excessive secretion). Quantitative documentation of the various clinical criteria (see Table 5) is therefore useful. In their prospective observational study of 125 burn patients, Smailes et al. showed that combined measurements of endotracheal suctioning frequency and cough peak flow had a strong predictive capacity [246]. A study of 403 patients with extubation failure by Lai et al. identified the cuff-leak test, RSBI, and maximal expiratory pressure as independent risk factors [247].

\subsection{Types of Ventilation Used in Weaning}

5.3.1. Introduction

In the field of modern MV medicine, a multitude of modalities are available, some of which are implemented in prolonged weaning procedures. Controlled and assisted ventilation modes can essentially be distinguished, although there is no strict intersection between these two modes. Despite the huge number of ventilation modes in existence, only a limited number have been established in daily clinical practice. Since very few studies have investigated the role of special ventilation procedures in prolonged weaning, recommendations for selecting a specific ventilation method can only be given with caution. Table 10 provides an overview of the most common modes of MV.

During spontaneous breathing, the work of breathing is accomplished by the respiratory muscles (diaphragm and auxiliary respiratory muscles), whereas during assisted MV, part of the work is taken over by the ventilator. In controlled MV, the work of breathing is exclusively accomplished by the ventilator and the patient remains passive.

Adaptive MV represents a separate group, because the degree of support here varies, depending on either the patient's respiratory drive or the work of breathing.

Stable spontaneous breathing or adequate respiratory drive is generally required for assisted vs. adaptive ventilation modes.
Schönhofer et al. 


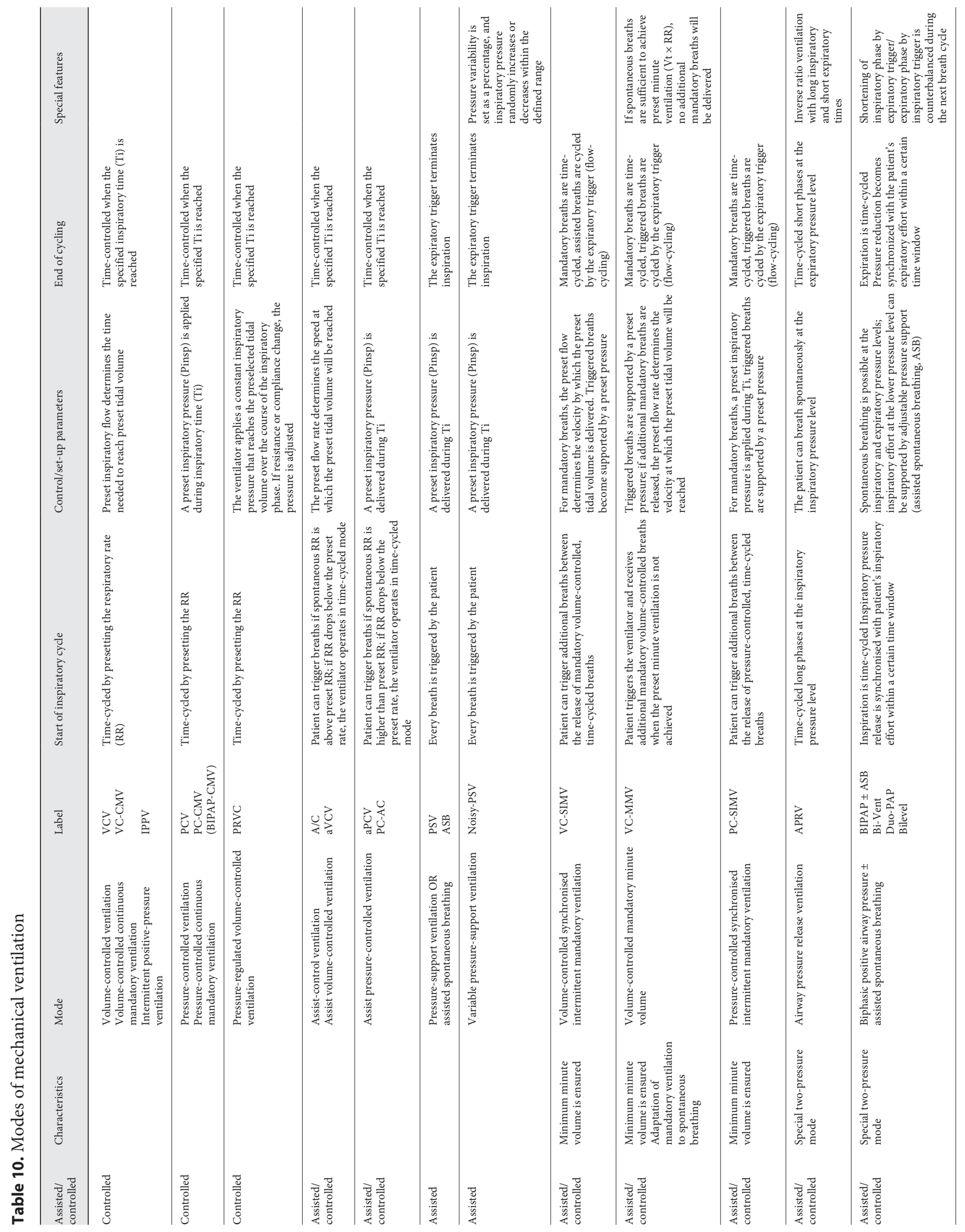




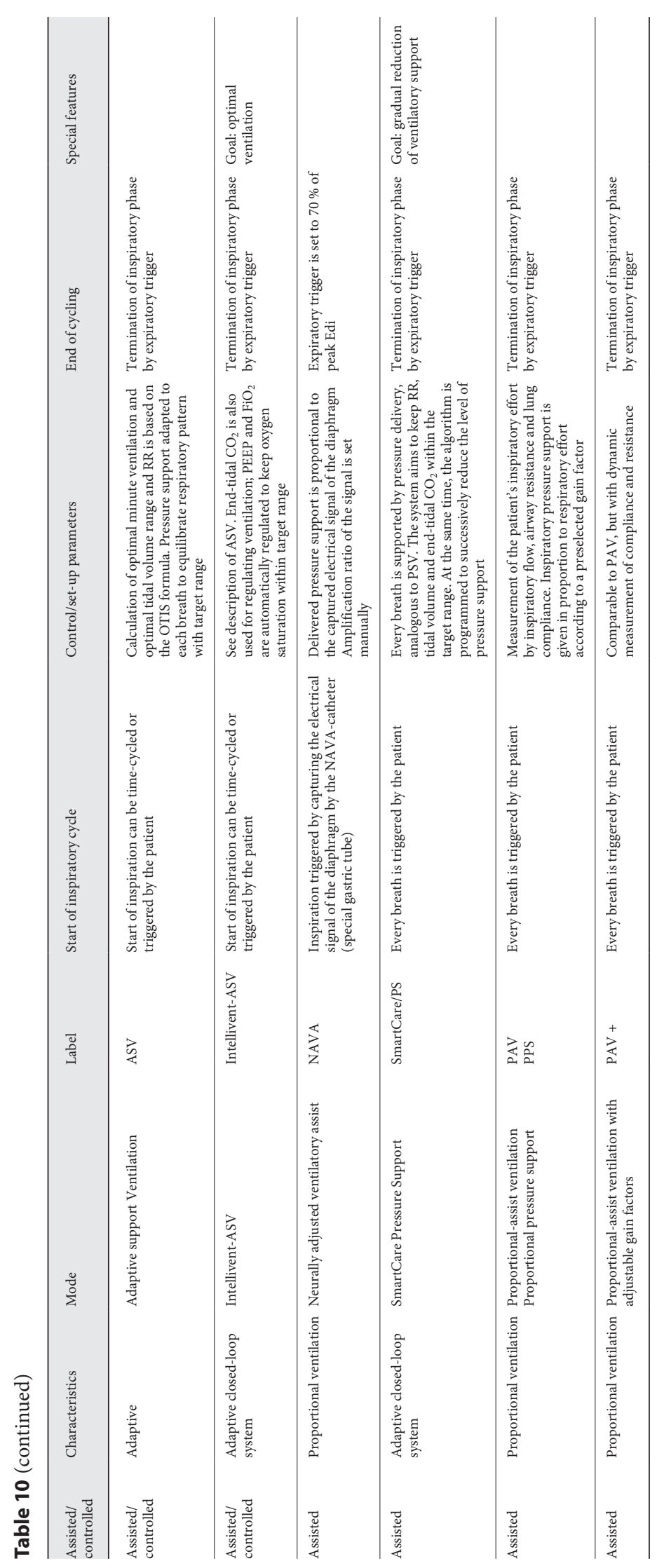

Controlled MV means that the work of breathing in passive patients is completely taken over by the ventilator. The pressure generated by the ventilator (pressure-controlled ventilation, PCV) as well as the volume support provided (volume-controlled ventilation, VCV) are controlled through the ventilator set-up, and the patient is unable to have any influence over these fixed specifications.

In deeply-sedated patients and often in muscle-relaxed patients, controlled MV leads to the ventilator taking over the entire work of breathing. If the patient is not relaxed, and only superficially sedated or even awake, then the entire work of breathing is not necessarily taken over by the ventilator. This can lead to a substantial amount of work for the patient's respiratory muscles, especially when there is no existing synchrony between the ventilator and the patient. Improved synchronisation can, however, be achieved by implementing an inspiratory trigger for the patient, like that used in assisted ventilation procedures. Assisted-controlled MV modes (assist-control ventilation [ACV] or assisted pressure-controlled ventilation [APCV]) can lead to respiratory muscle unloading, especially if the background frequency is above the respiratory rate of the patient, and the patient is then formally ventilated in a controlled manner. According to the nomenclature of the S3 Guideline "Invasive Mechanical Ventilation and Extracorporeal Membrane Oxygenation in Acute Respiratory Insufficiency" [162], this ventilation procedure does not officially deal with (purely) "controlled" ventilation, since triggering is carried by the patient, thereby allowing or taking into account the spontaneous breathing fraction of the ventilator, even though, for example, a defined tidal volume is applied onto the trigger by the ventilator (ACV).

Hybrid MV then refers to the situation where different forms of MV are combined e.g. SIMV + PSV.

Given the lack of a set nomenclature, ventilation modes that are practically identical are described differently in some instances by ventilator manufacturers. A clear description of the ventilation form should be particularly noted, even when ventilated patients are being transferred to another clinic such as a weaning centre.

\subsubsection{Controlled MV}

From a pathophysiological perspective, muscle-unloading ventilation that allows respiratory muscle rest and regeneration between spontaneous breathing phases is generally worthwhile during prolonged weaning [248]. However, the use of controlled or fully unloading forms of ventilation can also lead to structural damage of the 
respiratory muscles $[249,250]$. This so-called ventilatorinduced diaphragmatic dysfunction (VIDD) was initially described in animals [251], and subsequently confirmed in humans during the acute phase of the disease [252, 253]. A MV duration of 18-69 hours (mean duration: 34 \pm 16 hours) already leads to significant atrophy of the diaphragm muscles [252]. This atrophy, along with the loss of diaphragmatic strength, correlates with the duration of MV [254], possibly in a logarithmic fashion, whereby the loss of strength in the first hours or days of MV is the most pronounced [255].

Besides the duration of ventilation, the extent of unloading appears to play an important role in the development of diaphragmatic injury, as demonstrated in a comparative study of assisted versus controlled MV in rabbits [256]. In this study, the mRNA (messenger Ribonucleic Acid) levels of a gene known to be associated with muscle atrophy were found to be increased by $174 \%$ in the controlled-ventilation group. Additional causes of VIDD have been postulated to be oxidative stress, structural damage of the muscles, and altered muscle fibre development [66]. Therefore, the use of long-term MV in which the muscles are completely inactive leads to atrophy of the respiratory muscles [252] and does not appear to be useful for weaning therapy.

R10: Sufficient unloading of the respiratory muscles to allow regeneration between spontaneous breathing phases during weaning is recommended.

R11: Avoiding long-term MV that completely unloads the respiratory muscles is recommended.

\subsubsection{Assisted MV}

The term "assisted" means that support from the ventilator is initiated at the beginning of the inspiration phase (inspiratory trigger). Depending on the type of MV, the patient can also terminate support from the ventilator by an expiratory trigger. This is where the set-up of the inspiratory trigger can influence the patient's work of breathing. The same applies for the selection of the pressurisation rate [257], as well as an appropriate expiratory trigger (also known as "inspiratory cycling" or "off-cycling") [101, 258]. Switching between controlled and assisted ventilation means that the patient's resulting work of breathing amounts to more than $50 \%$ of the total work of breathing performed during spontaneous breathing [259].

5.3.3.1. Pressure-Support Ventilation (PSV). The most frequently implemented type of assisted ventilation is PSV. Here the patient can independently regulate the breathing pattern based on an inspiratory and expiratory

Prolonged Weaning: S2k Guideline by the

German Respiratory Society trigger. The inspiration trigger as well as the off-cycling criteria vary amongst modern ventilatory devices during assisted or pressure-support ventilation. Since fixed device settings (off-cycling at 25\% peak flow) leads to expiratory delays, especially in COPD patients [258, 260, 261], ventilators with selectable variables should be used to facilitate optimal MV. The level of pressure support during the inspiratory phase is constant and therefore independent of the patient's respiratory drive; in the expiratory phase, the airway pressure drops to zero, or to a preselected PEEP level. PSV diminishes the work of breathing and oxygen consumption in respiratory muscles and prevents diaphragmatic fatigue $[262,263]$.

R12: Ventilatory devices with adjustable inspiratory and expiratory triggers are recommended for use in pressure-support ventilation.

\subsubsection{Combined MV (synchronised intermittent}

mandatory ventilation, SIMV)

In this type of ventilation, the patient can trigger additional breaths between the time-controlled mandatory breaths. The rapid alternation between assisted and nonassisted breathing during SIMV does not appear to be answered by the respiratory centre at the same speed [264]. This means that the respiratory muscles are also active during assisted breathing, which results in overloading and fatigue of these muscles [265].

Based on this finding, the use of SIMV in prolonged weaning cannot be recommended (see Recommendation 15).

\subsubsection{Adaptive MV}

5.3.5.1. Adaptive Support Ventilation (ASV). This MV procedure is a combination of pressure-controlled SIMV and PSV. The targeted alveolar ventilation (V'A), PEEP and $\mathrm{FiO}_{2}$ values are set by the user. The degree of pressure support as well as the frequency of mandatory breaths are calculated by the system itself. The system assists spontaneous breathing and regulates this support in a way that enables the preset V'A to be reached. All of the published studies that have investigated this form of MV have been carried out in patients with simple-weaning (Group 1). Out of 8 randomised studies [266-273], 6 of these reported that extubation could be achieved sooner with the use of ASV; the mean duration of intubation was 4 hours shorter in one study [271] and less than 1 hour shorter in another [270]. The remaining 2 studies solely showed a reduction in the number of manipulations required on the patient and the ventilator [272, 273]. Furthermore, there is evidence for the potentially better unloading of 
respiratory muscles under ASV therapy [274]. A clear advantage of this was seen in a study of 97 COPD patients who had been invasively ventilated for $>24 \mathrm{~h}$ before inclusion and randomisation into ASV or PSV treatment groups (median ventilation duration prior to inclusion: 48 and 67 hours, respectively), and who fulfilled the criteria for weaning; here, the authors reported a significant reduction in the duration of weaning ( $24 \mathrm{~h} \mathrm{[20-62} \mathrm{h]} \mathrm{vs.}$

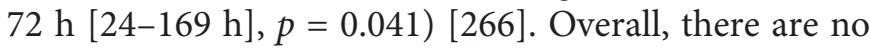
available data on the use of ASV in prolonged weaning, and no comparative studies of established weaning concepts [35, 275]. Nonetheless, it can be deduced from the existing studies that no significant benefit could be demonstrated for the control group (usually those receiving PSV).

5.3.5.2. Neurally Adjusted Ventilatory Assist (NAVA). In this form of MV, the inspiratory effort of the patient is detected via an oesophageal probe that measures electrical activity of the diaphragm. The selection of an amplification factor then regulates delivery of pressure support, which behaves proportionally to the signal obtained by electromyography. The signal acquisition also appears to be safely derivable and feasible in patients with criticalillness polyneuropathy/myopathy [276-278]. If the ventilator measures an increase in tidal volume in the presence of a constant amplification factor, then this is a sign of increasing respiratory muscle effectivity. This parameter can potentially be used as a marker for respiratory muscle recovery [279]. Another potentially valuable aspect of this technique relates to the visualisation and monitoring of electrical activity in the context of weaning $[253,279-282]$. When compared to PSV, NAVA is associated with reduced patient-ventilator asynchrony [283]. Delisle et al. were also able to show that in comparison to those undergoing PSV, patients receiving NAVA therapy had fewer ineffective trigger efforts and improved sleep quality, with significantly increased proportions of REM and deep-sleep phases, less fragmented sleep and fewer episodes of central apnea [284]. What appears to be just as important as trigger sensitivity is the synchronisation of off-cycling (the switch from the inspiratory to expiratory phase), which is often rather markedly delayed in pressure-support ventilation [285] and becomes significantly reduced through NAVA therapy. The importance of a synchronised interaction between the patient and the ventilator has been underestimated until now, but possibly has a significant causal component for the development and maintenance of prolonged weaning [286-288]. To date, few studies have investigated the role of NAVA in weaning. Compared to PSV, the use of NAVA reduces the risk of over-assistance [289]. In patients who had undergone more than 72 hours of prolonged controlled ventilation, the use of NAVA was associated with the reconditioning of diaphragm activity when compared to the effects of PSV $[289,290]$. A recently published prospective randomised study of 33 COPD patients in prolonged weaning showed that the use of diaphragmatic monitoring alone led to a significant improvement of the conventional ventilation method, and that following a switch to NAVA, $69 \%$ of patients showed improved synchronisation, a reduction in the work of breathing and increased comfort [291].

Therefore, both the monitoring of diaphragmatic activity and the use of NAVA can improve patient-ventilator interactions in COPD patients undergoing prolonged weaning. Studies relating to patients without chronic obstructive lung disease and difficult or prolonged weaning were not found.

5.3.5.3. Proportional Assist Ventilation (PAV). In proportional assist ventilation, the ventilator measures instantaneous gas flow (as a measure of inspiratory effort) and calculates the inspiratory muscle pressure (Pmus) of the patient based on measurements for elastance, resistance, and current respiratory performance. The degree of compensation (respiratory unloading) is then expressed as a percentage value. The available data on the effects of PAV demonstrate a reduction in the work of breathing in weaning patients with underlying COPD [292]. However, respiratory unloading was more effective and the intrinsic PEEP more strongly reduced when PAV was supplemented by positive-expiratory pressure. Another study reported that for an experimental increase in thoracic and abdominal restriction, support from PAV is more effective than that under PSV, since PAV adapts proportionally to the inspiratory effort of the patient [293]. Therefore, loading and unloading under PAV is generally more homogeneous than it is under PSV. Changes in resistance or compliance during MV that cannot be foreseen during ventilator set-up can lead to a socalled "run-away" phenomenon, which corresponds to over- or under-assistance of the ventilator. This problem can be dealt with by carrying out continuous measurements of resistance and compliance, as in the newer ventilation technique known as PAV+ (PAV with adjustable gain factors) [294, 295]. Because it directly adapts the breathing support to the patient's needs, PAV can effectuate a better interaction between the patient and ventilator, and positively influence sleep quality [296]. A prospective controlled randomised pilot study in which 54 (mostly intensive-care) patients with a ventilation dura- 
tion of $>36 \mathrm{~h}$ of PAV+ vs. PSV were compared (27 PAV, 23 PSV) showed no difference in the time taken until the first successful SBT, but did reveal a significantly better patient-ventilator interaction in PAV-treated patients [297]. There are currently no study data that show an advantage of $\mathrm{PAV}+$ in patients undergoing prolonged weaning, although indirect evidence does suggest that $\mathrm{PAV}$ is not inferior to PSV. A recommendation for the use of PAV can therefore not be given.

\subsubsection{Hybrid MV}

Hybrid MV combines assisted forms of ventilation that support tidal volume with assisted forms that guarantee respiratory minute volumes [298]. Modern ventilatory devices enable a multitude of possible combinations (e.g., IMV (intermittent mandatory ventilation) + PSV, IMV + ATC, intermittent mandatory pressure-release ventilation (IMPRV), BIPAP (bi-level positive airway pressure) + PSV, BIPAP + ATC, PSV + ATC and PAV + ATC).

In theory, the integration of 2 different forms of $\mathrm{MV}$ could be important for patients undergoing prolonged weaning. By adding a tidal volume-supporting procedure (e.g., PSV) to a minute breathing volume-supporting procedure (e.g., BIPAP), the degree of muscle unloading in both procedures can potentially be controlled in a specific manner. Hybrid ventilation concepts are plausible in the context of weaning. They entail the progressive reduction of BiPAP frequency with a simultaneous increase in the proportion of pressure-supported breaths. As the capacity of the respiratory muscles increases over the course of therapy, the extent of pressure support is progressively reduced and $\mathrm{MV}$ is ultimately terminated.

However, there are no studies that have clearly demonstrated that combining these ventilation processes is actually advantageous for weaning patients. In addition, the results of studies investigating the effects of SIMV (see Chapter 5.3.4) suggest that the use of hybrid ventilation methods may have disadvantages. The hypothesis for this is that an insufficient response of the respiratory centre to rapid alternation between assisted and non-assisted breaths can also be disadvantageous in hybrid ventilation when no feedback algorithm is integrated, as is the case for adaptive ventilation.

R13: The use of hybrid ventilation techniques in prolonged weaning cannot be recommended due to insufficient evidence.

\subsubsection{Monitoring}

Various methods are available for the assessment of respiratory muscle strength and endurance. The mea- surement of maximal inspiratory and expiratory pressures as well as vital capacity in patients who are breathing spontaneously via an artificial airway requires co-operation from the patient side and is predictive for the time required until weaning is successful [299]. Ultrasound of the diaphragm is becoming more recognised for its potential in predicting a patient's readiness to wean (see Chapter 5.1.8). In this respect, the mobility of the diaphragm in M-Mode [186, 300-304] and the increase the diameter of the muscle in the apposition zone of the diaphragm [120, 187, 302-307] can be investigated. The cited studies used various measurement techniques (in ventilated or spontaneously-breathing patients). Generally speaking, there is a causal relationship between diaphragm function and weaning outcome, although the predictive value varies considerably between some studies [300]. At the same time, the methodology does not appear to be feasible for all patients [300, 304]. Magnetic stimulation of the phrenic nerve with simultaneous measurement of oesophageal pressure via a catheter serves as a particularly laborious and invasive technique [308]. Such an examination does not depend on cooperation from the patient, but rather on the examiner's experience. Furthermore, this technique is rarely available and not applicable to all patients. The comparable measurement of oxygen uptake can also serve as an indicator for the level of oxygen consumption of the respiratory muscles or their degree of unloading [264]. An additional tool for assessing respiratory muscle capacity is through the measurement of "neuro-ventilatory effectiveness" [253]. This is determined by the use of an electrode attached to a NAVA ventilator, and is defined by the ratio of tidal volume to electrical activity of the diaphragm (Vt/EAdi). The cited study on this technique showed that in prolonged weaning patients, neuro-ventilatory effectiveness differs between failed and successful SBTs, without giving a clear cut-off value.

\subsubsection{Weaning Concepts Associated with the Use of}

\section{Various Forms of MV}

The goal of the weaning process is to restore the state of the respiratory muscles to the point where they can independently manage the respiratory load. This reduction in load is mainly achieved through means of effective treatment of the underlying illness or accompanying illnesses that have led to weaning failure. The restoration of respiratory muscle capacity is the second important component of the weaning process.

Respiratory muscle training or the gradual takeover of the work of breathing by the patient can take place by 

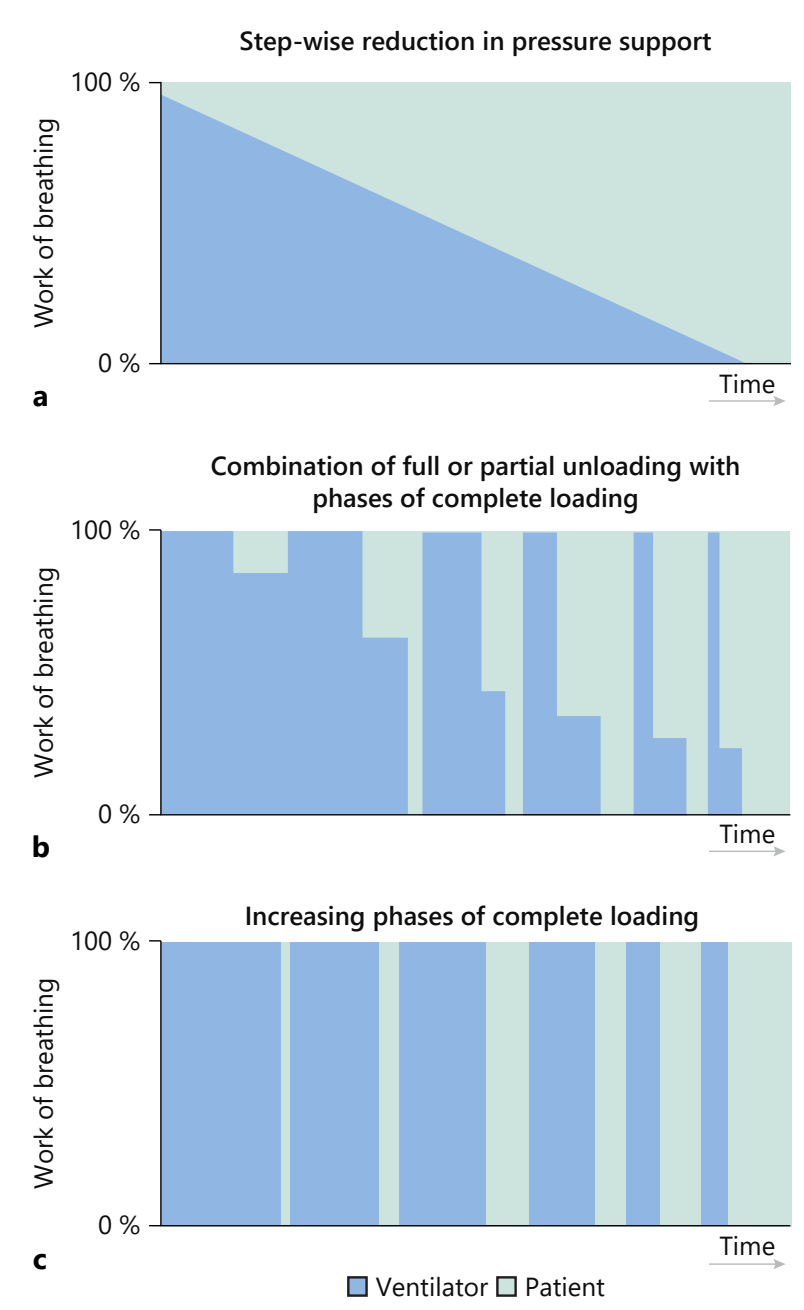

Fig. 4. Different methods for reducing ventilation support and promoting the takeover of breathing work by the patient.

continuously reducing respiratory support in the form of assisted ventilation techniques or through intermittent periods of spontaneous breathing (completely independent breathing without ventilatory support) [309]. These processes can also be combined (phases of controlled ventilation in alternation with complete rest and phases of assisted ventilation) and incorporated into an individual weaning plan (see Fig. 4).

In principle, inspiratory muscle strength training can additionally be carried out during the spontaneous breathing phases [310] in order to regenerate respiratory muscle capacity. One positive controlled study exists for this concept, although SIMV was used as the mode of MV [311].
5.3.8.1. Gradual Reduction of Support. Gradual reduction of support generally occurs in PSV mode. The required level of pressure is titrated according to clinical criteria so that the respiratory rate of the patient ranges within an area below 25-30 breaths per minute [35, 275, 312]. Furthermore, inspiratory pressure support can be reduced in a stepwise manner until a level is reached at which the artificial airway can be removed (generally $<8$ $\mathrm{cm} \mathrm{H}_{2} \mathrm{O}$ ) [85]; subsequent NIV may be necessary (see Chapter 5.6). For PSV, it should be noted that an overlysensitive expiratory trigger can increase the work of breathing. However, in patients with obstructive airway diseases, an under-sensitive expiratory trigger can lead to the development of an intrinsic PEEP, which negatively influences patient-ventilator synchrony $[101,313]$. The degree of reduction in the work of breathing can differ considerably between devices, despite identical ventilator settings [314].

5.3.8.2. Automated Adjustment of Assisted Ventilation (Automated Weaning). In this computerised closed-loop system $[315,316]$, algorithms are stored that aim to maintain specific ventilation parameters via regulation of pressure support (in PSV mode) within a target range (respiratory rate $12-28$ breaths/min; $\mathrm{Vt}>300 \mathrm{~mL}$, or $>250 \mathrm{~mL}$ when body weight is $<50 \mathrm{~kg}$ and $\mathrm{PETCO}_{2}$ is $<55 \mathrm{~mm} \mathrm{Hg}$, or $65 \mathrm{~mm} \mathrm{Hg}$ in COPD patients). In a large study cohort of preselected patients, this mode of MV was more often capable of maintaining the breathing parameters of the patient within the target range compared to cases where the standard treatment of PSV was used [315]. In a randomised multicentre study of weaning patients, this automated weaning mode was compared to a standardised weaning protocol with spontaneous breathing phases. The authors observed a reduction in weaning time from 5 to 3 days, and a reduction in the total ventilation time from 12 to 7.5 days [316]. At the beginning of the study, the patients belonged to Weaning Categories 1 and 2 (see Chapter 3 ), where only $15 \%$ of the total number of patients screened were ultimately included in the study [317]. In contrast, Rose et al. [318] could not find any advantages of the system over the conventional approach used by an established weaning centre. Another study of Category 1 and 2 patients in which an automated system was compared to a weaning protocol reported a reduction in the time until extubation (4 vs. 5 days) [319]. The system should not be used when patients: (i) have a neurological condition that influences breathing control [320]; (ii) are strongly sedated [321]; (iii) are highly agitated or have severe neuropathy or myopathy [320]. Furthermore, there appears to be a higher failure rate amongst patients 
with COPD [321]. A Cochrane analysis of automated weaning has become available in the meantime, although the studied total ventilation time of 96 hours does not fall into the category of prolonged weaning. It was concluded that automated systems are advantageous for mixed and internal medicine patient groups in Weaning Categories 1 and 2, but not for surgical patients [322].

One particular study that was not included in the aforementioned Cochrane analysis (because it was published later) is that by Taniguchi and colleagues [323]. The authors discovered that in patients undergoing simple weaning, an individually-based weaning plan is superior to an automated system in terms of weaning duration. When automated weaning was compared to protocol-based weaning in Category 1 and 2 patients, weaning duration was found to be shorter, although in the protocol arm of the study, there were a number of protocol violations on $>25 \%$ of the study days.

\subsubsection{Intermittent Loading by Disconnecting the}

Ventilator (Spontaneous Breathing) or the Reduction of Pressure Support

While the load remains relatively constant during a gradual reduction in pressure support, weaning patients handle a cyclical load through periods of spontaneous breathing, which is akin to that found in endurance training [324]; this latter process is potentially superior in prolonged weaning.

In contrast to what is described in Chapter 5.1.7, these phases of spontaneous breathing are not aimed at distinguishing between patients who are eligible for artificial airway removal, but rather serve to recondition the respiratory muscles. The length of the spontaneous breathing phases therefore tends to strongly exceed the time periods of 30/120 min that are described in Chapter 5.1.7 [325]. If the spontaneous breathing phases are sufficiently long, invasive MV can be ceased and the artificial airway removed, unless further contraindications exist. Subsequent NIV may be necessary in some cases (see Chapter 5.4, 5.6 and 6.6). While the length of the spontaneous breathing phase required in this context has not yet been systematically investigated for prolonged weaning, published data suggest time periods of up to $18 \mathrm{~h}$ [12]. These spontaneous breathing phases should be carried out daily. According to a study by Liang et al. [326], the use of music during these phases reduces heart and respiratory rates, and extends the duration of the spontaneous breathing phase. However, this effect could not be reproduced in a second, significantly larger study [327]. If muscle strength does not suffice for a conventional spontaneous breathing

Prolonged Weaning: S2k Guideline by the German Respiratory Society phase following disconnection from the ventilator, cyclic loading can be carried out by markedly reducing pressure support. Spontaneous breathing phases that each have a different degree of pressure support can lead here to alternate loading of the respiratory muscles, or to differing training intensity levels [263]. This is where it is important to note the patient's work of breathing is influenced not only by the type and size of the tracheal/endotracheal cannula $[72,76,262,328]$, but also by the choice of ventilation settings as well as the characteristics of the ventilator [329, 330]. When spontaneous breathing is carried out with inspiratory pressure support $[35,331-333]$, this can only take place with a inflated cuff. However, deflating the cuff during spontaneous breathing has the advantage of supporting secretion elimination, as well as potentially reducing the possibility of later complications of the upper airways [328, 334, 335]. During spontaneous breathing, different tracheostoma/tube attachments can be employed without pressure support, although their inspiratory and expiratory resistance can differ considerably (i.e., influence the patient's work of breathing) [336, 337]. Attachments with expiratory resistance (positive expiratory pressure, PEP valves or speech valves) may only be used with a deflated cuff.

The use of MV between spontaneous breathing phases is aimed at unloading the respiratory muscles. However, the level of unloading that produces the best effect is yet to be investigated. What the various assisted ventilation forms have in common is that a significant proportion of the work of breathing is done by the patient, despite the mechanical support. The extent of this depends not only on the degree of mechanical support and respiratory drive, but also on the individual breathing mechanics (resistance and compliance) of the patient [312]. Indeed, overloading of the respiratory muscles can lead to structural damage [338].

\subsubsection{Comparison of Different Ventilation}

Approaches and Weaning Concepts

Two large studies have compared the effects of PSV vs. SIMV on weaning outcome, and the effect of spontaneous breathing on weaning outcome $[35,275]$. The majority of patients in both studies were undergoing prolonged weaning. Brochard et al. [275] showed a reduction in average weaning duration with PSV (5.7 days), which was significant when compared to the approach using spontaneous breathing ( 8.5 days) and SIMV (9.9 days). In contrast, Esteban et al. [35] showed that intermittent spontaneous breathing trials that took place once or several times daily were associated with a reduction in average 
weaning time (3 days) when compared to that associated with PSV (4 days) or SIMV (5 days). In an additional study comparing PSV to unsupported breathing, no superiority of either approach was found [292]. In this particular study, however, patients were ventilated with PSV for partial unloading between spontaneous breathing phases. Nonetheless, a trend towards a shorter ventilation period was observed in the spontaneous breathing group (130 vs. $181 \mathrm{~h})$. The disparate findings from the first 2 studies mentioned above are likely due to the different use of weaning methods. In the study by Brochard et al. [275], up to three 2-h spontaneous breathing phases were carried out before extubation. This would have placed a considerable demand on the respiratory muscles and likely prolonged the duration of weaning. In contrast, the patients investigated by Esteban et al. [35] were already extubated after the first successful 2-h trial.

Patients who required long-term MV ( $>21$ days) and were treated in a long-term care facility (comparable to a weaning centre) were able to switch between unassisted spontaneous breathing and ACV via a tracheostoma more quickly than with PSV after an unsuccessful weaning attempt with intermittent weaning. A difference in lethality was not observed at either 6 or 12 months' postweaning [339]. In general, PSV can be used as a weaning method, although the approach via spontaneous breathing is potentially superior if the number of daily spontaneous breathing phases are initially limited to 1-2 episodes over a maximum of $2 \mathrm{~h}$, thus protecting the respiratory muscles from imminent fatigue. As the weaning process progresses, these breathing phases can of course be expanded under clinical observation and with blood gas monitoring (e.g., hypercapnia can indicate overloading of the respiratory muscles) until transfer to NIV or completely unassisted breathing is possible.

R14: A gradual reduction in assisted ventilation or intermittent assisted/non-assisted spontaneous breathing phases can each be used in the weaning process.

$R 15$ : The use of SIMV in prolonged weaning is not recommended, since this mode of ventilation is associated with an unacceptably high work of breathing.

\subsubsection{The Role of Continuous Positive Airway}

Pressure in Weaning

The use of continuous positive airway pressure (CPAP) does not provide mechanical pressure support; therefore, strictly speaking, it is not a form of MV. Nonetheless, it does play a certain role in weaning. The use of CPAP conditions the patient for spontaneous breathing and can be implemented during the spontaneous breathing trials both for examining the patient's readiness to extubate (Chapter 5.1.7) as well as for reconditioning the respiratory muscles (Chapter 5.3.9).

CPAP in obstructive lung disease: CPAP increases intrathoracic pressure and minimises respiratory effort by shifting the load to the expiratory muscles [340]. During spontaneous breathing, the work of breathing is lowered as the level of CPAP increases [341], with a simultaneous reduction in dyspnoea. The use of CPAP therapy in obstructive lung disease can be worthwhile, since CPAP takes over the mechanism of the pursed lip-breathing technique that is otherwise deactivated by the artificial airway. This also leads to a reduction in trigger-associated work of breathing [342]. Application of CPAP can also reduce intrinsic PEEP in obstructive lung diseases, whereby the length of the SBT is significantly prolonged [343, 344].

CPAP prior to scheduled extubation: CPAP reduces the RSBI during an SBT [174]. Despite this it appears that pressure support during these SBTs does not have any influence on the failure rate following extubation (see Chapter 5.1.7) [174, 184, 185].

CPAP in atelectasis: CPAP was already implemented very early on for use in patients who had undergone abdominal surgery [345]. The use of CPAP therapy during this phase significantly reduces the risk of atelectasis and pneumonia and thereby lowers the rate of reintubation [346].

CPAP in cardiac failure: CPAP has a positive effect on pre- and after-loading of the left ventricle, so that spontaneous breathing can only be made possible with CPAP, particularly in the case of left-sided heart failure [84].

In general, the use of CPAP is possible for reconditioning the respiratory muscles during SBTs. One study provides a specific pressure range of up to $5 \mathrm{~cm} \mathrm{H}_{2} \mathrm{O}$ [35]. Given that there are advantages associated with deflating the tube/tracheostoma cuff during the spontaneous breathing phase $[328,334,335]$ in prolonged weaning, the use of CPAP during this period is excluded.

\subsection{The Ventilation Interface}

There is a characteristic range of indications, as well as specific advantages and disadvantages, associated with invasive MV with an endotracheal tube or a tracheal cannula versus NIV with various masks (Table 11).

\subsubsection{NIV Interfaces}

The advantages and disadvantages of various ventilation interfaces in NIV are presented in Table 12. Further detailed explanations on NIV interfaces can be found in 
Table 11. Advantages and disadvantages of airway interfaces (modified according to [16])

\begin{tabular}{|c|c|c|c|}
\hline & $\begin{array}{l}\text { Translaryngeal } \\
\text { endotracheal tube* }\end{array}$ & Tracheotomy & Non-invasive airway interface \\
\hline Advantages & $\begin{array}{l}\text { Reduced rate of aspiration } \\
\text { No leakage } \\
\text { Suction and respiratory toilet possible } \\
\text { Simple and extensive monitoring possible } \\
\text { Maintenance of airway patency } \\
\text { Constant pressure } \\
\text { High ventilation pressure possible } \\
\text { No spontaneous breathing/defensive } \\
\text { reflex necessary }\end{array}$ & $\begin{array}{l}\text { See Translaryngeal endotracheal tube* - plus: } \\
\text { Reductions in dead space, airway resistance } \\
\text { and work of breathing } \\
\text { Improved patient comfort } \\
\text { Reduced/no sedation } \\
\text { Maintenance of glottis function with the } \\
\text { reduced risk of aspiration } \\
\text { Transfer from the ICU to a specialised } \\
\text { ward possible }\end{array}$ & $\begin{array}{l}\text { No damage to airways } \\
\text { No or only minimal sedation required } \\
\text { Intermittent use possible } \\
\text { Maintenance of ability to communicate } \\
\text { Maintenance of cough clearance } \\
\text { Oral food intake }\end{array}$ \\
\hline Disadvantages & $\begin{array}{l}\text { Tube-associated infections } \\
\text { Reduced secretion and cough clearance } \\
\text { Deep sedation necessary } \\
\text { Increased resistive work of breathing } \\
\text { Damage to the upper airways, vocal cords } \\
\text { and larynx } \\
\text { Unplanned extubation } \\
\text { Tube malposition } \\
\text { No oral food intake } \\
\text { Sinusitis (nasal tube) } \\
\text { Scar formation }\end{array}$ & $\begin{array}{l}\text { Early complications } \\
\text { Local wound infections } \\
\text { Reduced cough clearance (less pronounced as } \\
\text { in endotracheal tube patients) } \\
\text { Problems with communication } \\
\text { Complications from invasive procedure } \\
\underline{\text { Late complications }} \\
\text { Tracheal scar strictures or granulation tissue }\end{array}$ & $\begin{array}{l}\text { Leakage with the loss of PEEP } \\
\text { Risk of aspiration } \\
\text { Limited monitoring of applied breath } \\
\text { volume } \\
\text { Insufficient effectivity from reduced } \\
\text { compliance } \\
\text { Local complications (pressure sores, } \\
\text { conjunctivitis, aerophagy) }\end{array}$ \\
\hline
\end{tabular}

Cited literature: [24, 25, 71, 347-357].

Table 12. Advantages and disadvantages of NIV interfaces: + advantage; 0 neutral; - disadvantage [16]

\begin{tabular}{llll}
\hline Facet & $\begin{array}{l}\text { Nasal } \\
\text { mask }\end{array}$ & $\begin{array}{l}\text { Full-face } \\
\text { mask }\end{array}$ & Helmet \\
\hline Mouth leakage & - & + & + \\
Volume monitoring & - & + & - \\
Initial response of blood gases & 0 & + & 0 \\
Speech & + & - & - \\
Expectoration & + & - & - \\
Aspiration risk & + & 0 & + \\
Aerophagy & + & 0 & 0 \\
Claustrophobia & + & 0 & 0 \\
Dead space (compressible volume) & + & 0 & - \\
Noise and irritation to hearing & + & + & - \\
Duration of use & 0 & 0 & + \\
\hline
\end{tabular}

the corresponding sections of the S3 Guideline "Non-invasive Ventilation in Acute Respiratory Failure" [16].

\subsubsection{Invasive Ventilation Interfaces}

5.4.2.1. Endotracheal Tube. Endotracheal tubes are almost always used as the primary invasive orotracheal ventilation interface in intensive care medicine. In prolonged weaning, however, they carry a number of disad- vantages when compared to tracheal cannula and NIV (Table 11). Similar to the situation with tracheal cannula, deflating the endotracheal tube during spontaneous breathing phases can lead to a further decrease in the work of breathing; however, the lack of protection against aspiration makes it problematic [358].

Subglottic accumulation of oropharyngeal secretions can occur above the cuff, and move from there into the deeper airways; this especially holds true in patients with a pre-existing swallowing disorder. Some of the newer varieties of endotracheal tubes and tracheal cannula offer the possibility of suctioning subglottic secretions. In 2013 the Hospital Hygiene and Infection Prevention Commission from the Robert Koch Institute (KRINKO) recommends the use of subglottic suctioning when the duration of invasive $\mathrm{MV}$ is expected to exceed $72 \mathrm{~h}$, in order to avoid ventilator (or tube)associated pneumonia (VAP) [359]; however, the evidence for this is weak. Although the results of 2 metaanalyses that were carried out in 2016 on patients with translaryngeal intubation did indeed demonstrate a comparably low VAP rate, the use of subglottic suctioning did not have any effect on the duration of MV, the length of ICU and hospital stays, or mortality [360, 361]. Moreover, the patients analysed in these metaanalyses were not primarily those undergoing pro- 
longed weaning. Therefore, at this stage no general recommendation for the use of endotracheal tubes with subglottic suctioning can be made for patients undergoing (expected) prolonged weaning (Chapter 5.4.3).

Since the value of other preventative measures for reducing the incidence of tube-associated pneumonia (e.g., selective oral/supraglottic decontamination and antiseptically impregnated tubes to reduce biofilm formation) is not generally applicable to the prolonged weaning setting, it is currently not possible to provide a corresponding recommendation.

R16: To reduce tube-associated respiratory effort, the use of a tube with the largest possible lumen is recommended for patients receiving prolonged ventilation.

5.4.2.2. Tracheotomy. To minimise the complications associated with the use of an endotracheal tube during long-term ventilation, and to facilitate weaning the patient from invasive MV [362] (Table 11), a tracheotomy with a change-over to a tracheal cannula as the ventilation interface can be considered if a longer period of invasive MV is needed without the option for early extubation with subsequent NIV (Table 11). This corresponds to global routine practice in intensive care medicine, where most ventilated patients are tracheotomised only after more than 10 days of invasive MV [363-365]. Besides the avoidance of (endotracheal) tube-associated side-effects, the creation of a tracheostoma allows the extension of intermittent SBTs during wakefulness [35], which occupy up to $50 \%$ of the entire ventilation time [6]. In addition, every orotracheal reintubation that follows a failed extubation is linked to an increased risk of both morbidity and mortality [20].

Most of the tracheostomata that are subjectively well tolerated enable a low level of sedation that aims for an alert and co-operative patient, which in turn eases the intermittent spontaneous breathing phases, without having to renounce on a secure access to the airways.

Additional pathophysiological advantages of the tracheal tube over the translaryngeal tube arise from reductions in dead space volume, airway resistance, as well as the work of breathing associated with these changes [71, $72,349,366]$. Furthermore, the reduced need for sedation leads to an improved capability for mobilisation and communication, as well as easier mouth, throat and tracheal care and earlier enteric or even oral alimentation [347, 367, 368].

The above-mentioned advantages are offset by the short-term complications associated with tracheotomy, such as bleeding and local infection risk $[369,370]$. The lethality risk associated with tracheotomy is difficult to estimate due to an assumed number of unreported cases; however, according to 2 systematic reviews of significant magnitude, this risk ranges from 1:600 to 1:300 [371, 372]. Symptomatic tracheal stenoses that occur after percutaneous or surgical tracheotomies and have an incidence of $0.6-2.6 \%$ have been identified as a long-term complication [373]. Swallowing disorders have also been described as a short- and long-term complication of tracheotomy [374, 375] (see Chapter 5.6.1.5).

On the other hand, prolonged translaryngeal intubation also leads to an increased risk of laryngeal and tracheal damage [357] as well as a higher infection risk for the lower airways [376]. An increased mortality rate has also been linked to extubation failure [20], where an increased number of unreported cases can also be assumed here.

5.4.2.2.1. Tracheotomy Techniques. In addition to surgical tracheotomy (ST), various percutaneous dilatational procedures have been established over the last 30 years, and can be performed at the bedside by intensive care specialists [377-379]. Until now, there are no standardised general recommendations for a specific method. The percentage of surgical tracheotomies performed in intensive care medicine is around $33-50 \%$ and mainly associated with neurological diseases [380, 381].

Compared to earlier comparative complication analyses between open surgical and percutaneous dilatational tracheotomy (PDT), newer studies generally describe fewer complications for PDT [382-386]. When compared to surgical tracheotomy, PDT is associated with fewer wound infections and bleeding complications, as well as reduced scar formation. Further advantages of PDT are the shorter time in surgery and the lower costs [382]. Surgical tracheotomy has fewer complications in terms of de/recannulation, as well as fewer obstructions of the tracheal cannula.

Accidental dislocation and scheduled tube exchange should absolutely be avoided in the first 7-10 days of PDT, since an attempt at recannulation due to uvular deviation (the so-called "backstop phenomenon") can lead to paratracheal cannula malpositioning ("via falsa") and subsequent loss of the artificial airway, with life-threatening consequences. For this reason, a difficult airway is a contraindication for PDT and an indication for surgical tracheotomy.

5.4.2.2.2. Percutaneous Dilatational Tracheotomy (PDT). Minimally invasive techniques have prevailed in the meantime over the surgically formed tracheostoma [384]. Currently there are 6 different methods for PDT, which are presented in Table 13. 
Table 13. Currently available methods for PDT [387-393]

\begin{tabular}{|c|c|c|}
\hline Year & Author & Method description \\
\hline 1985 & Ciaglia & $\begin{array}{l}\text { Applying bougienage with synthetic dilators of increasing diameter via a Seldinger } \\
\text { guidewire (multiple dilatation tracheotomy, MDT) }\end{array}$ \\
\hline $\begin{array}{l}1989 \\
1990\end{array}$ & $\begin{array}{l}\text { Schachner } \\
\text { Griggs }\end{array}$ & Dilatation with guide wire dilatation forceps (GWDF) \\
\hline 1997 & Fantoni & $\begin{array}{l}\text { Retrograde dilatation and insertion of the tracheal cannula using a turning manoeuvre } \\
\text { (translaryngeal tracheotomy, TLT) }\end{array}$ \\
\hline 2000 & Byhahn & $\begin{array}{l}\text { Cone-shaped one-step dilator (Blue RhinoTM, single-step dilatation tracheotomy, } \\
\text { SSDT) }\end{array}$ \\
\hline 2002 & Frova & Self-tapping screw dilator (PercuTwistTM, rotational dilatation tracheotomy, RDT) \\
\hline 2005 & Zgoda & Dilatation balloon (Blue DolphinTM, balloon dilatation tracheotomy, BDT) \\
\hline
\end{tabular}

Even though the one-step dilatation technique is the most frequently used method in Germany, and 2 metaanalyses showed that it has slight advantages over other techniques $[383,394]$, the currently available data do not allow a general recommendation to be made.

Since the beginning of the 1990s, video-bronchoscopic control of PDT has been required, irrespective of the technique used [395-397]. However, even with this form of monitoring, complications cannot be completely avoided. One study described bronchoscopic-induced obstruction of the respiratory tract, which was accompanied by increased auto-PEEP, air-trapping, and high ventilation pressures as well as hypercapnia and hypoxemia [398]. Furthermore, airway stenosis and needle perforation of the bronchoscope are also possible and require particular caution [371, 399, 400].

Since the first description of ultrasound-guided PDT in 1999 [401], additional publications followed, which were summarised in a review by Alansari et al. [402]. Ultrasound was described here as an alternative method to bronchoscopy. Together with the results of an RCT by Gobatta et al. [403], this analysis showed that as a means of establishing a PDT, ultrasound is equivalent to bronchoscopy in terms of its effectiveness and safety. Several groups such as Sangwan et al. [404] have also described modified techniques that use both of these imaging methods.

In Germany, bronchoscopy-guided PDT is deemed to be the gold standard in clinical practice, while ultrasoundguided PDT represents the exception. By enabling the examiner to visualise the procedure, video-bronchoscopy offers more security than a simple bronchoscopy [405, 406]. A routine preliminary ultrasound examination of the neck for site localisation and exclusion of a larger pretracheal blood vessel also appears to be clinically useful.

Due to a high shrinkage tendency, dilatative tracheostomata that have been created by a minimally invasive technique frequently close within a short time following removal of the tracheal cannula, which is desired in most cases. If there is uncertainty about the success of decannulation, a tracheostomy retainer can be inserted into the tracheostoma to prevent its premature closure; this also serves to support secretion management and ensure effective NIV (see Chapter 5.4.5).

A PDT can be quite stable, especially if it exists for a longer period, and can then also be utilised as a long-term ventilation interface in the home ventilation setting. A tracheostoma is deemed clinically stable when the tracheal cannula can easily and safely be exchanged without airway obstruction or the tendency to collapse. There are currently no studies that allow a temporal definition. Despite the obvious advantages of tracheotomy, around $40 \%$ of all patients who are ventilated via tracheal cannula develop dysphagia, even in the absence of an underlying neurological disorder. This, in turn, prolongs ventilation time or the decannulation process, and subsequently extends the weaning process [374].

R17: Due to its tendency to shrink, PDT is recommended mainly for patients with a high chance of weaning success.

5.4.2.2.3. Surgical Tracheotomy. The surgical approach essentially consists of an alternative between non-plastic tracheotomy and a plastic, epithelialised tracheostoma $[407,408]$. Non-epithelialised tracheostomata should be avoided in surgical tracheotomy, due to a higher risk of stenosis development. A primarily epithelialised trache- 
ostoma (e.g. Bjork flap [409], or vertical incision with suturing along the edge of the skin incision) should be implanted instead.

If unsuccessful weaning leads to prolonged invasive MV that will take place in a home environment or nursing facility, a stable tracheostoma created surgically or dilatatively is required for safe management of the cannula. For more information on this topic, please refer to the corresponding guidelines on the indication, organisation, and implementation of HMV [3].

5.4.2.2.4. When to Perform a Tracheotomy. The optimal timepoint to perform a tracheotomy remains controversial. It has long been standard clinical practice to tracheotomise patients on prolonged MV no earlier than 10-14 days of ventilation, if not longer [410]. The time period between intubation and tracheotomy has become shorter in the meantime, particularly because of the increased prevalence of PDT [365] [411-415]. There is indirect evidence in the literature that a tracheotomy should be performed no later than 14-21 days after invasive ventilation onset [362, 416, 417], since an even later time point is associated with an increased mortality rate. Although the literature does distinguish between so-called early and late tracheotomies, a uniform temporal definition is lacking. In the corresponding studies, early tracheotomy was defined as that occurring within 7 days of intubation, while late tracheotomy was considered to occur 8-10 days after intubation. In this guideline, early tracheotomy is defined as being carried out on post-intubation day $4-8$.

In the three largest, highest-quality randomised studies, early tracheotomy was performed within 4 days [ 418 , 419] or 6-8 days after intubation [420]. None of these studies, which had a total number of 1,667 patients, showed any short- or long-term survival benefits at either of these 2 time points in a mixed patient population. In the largest tracheotomy study so far (Young et al. [419]), only $44 \%$ of patients in the late tracheotomy group were tracheotomised de facto.

Numerous meta-analyses of tracheotomy timing have produced highly heterogenous findings based on different patient populations [421-428]. Two metaanalyses published in 2015 yielded controversial results regarding long-term mortality $[429,430]$. Whereas the meta-analysis of Hosokawa et al. [430] of 12 RCTs with a total of 2,689 patients demonstrated significant advantages of early tracheotomy in terms of ventilationfree days, duration of intensive care therapy and sedation, and the 2-month mortality rate, the meta-analysis by Siempos et al. [429] concluded that early tracheoto-

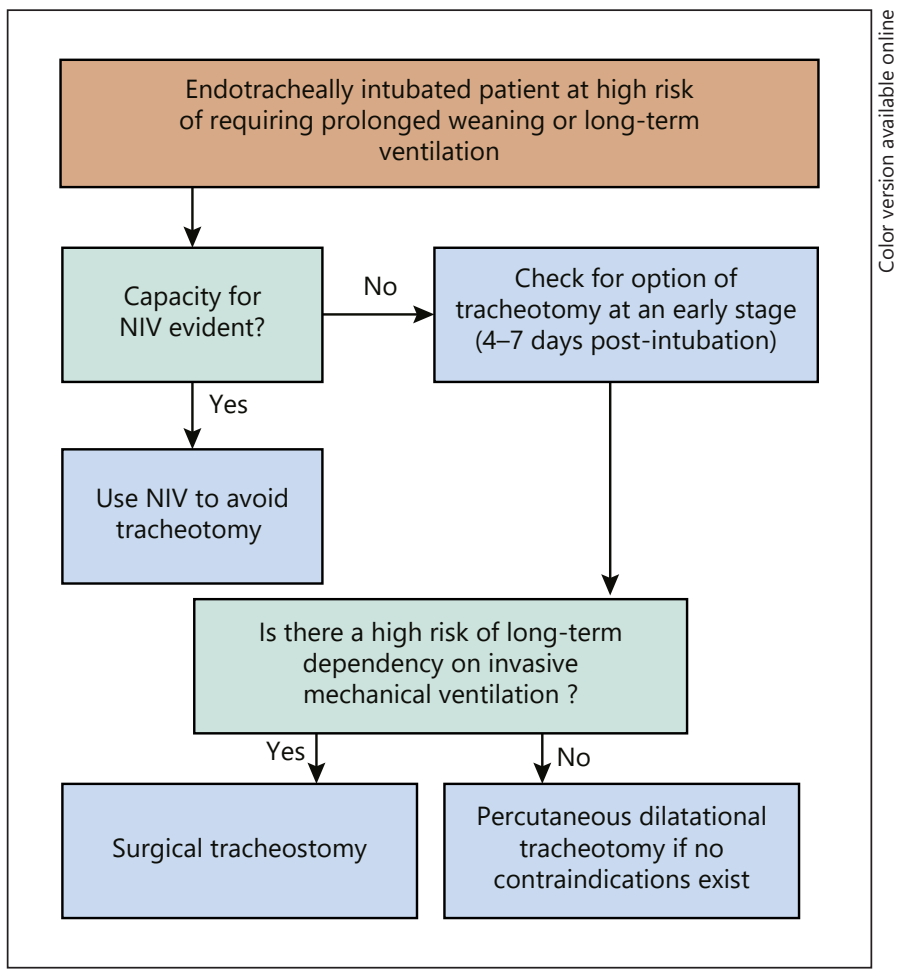

Fig. 5. Algorithm outlining the tracheotomy procedure with previous examination of NIV capability.

my had a significantly favourable influence on the duration of ventilation only, and did not affect the 3-month or 1-year mortality rates.

Despite the evidence that a tracheotomy can potentially shorten ventilation time or prevent prolonged weaning, it is currently not possible to provide a recommendation for routine early tracheotomy due to inconsistent findings of a clear overall clinical benefit, the potential tracheotomy-associated complications, and the danger of over-treatment. Therefore, the anticipation of prolonged weaning is not associated with a compelling indication for (early) tracheotomy at this point in time. Accordingly, there is a need for further research on the optimal time point for tracheotomy in special sub-groups at high risk of prolonged weaning. The decision about the timing of a tracheotomy thus remains to be made on an individual basis. Moreover, after 4-7 days of invasive ventilation, it should always be critically assessed how high the probability of prolonged weaning is, and whether extubation with subsequent NIV is a possible alternative (see flowchart in [Fig. 5]). 
Table 14. Options for improving speaking capacity in tracheotomised patients

a Under active MV

- Deflation of the tube while adjusting the ventilation parameters to ensure sufficient ventilation with an unblocked tube

- Use of an in-line speaking valve (e.g., Passy-Muir valve) with a deflated cuff only

- Use of a Blom ${ }^{\mathrm{TM}}$ tube

- Increasing the positive end-expiratory pressure (PEEP) [434]

b Under spontaneous breathing

- Sealing the opening of a deflated tube with a gloved finger

- Use of a speaking valve (one-way valve) with a deflated tube, taking contraindications into consideration (Caution: overinflation); if needed, with supplementary oxygen insufflation through a special inlet on the speaking valve

R18: A stable tracheostoma is recommended for longterm home invasive ventilation.

\subsubsection{Management of Tracheal Cannula}

After a PDT has been created, tracheal cannula should neither be changed nor removed before post-operative days $7-10$, due to the initial instability of the entry point and potential bleeding complications. If dislocation of the tracheal tube unexpectedly occurs before this period, access to the orotracheal airway should be favoured in an emergency, and, if required a bleeding tamponade of the tracheostomy should be performed. If necessary, the tracheostoma can be recannulated in 2 stages after securing the orotracheal airway, and a new tracheal cannula can be positioned.

While selected tracheal cannula with subglottic suctioning are available [431], there is currently a lack of clinical studies that demonstrate their clinically-relevant advantages in prolonged weaning patients. Due to the varying anatomy of patients who are ventilated via a tracheostoma, an individualised tracheal cannula selection that goes beyond the function of subglottic suctioning is always required to ensure optimal cannula positioning and hence the prevention of long-term tracheal complications such as granulation tissue or bleeding from endotracheal suctioning. As already discussed for endotracheal tubes in Chapter 5.4.2.1, and based on the evidence available for patients in prolonged weaning, no general recommendation for the use of tracheal cannula with subglottal suctioning can be given at present.

The accumulation of secretions on the inner wall of the tracheal cannula cannot be predicted with certainty and leads to an increase in airway resistance, that is, addition-

Prolonged Weaning: S2k Guideline by the German Respiratory Society al respiratory effort during spontaneous breathing [366]. The tube must therefore be continuously visually monitored and either cleaned or replaced. If necessary, a tracheal cannula with an easy-to-clean inner cannula can be used, taking into account the potential increase in the work of breathing due do the smaller inner lumen. Fenestrated tubes are useful for supporting existing speaking function, because when correctly positioned in the trachea, they not only allow air to pass by the cannula, but also to escape through the fenestrations in the direction of the larynx, thereby enabling or facilitating speech. The newly developed Blom ${ }^{\circledR}$ tracheotomy cannula is fenestrated, equipped with a cuff, and enables speaking due to specially-configured pressure and flap valves, even when the cannula is blocked [432]. During the inspiratory phase of the ventilator, the flap valve opens while the pressure valve simultaneously closes, thus closing the phonation port of the cannula and enabling effective ventilation. During the expiration phase, the flap valve closes and the pressure valve collapses, releasing the phonation window and enabling speech under ventilation. In a study comparing a tracheal tube with a Blom ${ }^{\circledR}$ valve, a tracheal cannula without an inner cannula, and a Passy-Muir ${ }^{\circledR}$ oneway tracheotomy cannula speaking valve, no differences in the risk of aspiration was found between the 3 versions [432]. Another study on verbal communication in tracheotomised patients also showed no significant advantages of the Blom ${ }^{\circledR}$ tube compared to the Passy-Muir ${ }^{\circledR}$ one-way tracheotomy tube speaking valve [433].

In principle, there are various ways of promoting speech function in tracheotomised patients undergoing prolonged weaning, whether they be mechanically ventilated or spontaneously breathing patients. These options are presented in Table 14. Deflation of the cuff always requires that there is no strong tendency for aspiration.

When using speaking valves in the weaning process, the effects on the work of breathing must be considered, which can differ by a factor of 4 , depending on the type of speaking value used [435]. Sub-optimal conditioning of the inhaled air should also be taken into account in the presence of secretion problems. Possible solutions to this could be the combined use of an HME filter and speaking valve (since the humidification function does not operate during speaking), as well as limiting the duration of speaking valve use, with a subsequent change to an HME filter [436].

As long as there is no risk of aspiration, the tracheal cannula can be deflated during spontaneous breathing to reduce the work of breathing [358]. Furthermore, a deflated tracheal cannula facilitates verbal communication 


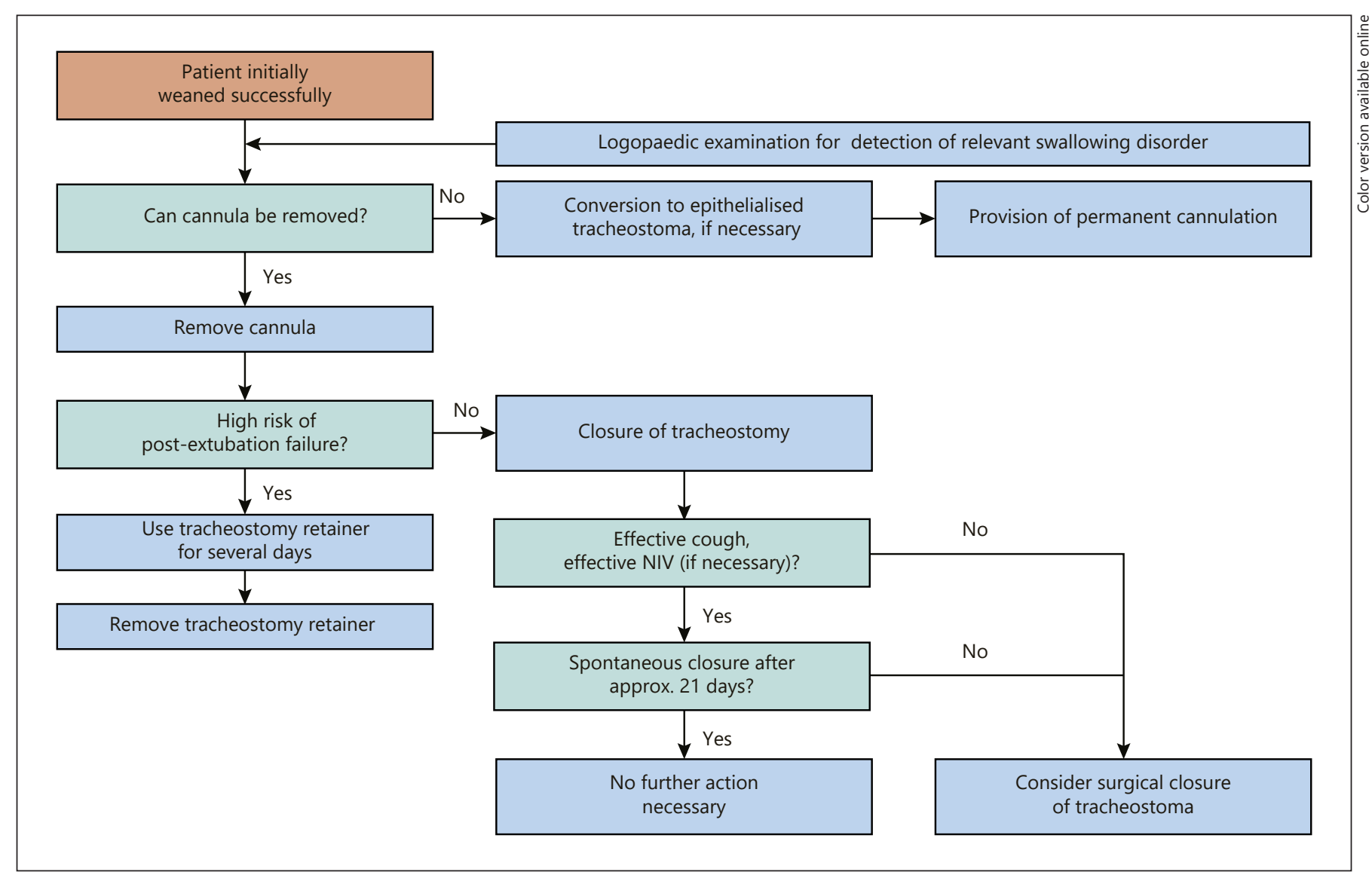

Fig. 6. Algorithm for definitive decannulation.

in the weaning process, which is important from a psychological perspective.

The effect of coughing and the associated bronchial secretion clearance can also be improved by deflating the tracheal cannula. If the clinical status of the patient allows for the tracheal cannula to be removed during spontaneous breathing, with simultaneous sealing of the tracheostoma the effectiveness of coughing can be further increased compared to that with a deflated tracheal cannula.

Speech therapy plays a central role in the assessment and improvement of speaking and swallowing function in tracheal cannula and secretion management. Where possible, consultation with an expert in speech therapy should be incorporated into this treatment process right up until the point of decannulation (Fig. 6).

R19: Provided there is no tendency for aspiration, deflating the tracheal cannula during spontaneous breathing phases is recommended to reduce the work of breathing and improve speaking function

\subsubsection{Decannulation}

The following conditions are required for definitive decannulation:

- Clinical stability

- Sufficient spontaneous breathing ability, or NIV compliance, respectively

- Lack of significant dysphagia or aspiration tendency

- Sufficient cough or non-invasive secretion management

- Cooperative patient (e.g., no delirium)

- Exclusion of an obstruction in the area of the glottis/ larynx/trachea

- Positive cuff-leak test, if necessary (compare to Chapter 5.1.10)

No consistent consensus exists regarding the methodology for decannulation and the transition to spontaneous breathing or NIV. Without being able to derive generally-applicable recommendations, a survey revealed that unless there is spontaneous regression of the trache- 
ostoma after removal of the tube, either a tracheostomy retainer or tracheal cannula with decreasing diameters are used [437].

\subsubsection{The use of Tracheostomy Retainers}

If there are concerns about performing decannulation in patients already weaned from the ventilator, or there is evidence of respiratory insufficiency with either a temporary or persistent indication for NIV (see Chapter 5.5.1), there is the option of keeping the tracheostoma patent for a few days of spontaneous breathing with the aid of a tracheostomy retainer $[328,438]$, a button, or a very thincalibre tracheal cannula (also see "Mini-tracheotomy" in Chapter 5.6.1.4; Fig. 6). Besides the correctly-inserted tracheostomy retainer allowing the additional suction of secretion, it also means that the tracheostoma canal remains open to recannulation if invasive MV becomes necessary again. Following application of a closure device, an endoscopic inspection of its position is useful for the early detection of complications (i.e., stenosis, tracheomalacia, edema, protrusion of the device into the trachea). In patients in the advanced stages of weaning who have a stable tracheostoma and no significant swallowing disorder, alternating between a tracheostomy retainer during the day and the insertion of a tracheal cannula at night for invasive ventilation can be considered on a caseto-case basis, when short-term MV is foreseeable due to NIV intolerance.

Regardless of the decannulation technique used, following closure of the tracheostoma, upper airway resistance and the work of breathing can both decrease and increase depending on the anatomical conditions. Local complications at the tracheostoma opening (e.g. ruptured cartilage rings or granulation tissue), and edema in the region of the larynx can particularly lead to decannulation failure [328].

R20: In the case of uncertainty about the success of decannulation, the use of a tracheostomy retainer is suggested to enable recannulation of the tracheostoma.

\subsubsection{Closure of the Tracheostoma}

5.4.6.1. Spontaneous Closure. After tracheotomised patients have been weaned from the ventilator and spontaneous breathing (or NIV in the case of chronic respiratory failure) is verified, the tracheal cannula or tracheostomy retainer can be removed. PDTs close completely within a short period of time (generally without the need for further intervention). Primary-epithelialised tracheostomata that are surgically inserted often require plastic surgical closure.

Prolonged Weaning: S2k Guideline by the German Respiratory Society
5.4.6.2. Surgical Closure/Plastic Wound Closure. If the tracheostoma does not shrink within 14 days of tube removal, a bronchoscopic examination of the larynx and trachea is required to rule out subglottic stenosis as the cause of the delayed or absent closure. Closure of the tracheostoma by means of plastic surgery can be considered after a period of around 21 days in cases where an insufficient level of shrinkage leads to ineffective cough and impaired speech formation. Whether NIV is paused for a few days after tracheostoma closure to prevent complications such as wound dehiscence or mediastinal/skin emphysema is dependent both on the patient's clinical status and ventilatory situation, and is hence an individual decision. An algorithm for definitive decannulation is presented in Figure 6. When dysphagia is suspected or already present, please refer to the corresponding section on "Management of dysphagia" (Chapter 5.6.1.5).

R21: If the tracheostoma does not shrink 2-3 weeks after tube removal, plastic surgery can be considered as a means of closure if underlying complications have been ruled out.

\subsubsection{Complications following Decannulation}

The development of tracheal stenosis after long-term intubation or tracheotomy is a complication with an expected occurrence of approximately $10-20 \%$ of clinically relevant stenoses (>20\% lumen constriction) [416]. This can either be a fixed, scarring type of stenosis, or tracheal instability due to tracheomalacia. Endoscopic examination also frequently identifies so-called complex stenoses. Such stenoses are not uncommonly the cause of weaning failure or the need for recannulation. Pre-existing but clinically-asymptomatic stenoses of the trachea, such as those due to intrathoracic goitre, can additionally become a problem of their own during the weaning process.

Performing a spirometry test in cooperative patients (at the bedside with a pocket spirometer, if necessary) can be helpful for assessing the stenosis and monitoring its progression.

\subsubsection{Bronchoscopic Monitoring After}

Decannulation

Tracheal damage after tracheotomy is not a rare event. Cartilage ring fractures, the development of tracheomalacia/tracheal stenosis, or swelling in the region of the larynx can all impede or prevent successful decannulation and affect subsequent steps such as the transition to NIV. The latter may be caused by tracheostomata that are too highly placed, especially when a dilatative tracheotomy is performed without careful bronchoscopic identification 
of the correct puncture site under the 1st, or preferably 2nd, tracheal ring. The first test for an existing tracheal stenosis that can be performed after tracheal cannula removal is to seal the tracheostoma with a gloved finger: if stridor occurs then bronchoscopic examination of the trachea should take place before definitive removal of the cannula. Here, translaryngeal access is superior to the tracheostoma and hence advantageous for judging the laryngotracheal junction. To determine the associated pathology, the ensuing approach should be discussed on a case-by-case basis amongst intensive care specialists, thoracic surgeons and interventional respiratory physicians, taking into account the underlying and accompanying illnesses, as well as the patient's prognosis.

There are no noteworthy studies available that address the question of whether a routine bronchoscopy is indicated before or directly after decannulation. However, this guideline draws on expert opinion to recommend the use of endoscopy after decannulation (see below).

A follow-up bronchoscopy is absolutely essential if spontaneous closure of the tracheostoma is not completed by post-decannulation day 14 . In this instance, tracheal stenosis may have developed above the tracheostoma, which prevents spontaneous closure by increasing distal airway pressure with subsequent air leakage through the tracheostoma.

E22: An endoscopic control examination is recommended after the insertion of a tracheostomy retainer.

E23: Following decannulation, an endoscopic examination is recommended to ascertain the potential existence of a tracheal stenosis (even when not suspected).

\subsection{NIV and High-Flow-Oxygen Administration in}

Difficult-to-Wean Patients and the Post-Extubation

Phase

The topic of "NIV in difficult-to-wean patients and the post-extubation phase" is discussed in the corresponding section of the S3 Guideline, "Non-invasive Mechanical Ventilation in Acute Respiratory Failure" [16]. It should be mentioned, however, that this particular guideline relates to the use of NIV after one to several days of invasive MV (i.e., Group 2 corresponds to the definition by the International Consensus Conference [17]). Nonetheless, based on clinical experience, the recommendations can essentially be applied by analogy to a prolonged weaning situation. Since no high-quality scientific studies on the role of NIV in the prolonged weaning process exist, the recommendations expressed in the current guideline are based on expert clinical experience.

\subsubsection{Criteria for NIV Capacity during the Weaning}

Process

In the context of weaning prediction, the classic extubation criteria are generally used in addition to clinical assessment to estimate the ability of ventilated patients to adequately breathe spontaneously after extubation [175] (see Table 5 in Chapter 5.1.1).

These conventional predictors of weaning are at best useful as a guide for implementing NIV following invasive ventilation, because MV has to be continued due to ongoing respiratory insufficiency, and the only aspect that changes is the ventilation interface. An additional important prerequisite for NIV capacity subsequent to invasive $\mathrm{MV}$ is the ability of the patient to cooperate.

If it is considered to continue MV in the form of NIV directly after extubation, it is critical to check first whether the most important requirements for this are in place. The following conditions should be noted as absolute contraindications for the use of NIV [16]:

- lack of spontaneous breathing, presence of gasping

- fixed or functional displacement of the airways

- gastrointestinal bleeding or bowel obstruction

- dysphagia-related risk of aspiration

5.5.1.1. The Use of NIV following Hypercapnic Acute Respiratory Failure (ARF). If the NIV capacity of a patient is granted after invasive long-term MV, patients with hypercapnic chronic respiratory failure (CRF; e.g. after the exacerbation of severe COPD) should be extubated or decannulated in tracheotomy patient cases and transferred to NIV. However, based on the clinical situation, it should be foreseeable that no indication exists for obligatory ventilation that has a daily NIV requirement of $>12$ hours (up to 16 hours in individual cases). A high dependency on daily NIV would lead to a markedly restricted quality of life. In this context, it is necessary to clarify with the patient what his/her daily living situation is and what sort of demands exist from his/her side for the continuation of invasive or non-invasive MV. For a discussion of the ethics associated with this topic, please see Chapter 7.

Besides improving the weaning success rate and reducing the rates of mortality, reintubation, tracheotomy and complications following invasive ventilation [28, 439-441], NIV has also been successfully implemented in difficult-to-wean patients with neuromuscular diseases [442, 443] and diaphragmatic paralysis [444].

R24: If NIV capacity is granted after long-term invasive ventilation, extubation/decannulation and transfer to NIV is suggested for patients with hypercapnic ARF, if the clinical situation makes it foreseeable that no indica- 
tion for continuous, long-term ventilatory dependency exists.

5.5.1.2. The Use of NIV following Hypoxemic Acute Respiratory Failure (ARF). Analogous to the recommendations for NIV in ARF [16], NIV cannot be generally recommended for patients with hypoxemic acute respiratory failure who are undergoing prolonged weaning.

5.5.1.3. The Use of NIV in CRF following Weaning from Long-Term Invasive $M V$. In patients with advanced CRF - that is, those with signs of ongoing hypercapnia during continuous spontaneous breathing - it is necessary to check whether HMV is indicated once successful weaning has been confirmed. According to the definition established in Chapter 3, this corresponds to patients in Group 3b. Even though the data on this subject have so far relied on observational studies, up to $30 \%$ of successfully-weaned patients continue to be effectively provided with NIV in the home environment $[12,445]$. In view of 2 studies, one of which reported that NIV treatment led to a reduction in re-hospitalisation of COPD patients with persistent hypercapnia following an acute exacerbation [446], and another that showed reduced mortality in chronic hypercapnic COPD patients treated with NIV (German Multicentre Study, [447]), there is now a clear indication for the use of NIV in hypercapnic COPD patients after prolonged weaning. According to the indications outlined in the S2 Guideline "Non-Invasive and Invasive Home Mechanical Ventilation for Treatment of Chronic Respiratory Failure - Update 2017" [3], other disease groups that benefit from HMV after prolonged weaning are obesity hypoventilation syndrome, thoracic restrictive diseases and neuromuscular diseases with symptomatic hypercapnia.

E25: If CRF persists after extubation/decannulation, home MV is suggested, even after successful weaning has officially been established.

\subsubsection{High-Flow Oxygen for the Prevention of}

Post-Extubation Failure and Its Use in Weaning

Oxygen therapy has been mainly used thus far for the treatment of ARF and CRF, but is also used for treating patients with shock, sepsis, trauma or heart failure.

Different means of oxygen administration have long been available to spontaneously-breathing patients with acute pulmonary failure.

Over the last few years, high-flow oxygen administration via a nasal cannula has been used as an alternative to either NIV or low-flow oxygen therapy via a face mask [448]. This type of application delivers warmed, humidified oxygen via a nasal cannula, with flow rates of 40-60 litres per minute, and is subjectively well tolerated by patients.

On one hand, high-flow oxygen administration generates a certain positive end-expiratory pressure, whilst on the other, the work of breathing is reduced through washout of $\mathrm{CO}_{2}$ and the associated reduction in dead space [448-450]. The reduction in the work of breathing by means of $\mathrm{CO}_{2}$ wash-out and a subsequent reduction in dead space has already been demonstrated in earlier studies of the transtracheal administration of oxygen [451453].

An apparent connection exists between dead space and prognosis in hypoxemic ARF. This was demonstrated in a previous study, whereby an increased dead space in severe hypoxia arising from ARDS is accompanied by a higher mortality rate [454].

In addition to retrospective and observational studies in patients with hypoxemia, including those on the topics of extubation, post-operative patients and immune-compromised patients, randomised multicentre studies comparing NIV with high-flow oxygen therapy in patients with acute non-hypercapnic, hypoxemic pulmonary failure have recently been published [455-459].

The collective findings of numerous meta-analyses [460-468] demonstrate that high-flow oxygen therapy can serve as an alternative to both NIV and conventional oxygen therapy in the treatment of mild to moderate-severity ARDS.

From a practical perspective, the significant advantage of high-flow oxygen therapy is that it is easily applied and well tolerated by patients.

In comparison to this, the adaptation of the awake patient to both the mask (or helmet) and the ventilation mode is more complicated when using NIV. Furthermore, prolonged NIV often leads to mask-induced pressure sores, even though such side effects can be avoided in many cases by selecting a suitable mask and potentially applying padding to its contact surfaces.

In contrast to hypoxemic respiratory failure, no studies on the role of high-flow oxygen therapy in prolonged weaning were able to be found that could assess its value in this context. Therefore, no further statement on this topic will be made in the current guideline.

\subsection{Prolonged Weaning Strategies}

\subsubsection{Adjunctive Measures}

Besides the ventilation techniques and dealing with various interfaces (masks, tracheal cannula etc.) a rehabilitation strategy with a series of adjunctive measures is paramount in prolonged weaning. By no means exhaus- 
Table 15. Adjunctive measures in prolonged weaning

\begin{tabular}{|c|c|c|}
\hline Issue & Therapeutic measures & Goal \\
\hline $\begin{array}{l}\text { Pleural effusions/ } \\
\text { overhydration }\end{array}$ & $\begin{array}{l}\text { Drainage and, if necessary, pharmacotherapeutic measures such as } \\
\text { treatment with diuretics }\end{array}$ & $\begin{array}{l}\text { Improvement in respiratory mechanics and gas } \\
\text { exchange }\end{array}$ \\
\hline $\begin{array}{l}\text { Influence of respiratory } \\
\text { drive }\end{array}$ & $\begin{array}{l}\text { Oxygen administration for gas exchange disorders (e.g., pulmonary } \\
\text { emphysema), administration of morphine }\end{array}$ & $\begin{array}{l}\text { Reduction of an inadequately increased } \\
\text { respiratory drive and minute ventilation }\end{array}$ \\
\hline Body position & $\begin{array}{l}\text { Placing the body in a position that relieves breathing, such as raising } \\
\text { the upper body }\end{array}$ & $\begin{array}{l}\text { Reduction in the work of breathing, unloading } \\
\text { the diaphragm (especially in obesity, } \\
\text { neuromuscular diseases and thoracic restriction) }\end{array}$ \\
\hline Malnutrition or catabolism & Administration of a calories rich, low carbohydrate $(35-40 \%)$ diet & Prevention of excessive $\mathrm{CO}_{2}$ production \\
\hline Delirium and anxiety status & $\begin{array}{l}\text { Administration of anti-psychotics and anxiolytics (e.g., clonidine, } \\
\text { haloperidol and benzodiazepines), maintenance of circadian rhythm, } \\
\text { relaxation techniques }\end{array}$ & $\begin{array}{l}\text { Normalisation of autonomic and } \\
\text { neurophysiological function }\end{array}$ \\
\hline $\begin{array}{l}\text { Immobility and muscular } \\
\text { deconditioning }\end{array}$ & Physiotherapeutic measures, respiratory muscle training & $\begin{array}{l}\text { Mobilisation, reconditioning of atrophied } \\
\text { muscles }\end{array}$ \\
\hline Insufficient cough & $\begin{array}{l}\text { Percussion } \\
\text { Vibration and oscillation } \\
\text { Autogenic drainage } \\
\text { Postural drainage } \\
\text { Technical aids for forced coughing, for example } \\
\text { Mechanical cough assist } \\
\text { Manually-assisted cough (thoracic compression) } \\
\text { Mini-tracheotomy }\end{array}$ & Improved secretion clearance \\
\hline
\end{tabular}

tive, the following table presents these important adjunctive therapy measures in detail (Table 15). Some of the pathological and therapeutic considerations related to the topic of high respiratory drive can be found in the ensuing chapter (see Chapter 5.6.1.1).

5.6.1.1. The Influence of Respiratory Drive.

5.6.1.1.1. Reduction in Respiratory Drive. In patients undergoing prolonged weaning, an elevated respiratory drive with an increase in the work of breathing can lead to overloading and possibly to continuation of weaning failure. In this case, a reduction in respiratory drive can be useful in consecutively influencing the work of breathing. The common causal reasons for an increase in respiratory drive - which not only include pain, anxiety and withdrawal symptoms, delirium or infection, but also a suboptimal quality of MV setup - need to be treated effectively (see Chapter 5.1.2). For example, hypermetabolism accompanied by increases in respiratory rate, tidal volume, oxygen consumption, $\mathrm{CO}_{2}$ production and basal metabolic rate after finishing sedation can be successfully treated with clonidine, thus facilitating the weaning process [469]. In oxygenation disorders such as those arising from COPD or emphysema, the respiratory drive or respiratory minute ventilation are reduced by the delivery of oxygen, which ultimately leads to the desired decrease in the work of breathing. In justified cases, the elevated respiratory drive can be dampened by the administration of opiates while closely monitoring the ventilation or blood gases. This was exemplified in a study of 14 difficult-to-wean patients with tachypnoea and rapid shallow breathing, which showed that the application of opiates can effectuate a reduction in inspiratory effort and an improvement in breathing pattern, without having a significant influence on oxygenation and sedation [470]. However, close monitoring is an absolute requirement. A consecutive increase in $\mathrm{PCO}_{2}$ that corresponds to mild "permissive hypercapnia" (with simultaneous metabolic compensation of acidosis by bicarbonate retention) can be accepted under these circumstances.

$R 26$ : If a primary increase in the work of breathing is evident during prolonged weaning that cannot be explained by respiratory or weaning failure, the exclusion of other causes of increased work of breathing, such as pain, stress, or the presence of delirium, is suggested.

R27: After ruling out other causes, the administration of opiates with the aim of reducing the work of breathing may be indicated.

5.6.1.1.2. Stimulated Respiratory Drive. Although reduced respiratory drive is an unlikely cause of weaning failure, it should be taken into consideration when no 
other reason can be found [471]. The influence of different stimulants has so far been investigated, [472-476], with positive results in some instances. Recently, the effect of acetazolamide, a carbonic anhydrase inhibitor, was used in a prospective randomised study in patients with COPD and metabolic alkalosis [477], with the aim of stimulating respiratory drive through a reduction in bicarbonate [478]. From of a total of 382 patients, 187 were included in the treatment group. No significant differences were found in relation to the duration of $\mathrm{MV}$, time on the ICU, weaning success or survival rate [477]. In summary, there is currently no sufficient evidence for the use of respiratory-stimulating medications in patients undergoing prolonged weaning.

$R 28$ : The use of medications that directly or indirectly stimulate respiratory drive is not recommended for reducing weaning time in patients undergoing prolonged weaning.

5.6.1.2. The Role of Transfusion in Prolonged Weaning. Patients in prolonged weaning often have haemoglobin values below the normal range. In intensive care patients, but especially in patients in prolonged weaning, anaemia is associated with a poor outcome [479-485]. Red blood cell transfusions are performed with the aim of improving oxygen availability and thereby tissue oxygenation [486, 487]. A transfusion trigger defines the range from which the positive effects of an increase in haemoglobin levels outweigh the risks of unfavourable effects associated with transfusions.

In large randomised controlled studies, including those on sepsis [488], peri-operative patients [489, 490] for myocardial infarction [491], and gastrointestinal bleeding [492], no disadvantages - or even advantages - of a restrictive (mostly around $\mathrm{Hb}<7 \mathrm{~g} / \mathrm{dL}$ ) vs. liberal (mostly $\mathrm{Hb}<9 \mathrm{~g} / \mathrm{dL}$ ) transfusion threshold could be demonstrated. Even though anaemia is an independent risk factor for both pre-operative as well as non-surgical patients with cardiac diseases, a transfusion with 30\% haematocrit did not even lead to a better outcome in older patients [483]. The potential disadvantages of blood transfusions amongst other topics include transfusion-associated infections, transient deterioration of gas exchange [493], increased rate of ventilator-associated pneumonia and transfusion-induced acute lung injury (TRALI) [494-498]. Accordingly, national and international guidelines generally recommend a preference for restrictive transfusion thresholds [162, 499503].

For patients with prolonged weaning and more marked anaemia, there are no controlled comparative studies that have investigated the effects and complications of a liberal vs. restrictive red blood cell transfusion.

During the phases of MV in prolonged weaning, gas exchange usually is sufficient so that tissue hypoxia does not occur. However, during spontaneous breathing phases this may change significantly. Since a combination of respiratory pump failure (see Chapter 4.3) and cardiac failure often occurs in patients in prolonged weaning, the occurrence of anaemia can result in an additional load onto the respiratory pump through the increase in cardiac output. Unloading the respiratory and cardiac pumps by offsetting anaemia can lead to a reduction in total oxygen consumption, since both organs have an elevated autochthonous oxygen demand during insufficiency [488].

In patients with advanced pulmonary disease (such as COPD) and severe anaemia, a blood transfusion was found to significantly decrease the elevated work of breathing compared to anaemic patients with oncological diseases but without lung disease [104], which can be part of a therapeutic strategy in individual cases in prolonged weaning. Further studies provide support for a less restrictive transfusion trigger in prolonged weaning. In a small case series of severely-anaemic patients in prolonged weaning, transfusions were found to be associated with successful weaning from the ventilator [504]. A retrospective study of 138 difficult-to wean patients showed that haemoglobin values of $8-10 \mathrm{~g} / \mathrm{dL}$ were associated with a better weaning outcome than values below $8 \mathrm{~g} / \mathrm{dL}$ [103]. On the other hand, patients with $\mathrm{Hb}>10 \mathrm{~g} / \mathrm{dL}$ were significantly associated with weaning failure. Moreover, the different $\mathrm{Hb}$ levels were not due to transfusions and hence rather reflected the underlying condition of the patient [103].

The indication for a red blood cell transfusion is based on the assessment of the overall clinical picture, and cannot be established by means of laboratory findings alone (haemoglobin, haematocrit, erythrocyte count), since inadequate tissue oxygenation can occur at different $\mathrm{Hb}$ concentrations $[487,505-510]$. The recommendation for the use of physiological transfusion triggers (Table 16) is made with the aim of enabling patients with limited compensatory capacity to deviate individually from the restrictive transfusion trigger, taking into account the overall clinical picture. On this note, it is important to mention that the current guideline pertains to patients in prolonged weaning in whom a markedly increased work of breathing obstructs their weaning success. This therefore counts as an additional physiological transfusion trigger in prolonged weaning. 
Table 16. Physiological transfusion triggers can be suggestive of anaemic hypoxia if anaemia is confirmed by laboratory chemistry and normovolaemia is maintained (adapted from [501])

Physiological transfusion trigger Cardiopulmonary symptoms
Tachycardia

Hypotension

Drop in blood pressure with unknown genesis

High work of breathing
Typical signs of ischemia in the ECG

Newly occurring regional myocardial contraction disorder in echocardiography Global indices for insufficient oxygen supply
Newly occurring ST depressions or elevations Newly occurring arrhythmia

\author{
Increase in global $\mathrm{O}_{2}$ extraction of $>50 \%$ \\ Reduction in $\mathrm{O}_{2}$ uptake of $>10 \%$ of the baseline value \\ Reduction in mixed venous $\mathrm{O}_{2}$ saturation of $<50 \%$ \\ Reduction in mixed venous $\mathrm{PaO}_{2}$ of $<32 \mathrm{~mm} \mathrm{Hg}$ \\ Reduction in central venous $\mathrm{O}_{2}$ saturation of $<60 \%$ \\ Lactate acidosis (lactate $>2 \mathrm{mmol} / \mathrm{L}+$ acidosis)
}

Further criteria to consider are as follows [501]:

- Cause, duration, and severity of anaemia

- Extent and speed of blood loss

- Estimation of individual physiological capacity to compensate for the minimised arterial $\mathrm{O}_{2}$ content

- Pre-existing illnesses in the patient, which limits compensation capacity in acute anaemia (e.g., cardiac, vascular, pulmonary illness)

- Current clinical status of the patient

- Symptoms that can indicate the presence of anaemic hypoxia (physiological transfusion trigger)

- Intravasal volume status, since an erythrocyte deficit is not reliably detected with diminished plasma volume (hypovolaemia), and high haematocrit values are measured (see acute blood loss).

The installation of a central venous catheter can be considered for additionally determining central venous oxygen saturation $\left(\mathrm{ScvO}_{2}\right)$. The $\mathrm{ScvO}_{2}$ can help evaluate the relationship between oxygen uptake and release, which in turn can establish whether a patient will benefit from a transfusion. This approach of using a differentiated indication is confirmed by more recent studies [506]. A threshold value of $\leq 65 \%$ was recently reported to be necessary for an improvement in $\mathrm{ScVO}_{2}$ in cardiac, thoracic and vascular surgery intensive care patients [511]. Experimental approaches with direct measurement of tissue oxygenation at the intracellular level (mitochondrial $\mathrm{PO}_{2}$ ) remain to be clinically confirmed [512].

A Hb level of $>8-10 \mathrm{~g} / \mathrm{dL}$ can be aimed for in patients with impaired compensation whose clinical symptoms (physiological transfusion trigger; see Table 16) are suggestive of anaemic hypoxia. In some cases, an elevation of the $\mathrm{Hb}$ value to $>10 \mathrm{~g} / \mathrm{dL}$ can be indicated, but are not essentially recommended.

R29: A target $\mathrm{Hb}$ value between 7 and $9 \mathrm{~g} / \mathrm{dL}$ (4.34$5.59 \mathrm{mmol} / \mathrm{L}$ ) is suggested for patients undergoing prolonged weaning from the ventilator, while an increase in the $\mathrm{Hb}$ value to $>10 \mathrm{~g} / \mathrm{dL}$ is not recommended.

R30: Consideration of physiological transfusion triggers is suggested when establishing the indication for a transfusion.

R31: Raising the $\mathrm{Hb}$ value $(\mathrm{Hb}>8 \mathrm{~g} / \mathrm{dL}$ ) is suggested for patients in prolonged weaning whose clinical symptoms indicate either inadequate compensation of low $\mathrm{Hb}$ levels, or that the markedly increased work of breathing (as in severe pulmonary disease) is impeding weaning success.

5.6.1.3. Improving Nutritional Status and Metabolism. Both mal - and overnutrition can prolong the weaning phase. Patients in prolonged weaning are, however, often already initially malnourished due to pre-existing pulmonary disease (pulmonary cachexia) or become malnourished during the critical phase of illness through inadequate calorie and protein intake that results from severe catabolism [513, 514]. Therefore, malnutrition is also common in patients with chronic critical illness and obesity. Even in parenteral form, targeted nutritional therapy has a beneficial effect on total body protein, muscle strength and respiratory function, especially in cases of a nutritional deficit [515]. In critically-ill patients, however, protein loss cannot be prevented through a positive, non-protein energy balance alone [516]. A complete parenteral nourishment increases $\mathrm{CO}_{2}$ production $\left(\mathrm{VCO}_{2}\right)$ in ventilated patients via glucose supply up to the point of 
hypercapnic acidosis [517]. This can lead to an additional and avoidable increase in the work of breathing, especially in the prolonged weaning phase, and speaks for enteral nutrition $[518,519]$.

In a double-blinded prospective randomised controlled trial (PRCT; $n=20$ ) al-Saady et al. [520] showed that patients in prolonged weaning who received a highfat, low-carbohydrate enteral nourishment ("high-fat/ low-carb") had a significantly reduced MV time compared to patients in the control group. In another PRCT, $(n=32)$, van den Berg et al [521] found that such a fat-rich enteral diet significantly reduces $\mathrm{CO}_{2}$ release in weaning patients, whereas no change in $\mathrm{PaCO}_{2}$ was observed. However, according to an evaluation of these studies by the Guideline Committee of both the Society Critical Care (SCCM) and the American Society for Parenteral and Enteral Nutrition (ASPEN) 2016 [518], these effects should not be overestimated in macronutrient composition, provided that energy supply does not exceed energy demand [522].

If swallowing is impaired, there is a risk that the aspiration tendency will increase by using a nasogastric tube as an access point for enteral feeding. To this end, if artificial enteral feeding is expected to exceed more than 3-6 weeks, an early indication for a PEG (percutaneous endoscopic gastrostomy) feeding tube can be established [523, 524]. In patients who were on MV for more than 5 days, measurements over the first 7 days following extubation revealed that the maximum oral calorie intake only comprised $50 \%$ of the daily requirement [525]. In the anabolic phase of rehabilitation that follows the weaning period, energy intake should preferably amount to 1.2-1.5 times the calculated energy requirement, although the corresponding controlled studies are lacking [524, 526]. For these difficult-to-treat patients, the use of indirect calorimetry can be helpful in accurately determining energy requirements [524, 527, 528]. Oral nutritional supplements (sip-feed supplements), as well as the continuation of tube feeding, especially come into consideration for patients whose calorie intake requirements cannot be met by oral feeding alone [528]. In these instances, the duration of supplementation is based on the nutritional status of the patient. For an approximation of the basal energy requirement, and as recommended by the guidelines of the German Society for Nutritional Medicine (DGEM) [524], the energy requirement in non-obese intensive care patients $\left(\mathrm{BMI}<30 \mathrm{~kg} / \mathrm{m}^{2}\right)$ can be estimated at 24 $\mathrm{kcal} / \mathrm{kg}$ of the current body weight/day.

Numerous controlled studies have investigated the effects of an enterally-administered diet enriched with omega-3 fatty acids (eicosapentaenoic acid, EPA) and gamma linolenic acid (GLA), in patients with pulmonary failure, lung injury and/or sepsis [529-532]. Although none of these studies evaluated the weaning phase separately, they did show that patients who received the supplementary nutrition had a significantly shortened duration of MV and stay in the ICU [532], a significantly more favourable Horowitz index value [530] and a significantly higher survival rate in sepsis [532]. In two meta-analyses, the administration of EPA and GLA was confirmed to be advantageous for significantly lowering the mortality rate [532], for oxygenation, and for prolonging the time without ventilatory support [533]. In contrast, a double-blind, placebo-controlled multicentre study reported conflicting results on this topic [534]. The study recruited 272 patients who had developed lung injury within the previous 48 hours, and were treated across 44 hospitals in the US National Heart, Lung, and Blood Institute ARDS Clinical Trials Network. An enteral supplement with omega- 3 fatty acids, GLA and antioxidants was compared to an isocaloric control solution, each of which was administered twice a day and delivered separately from the enteral nutrition. The number of ventilator-free days served as the primary end-point of the study. Plasma ELA levels increased significantly in the intervention group. The study was stopped prematurely, since the patients in the intervention group spent significantly more time than the control group on the ventilator (14.0 vs. 17.2 days; $p=0.02)$ and in the ICU (14. 0 vs. 16.7 days; $p=0.04$ ). The 60 -day mortality rate was not significantly increased in the omega-3 group, although these patients had significantly more diarrhoea. Based on these findings, the authors concluded that the administration of an omega-3 supplement in this group of patients does not improve treatment outcome, but may actually impair it. However, a point of criticism is that the hypocaloric nutrition in each group only contained 400 and 800 calories, respectively; therefore, the negative effects could also be the result of an insufficiently low protein intake. Two meta-analyses of 6 [535] and 7 [536] controlled studies did not identify any clear clinical advantages of an omega-3 fatty acid-enriched diet for patients with lung injury/ ARDS. Due to the controversial data on this topic, the 2016 SCCM/ASPEN Guidelines do not provide any recommendation for the administration of omega-3 fatty acid-enriched enteral nutrition [518]. The current DGEM Guideline does not recommend it [524], while in the current ESPEN Guideline, the recommendation is only valid for high-dose applications [528]. 
Table 17. Causes of secretion retention in prolonged weaning

Increased secretion formation in the airways
Elevated MUC-gene production [539], which, for example, can occur during infection or through irritation of the airway mucosa by foreign objects such as a tracheal cannula

Proliferation of goblet cells and/or submucosal glands in chronic inflammatory airway diseases or acute exacerbation of chronic pulmonary diseases

Suctioning injuries by suctioning too deeply in the endobronchial rather than endotracheal region

\section{Congenital ciliary dysfunction}

Replacement of cilia-lined epithelium with another type, such as squamous epithelium (so-called squamous cell metaplasia)

Disruption to ciliary function by an endotracheal or tracheal cannula; excessive inspiratory oxygen concentration or insufficient conditioning of the respiratory gases Inspiratory and/or expiratory muscle weakness, for example, in ICUAW (ICU aquired weakness) or other neurological diseases

Lack of effective glottis closure, for example, in the presence of an endotracheal tube or a blocked tracheal cannula

Unstable thorax or inadequately treated pain after thoracotomy/sternotomy

Aspiration of saliva/food See Chapter 5.6.1.5

Table 18. Consequences of secretion retention in prolonged weaning

- Increased work of breathing through the narrowing of airway diameter or reduced lung compliance caused by atelectasis

- Worsening of blood gases due to ventilation-perfusion mismatch up to the shunt caused by atelectasis

- Increased risk of infection

- NIV failure after decannulation

Both the SCCM/ASPEN guidelines as well as the recommendations by an international expert summit for intensive care patients rather emphasise the administration of a hypocaloric (80-90\%), but highly protein-rich diet at a dosage of $1.2-2.5 \mathrm{~g} / \mathrm{kg}$ body weight [537], preferably in combination with muscle training [538]. The current DGEM guidelines recommend an individualised protein dose of $1.0 \mathrm{~g} / \mathrm{kg}$ body weight/day, or $1.2 \mathrm{~g} / \mathrm{kg}$ body weight/day amino acids [524].

R32: Artificial feeding that is preferably oral or enteral is suggested for patients undergoing prolonged weaning. If artificial enteral nutrition is expected to exceed 6 weeks, establishing an early indication for a PEG or PEJ tube is suggested.

R33: An energy intake that does not exceed energy demand is suggested for patients in the weaning phase. A sufficiently high intake of protein $(1.0 \mathrm{~g} / \mathrm{kg}$ body weight/ day or $1.2 \mathrm{~g} / \mathrm{kg}$ body weight/day amino acids) as well as the intake of electrolytes, vitamins and trace elements should be ensured.

5.6.1.4. Secretion Management. Secretion retention in the airways presents as a particular challenge, especially in prolonged weaning; nonetheless, literature that specifically relates to secretion management in this patient group is currently lacking. The available literature covers physiotherapy-based studies with short term analyses and expert recommendations that are based on the physiological mechanisms of secretolysis and expectoration.

Despite the lack of evidence, this guideline affords secretion management an important role in prolonged weaning, which always needs to be adapted individually, depending the secretion amount and cause of retention.

The different factors that are responsible for the accumulation of secretions in the deep respiratory airways are presented in Table 17. The consequences of such retention are presented in Table 18.

There are two different methods for improving secretion clearance in prolonged weaning: measures for secretion lysis or mobilisation, and measures for improving secretion removal from the airways. Clear identification of the cause of secretion accumulation (e.g. increased secretion formation, specific secretion properties such as high viscosity, weak expectoration) is important for the efficient application of the different secretion management techniques.
Schönhofer et al. 
Table 19. Physiological mechanisms for the treatment of secretion retention (according to [240])

- Increase in intrathoracic volume

- Reinforcement of the maximal expiratory flow

- Secretolysis, for example, oscillation therapy

- Increase in expiratory volume

- Endotracheal suctioning

In terms of the underlying physiology, there are 5 principles, in addition to pharmacotherapy (see Table 19), by which secretion elimination from the airways can be achieved [240].

When determining the indication for accompanying pharmacotherapy, it is important to consider the variable composition of the bronchial secretion based on the respective underlying disease, such as when using recombinant human DNAse [540].

For medication-based secretolysis, the AARC (American Association for Respiratory Care) Clinical Practice Guideline [541] states the following:

- rhDNA should not be used in patients without underlying cystic fibrosis

- Routine bronchospasmolytic therapy and the administration of $\mathrm{N}$-acetylcysteine to aid in secretion clearance are not recommended

Apart from invasive techniques such as bronchoscopy and endotracheal suctioning (carried out by nursing staff or respiratory therapists, or by physiotherapists and speech therapists, where approved), physiotherapy holds an important place in the weaning process by restoring various functions and hence supporting the rehabilitation process. The main focus is on patient mobilisation and the associated measures such as passive and active respiratory therapy, which includes secretion management. It is particularly important that despite penetration or aspiration through decannulation in mild to moderate dysphagia, the use of an effective secretion management process can prevent the patient from having to be transferred to an outpatient facility with a tracheostoma (Group 3a II according the revised Weaning Classification, Table 3). This requires close collaboration between physiotherapists, respiratory therapists and speech therapists. Besides respiratory therapy, special techniques for secretion lysis [542] are also deployed in the secretion management process, such as those using endobronchial or transthoracic oscillatory systems [543-545], or inhalative therapy such as hypertonic saline solution [546]. Secretion elimination can subsequently take place via posi- tioning therapy, huffing, a PEP system [547, 548], manually-assisted cough [549] or use of mechanical coughing aids such as the mechanical insufflator-exsufflator [550552], which is only indicated for patients with neuromuscular diseases and healthy lungs. However, according to a Cochrane analysis, a limitation of the latter technique is that there is no compelling evidence for the use of mechanical coughing aids in some patient groups including those undergoing weaning from the ventilator [553]. However, this proof-of-concept is essentially lacking, owing to the heterogeneity of the study patients; however the use of mechanical coughing aids has few side effects.

All of the physiotherapeutic measures that are taken to promote secretion clearance are based on the principle of first getting air behind the secretion, then transporting it into the central airways by increasing intrathoracic pressure either during the expiratory phase or coughing. For example, in cases of bronchial instability that co-exists with COPD, the use of positive pressure during expiration can lead to shifting of the so-called equal pressure points to the large airways, thus improving secretion clearance. Depending on the underlying pathophysiology of the secretion retention, and following detailed examination, an individually-tailored physiotherapy program is necessary, such as the one presented schematically in Figure 7, which is based on the recommendations by the ERS and ESICM Task Force [240].

In cases of weak cough, a mini-tracheostomy is an alternative to a tracheostomy retainer after decannulation. Due to the relatively thin calibre of the tube (inner diameter: $4.0 \mathrm{~mm}$ plus wall thickness), it allows ongoing shrinkage of the tracheostoma, and aspiration of secretions from the trachea via a suctioning catheter. This especially pertains to patients with neuromuscular diseases who require NIV directly after decannulation, but have a weak cough. Thus, the mini-tracheotomy enables the simultaneous application of NIV with effective secretion management.

Further details on the practical aspects of secretion management, especially in relation to the treatment of patients with neuromuscular diseases and COPD, can be found in the secretion management chapter of the S2k "Guidelines for Non-Invasive and Invasive Home Mechanical Ventilation for Treatment of Chronic Respiratory Failure" [3].

As described for mobilisation, is it absolutely essential that secretion lysis and removal is also regularly performed 7 days a week, and if necessary, several times a day. 


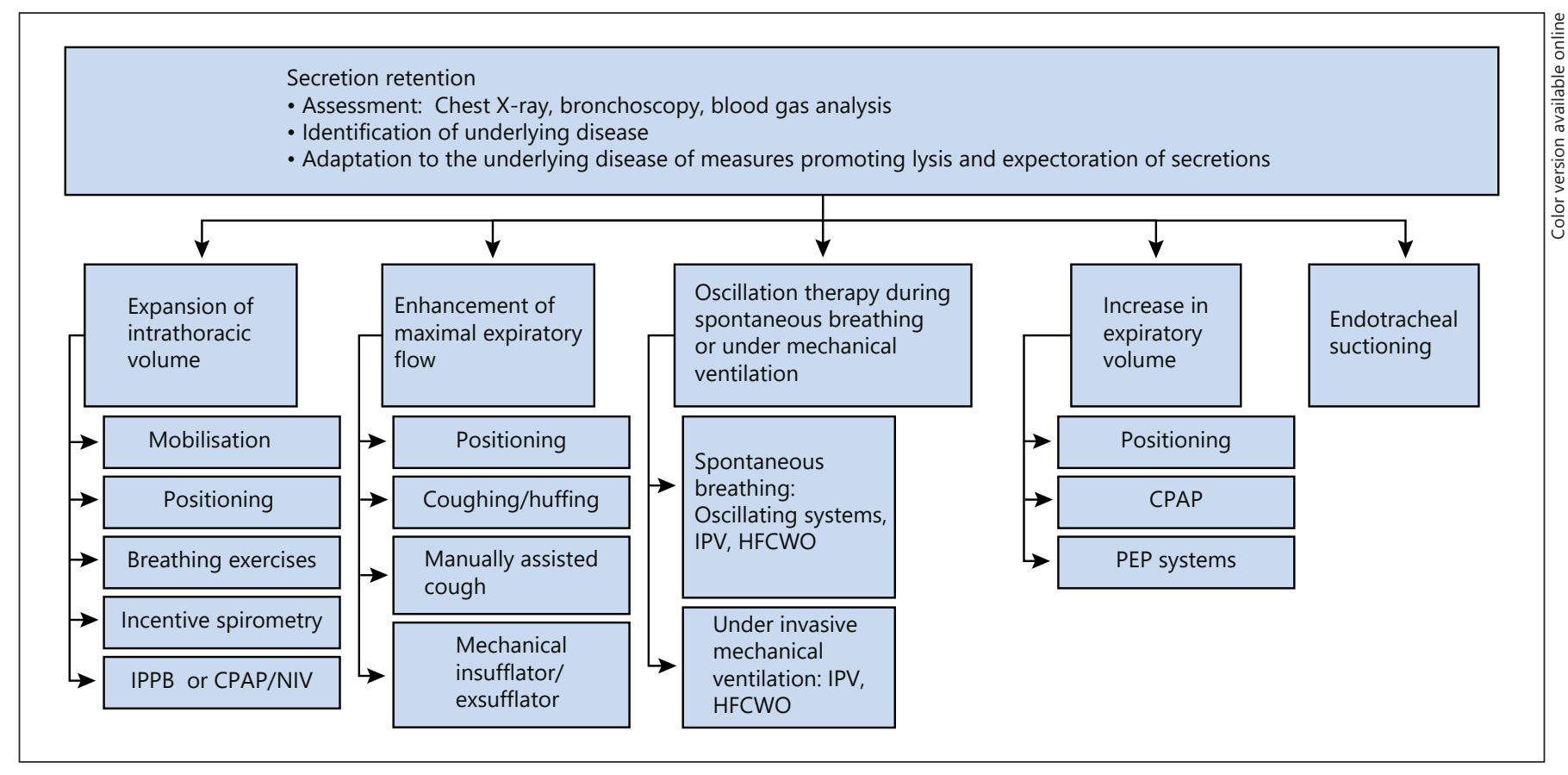

Fig. 7. Measures for secretion lysis and removal in prolonged weaning [240]. HFCWO, high frequency chest wall oscillation; IPPB, intermittent positive pressure breathing; CPAP, continuous positive airway pressure; NIV, non-invasive ventilation; PEP, positive expiratory pressure; IPV, intrapulmonary percussion ventilation [rerif].

Table 20. Possible causes of dysphagia during the weaning process

- Presence of dysphagia prior to MV and weaning (e.g., cerebral ischemia, traumatic brain injury, neuromuscular diseases)

- Longer phases of MV with neurological disease [554]

- ICUAW (critical-illness polyneuropathy/myopathy)

- Reduced state of consciousness (underlying disease, side-effect of sedatives)

- Endotracheal intubation [555-557]

- Tracheal cannula - prevention of laryngeal elevation during swallowing

- Swelling/injury in the hypopharyngeal/laryngeal region, for example, due to nasogastric tube

- Deprived swallowing/coughing reflex of the larynx through reduced stimulation of sensory receptors due to a chronically blocked artificial airway [558]

- Sepsis [559]

R34: Due to its central role in prolonged weaning, secretion management is recommended to be carried out on a daily basis, including at weekends.

R35: In patients with neuromuscular diseases and NIV who otherwise have treatment-resistant hypersecretion, a mini-tracheotomy can be helpful for effective secretion removal.
QI2: During the weaning process, secretion management is carried out daily, including weekends (number of patients that had secretion management/number of patients that should have had secretion management).

5.6.1.5. Management of Dysphagia. Dysphagia with subsequent aspiration often presents as a cause for failed decannulation or recurring infections of the lower airways in patients undergoing prolonged weaning. A list of the multiple causes of dysphagia is presented in Table 20.

Whether the tracheal cannula itself serves as an independent risk factor remains under debate. Some studies negate this $[560,561]$, especially since the larynx can be adequately lifted during swallowing with an existing tracheal cannula [562]. On the other hand, in a prospective study in patients who underwent dilatative tracheotomy after a ventilation duration of $20 \pm 11$ days, Romero et al. used FEES (fiberoptic evaluation of swallowing) to identify dysphagia in $38 \%$ of the patients [374]. It is clear that even with an inflated tracheal cannula, aspiration cannot be completely prevented, since pressures exceeding that of the cuff may result during swallowing [563].

Particularly for neurological patients in prolonged weaning, it is important that the possibility of dysphagia is always considered and that is it ruled out or confirmed, 
Table 21. Penetration-aspiration scale (PAS) by Rosenbek et al. [569]

\begin{tabular}{ll}
\hline $\begin{array}{l}\text { Classi- } \\
\text { fication }\end{array}$ & Characteristics \\
\hline 1 & No penetration \\
\hline 2 & $\begin{array}{l}\text { Laryngeal penetration above the vocal cords, complete } \\
\text { ejection }\end{array}$ \\
\hline 3 & $\begin{array}{l}\text { Laryngeal penetration above the vocal cords, no } \\
\text { ejection }\end{array}$ \\
\hline 4 & $\begin{array}{l}\text { Laryngeal penetration up to the vocal cords, complete } \\
\text { ejection }\end{array}$ \\
\hline 6 & $\begin{array}{l}\text { Laryngeal penetration up to the vocal cords, no } \\
\text { ejection }\end{array}$ \\
\hline 7 & $\begin{array}{l}\text { Aspiration, with ejection from the trachea (coughing of } \\
\text { aspirate into the laryngeal inlet and beyond) }\end{array}$ \\
\hline 8 & $\begin{array}{l}\text { Aspiration, no ejection from the trachea with weak } \\
\text { coughing }\end{array}$ \\
\hline
\end{tabular}

not only by clinical examination, but also with the aid of FEES. Speech therapy expertise should be incorporated into the management of dysphagia, not only for diagnosis but also for treatment.

There are no prospective studies that address the frequency of dysphagia occurrence in prolonged weaning and its influence on the course of weaning. In a patient cohort with an a priori higher probability of developing a swallowing disorder due to the underlying disease, dysphagia was described in up to $87 \%$ of the patients admitted to early neurological rehabilitation program [564].

Due to the high prevalence of swallowing disorders, an evaluation of the upper respiratory tract with the specific question of whether dysphagia exists should be routinely performed in the weaning process.

An aspiration tendency can often be clinically proven, for example, by aspirate suctioning, but can also be identified by staining the saliva or food, for example, with food colouring when the tracheal tube is deflated. Flexible endoscopy through the existing tracheal cannula allows the visualisation of injuries such as arytenoid cartilage dislocations and mucous membrane swelling in the laryngeal inlet, as well as a salivary pool in the larynx opening, as a manifestation of dysphagia. Simultaneous examination of laryngeal sensitivity is also possible in awake patients.
Two additional procedures exist for co-operative, alert patients:

(a) Modified Evan's Blue Dye-Test [565]: After staining the saliva or food, colour that appears around the inflated cannula coming out of the tracheostoma is a reliable indicator of aspiration; coloured aspirate can be additionally suctioned when the cannula is deflated. This test has a high predictive value for aspiration [566]; however, this method is limited by the fact that it cannot be used to diagnose penetrations, or quantify the degree of dysphagia.

(b) Endoscopic transnasal evaluation of swallowing (FEES): This is seen as the gold standard in swallowing diagnostics [567], and not just in adult patients [568]. A bronchoscope (ideally with video attachment) is led into the meso/hypopharynx via the right or left nostril, without local anaesthetic. This enables the investigator to determine whether there is an accumulation of saliva in the hypopharynx, and also allows the assessment of the swallowing of food that is dyed and of different consistencies (liquid, mushy, solid, crumbly). This means that the passage of food into the laryngeal inlet (penetration) and through the vocal cords (aspiration) can be reliably detected. This method also allows detection of residues in the hypopharyngeal area that are often a sign of disrupted sensitivity and can be secondarily aspirated with a change in body position. The penetration-aspiration scale devised by Rosenbek et al. [569] (see Table 21) has emerged as the most appropriate test for grading the severity of the swallowing disorder, and especially for the assessment and comparison of sequential examinations.

The approach to dysphagia management in early neurological rehabilitation patients is described in the relevant specialised literature [570].

The procedures described above are complementary and provide more detailed information about the oral, pharyngeal and oesophageal phases of swallowing. Based on the findings of the diagnostic methods with which the treating team has the most experience, a problem-oriented and individual strategy is then developed, and should also include close consultation with a speech therapist. For large pools of saliva above the larynx, anti-cholinergics (e.g., scopolamine patch) or individual treatment with botulinum toxin injections in the salivary glands [571] can be used to reduce salivary production. This allows the tracheal cannula to be deflated more easily, increasing physiological airflow and improving proprioception in the upper airways. As a general rule in patients who are fitted with an blocked tracheal cannula, it is important to use systems that allow subglottic suctioning [563]. 
Specific dysphagia treatment has the goal of avoiding aspiration and enabling oral intake of food. This therapy should always be carried out on an interdisciplinary basis; amongst other things, logopaedic measures after longterm intubation or tracheostomy are available in prolonged weaning. Swallowing exercises and attempts under the guidance of speech, occupational and/or respiratory therapists promote the restoration of the normal motoric component of swallowing and the learning of compensatory swallowing techniques. Isolated penetrations do not represent a contraindication for oral food intake, and even aspirations with self-cleaning of the larynx can be tolerated. Deflating the cuff under spontaneous breathing conditions in combination with a speaking value to promote coughing can help with this process to some extent [572]. For definitive decannulation to occur, aspiration should be specifically ruled out, for example, with FEES. For reasons of quality-of-life and improved communication, decannulation can take place earlier if the patient has been explicitly informed about the risk of aspiration and its consequences, especially when the actual or presumed will of the patient does not consider the primary therapy goal as fulfilling his/her life span.

$R 36$ : Prior to beginning oral food intake after invasive $\mathrm{MV}$, an early test for the presence of dysphagia is recommended.

R37: If dysphagia is present, regular swallowing training under the guidance of a speech therapist is recommended.

R38: Prior to decannulation, clinical and if possible technically assisted exclusion of aspiration is recommended.

QI3: Screening for Dysphagia: Number of patients in prolonged weaning in whom dysphagia screening was performed prior to starting oral nutrition/Number of patients in prolonged weaning with oral nutrition

5.6.1.6. Measures for Improving Diaphragm Function in Prolonged Weaning.

5.6.1.6.1. Inspiratory Muscle Training (IMT). It is possible to carry out IMT in patients with diaphragmatic weakness using specific techniques.

Of the available methods for IMT, three recognised techniques can be applied in patients with diaphragmatic weakness: (1) inspiratory threshold loading, (2) targeted resistive breathing, and (3) normocapnic hyperpnea [573].

Both a systematic review [574] and a randomised controlled study [575] related to the topic of inspiratory muscle training were recently published.
The review analyses ten studies and comes to the conclusion that conducting inspiratory muscle training in a selected patient cohort at varying degrees of effectiveness can reduce the duration of the weaning process and shorten the time required on NIV after extubation [574]. The randomised study investigated the effect of IMT after successful weaning [575]. The training led to an increase in muscle strength and quality of life, whereas an effect on hospital mortality rate was not found. Based on the results of this study, future research should investigate whether IMT in selected patient groups confers a reduction in the rate of post-extubation failure.

IMT favourably influences both respiratory muscle function and weaning success in invasively ventilated patients undergoing the weaning process $[311,576]$, and can be used without endangering patients, provided they are appropriately selected [575]. Whether a protective anti-VIDD effect can be achieved here currently remains open, despite indirect evidence such as a reduced rate of post-operative pulmonary complications and a reduced hospital stay in high-risk patients prior to arteriovenous bypass surgery [577].

5.6.1.6.2. Respiratory Muscle Support by Indirect and Direct Stimulation of the Diaphragm. The surgical implantation of devices to stimulate the phrenic nerve was already described in the 1940's, and has since been implemented in the treatment of spinal cord injury $[578,579]$. A significant cause of weaning failure is insufficient diaphragmatic strength or diaphragmatic dysfunction. A relatively new approach that is potentially therapeutic is the temporary stimulation of the diaphragm to support respiratory muscle training. This serves to expedite regeneration of the diaphragm and hence weaning from ventilation. The first clinical data have been published for diaphragmatically implanted probes, as well as for transvenous catheters, which trigger diaphragmatic contraction by stimulating the phrenic nerve [580-584]. In animal experiments it could be shown that pure controlled ventilation leads to significant diaphragmatic atrophy and that this can be reduced by intermittent stimulation of the phrenic nerve [582].

In a clinical study of 22 patients who had undergone elective surgery (for correction of an atrial septal defect), the basic applicability of the method as well as an effective triggering of diaphragmatic concentration was shown, thus demonstrating the general viability of this technique in the clinical setting [583].

A prospective randomised controlled clinical study is currently being carried out in Europe (Percutaneous temporary placement of a phrenic nerve stimulator for dia-
Schönhofer et al. 
phragm pacing [RESCUE2]) [584]. The study encompasses 88 adult patients $(n=88)$ with a ventilation duration of $>96 \mathrm{~h}$, and in whom at least two weaning attempts were not successful.

Whether the effects of temporary diaphragmatic stimulation ultimately appear to justify the use of this invasive method cannot yet be definitely ascertained.

The results of ongoing and future prospective clinical studies are still awaited, especially those pertaining to possible complications. Due to the limited data availability, it is not possible to definitively assess the value of this procedure.

\subsubsection{Extracorporeal Lung Support Procedures}

In an extracorporeal lung support (ECLS) procedure, blood is pumped through a membrane oxygenator (usually) via a venous cannula and through a tube system for saturation with oxygen and elimination of carbon dioxide, and is then returned to the circulation. Carbon dioxide elimination can alternatively be performed using a special dialysis procedure, such as bicarbonate elimination. The important determinants of the effectivity of an ECLS procedure are the blood/pump flow or fresh gas flow over the gas exchange membrane. Depending on the system and the diameter of the cannula, a blood flow rate of up to $5 \mathrm{~L} / \mathrm{min}$ is possible. Whereas sufficient blood flow through the oxygenator (generally $>1.5 \mathrm{~L} / \mathrm{min}$ ) is required for efficient oxygenation of the blood, efficient $\mathrm{CO}_{2}$ elimination requires lower flow rates, which are decisively defined by the extent of fresh gas flow $[585,586]$. The potential indications and areas of application for ECLS procedures theoretically encompass nearly all forms of acute and chronic lung failure, and are dependent on the efficiency and invasiveness of the systems.

Two different types of systems are currently available: - High-Flow Systems enable extracorporeal membrane oxygenation (ECMO) and primarily come into use in patients with severe lung failure, when all other conservative measures have been exhausted and adequate oxygenation cannot be guaranteed. According to the S3 Guideline "Invasive Mechanical Ventilation and Extracorporeal Membrane Oxygenation in Acute Respiratory Insufficiency", their use is linked to structural characteristics and should take place in a centre with expertise in the treatment of these patients [162].

- Low-Flow Systems do not have any significant effect on oxygenation due to the markedly lower extracorporeal blood flow. They are almost exclusively limited to carrying out extracorporeal $\mathrm{CO}_{2}$ removal $\left(\mathrm{ECCO}_{2} \mathrm{R}\right)$ and have found their place mainly in patients with acute hypercapnic respiratory failure. Extracorporeal $\mathrm{CO}_{2}$ removal can either occur via a pumpless arteriovenous ( $\mathrm{pECLA}$ ) or a pump-driven veno-venous extracorporeal procedure. Systems in which kidney support procedures are combined with $\mathrm{ECCO}_{2} \mathrm{R}$ by a membrane lung, or where $\mathrm{CO}_{2}$ removal takes place via bicarbonate ultrafiltration or electrodialysis also fall into this category [587-590].

During $\mathrm{ECCO}_{2} \mathrm{R}$, depending on how high the amount of extracorporeally-eliminated $\mathrm{CO}_{2}$ is in relation to the amount of $\mathrm{CO}_{2}$ produced by the patient, a part or the entire work of breathing required for $\mathrm{CO}_{2}$ removal can be taken over by $\mathrm{ECCO}_{2} \mathrm{R}$. According to a series of prospective and retrospective case-control studies, a significant reduction in $\mathrm{PaCO}_{2}$ and an increase in $\mathrm{pH}$ can be achieved with this process [591-595]. In patients in prolonged weaning, it therefore appears theoretically possible that the use of $\mathrm{ECCO}_{2} \mathrm{R}$ can facilitate weaning from the ventilator or replace the ventilator. The significant potential advantages of $\mathrm{ECCO}_{2} \mathrm{R}$ in comparison to prolonged $\mathrm{MV}$ are the avoidance of ventilator-associated complications and the increased level of comfort for the patient. Nonetheless, these potential advantages need to be weighed up against the complications associated with the $\mathrm{ECCO}_{2} \mathrm{R}$ procedure (e.g., blood vessel injury, requirement for therapeutic anti-coagulation, bleeding and thrombo-embolic events).

There are no prospective randomised clinical studies available in which the effects of $\mathrm{ECCO}_{2} \mathrm{R}$-based weaning versus conventional weaning were investigated in patients undergoing prolonged weaning. Since the $\mathrm{ECCO}_{2} \mathrm{R}$ procedure has already been in use for a number of years in Germany [596], it should first be investigated in controlled prospective studies. At this point in time, it is considered to be an experimental procedure. Therefore, based on the available data, a recommendation for or against the use of $\mathrm{ECCO}_{2} \mathrm{R}$ in prolonged weaning cannot yet be made.

\subsubsection{Sleep on the ICU}

Both sleep and circadian rhythm can be substantially disrupted in some patients during their stay on the ICU - various studies report the occurrence of frequent episodes of arousal, loss of deep/REM sleep and an abnormal distribution of sleep and awake periods, even during the day [597-600]. Sleep deprivation has a negative effect on the immune system, the cardiovascular system and cognitive capacity, and respiratory muscle strength [601]; it is also a risk factor for the development of delirium and 
can increase the duration of time required on the ICU as well as the mortality rate.

Huttmann et al. [602] investigated sleep quality in 19 tracheotomised ICU patients undergoing weaning using polysomnography and questionnaires. They found that sleep quality was reduced, albeit with the preservation of high amounts of slow-wave sleep, and that the arousal index was $18.7 \pm 12.4 / \mathrm{h}$. No differences were found between successfully and unsuccessfully weaned patients, while increased bicarbonate levels negatively correlated with sleep efficiency and quality.

The following factors can be responsible for a disruption to sleep architecture:

- Noisy surroundings (especially that caused by nursing staff)

- Mechanical ventilation

- Inflammation

- Inadequate treatment of pain

- Effects and side-effects of medication

- Therapeutic and nursing measures

- Monitoring

A study by Bihari and colleagues [603] found that the main cause of sleep disruption on the ICU was the noise arising from discussions and chatting amongst medical staff.

Fostering the day-night cycle in weaning patients, especially those in the prolonged weaning process, seems reasonable from a physiological perspective, despite the negative results reported by Huttmann et al. [602]. For this reason, the creation of an atmosphere that promotes a normal day-night rhythm in critical care units has been identified as a structural criterion for weaning units (see criteria catalogue for the certification process in "WeanNet", the German network of weaning units; the catalogue is only available in German language) [604]. A particular level of importance is placed on the effort to either significantly reduce or completely eliminate asynchronies between the ventilator and the patient's breathing and care-related disturbances that disrupt the physiological circadian sleep-wake rhythm. There are a number of different measures available that keep the patient awake during the day in order to increase the pressure to sleep at night. Wherever possible, nocturnal disturbances arising from noise and light should be avoided in the ICU. This also includes nursing measures that can potentially disrupt the patient's sleep but are not absolutely necessary.

Concepts for improving sleep quality on intensive care units also include sleep-promoting measures such as light and noise reduction, and the provision of earplugs and eye masks [146, 605]. Specific pharmacological aspects regarding analgesia and sedation hold less importance in comparison to non-pharmacological measures, since several studies have shown that they prevent normal sleeping patterns [606, 607].

5.6.3.1. Significance of Melatonin. The American guidelines on the topic of analgesia, sedation and management of delirium do not provide any recommendations regarding the use of melatonin for improving sleep in intensive care patients [608].

Three randomised controlled trials with a collective patient population of 60 showed no clear effect of melatonin on sleep quality or duration $[609,610,611]$.

Ramelteon, a melatonin receptor agonist approved for use in the USA, was found to have favourable effects on the occurrence of delirium, but not on sleep quality [612, 613].

\subsection{Features of Spinal Cord Transection (SCT)}

The weaning of patients with upper-level spinal cord injuries is a challenge for specialised centres for many reasons. Since this usually pertains to the prolonged, discontinuous weaning of tracheotomised patients [614, 615], the literature reports the duration of stay on specialised units as ranging between 40 and 292 days [616]. Prolonged weaning processes are therefore the rule, since factors such as pulmonary infections delay the weaning process $[617,618]$. The failure rate after long-term weaning is mutually quoted at around $30 \%[614,615]$. Additionally diverse autonomic dysregulation conditions such as hypotonia, bradycardia, temperature dysregulation and autonomic dysreflexia can also consideraly hinder the course of treatment $[617,619]$ and requiring care from an experienced specialist. Respiratory weaning in upper-level spinal cord transection patients has been carried out in paraplegia clinics for the last 15 years.

\subsubsection{Pathophysiological Aspects}

Respiration is impaired in all patients with upper-level spinal cord injuries [620]. This is rarely due to pre-existing lung diseases, and instead stems mainly from respiratory muscle pump impairment following partial or complete denervation of the respiratory/auxiliary muscles. The diaphragm should be mentioned first here, since it performs almost $100 \%$ of the remaining active work of breathing in these patients $[621,622]$. It is important to train this muscle and its (residual) functions carefully, methodically and, above all, without causing fatigue. This training ensures that specific muscle fibre types that are initially quickly fatigued can now yield a sustained output 
$[622,623]$. While this phenomenon has been studied in detail after phrenic nerve stimulation in paraplegic patients [624], it can also be used for the expansion of ventilator-free periods for spontaneously breathing patients. Here it is important not to reach the so-called fatigue point of the diaphragm, otherwise the weaning process will be sustainably delayed or not possible at all $[625,626$, 627].

\subsubsection{Special Features of Patients with SCT}

The majority of patients are quadriplegic or quadriparetic with an SCT below $\mathrm{C} 0-8$, Abbreviated Injury Scale (AIS) Type A, B or C (Table 22) [628]. Paraplegic patients with accompanying injuries or illnesses that impair respiratory performance.

5.7.3. Additional Exclusion Criteria for Beginning the Weaning Process

In addition to the recognised general exclusion criteria, there are medical conditions typical for paraplegia under which weaning should not begin or be continued:

- Persistent, complete diaphragmatic paralysis without coughing capacity

- Untreated, high-grade pronounced autonomic dysreflexia

- Non-compensated spasticity that significantly impairs breathing

- Decubitus ulcers accompanied by sepsis

\subsubsection{Practical Course of the Weaning Procedure}

The weaning process is generally started with the patient in the supine position. The reasons for this are that spinal cord transection patients show a more stable blood pressure ratio as well as a higher breath volume in this position $[628,629]$. Starting at a spontaneous breathing time of $20 \mathrm{~min} /$ hour, weaning can generally be carried out in the sitting position (e.g., in a wheelchair), due to the trained diaphragm $[615,623]$. Spirometric monitoring of tidal volume and capnometry are nonetheless warranted at all times.

During the night, the patient's respiratory muscles are preferably completely unloaded until the next training cycle through the use of a controlled ventilation mode.

During the day, the weaning procedure is carried out as follows: A weaning cycle is planned for $12 \mathrm{~h}$ (e.g., 8 a.m. -8 p.m.). This weaning phase is discontinuous, since each hour offers both spontaneous breathing and muscle unloading during MV. This therefore results in up to 12 training units per day. The so-called averaged tidal volume (average tidal volume spirometrically measured
Table 22. The abbreviated injury scale (AIS)

\section{AIS Neurological deficit}

A Complete loss of motor and sensory function at S4/S5

B Sensory Incomplete. Sensory but not motor function is preserved below the neurological level and includes the sacral segments S4-5

C Motor incomplete function is preserved at the most caudal sacral segments for voluntary anal contraction (VAC) and less than half of key muscle functions below the lesion Level have a muscle grade $\geq 3$

D Motor incomplete status as defined equal to type C, with at least half (half or more) of key muscle functions below the lesion Level having a muscle grade $\geq 3$

E No motor or sensory deficits

across 20 spontaneous breaths) plays an important role in ascertaining the increments in the proportion of spontaneous breathing phases. Further determining factors include vital capacity, respiratory rate, extent of diaphragmatic mobility (based on sonography) and assessment of coughing capacity.

Important termination criteria for spontaneous breathing are the reduction in tidal volume (average TV minus 30\%) as well as the development of autonomic symptoms (spasticity, dysreflexia). Using these parameters and based on the clinical experience of the practitioner, the incremental units should be checked and corrected (if necessary) every day.

If daytime weaning is successfully completed (i.e., the patient's daytime respiration is stable for around 1 week), nocturnal weaning begins as follows: The spontaneous breathing phase becomes extended by $1 \mathrm{~h}$. This gives the patient the choice of whether to incorporate this extended time into the night, or be disconnected earlier from the ventilator in the early hours of the morning.

Standardized protocols have proven to be helpful for the weaning process and should be used as part of the documentation [615].

\subsection{Special Aspects in Paediatric Patients}

There are only a limited number studies addressing the topic of weaning, readiness-to-wean, and extubation in childhood. The following section is devoted to weaning in paediatric patients. Studies on prematurely-born and newborn infants are not included. 


\subsubsection{Weaning Protocols}

Two prospective randomised studies $[630,631]$ and retrospective analyses $[632,633]$ verify the use of a weaning protocol as a means for reducing weaning duration; however, this could not be confirmed by another randomised study [634]. A small retrospective study describes a weaning protocol for patients with SMA1 that includes the use of an in-/exsufflator (see also [553, 635]). They found that extubation is likely to be successful if the $\mathrm{SO}_{2}$ level is $>94 \%$, with an $\mathrm{FiO}_{2}$ of 0.21 ; pre-determined intervals of tracheal suctioning could be adhered to, and extubation was followed by NIV [636]. The approach described in this study increased the rate of successful extubation. In the meantime, the first results of a closed-loop automated weaning procedure have become available for children $[637,638]$, although the potential advantages of this system are yet to be demonstrated [639]. Even when the use of weaning protocols is likely to be beneficial in children, the extent of the available literature is not adequate enough to serve as a basis for their recommendation.

\subsubsection{Assessment of Weaning Ability}

The standardised assessment method for weaning ability in short-term ventilated children was investigated in a randomised study. Numerous parameters were evaluated on a daily basis $\left(\mathrm{FiO}_{2} \leq 0.5\right.$, PEEP $\leq 8 \mathrm{~cm} \mathrm{H}_{2} \mathrm{O}$, no new infiltrates observed in CT scans, no prolonged sedation or paralysis, normal electrolyte levels, hemodynamic stability and $\mathrm{Hb} \geq 8 \mathrm{~g} / \mathrm{dL}$ ) and an SBT was subsequently carried out if required. This approach reduced the duration of MV significantly [631]. A daily standardised assessment of weaning ability is recommended by expert groups [640]. It is generally agreed that besides the factors that are also applicable to adults, readiness-to-wean in children requires a sufficient hydration status [641, 642]. However, it remains to be determined whether a restrictive fluid management program is more favourable than a liberal one in terms of extubation outcome [643-645].

\subsubsection{Sedation and Sedation Protocols}

Sedation is usually required during invasive ventilation of children, due to their generally low level of tolerance for ventilation per se. One particular study reports an association between extubation failure and deep sedation [634], whilst others show that the use of sedation protocols for children allows the depth of sedation to be easier quantified $[646,647]$, as well as better controlled [648-651]. A randomised study showed that daily inter- ruption of the sedated state (inclusion criteria: $\mathrm{MV}>48 \mathrm{~h}$, peak inspiratory pressure [PIP] $<29 \mathrm{~mm} \mathrm{Hg}$ ) reduced the duration of MV [652]. However, in another study, the use of both a sedation protocol and a standardised assessment of weaning ability did not reduce the duration of weaning [648].

\subsubsection{SBTs and the Prediction of Successful}

\section{Extubation}

One study in children could not identify any difference in predicting weaning failure between an SBT with a Tpiece, and MV with PSV $2 \mathrm{~h}$ prior to extubation [653]. Additional conventional parameters for extubation failure such as $\mathrm{Vt}$, respiratory rate, $\mathrm{P}(\operatorname{imax})$, and $\mathrm{f} / \mathrm{Vt}$ in the SBT did not predict extubation failure sufficiently [654656]. The conduction of a 15-minute SBT in one study had a positive predictive value (PPV) of $92 \%$ for successful extubation [657]. The rate of extubation failure was, however, not lower than that found in a historical control cohort with clinically determined extubation readiness. Daily assessment of weaning readiness combined with an SBT reduced the duration of MV in short-term-ventilated patients, albeit without affecting reintubation rate [631]. Daily performance of the "RESTORE Extubation Readiness Test", which amongst other parameters includes a 2-hour spontaneous breathing phase, had a PPV of 92\% (89-95) for successful extubation [658]. Other predictors for successful extubation, such as an RSBI $<8$ (or $<11$ ) breaths $/ \mathrm{min} / \mathrm{mL} / \mathrm{kg}$ and a CROP (compliance, respiratory rate, arterial oxygenation and $\mathrm{P}_{\text {imax }}$ )-index $>$ 0.15 (or $>0.1$ ) $\mathrm{ml} / \mathrm{kg} /$ breaths $/ \mathrm{min}$, and $\mathrm{V}_{\mathrm{d}} / \mathrm{V}_{\mathrm{t}}$, have yielded controversial results [659-667]. A more recent prospective study observed that the tension-time index has a high predictive value for weaning success [668]. One problem associated with the evaluation of respiratory stability at the CPAP/T-piece junction arises from the use of thin paediatric tubes and the accompanying increase in resistance that results from the 4 th power of the radius. Extubation often leads to a decrease in airway resistance, so that it can still be successful despite a negative SBT. For this same reason, children often become exhausted during the SBT. On the other hand, compensating for the tube by increasing PSV levels for smaller endotracheal tubes during the SBT possibly overestimates readiness for extubation [669]. Therefore, no reduction in extubation failure could be achieved by performing standardised SBTs in children, suggesting that their regular use is not necessary.
Schönhofer et al. 


\subsubsection{Types of MV in Weaning}

One randomised study found no difference between weaning with PSV, VC, or without a protocol [634]. Another study that included 70 children found that the duration of MV did not differ, regardless of whether PCV or SIMV with PSV was used [670]. A pilot study showed that the use of NAVA accelerates the weaning process [671]. Based on these limited findings, a recommendation for a particular mode of weaning cannot be given at this point. However, by way of analogy with adult medicine, the use of the SIMV mode in patients undergoing prolonged weaning is not advisable.

\subsubsection{Post-Extubation Stridor}

Due to the narrow airways, stridor/ upper airway obstruction (UAO) is a common cause of extubation failure in children [129, 672-674]. Estimating the risk for UAO after extubation using the cuff-leak test $[198,675]$ or analysis of tube leakage with inflated tubes is disputed [675677]. Although limited by low case numbers, one particular meta-analysis reports that the prophylactic use of steroids reduces the frequency of post-extubation UAO and possibly reduces the rate of extubation failure $[678,679]$. Patients at increased risk (including those with long periods of MV [680], multiple airway procedures, laryngotracheitis/epiglottitis, positive cuff-leak test) may benefit from steroid treatment prior to extubation [681]. The use of a helium-oxygen mixture is an additional means of reducing the reintubation rate in children [682-684]; however, randomised controlled studies on this approach are currently lacking.

\subsubsection{Tracheotomy}

A tracheostomy is often indicated to enable weaning in infants or very young children with stenoses or malacia of the respiratory tract $[132,133,685]$. Due to the narrow airways, closure of the tracheostoma is only possible again when the child is of a sufficient size and has recovered from the underlying illness $[136,686,687]$. The rate of decannulation is low in patients with underlying neurological disease [688]. The use of early tracheotomy as a weaning strategy in severe respiratory failure such as childhood ARDS has received little attention. It is mostly only implemented for the transition of non-weanable patients to out-of-hospital MV and often only established after more than 60 days of MV [689-692]. Older children and adolescents could potentially benefit from the weaning strategy of an earlier tracheotomy, which has already been successfully applied in adult patients $[416,693]$. However, the results of a small retrospective study do not validate this notion [692]. Therefore, no general recommendation regarding this approach can be made. Due to the narrow airways and the use of small, short tubes, the risk of tubal dislocation is increased. Easy recannulation and a stable airway without a tube may be a life-saving measure [694]. Therefore, tracheotomies in children need to be surgically epithelialised. The area around the tube should be subjected to regular endoscopic examination in order to treat granulations [695], malacia, and dilatation of the posterior tracheal wall at an early stage, and to avoid a delay in repositioning. During long-term MV, cuffless cannulas are imperatively better for speaking ability, aeration of the nasopharynx and improved swallowing. In the event of high leakage and subsequent frequent activation of device alarms, cannulas with highpressure cuffs can be inflated at night and deflated during the day. For definitive weaning from the tracheal cannula, the use of successively smaller tubes over several weeks, or the use of a pre-fabricated or plastically adapted tracheostomy retainer in older children has proven to be successful if performed before the stoma is completely sealed with colloid bandage and surgically closed off. Closure of the tracheostoma is rarely successful in children under 2 years of age, due to the small size of the trachea.

\subsubsection{Non-invasive Ventilation (NIV)}

NIV is successfully used for weaning children from the ventilator [686, 696-698]. Although the use of NIV in long-term weaning has been described in a small paediatric cohort [686], there are no randomised studies on NIV in the weaning process in children outside the neonatal period. This means that a general recommendation for NIV cannot currently be made for children, even though NIV serves as an integral part of the weaning process in their adult counterparts. Immediate extubation to NIV seems more beneficial than rescue in the event of extubation failure, and is recommended in anticipation of difficult extubation $[697,699]$.

\subsubsection{High-Flow Nasal Cannula (HFNC)}

The HFNC is increasingly used during the post-extubation phase. According to randomised studies in adult patients, the HFNC was shown to be more advantageous than low-flow oxygen, but similarly as effective as NIV [456-458, 463]. The HFNC had no benefit in relation to the reintubation rate in paediatric cardiac surgery patients [700]. To date, the data situation remains too uncertain to estimate the importance of HFNC in childhood weaning. 
R39: Prophylactic steroid treatment prior to extubation is suggested for children who are at an increased risk of developing post-extubation stridor.

$R 40$ : Surgical epithelialisation is suggested for paediatric tracheotomies, and the position of the cannula should be regularly monitored using endoscopy.

R41: Daily interruption of sedation is suggested for children to reduce the duration of MV.

$R 42$ : Daily assessment of readiness to wean is suggested.

R43: The implementation of NIV directly after finishing invasive MV is suggested for paediatric patients in prolonged weaning.

\section{Infrastructure and Process Organisation for the Care of Patients in Prolonged Weaning: A Continuous Treatment Concept}

\subsection{Introduction}

The number of patients in prolonged weaning, which is defined by at least 96 hours of ventilation, is continuously growing [701]. This is also combined with an increase in the number of patients who require a prolonged weaning process, along with all the personal and financial implications for the health system [9] that accompany it. In Germany, in August 2015 there were approximately 27,500 intensive care beds [702] across 1177 hospitals that offer intensive care treatment; this amounts to just 34 beds/1000 residents. Even though ICU patients in prolonged weaning play a quantitatively smaller role (approx. $10-20 \%)$ [9, 32, 38, 703-706], high demands are placed not only on the specialised training of medical and nursing personnel, but also on the infrastructure and existing resources for treating these patients. Due to the different clinical situations that can lead to prolonged weaning and the subsequent heterogeneity of the areas from which patients in prolonged weaning are recruited, the infrastructural and organisational requirements for the treatment of patients in prolonged weaning vary amongst the corresponding wards or facilities, be it an acute-care hospital, a specialised weaning unit, or in a neurological-neurosurgical early rehabilitation setting with MV. On the basis of this structural plurality, the development of quality criteria is necessary to ensure that patients in prolonged weaning receive the required quality of treatment, regardless of the area in which the weaning process is carried out. During the process of caring for patients in prolonged weaning, it may turn out that patients need to be transferred to a specialised ward, unit or centre; this can be due to factors such as clinical indication, lack of capacity, or because the necessary resources for prolonged weaning are not adequately reflected in acute medicine. Nevertheless, an increasing number of patients with out-of-hospital invasive MV are being transferred directly from the ICU to out-of-hospital intensive care units (in Germany approx. 85\%), without the indication for out-of-hospital ventilation having been evaluated by appropriately qualified experts in prolonged weaning [707]. In order to establish or maintain a high quality of care, patient transfer absolutely needs to occur under a defined transition and discharge management process with defined quality indicators, whereby not only non-clinical structures, but also specialised clinics or weaning centres must be included [708]. This is illustrated by a recommendation from the $\mathrm{S} 2$ Guidelines for "Non-Invasive and Invasive Home Mechanical Ventilation for Treatment of Chronic Respiratory Failure", which proposes limiting the funding of out-of-hospital ventilation to a maximum of 3 months in patients in whom the need for invasive HMV is not confirmed by an expert centre [3].

\subsection{Acute-Care Hospital}

In intensive care medicine, the treatment of patients usually represents a continuum, starting in many cases with invasive MV and multiple organ failure. With the restitution of individual organ functions, weaning from invasive MV increasingly becomes the focus of the intensive care treatment, accompanied by (rehabilitative) measures to restore functionality. Therefore, as already mentioned in Chapter 5.1, weaning from invasive MV is included in therapeutic planning right from the start, and successful weaning from $\mathrm{MV}$ is an integral part of the comprehensive therapy concepts in acute intensive care medicine. Furthermore, acute intensive care medicine plays an important role in the weaning process in terms of preventing long-term MV and prolonged weaning. According to Kastrup et al. [708] this is where the track can be switched to successful weaning in the so-called process chain of weaning. A treatment plan based on defined quality indicators [708] increases the chances of prolonged weaning being successful, even within an acute intensive care medicine setting (see Chapter 5.1). However, due to the current increase in the number of patients with invasive out-of-hospital ventilation, it is necessary, in addition to using a strategy to avoid prolonged weaning, to create a sufficient number of appropriately specialised units in which patients who were primarily deemed "unweanable" are given another chance at weaning from
Schönhofer et al. 
the ventilator before they are transferred to an outpatient long-term care facility. Such concepts already exist in areas including respiratory medicine, neurological/neurosurgical early rehabilitation, or the acute intensive care medicine units that have become particularly specialised in this kind of chronic care.

\subsection{Weaning Units and Weaning Centres}

For patients in prolonged weaning (especially those in Categories $3 b$ and $3 c$; see Chapter 3.4) who show a high number of co-morbidities [709], or suffer from chronic critical illness [710] in addition to the typical weaning issues, treatment is required to take place in specialised units or centres with the appropriate interdisciplinary expertise of all the professional groups involved as well as the necessary structural prerequisites. Due to the heterogeneous clinical profiles of the patients and the different structural organisation of the hospitals and associated scientific societies, no single discipline can claim to be the sole representative of weaning units.

The accomplishments of specialised weaning centres are by definition not prospectively randomly investigated, but are rather based on analyses from single centres or databases from networks. For example, based on an evaluation of the WeanNet database, it was shown that of the patients who were externally transferred to a WeanNetcertified centre, $34 \%$ came from an internal medicine ICU, 22\% from an anaesthesiological ICU, and $11 \%$ from a surgical ICU [38].

It was also shown that approximately $60 \%$ of patients could be successfully weaned from invasive MV after a period of prolonged weaning [38]. In addition, a significant proportion of pneumological patients in prolonged weaning who suffer from chronic respiratory failure can be continue to be cared for in an outpatient setting after switching from invasive to non-invasive MV in a weaning centre [12].

These findings were used as a basis by the Sk2 guideline "Non-Invasive and Invasive Home Mechanical Ventilation for Treatment of Chronic Respiratory Failure" [3], which define a weaning centre as centre for MV which specialises in patients with prolonged MV and consists of the following units:

- Intensive care unit

- Weaning unit

- Home MV unit

However, these specifications are not equally suitable for all areas in which prolonged weaning takes place. Using intensive care areas of the German Society of Anaesthesiology and Intensive Care Medicine (DGAI) as an ex- ample, the structure of care provision within acute intensive care medicine is instead represented by a modular weaning concept. Depending on the different levels of care, the specialised intensive care and ventilation therapy expertise required for the prolonged weaning process is defined and maintained either as an integrated part of an intensive care unit, or on a separate weaning ward $[711,712]$.

Due to the superordinate structural organisation within the different hospitals, specific services can be provided through interdisciplinary and (if necessary) inter-hospital collaborations between specialists.

\subsubsection{Special Features of Paediatric Patients}

In large children's hospitals, the weaning process or transition to long-term MV usually takes place on the paediatric ICU. Specialist training in this area is desirable. Intermediate care with child-friendly surroundings would be desirable, especially for the transition to out-ofhospital ventilation.

The hallmarks of a specialisation in paediatric weaning and transition should be:

- Appropriate facilities for and expertise in paediatric intensive care medicine, in-/out-of-hospital MV, treatment of neuromuscular diseases, transition to ambulatory treatment

- Experience with NIV in children

- Experience in the management of tracheal cannulas, including an ability for performing bronchoscopy

- Consultational support from ENT medicine

- Paediatric thoracic surgery, radiology and gastroenterology

- Optional: access to polysomnography lab, as well as an outpatient clinic for ongoing care after discharge The structural prerequisites are:

- Child-friendly surroundings

- A continual presence in the clinic or availability for direct contact by nursing staff (due to the children's potential anxieties)

- Teacher/activity room

- School for ill children

- Physiotherapy

- Residency for parents as well as an option for "rooming in"

- Psychological support for parents

A specialisation in paediatric prolonged weaning should be reflected in the corresponding number of cases (at least 10 cases of paediatric prolonged weaning per year). The following recommendations are based on the views of the expert group: 
R44: Existing expertise in diverse paediatric sub-disciplines is recommended for a specialisation in weaning and the transition to out-of-hospital MV.

R45: A child-friendly environment and staff that specialises in the care of paediatric patients are suggested as structural prerequisites.

\subsection{Early Rehabilitation}

According to the position paper on interdisciplinary early rehabilitation, this occurs at the earliest time possible during the acute-care phase in hospital: "Early rehabilitation should be understood as the first link in a seamless rehabilitation process chain, and at the same time as an integral, legally-required component of hospital treatment" [713]. Therefore, the early rehabilitation process in prolonged weaning already begins under optimal conditions during the acute intensive care treatment phase, and is then continued in specialized early rehabilitation facilities after the acute treatment phase.

Under the same umbrella term of early rehabilitation, 2 different systems have developed in the areas of neurology and pulmonary medicine. This development takes into account the fact that the underlying neurological and pneumological clinical pictures require different therapeutic priorities and work with different time windows. For example, early neurological rehabilitation facilities are places where patients are often still in the process of weaning from MV, whereas early pulmonary rehabilitation facilities mainly focus on further rehabilitation of patients who have already been weaned.

\subsubsection{Special Characteristics of Early}

\section{Neurological-Neurosurgical Rehabilitation}

For several decades, the care of critically-ill patients in the neurological-neurosurgical early rehabilitation phase has been guaranteed by combining neurological, neurosurgical, intensive care and neurorehabilitative competence. The early-rehabilitation patients treated here are not only at risk of complications at the intensive care level, but even more so at risk of neurological or neurosurgical complications (e.g., increased intracranial pressure or epileptic seizures), which then require the permanent involvement of neurological and neurosurgical expertise in the early rehabilitation process [233]. By applying a therapy concept that is attuned to specific problems, a multi-professional team can guarantee a treatment plan that is geared towards activity and participation disabilities. The restoration of respiratory function as well as successful prolonged weaning are important therapy goals.
Table 23. Phase model of the federal working group for rehabilitation

\begin{tabular}{ll}
\hline Phase & Description \\
\hline A & Acute treatment phase \\
\hline B & $\begin{array}{l}\text { Treatment/rehabilitation phase in which intensive care } \\
\text { treatment options must be maintained }\end{array}$ \\
\hline C & $\begin{array}{l}\text { Treatment/rehabilitation phase in which the patients can } \\
\text { already participate in the therapy, but still require curative } \\
\text { medical measures and intensive nursing care }\end{array}$ \\
\hline D & $\begin{array}{l}\text { Rehabilitation phase following conclusion of early } \\
\text { mobilisation (medical rehabilitation in the traditional }\end{array}$ \\
sense)
\end{tabular}

Since the outcome of neurological patients (e.g., following a stroke) can be improved by early transfer to an neurorehabilitative facility [714], the following recommendation was made with strong consensus in the 2017 S2k Guidelines for prolonged weaning in early neurological/neurosurgical rehabilitation: "Ventilated patients with diseases of the central and/or peripheral nervous systems and/or neuromuscular diseases should be transferred as early as possible to neurological-neurosurgical early rehabilitation facility with expertise in intensive care medicine and weaning" [233].

Within the phase model of the Federal Working Group for Rehabilitation, neurological-neurosurgical early rehabilitation is assigned to Phase B (see Table 23), which means that it takes place directly after the acute treatment phase (Phase A) [715]. However, according to the reimbursement system of the statutory health insurance companies, this is a hospital-based treatment (according to social code $\$ 39$ SGB V) with its own procedure (Operation and Procedure Code - OPS 8-552) [716] within the DRG (Diagnosis-Related Groups) system.

In the meantime, there are several studies that provide real-life data on weaning in neurological-neurosurgical early rehabilitation. Based on these data it can be concluded that weaning from MV is successful in 70-92\% of cases [564, 717-719], after an average of 2-3 weeks [717, 718]. The mortality rate ranged between 6 and $23 \%$, where two-thirds of the patients died during palliative therapy 
Table 24. Distribution of the patients $(n=190)$ in early pulmonary rehabilitation according to ventilation status and (where relevant) ventilation interface [720]

\begin{tabular}{llc}
\hline Ventilation status & On admission, \% & At discharge, \% \\
\hline Invasively ventilated & 11 & 5 \\
NIV & 38 & 44 \\
No MV & 51 & 51 \\
\hline
\end{tabular}

[564]. The data available to date suggest that the burden of disease has a considerable influence on weaning from MV [719]. Although there is no dose-response effect regarding the effectiveness of early rehabilitation intervention, this is recommended in studies that primarily investigated non-neurological intensive care units [233].

\subsubsection{Special Features of Early Pulmonary}

Rehabilitation

Pulmonary rehabilitation is also a hospital treatment according to $₫ 39$ SGB V, and corresponds to the OPSCode 8-559 in the German DRG system. In general, patients are treated here after acute MV as well as with or following chronic MV. Usually there is an underlying chronic pulmonary or respiratory muscular disease, or sequelae following complicated MV or prolonged weaning.

6.4.2.1. Patient Population. The German Respiratory Society (DGP) primarily recommends performing the prolonged weaning procedure in specialised weaning centres, and then following this phase with early pulmonary rehabilitation. While almost all neurological patients (96\%) who underwent early neurological rehabilitation are on invasive MV [718], this ratio is markedly lower for patients in early pulmonary rehabilitation, as shown in Table 24.

According to the study by Dellweg et al. [720], a total of $94 \%$ of patients had previously received invasive MV, and in $29 \%$ of all cases, the ventilation status changed during the early rehabilitation phase. The diagnoses that led to MV were distributed as follows: Infections and sepsis (31.1\%), COPD exacerbations (24.2\%), elective surgery $(22.1 \%)$, emergency operations $(8.9 \%)$, heart diseases (6.3\%), neurological diseases $(4.2 \%)$ and other pulmonary diseases (not COPD, 3.2\%). The overall mortality rate of $4.2 \%$ during rehabilitation indicates the severity of the disease.

In general, patients undergoing prolonged weaning both in the long phase of their acute illness and in the

Prolonged Weaning: S2k Guideline by the German Respiratory Society prolonged weaning phase, experience a variety of functional limitations that can persist for some time after their stay in the ICU. These mainly include functional sensory and neurocognitive deficits. Muscle weakness that is acquired during the ICU stay (ICUAW) worsens the longterm mortality rate, increases the 1-year risk of mortality and is accompanied by an increased use of resources [67, 116] (see Chapter 4.5). Patients who already experienced delirium on the ICU (ICU-delirium) have significantly lower activity-of-daily-living (ADL) scores, as well as poorer motor and sensory function after one year, in comparison to patients who did not have ICU-delirium [721]. From a pneumological perspective, it is important that motor weakness is not just limited to the trunk muscles, but also affects the respiratory muscles to at least the same extent [120]. In this respect, Dellweg et al. showed that the outcome after early pneumological rehabilitation in terms of mobility (walking distance) and ADL scores (FAM (Functional Assessment Measure)/Barthel-Index) is comparably good for patients without MV versus those with non-invasive and invasive MV [720]. Another important component from a prognostic and therapeutic point of view is the perception and treatment of dysphagia (see Chapter 5.6.1.5) [722].

6.4.2.2. Neuromuscular Spontaneous Breathing Capacity in Early Rehabilitation. Persistent respiratory muscle weakness occurs more often than peripheral muscle weakness after prolonged weaning. Dres et al. [120] detected diaphragmatic dysfunction in $92 \%$ of difficult-to-wean patients, whereas ICU-AW was only present in $46 \%$ of these patients. For patients in prolonged weaning, the corresponding rates actually reached 100 and $50 \%$, respectively [120]. This underscores the importance of monitoring, diagnosing and treating respiratory muscle function independently. As in weaning, the goal of pulmonary early rehabilitation continues to be the improvement respiratory muscle competency and the minimisation of respiratory muscle loading. In this context, the type of ventilatory support may well change during the rehabilitation phase, as evidenced in Table 24. Whether NIV remains necessary during the patient's convalescence can only be determined by a withdrawal attempt. This attempt should be evaluated at least 7, but preferably 14, days after a MVfree period, since renewed decompensation following successful NIV can take a relatively long time [723]. In a cohort of hypercapnic COPD patients who were treated with NIV during early rehabilitation, it could be shown that besides an increase in walking distance, there was also a reduction in $\mathrm{CO}_{2}$ values, suggesting an improvement in respiratory pump endurance [724]. However, this study 


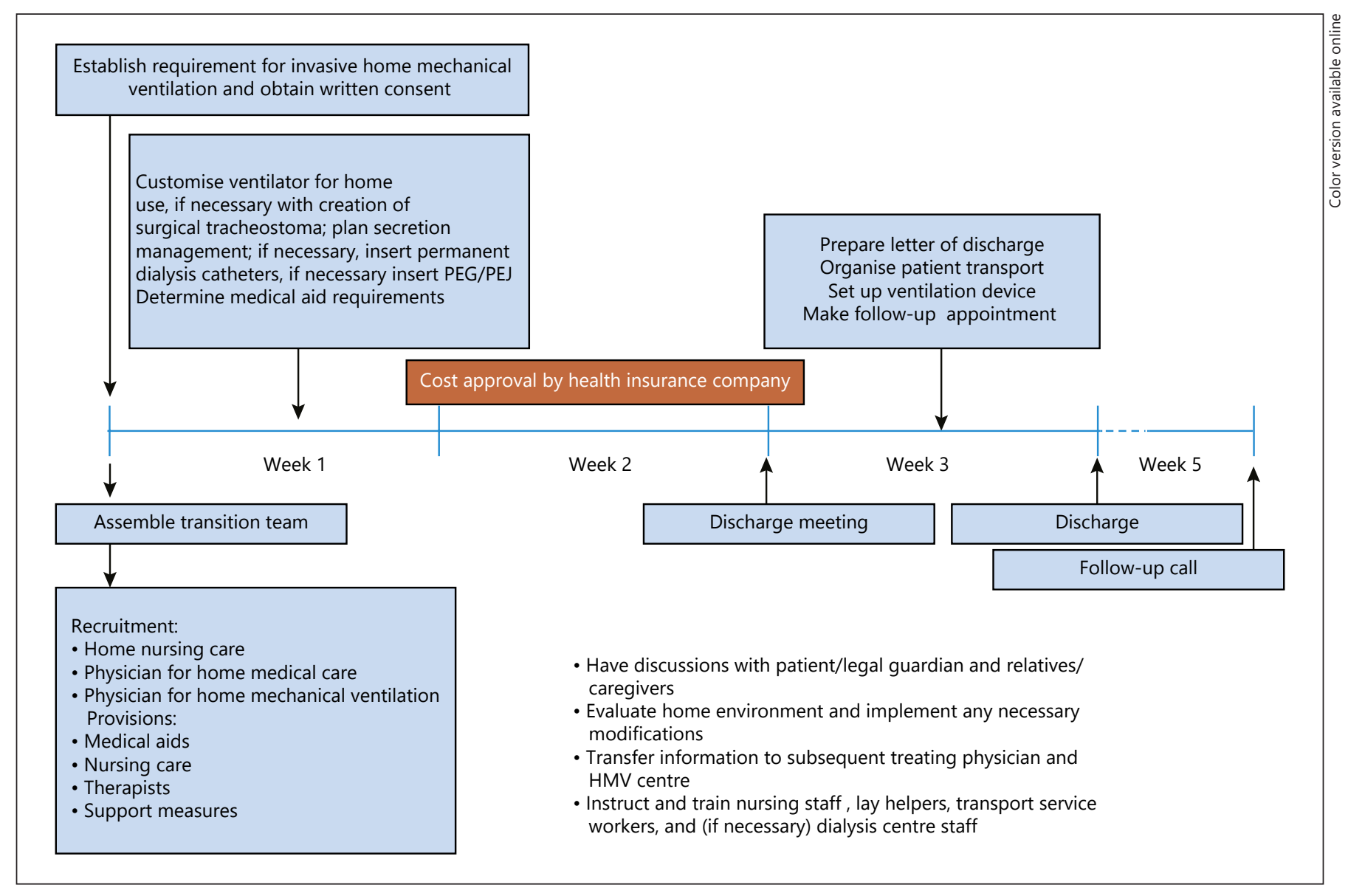

Fig. 8. Chronological sequence of events in transition and discharge management [rerif].

also showed that additional inspiratory muscle training (IMT) can once again significantly lengthen walking distance and increase respiratory muscle strength and power [724] (also see Chapter 5.6.1.6.1). IMT can also be carried out with a tracheal cannula in place. Bissett et al. [575] showed in a cohort of weaned patients who were still tracheotomised that respiratory muscle strength and quality of life had significantly increased in the group of patients who received IMT therapy.

6.4.2.3. Transition from Pulmonary Early Rehabilitation. The spectrum of findings related to early pneumological rehabilitation is very broad, ranging from continued dependence on invasive MV with a high level of nursing care, to occupational reintegration.

Therefore, the Phase B rehabilitation period should consist of continuous, individualised check-ups in order to identify any further rehabilitative steps that are indicated for the patient. The associated applications for funding should be submitted to the relevant agencies in a timely manner. The S2k "Guidelines for Non-Invasive and Invasive Home Mechanical Ventilation for Treatment of Chronic Respiratory Failure" contain further details regarding the logistics of managing the transition of invasively-ventilated patients [3]. In patients with pathogens that require isolation, the organization of further disposition or rehabilitation measures is especially problematic and protracted. The same applies for patients on non-invasive and (especially) invasive ventilation. This is also where the cost bearers should be included at an early stage of planning [720].

\subsection{Management of Transition and Discharge}

The transition process is schematically presented in Figure 8.

In the process chain of prolonged weaning, it is essential that the discharge phase is managed adequately and 
that monitoring of the process includes an evaluation for another attempt at weaning in cases where the potentially reversible causes of weaning failure show improvement $[3,708]$; this allows a reduction in the number of out-ofhospital invasive MV cases that are not/no longer indicated.

This particular approach is imperative, especially because expertise in MV or in handling trachael cannulas cannot invariably be provided by physicians in private practice [3]. Competency in NIV is essential for a weaning centre/unit; however, when this expertise cannot be provided by the weaning centre itself, an inter-hospital collaboration with another centre with appropriate expertise is an alternative. In this instance, it is particularly important that indications for the initiation of NIV and the management of discharge all work in temporal cohesion with the weaning process. Experience has shown that problems often occur at these interfaces when geographical distance exists. More details on the indication process, as well as the initiation, technical aspects and transition into out-of-hospital ventilation can be found in the S2k Guideline [3].

\subsection{HMV and Follow-Up Care after Prolonged Weaning}

After discharge from hospital, a considerable proportion of patients in prolonged weaning require intensive and chronic medical care, possibly with long-term nursing care, due to persistent ventilatory insufficiency that requires extra-hospital ventilation, or because of an ongoing need for a tracheal cannula. This means that the management of discharge in these patients is highly demanding, not only because the correct medical aids must be prescribed, but also because there needs to be a connection to the appropriate medical, therapeutic and nursing aftercare measures at the time of discharge. The person responsible for this is the discharging hospital physician [3].

The liberation of remote treatment was agreed upon on the so-called "Doctors Day" (in German language: "Ärztetag") in 2018 [725]. With the aid of telemedicine resources such as teleconference-based visits and appropriate contractual solutions for intersectoral collaboration, experts in outpatient MV working in centres for MV and/or weaning will be able to support physicians in private practice, as well as nursing personnel and patients in the outpatient sector. Even patients who have undergone prolonged weaning can benefit from this approach. Regular televisits from a centre for MV and/or weaning can identify patients with weaning potential, and guide them towards a renewed weaning attempt in a timely manner. In addition, televisits between intensive care units, rehabilitation clinics and specialized weaning centres could support the process of establishing indications for invasive out-of-hospital MV, and allow interdisciplinary conferences to become a mandatory element of discharge management.

\subsubsection{Category 3a I: Successful Weaning with}

\section{Decannulation}

Many patients in this category suffer from a number of severe diseases that require intensive medical care, even after successful weaning; therefore, ongoing ambulatory medical care needs to be ensured. In patients with a loss of autonomy and a subsequent nursing care dependency, ambulatory or nursing care should be arranged if rehabilitation therapy is not indicated.

\subsubsection{Category 3a II: Successful Weaning without}

Decannulation

Patients in this category often have a severe neuropsychological deficit such as that accompanying brain injury, dementia or Parkinson's disease. The ongoing need for a tracheal cannula usually results from dysphagia with an aspiration tendency and accompanying cough insufficiency. Those affected are characterised by a pronounced loss of autonomy with a high need for care, making independent care almost impossible. In addition to suctioning, tracheal cannual exchange and tracheostomal care, there is often the need for gastrostomal care, enteral nutrition and excretion control. The extent of autonomy loss determines the intensity of nursing care, the level of nursing qualifications, and the configuration of medical aids and instruments for monitoring. Continued physiotherapy, occupational therapy, and speech therapy can enable decannulation in the long term in some of these patients after all, thus underscoring the importance of access to medical specialists and suitable therapists for further therapy planning.

Another group of patients in this category is affected by an obstruction of the upper airways, glottis or trachea. If their autonomy is maintained, these patients may be able to learn the tracheal cannula care process themselves, or can be assisted by their caregivers (similar to cannula care after laryngectomy). If the loss of autonomy is due to functional motor impairment or cognitive deficits, these patients not only require specialist medical care for the causal disorder, but also the provision of suitable aids and nursing care, as well as a stable tracheostoma. 
6.6.3. Category 3b I: Successful Weaning with Long-

Term NIV, without the Need for Supplementary

Nursing Care

Patients with persistent respiratory failure after prolonged invasive ventilation can be stabilised in the longterm by means of mostly intermittent NIV, such that the initiation of NIV is indicated before discharge from hospital. The choice of medical aids is the responsibility of the prescribing doctor. The selection and adaptation of ventilation accessories purely through medical suppliers is strongly unadvisable. Affected patients who have retained their autonomy can receive intensive instruction in the clinic on the use of the required aids and devices, so that they can carry out the MV independently at home. For medical aftercare of patients with NIV, a long-term association with an appropriate centre for $\mathrm{MV} /$ weaning should be established. Further details on this topic are available in the S2k Guideline, "Non-Invasive and Invasive Home Mechanical Ventilation for Treatment of Chronic Respiratory Failure" [3].

\subsubsection{Category 3b II: Successful Weaning with}

Long-Term NIV, with the Need for Supplementary

Nursing Care

Patients with motor dysfunction or cognitive deficits potentially require assistance with NIV. In cases of slight loss of autonomy and intermittent, non-life-sustaining NIV, such help can be provided by caregivers or nursing staff who do not require additional special qualifications, but have been appropriately trained (e.g., putting on and taking off the mask, operating the ventilator, cleaning the accessories). In cases of high-grade loss of autonomy with the constant need for a carer during NIV, the affected patients are reliant on the support and permanent commitment of specially-trained personnel (e.g., removal of mask during coughing, dyspnoea or vomiting). Life-sustaining NIV can only be performed in the outpatient setting if a specially-trained person or skilled nurse is constantly present. The prescribing physician is responsible for evaluating the intensity of care required, as well as the need for specially trained personnel and, if necessary, the training of out-of-hospital care providers. This means that the indication and initiation of non-invasive outpatient MV with high care requirements should be carried out by a weaning or HMV centre with appropriate expertise. Under no circumstances should non-invasive outpatient MV be considered as a continuation of intensive care medical treatment. The regular or frequent presence of a physician, apparatus-based examinations or invasive therapy procedures cannot be guaranteed in the outpa- tient setting. For the care of patients with pronounced respiratory failure who require intensive nursing care, the prerequisites for discharge and the requirements for the doctors, nursing/medical aid staff and therapists providing ongoing support, are similar to those used in invasive HMV. This topic is discussed in detail in the S2k guidelines for non-invasive and invasive HMV for the treatment of chronic respiratory failure [3].

\subsubsection{Category 3c I: Unsuccessful Weaning with} the Continuation of Invasive MV in the Outpatient Setting

The prerequisites, course and aftercare associated with invasive home MV are presented in detail in the Sk2 Guideline, "Non-Invasive and Invasive Home Mechanical Ventilation for Treatment of Chronic Respiratory Failure." [3].

Due to the far-reaching consequences for the patient and its relatives and supporting community, the diagnosis of weaning failure should be made by a physician experienced in MV and weaning, before invasive HMV is initiated for the first time. When establishing an indication, ethical aspects and the patient's willingness to receive comprehensive information must be considered in addition to medical facts [708] (also see Chapter 7). The extent of nursing care is very high, and in many regions home-based care is rarely achievable due to the lack of nursing capacity. Due to the reduced number of staff, care in a specialized nursing facility is associated with an additional loss of autonomy, which should be taken into account when informing the patient. Due to the difficulties involved in transporting invasively-ventilated patients, medical home visits are necessary to ensure adequate medical care, and must be arranged before discharge from hospital. Due to this complex care situation as well as the different medical and nursing requirements for different clinical profiles or multimorbidity, invasive out-ofhospital ventilation should therefore, if possible, be initiated in a weaning or home MV centre. Such a centre can determine the feasibility of a particular treatment, take over the regular medical follow-up of these patients, and is available on an advisory basis for doctors in private practices. Quite a few of the affected patients can actually still develop weaning potential in due course, especially in cases of underlying muscle weakness after long-term intensive therapy, VIDD, stabilisation of co-morbidities, or regression of dysphagia. Regular assessment by experts is indispensable for recognising this potential, as well as for the targeted prescription of supportive physiotherapy or speech therapy. However, given the lack of scientific 
evidence, and for reasons of patient safety, weaning from the ventilator in the outpatient setting is obsolete [13, 726]. This is because weaning often requires invasive diagnostic and therapeutic measures that are usually not available in the out-of-hospital setting.

\subsubsection{Special Features of Paediatric Patients}

In the event of definitive weaning failure, the further prospect of long-term MV should be determined on an interdisciplinary basis, both in the best interest of the child and in close collaboration with the parents [727]. It is often the case that non-invasive home MV is sufficient for young children and infants [728]. When starting longterm MV, the treatment time on the ICU should be limited [729] and the family should be incorporated into the care process early on. Further details for this transition process can be found in [3]. In addition to this medical care, the family's ability to cope with the stress and the space available at home needs to be taken into account. During the ventilation periods, at-home care must be provided by a specialised nursing service or, if necessary, through trained lay helpers. Children undergoing lifesustaining MV should be supplied with at least 2 ventilator devices that can be adapted to paediatric physiology, as well as at least 2 suction devices. Pulse oximetry is indicated for monitoring during ventilation, while ECG monitoring is only carried out if there is a cardiological indication. An end-expiratory $\mathrm{CO}_{2}$ measurement may be necessary in rapidly changing ventilatory conditions, such as those occurring during frequent seizures or temperature instability [730, 731]. Further details on the medical, nursing and technical aspects of home-based care of long-term ventilated children can be found in [3].

In addition to medical treatment, the home-based nursing care of ventilated children should also focus on their overall development [732-734]. Appropriate measures for this aspect should already be a part of the discharge management process during the transition phase. Participation in special or regular kindergartens and schools is desirable even in ventilated children, although this can only be achieved with individual support. This linking of nursing and natural healing measures results in the need for a high number of personnel. Any additionally required support measures such as physiotherapy, occupational therapy, or speech therapy must be determined by the appropriate support agencies or social paediatric centres [732, 735].

Specialised care facilities can take over the role of caring for the child when care cannot be provided by the family itself. In addition to providing medical care, these facilities should ensure that children are supported on various levels. The prerequisites for this are child-friendly surroundings, reliable contact persons and appropriate support services. This can usually only be provided by facilities with appropriately trained staff that specialise in both childcare and long-term MV [3]. After being discharged to a home MV setting, children should be presented for regular check-ups at an MV centre with experience in paediatric ventilation; this allows not only for the adjustment of the ventilation settings in accordance with the altered physiological changes that occur due to growth, but also for weaning potential to be monitored, if necessary [733]. Predominantly ambulatory weaning that takes place in close conjunction with a specialised weaning centres has been described in the ambulatory setting in several publications [736, 737]. Long-term weaning is often a process that takes place over years [687].

\subsection{Structural and Procedural Quality Requirements in Prolonged Weaning}

There are different catalogues of requirements and certifications (DGAI certificate and WeanNet certificate of the DGP) for the structural and procedural quality requirements of units specialising in weaning $[604,711$, $712,738]$. In addition, the special features of patients in the neurological-neurosurgical early rehabilitation phase need to be taken into account, which makes the integration of neurological and neurosurgical expertise indispensable [233] (regulated by OPS 8-552, DIMDI OPSVersion 2018).

Due to different existing concepts, this guideline deliberately refrains from providing more detailed information on the structural organisation of the weaning unit; however, some of the requirements mentioned below should be fulfilled by a weaning unit or centre for patients in prolonged weaning.

\subsubsection{Requirements for Weaning Units or Weaning} Centres

The following general requirements should be met by specialised wards that treat patients in prolonged weaning:

- A sufficient case number of patients treated in weaning

- A team of personnel comprising an adequate number of medical and nursing staff that meets the requirement of an ICU. Deviations from this may be possible in patients with ventilation-dependent paraplegia.

- Competence in intensive care medicine, especially regarding ventilation during the weaning process as well 
as the complications that are frequently observed during the course of weaning

- Competence in the diagnosis and treatment of underlying and accompanying illnesses, due to the frequent, multiple co-morbidities that can occur

- Competence both in MV medicine and adjunctive measures, in order to provide the patient with the best chance of being weaned from invasive MV at the least

- Early engagement of the various professionals involved in weaning, such as physiotherapists, and speech, respiratory, and occupational therapists

- Competence in the diagnosis of chronic respiratory failure, potentially with the initiation of NIV after formal successful completion of weaning

- Establishment of structures for a cross-sectoral, interdisciplinary care of patients in prolonged weaning

\subsubsection{Number of Treated-Patient Cases}

A prerequisite for maintaining sufficient competence in the treatment of this often very complex group of patients is sufficient practice and experience, which is why a minimum number of 20 patients categorised to Group 3 need to be treated each year (DGAI requirement) (See Table 1 for group classification). According to the criteria catalogue for certification in WeanNet, 40 patients per year need to be treated in order to qualify (DGP requirement) [604].

\subsubsection{Staffing Requirements}

Recommendations regarding the number of nursing staff for intensive care and weaning wards have been published by various professional societies (please refer to the corresponding professional associations for official recommendations). This varies according to the specific circumstances of the area in which prolonged weaning is carried out. The transfer of tasks to the associated professional groups (physiotherapy, respiratory therapy and speech therapy) should be taken into account.

According to the DIVI and the DGAI, a specialist with expertise in intensive care medicine or a specialist with an additional qualification in intensive care medicine, respectively, should be present during core working hours.

In contrast to the acute medicine setting, a medical 3 -shift model is not absolutely necessary within the realm of the weaning unit. However, a medical shift model does need to be implemented in cases involving a high intensive care effort (e.g., with higher patient number).

The specifications for the nursing-patient ratio are regulated through the DGP- and DGAI-based criteria for certification of a weaning unit $[604,712,738]$.
A useful approach in prolonged weaning is an interdisciplinary one that integrates various branches including physiotherapy, occupational therapy and speech therapy, as well as respiratory therapy and psychological counselling. This is why specialists from these various professions are also recommended.

\subsubsection{Medical Expertise}

Due to the polymorbidity and disease severity of ventilated patients, the medical expertise of a weaning unit or centre should entail a high degree of knowledge in intensive care medicine, vast experience in treating the underlying and accompanying diseases, and an awareness of their course and prognosis (see Chapter 7). This expertise is usually only guaranteed within an interdisciplinary team. The minimum standard for weaning centres under different management should be the qualification of the head supervisor as a specialist physician with an extra qualification in intensive care medicine. ${ }^{1}$

With regard to the special requirements in the fields of spinal cord transection and neurological-neurosurgical early rehabilitation, please refer to the relevant literature $[233,739]$.

Furthermore, the presence of medical and nursing personnel with experience in intensive care medicine needs to be guaranteed on a 24 -h basis in the treatment unit. Regarding the qualification of nursing staff in intensive care units, a sufficient number of staff with specialist training should be aimed for $[38,604,711,712]$.

According to the WeanNet survey, the mortality rate in the weaning process is $14.9 \%$, which corresponds to a total of 1,027 out of 6,899 patients [38]. Many multimorbid patients, who are usually elderly with frequent complications (including those caused by infections or comorbidities), thus confront the weaning centre team with palliative medical issues. Appropriate expertise in dealing with terminally-ill patients must therefore be made available (see Chapter 7.1.2).

\footnotetext{
The topic of professional qualification in intensive care medicine for the head of a weaning unit was discussed in detail by all participants at the 2 nd consensus conference on 31.01.2018. The majority of delegates from the professional associations involved in the revision of the guideline argued that the head of a weaning unit or centre should be a qualified medical specialist with an additional qualification in intensive care medicine. The German Society for Neurorehabilitation (DGNR) countered this argument with reference to the guideline "Prolonged weaning in neurological-neurosurgical rehabilitation." [233]. At the end of the discussion, 17 out of 18 voters voted (with 1 dissenting vote) in favour of the statement that the head of a weaning unit or centre should be a qualified medical specialist with an additional qualification in intensive care medicine.
}

Schönhofer et al. 


\subsubsection{Technical Set-Up}

The technical set-up corresponds structurally to that of an intensive care unit, albeit with specific requirements; these include the promotion of a day-night rhythm, and sufficient space and equipment for early and intensive physiotherapy. Another particularly important point is NIV, whose importance in weaning is also discussed in the S3 guideline "Non-invasive Mechanical Ventilation in Acute Respiratory Failure" [16]. NIV plays a decisive role in prolonged weaning with frequent, persistent weakness of the respiratory pump, not only to reduce the number of patients who are on invasive MV when they are discharged to an outpatient facility, but also for humanitarian and financial reasons. According to data from WeanNet [38], approximately $19.4 \%$ of patients who are weaned in a pulmonary medical centre are discharged with NIV. Therefore, ventilators must be made available for NIV at home, in order to allow patients to adapt to them. An adequate supply of masks (nasal, nose/mouth and face masks), equipment and possibly active or passive humidification is essential for performing NIV correctly. More details on the technical requirements and set-up for both non-invasive and invasive HMV can be found in the S2k Guideline, "Non-Invasive and Invasive Home Mechanical Ventilation for Treatment of Chronic Respiratory Failure." [3]. Furthermore, the catalogues of requirements can be found in the DGAIand DGP-formulated lists of specifications for certification $[38,604,711]$.

Due to the epidemiology of the population and the high proportion of significantly overweight patients in the prolonged weaning group, special beds and chairs need to be made available for obese patients (weightbearing capacity of up to $220 \mathrm{~kg}$, even higher if the focus is on severely-obese patients). In some cases, special beds with a higher weight-bearing capacity are also required (usually on a daytime-lease basis). Walking frames that are specially approved for patients in this weight category are also desirable. A technical set-up with aids for early mobilisation is not only necessary for neurorehabilitation units and spinal cord transection centres, but also for other weaning units, since mobilisation is supported or even enabled in the first place through the use of aids such as bed pedal exercisers and vibration plates.

Regarding secretion management, different devices such as oscillating and non-oscillating PEP systems, mechanical in-/exsufflators and bronchoscopy equipment should be provided according to the recommendations outlined in the corresponding chapter of this guideline (see Chapter 5.6.1.4).

Prolonged Weaning: S2k Guideline by the German Respiratory Society

\subsubsection{Room Facilities}

There are 2 important aspects regarding the set-up of patient rooms, especially for planning a new weaning unit:

- Room size

- Maintenance of a day-night rhythm

- Single rooms with sufficiently large dimensions are advantageous for several reasons:

- Although exact figures are not available, a large proportion of patients in prolonged weaning is colonised with multi-resistant pathogens; therefore, these patients often need to be cared for in isolation (see Chapter 5.1.6)

- Space is also required for the sometimes complex care of patients, as well as for their mobilisation (e.g. bed pedal exerciser, wheeled walking frame, mobilisation whilst on invasive MV etc.)

- In addition, patients can occasionally suffer from delirium or are considerably altered psychologically during the course of the disease, such that occupying a room with several other patients is unfavourable

Since the day-night rhythm is an important adjunctive measure in the treatment of patients, the installation of special lighting can provide significant support for the therapeutic measures mentioned in Chapter 5.6.3 [740, 741].

Cross-sectoral outcome data for prolonged weaning are generally lacking. Previous studies have shown that it is relatively easy to compile outcome data, especially that on the success rate of the weaning process in relation the underlying disease and the pre-ventilation time. It is strongly recommended that each weaning centre/unit carries out such outcome analyses (in a network, if possible). Models are available for this purpose [742]. Data from WeanNet show, for example, that about $60 \%$ of the prolonged-weaning patients who are transferred to a respiratory weaning centre can be successfully weaned while avoiding further invasive MV; around $20 \%$ of these patients with chronic respiratory failure were discharged with NIV at home [38].

R46: After the completion of weaning in patients with relevant underlying or accompanying illnesses, diagnostics for the presence of chronic respiratory failure are recommended during the stay on the ICU. Follow-up measurements are recommended for the appropriate indications.

R47: In cases of chronic respiratory failure, it is recommended that the patients are offered NIV after primarily successful prolonged weaning, and that this is initiated with consent. 
QI4: After the completion of weaning in patients with relevant underlying or accompanying diseases who are at risk of ventilatory insufficiency, diagnostics for the presence of chronic respiratory failure are carried out upon discharge from the ICU or weaning unit/centre, and a renewed evaluation is recommended to the doctor providing ongoing care (Number of patients in whom diagnostics for the presence of chronic respiratory failure were performed/ Number of patients in whom diagnostics for the presence of chronic respiratory failure would have been indicated $\times 100$ ).

\section{Therapy Decisions for the End-of-Life Phase}

\subsection{Issues}

Patients are increasingly surviving intensive care treatment with irreversibly dependent on a life-supporting device such as a ventilator. In other instances, intensive care treatment is only survived with severe psychological and physical deficits, which can mean considerable impairment to the patient's quality of life and a significant, ongoing burden for the relatives [743].

In the face of a poor prognosis, not only for the shortterm but also the long-term, the indication for continuing long-term MV in this particular patient collective must always be consistently examined in terms of the expected quality of life of each individual patient.

The decision to alter the therapeutic goals (such as terminating ventilation therapy) can be justified by taking the ethical aspects into account and critically examining the requirements for the treatment measure to be permissible.

\subsubsection{Ethical Consideration}

A permissible treatment measure must fulfil 2 requirements:

- There is a medical indication for initiating or continuing the therapy according to the treating physicians.

- Performing the treatment corresponds to the patient's wishes

If the treatment measure under consideration meets both requirements, the treatment must be initiated or continued. If one of the 2 prerequisites is not met, a change in the therapeutic objective and the limitation of the therapy are not only permitted, but even deemed necessary [744].

A medical indication represents the professionally justified opinion that a treatment measure (e.g., prolonged weaning therapy) is suitable for achieving a specific therapeutic goal with a certain probability. Therefore, an in- dication can only be established if a therapeutic goal was previously defined [744].

In the first step, the planned measure (i.e., prolonged ventilation therapy) is examined based on as much evidence as possible in order to determine whether it is fundamentally suitable for achieving the desired therapeutic goal. This gives rise to the question of whether the illness can be successfully treated with the intended therapy.

In the second step, it is established whether the planned measure is also suitable for helping the patient in his/her particular situation. This gives rise to the question of whether a particular patient with a particular illness that has a specific degree of severity and prognosis can benefit from this therapy [744].

Indicated therapeutic measures are offered to the patient. If an indication appears doubtful, the offer is then critically and openly discussed with the patient or legal guardian. Non or contra indicated measures may neither be offered nor performed [744].

Being able to agree on the therapeutic goals is an important part of the doctor-patient relationship. In order to obtain a profile of the patient that is as comprehensive as possible, the intensive care physician not only takes medical history and findings into account, but also draws on existing knowledge and individual patient-related factors (e.g., known treatment preferences, personal values, religious beliefs, age). For patients with a doubtful prognosis, this process usually results in different therapeutic goals, which can tend to be either curative or palliative. All those involved in the treatment must jointly clarify whether the therapeutic goals can be credibly achieved. The interdisciplinarily developed therapeutic goals must now be communicated to the patient or to his/ her representative in order to enable a joint decision. All therapeutic goals should be presented and discussed in detail. The final binding decision about the desired therapeutic goals is made by the patient or his/her representative [744].

In order to be able to make a suitable decision, a supervisor is appointed as soon as possible. If a patient is unable to provide consent and therefore has a legal guardian, the legal guardian (healthcare proxy or appointed caregiver) makes the decision.

The patient's wishes are determined and documented as follows:

- The wishes stated directly by the patient

- The written advance healthcare directive of the patient

- The presumed wishes of the patient
Schönhofer et al. 


\subsubsection{The Question of the Purpose of Treatment}

During the treatment process, the question of whether the therapy has a purpose should be regularly addressed. This pertains to whether a therapeutic goal, as well as the diagnostic, therapeutic or nursing measures that depend on it, are meaningful. This question cannot be answered objectively, but requires reversion to individual and subjective assessments such as those relating to the significance of life, death and suffering, or to the quality of life, or life goals and plans. This recourse occurs both intuitively and in the form of a considered rational process [743].

Actions or states acquire a purpose by attaching significance to them in order to achieve (life) goals. Ethical action is based on the principle of "primum non nocere" - first of all, do no harm.

What appears to one person as a meaningful sustainment of life can be considered by the next as an agonising prolongation of the dying process. Examining the usefulness of a measure thus allows the result that this same measure is deemed "useful" or "pointless", depending on the point of view.

The question of appropriateness contains two components: purpose rationality and value rationality. Both can be considered and discussed separately.

Purpose rationality describes the suitability of a measure for achieving a certain goal (Example: treating an infection with antibiotics). In this respect, a medical or nursing measure makes sense if there is sufficient experience or evidence to suggest that this measure can bring about a successful treatment with a certain probability. On the contrary, a measure has value rationality if it is able to appropriately express or emphasise certain basic moral values (Example: helping a patient with an infectious disease).

Therefore, if the appropriateness of a measure has to be assessed, it is necessary to address questions such as the value of the intended therapeutic goals, the significance of suffering and illness, the subjective factors of the quality of life, and the importance of professional help or family support. The assertion that a measure is reasonable hence includes an assessment of both its rationality of purpose (it is technically reasonable) and its rationality of value (it is humanly appropriate) [743].

The following questions must be addressed when evaluating the appropriateness of treatment concepts or measures:

- Can the desired therapy goal be achieved according to professional assessment?

- Is this therapeutic goal desired by the patient?

Prolonged Weaning: S2k Guideline by the German Respiratory Society
- Are the burdens experienced during treatment justified from the patient's perspective by the quality of life/ life perspective that can be attained?

Therapy goals or concepts must therefore be questioned if:

- the desired therapy goal cannot be achieved,

- or this therapy goal is not covered in the patient's advance directive

- or the resulting quality of life/life perspective does not justify from the patient's perspective the burdens experienced during treatment

In this context, it is explicitly advised that patients who have not been successfully weaned off the ventilator (i.e., those who continue to be highly dependent on ventilation) must expressly consent - as far as it is possible - to the transition to home MV. It is therefore necessary to determine the patient's wishes in each case. On the other hand, an evaluation of the patient's wishes based on the treatment team's own values must not determine the course of action. In cases of the patient being unable to give consent, it is usually the task of the legal representative or caregiver to determine and assert the patient's wishes. If this approach does not correspond to the patient wishes, consideration must be given to ceasing MV accordingly.

It sometimes happens that the therapeutic goals deemed achievable from a medical point of view are not supported by the patient because they do not correspond to his/her idea of a good life. It is also possible that a therapeutic goal is not pursued by the patient because he/she does not want to take on the burden of the necessary treatment. In both cases the treatment appears pointless from the perspective of the patient and should therefore not be carried out.

The treatment team must be aware that beyond medical findings and criteria, there are patient-specific evaluations that call into question the appropriateness of a medically indicated therapy; these include the idea of a successful life and worthy death, the meaning of death, individual life goals, and aspects of quality of life.

In any case, before terminating life-prolonging measures based on this kind of patient self-assessment, it must be ensured that this decision is not reached during a disease-related or temporary mood state. In case of any doubt, an independent professional assessment should take place (e.g., by a psychiatrist) [743].

\subsubsection{Legal Requirements}

Regardless of the type and stage of disease, written advance healthcare directives or living wills have been le- 
gally binding in Germany since September 1st 2009, if the stipulations contained therein apply to the current living and treatment situation, and there are no indications that the person concerned has altered his/her decision in the meantime [745]. The caregiver checks whether the specifications outlined in the advance directive pertain to the current living and treatment situation; if this is the case, the caregiver then has the role of expressing and asserting the patient's wishes [745].

The treating physician determines which measure is indicated with respect to the overall condition and prognosis of the patient, and then discusses this measure with the caregiver, using the patient's wishes as a basis for the decision to be made.

If no advance directive is available, or if the details of the advance directive do not pertain to the current living and treatment situation, then the caregiver must establish the presumed wishes of the patient and then use these as the basis for his/her decision [745]. At this point, the treatment wishes expressed by the patient play an important role, while ethical and religious convictions as well as other moral concepts also need to be taken into account. Although anchored in law, current clinical practice devotes too little attention to treatment wishes that have already been expressed [745].

Once the presumed wishes of the patient have been established, close relatives and confidants of should be given the opportunity to express their views, provided this doesn't lead to considerable delay [745].

In recent decades, so-called medical paternalism has been increasingly abandoned in favour of participatory decision-making based on informed consent. Legal representatives are often overwhelmed by end-of-life decisions, as they have difficulty assessing the medical situation and may also find it challenging to take responsibility for the (presumed) wishes of the patient. Therefore, it is imperative that the legal representatives and relatives receive a highly detailed, comprehensible and honest explanation of patient's situation.

In an increasingly multicultural society, the different religious and cultural values of patients and their families must also be taken into account.

The possibility of drafting a living will is now known to almost all citizens: According to a 2014 survey conducted by a German polling institute (Demoscopy Institute, Allensbach), $28 \%$ of citizens possessed a living will [746], and this number has steadily risen since 2009. The topic of a living will becomes increasingly sensitive with age: Every second person over the age of 60 has a living will, while a further $34 \%$ plan to have one. Powers of at- torney (POAs) and living wills are also becoming increasingly common for intensive care patients. Out of the 998 patients in large German intensive care units, 512 stated that they had already created an advanced directive, i.e. 365 patients $(38.6 \%)$ a POA, 293 patients $(29.4 \%)$ a living will (the sum exceeds the total of 512 patients because some patients had a POA and living will, some only one of both). Almost $50 \%$ of the patients (486/48.7\%) had not yet drafted such a document [747].

\subsubsection{Ethical Counselling}

End-of-life therapy decisions require that the physician not only has expertise in intensive care medicine, but also competence in ethical and palliative medicine [748]. Consultations with and guidance from ethics committees are especially helpful in situations with differing perspectives [749], and can also generally contribute to improving communication during the decision process [750]. These types of committees should be composed in interdisciplinary and multi-professional fashion in order to be able to address medical, ethical, psychosocial, spiritual and legal issues.

Ethical counselling can be requested not only by the treatment team, but also by the patient, caregiver or family members [744].

During an ethical counselling session, different perspectives are presented in order to find a sustainable solution by consensus amongst all parties concerned.

An ethical counselling session always begins with an explanation of the procedure to all those involved, stressing that its outcome is a recommendation, while decisions are only to be made by those directly responsible for the patient.

The counselling focuses on the assessment of therapeutic goals, quality of life, burdens, and the chances of success. This helps to ensure that the ongoing procedure is oriented towards both what is medically feasible and the patient's individual treatment preferences, life plans and values.

In most hospitals, the qualified implementation and organization of ethical case counselling is in the hands of the hospital ethics committee, but there are also other implementation models. Since ethical counselling is a requirement for high-quality patient care, appropriate structures must be set up in all hospitals providing acute and emergency care [744].

R48: Since the medical indication and consideration of the patient's wishes are central ethical principles, it is recommended that they are taken into account for decisions pertaining to the end-of-life phase. One way of ex- 
pressing the patient's wishes is to draft a living will at an early stage.

$R$ 49: Joint discussions between the treatment team, patients and/or their legal representatives are recommended for making decisions about the end-of-life phase.

R50: Ethical case counselling is recommended for cases in which conflict exists (within the patient's family, or between the family and the treatment team) about endof-life decisions.

\subsection{Communication}

Decisions regarding medical procedures such as maximum therapy, refusal of treatment, limitation of treatment, or a change in therapy goals must be communicated in an understandable way [744].

A structured discussion with the relatives or other trusted caregivers of the patient must take place as early as possible in the course of treatment. This is mandatory for carers or authorised representatives, even if they are not relatives. This meeting with the relatives serves to provide mutual information about the patient's situation, treatment and preferences. The essential components of the discussion are as follows [744]:

- Obtaining medical and personal information about the patient

- Providing detailed information about the illness, therapy goals, and treatment, as well as the prognosis of the patient, insofar as this corresponds to the (presumed) wishes of the patient and takes medical confidentiality into account

- Support and advice for the relatives in the event of an acute crisis

The meeting should take place in a quiet room away from the noise and hectic pace of the ICU.

The treatment team should be well prepared for the meeting with the relatives, which requires a clear time plan and the determination of participants. All members of the treatment team should be represented in these discussion, especially the nursing staff, as they serve as a key intermediary. If different views about the patient's wishes or the appropriateness of ongoing medical procedures arise during the course of the discussions with the relatives, ethical case counselling is advised.

Comment: The treatment team can document the meetings using the "Therapy Limitation" form provided by the Ethics Section of the DIVI [751].

For patients on long-term MV without analgosedation, communication is often still possible, even during the periods of ventilation therapy. The doctor must inform the patient in a compassionate way about his/her prognosis and explain the current medical indication for further treatment combined with a realistic therapy goal (see above). This is the point at which the determination of the patient's wishes is of decisive importance. It is the genuine task of the physician to determine whether or not a therapeutic goal can be achieved, but only the patient or his/her representative can decide whether attainable therapy goals are actually desired [751]. In any case, it is useful to include advice from the nursing team and relatives.

Further details on the practical aspects can be found in the guidelines for family-oriented care on the neonatal, paediatric intensive-care, and adult intensive-care wards [752].

R51: A rigorous documentation process is recommended for the planning, implementation and outcome of discussions with the patient's relatives. Written documentation is suggested for recording the outcome and resulting measures of decisions to change the therapeutic objective with regard to the medical indication and the patient's wishes.

\subsubsection{Discussion Conduct}

Structured conversations with patients, family members, and care team members improve the quality of care.

The use of the expression, "withdrawal of medical therapeutic measures" needs to be replaced with "changes to therapeutic goals." The options for and importance of symptom relief for maintaining the best possible quality of life are emphasised. The important elements of good communication are:

- Appreciation for the situation

- Granting the expression of emotions

- Ability to listen

- Asking questions about the patient's life and general nature

- Encouraging relatives to ask questions

\subsection{Finishing $M V$ Treatment}

Based on the remarks above, and in accordance with the principles of the German Medical Association (Bundesärztekammer), the indication for a change in therapeutic goals in (ventilated) intensive-care patients must be promptly reviewed in the following situations:

- The patient is in the immediate process of dying

- The patient or his/her representative desire a change in therapeutic goals

- There have been significant medical changes that require the therapeutic goals to be changed

Although age alone does not represent a decisive criterion, a fragile constitution along with multimorbidity 
play an important role in the prognosis of intensive care patients [753], and hence should be taken into consideration during the decision-making process.

If the curative objective is abandoned, all diagnostic, therapeutic and nursing measures need to be reviewed. However, placing an additional burden on the dying patient by neglecting symptom-oriented measures must be avoided at all costs [744].

If it is recognized that a curative approach is no longer possible, the use of intensive care measures must be critically questioned in order not to expose patients to the risk of "over-treatment at the end of life". This is the latest point at which aspects of palliative care must be integrated into the patient's intensive care program [754]. In this context, palliative care should be seen as a complementary, expert means of treating pain and controlling symptoms such as dyspnoea, and providing respectful, caring support of the patient and his/her family in the final phase of life, as well as during the dying process [754]. A humane approach to life, death and dying, and the preservation of autonomy and respect for the dignity of seriously ill and dying patients were, and still are, the central themes of the modern hospice movement and palliative care. Palliative medical care is based on a high level of professional competence, as well as the collaboration between different professional groups, and is supplemented by volunteers [754]. For this reason, a physician with experience in palliative care should become involved in the care of the patient at an early time point.

\subsubsection{Procedures for Ending Ventilation Therapy}

Mechanical ventilation therapy can either be terminated abruptly or slowly reduced in scope (retraction of the invasive component of $\mathrm{MV}$, such as the reduction of $\mathrm{PEEP}$ or $\mathrm{FiO}_{2}$ ). The refusal of treatment or withdrawal of therapy is handled differently across intensive care units: A 2005 survey of European respiratory intermediate-care wards revealed that $21 \%$ of admitted patients with chronic respiratory failure made an end-of-life treatment decision; the mortality rate among these patients was $68 \%$, while the withdrawal of therapy was undertaken in $11 \%$ [755].

Asch et al. observed that life-sustaining therapies were frequently withdrawn in a particular order: first, the administration of blood products, haemodialysis and vasopressors was discontinued, followed by ventilation and concluded with intravenous fluids and tube feedings [756].
Based on a small case series, Campbell et al. described patients' reactions to the process of so-called terminal weaning from ventilation [748]. However, the term "terminal weaning" is essentially misleading, as it has nothing to do with the weaning process that is ultimately aimed at sufficient spontaneous breathing [757]. Hence, the more appropriate expression for this is "compassionate extubation" [758], whereby the invasive component of the ventilation is gradually reduced through the retraction of positive end-expiratory pressure or inspiratory $\mathrm{O}_{2}$ oxygen concentration, while the ventilation interface remains in place. In contrast, the process of terminal extubation involves the abrupt termination of MV and the removal of the ventilation interface. In this case, opioids or sedatives must be administered either prophylactically due to anticipated symptoms, or only after the onset of adverse symptoms [759].

Although the gradual limitation of therapy appears less intrusive and less stressful, this procedure can sometimes lead to an unjustifiable prolongation of the dying process [744].

The immediate termination of MV can lead to a significant shortening of the dying process [759]. This can elicit short-term physical reactions (such as gasping, rattle-sounding breathing; see Chapter 7.3.2). Having to observe such reactions can be stressful for the relatives and treatment team members present [744].

There are no clear, evidence-based recommendations for the decision-making process associated with the discontinuation of therapy in ventilated patients. While both terminal extubation and leaving the tube in place are possible, the former is rarely practiced in Europe. The decision for the respective procedure should be based on timely agreement within the treatment team, and with the relatives and (if possible) patient [760].

\subsubsection{Terminal Respiratory Secretions ("Death}

Rattle")

After ending MV, rattling sounds may occur during breathing (the so-called "death rattle") due to an increase in pharyngotracheal secretion formation or (terminal) pulmonary edema. This rattling is a sign of the dying process and does not affect patients, who are in a state of reduced consciousness, as displayed by the fading of defensive reflexes such as coughing and gagging. However, the acoustic phenomenon of rattling can present as an enormous psychological burden for the patient's relatives. This burden can often be reduced if the relatives are sufficiently informed about the cause of these prefinal rattling noises. In addition, this particular symptom should, 
where possible, be relieved with pharmacological intervention [761]. Intravenous fluids should initially be avoided in order to minimise secretions.

Suctioning manoeuvres that only reach the pharynx or trachea should be performed in combination with anticholinergic drugs such as hyoscine butylbromide or glycopyrronium bromide.

R52: To prevent stress and suffering from respiratory distress, anxiolysis and sedation is recommended to be carried out in individually-adjusted doses and precisely documented. A premature death due to unavoidable sideeffects can be accepted.

\subsection{Documentation}

A form for documenting decisions to withhold or withdraw therapy is now available [751]. Standardised documentation of the decisions made at the end of life, along with visibility in the patient chart, are essential for transparent and comprehensible treatment, even from a medicolegal perspective. Agreements in and changes to a patient's therapeutic goals must be made known to all treatment sectors within the hospital, since they have to be observed by the entire treatment team. Documentation of the agreements reached is easily manageable and not only ensures that there is clarity amongst the different wards within a hospital, it also prevents the introduction or continuation of measures that are not/no longer indicated, or not/no longer desired. For these purposes, a standardised documentation form for the withholding/ withdrawing of therapy should be used. The documented decisions should be the result of interdisciplinary discussions. The significant reasons for these decisions should be clearly and comprehensibly documented in the patient's medical records. Documentation of the structured communication that has taken place with the patient and relatives, as described in the DIVI quality indications, can serve as a helpful basis for reaching a decision [762]. The documentation form summarizes this decisionmaking process in a clear and concise manner and stores it in a central location. It serves as a reliable basis for decision-making, provided there are no grounds for possible changes. In this respect, however, it does not absolve the treatment team from the responsibility of making decisions in the specific situation. Adapting the document to the local circumstances of the treating hospital is recommended in order to clearly and comprehensibly record (on paper and/or electronically) the decisions made. It should be part of the patient's file and accompany the patient through all functional sections of the hospital.

Prolonged Weaning: S2k Guideline by the German Respiratory Society
R53: A standardised form is recommended for documenting all decisions relating to the withholding/withdrawal of therapy. Documentation of the significant reasons for these decisions in the patient's medical files is also recommended.

QI5: Number of standardised documentations of decisions regarding the withholding/withdrawal of therapy/ Number of decisions regarding the withholding/withdrawal of therapy in prolonged weaning

\subsection{Special Aspects in Paediatric Patients}

In Germany, the withholding or withdrawal of therapy in infants and children is necessary and currently ethically accepted in two situations: (1) If treatment is futile, its continuation only means prolongation of suffering. Futile here means only a short gain in life span, which is not in proportion to the suffering the patient has to endure. (2) In case of survival, the child's quality of life is considered to be that of unacceptable suffering, without any possibility of improvement $[763,764]$. Therapy withdrawal more often takes place on the basis that treatment is futile [765]. Even severe mental or physical disabilities or home MV need not significantly impair a child's subjective quality of life [766-769]. The prognosis of an unendurable quality of life is therefore difficult to predict and this particular status can even eventuate slowly in the ambulatory course of the disease; for example, in progressive neurodegenerative or even neuromuscular diseases. In these cases, it is the task of the physician to ascertain whether it is living or dying that is in the best interest of the child (some lawyers also refer to the presumed will of the child). The necessity for treatment limitation in childhood should be determined by formal ethical consultation. The outcome is discussed with the parents/legal guardians, who are also informed about all other treatment options and consequences. The decision about the intensity of ongoing therapy is made in conjunction with the parents/legal guardians [763, 770-773].

Parents require different amounts of time to understand and accept the situation, and to be able to support a decision to withhold or withdraw therapy [773]. Close guidance without pressure, as well as psychological support for the parents are required. Most parents prefer to be present when therapy is terminated and potentially hold the child in their arms. The use of analgesics or sedatives is intended to prevent patient suffering as much as possible [774].

R54: If therapy appears futile, or if in cases of survival there is unreasonable suffering without the possibility of improvement, it is recommended to determine whether it is living or dying that is in the best interest of the child. 
R55: Decisions about the continuation or withdrawal of treatment are suggested to be made in the framework of an ethical consultation, and with the agreement of the parents.

\section{Acknowledgements}

We thank Dr. Sandra Dieni for her assistance in translating the manuscript from the German language in English and Dr. Nina Hämäläinen (Institute for Lung Research, Berlin) for organising the consensus conferences and providing the literature service.

\section{Statement of Ethics}

There are no ethical issues associated to this Guideline.

\section{Conflict of Interest Statement}

Authors have no conflict of interest. An overview of the conflicts of interest can be found online at http://awmf.org; AWMFRegistration Number: 020-015.

\section{Funding Sources}

The German Respiratory Society (DGP) financed the three consensus conferences of the Guideline process and the translation from German into English.

\section{Author Contributions}

B. Schönhofer: Head of the guideline committee; Main topics: Weaning strategies and therapy decisions for the end-of-life phase. J. Geiseler: Main topics: Interfaces and Physiotherapy (Weaning Strategies). D. Dellweg: Main topics: Ventilation modes and Rehabilitation. H. Fuchs: Main topic: Special Characteristic of Paediatric Patients. O. Moerer: Main topics: Ventilation modes and blood transfusion. S. Weber-Carstens: Main topics: Infrastructure and process organisation for the care of patients in prolonged weaning and protocols. M. Westhoff: Main topics: Pathophysiology of Weaning Failure and NIV. W. Windisch: Main topics: Definitions, Epidemiology, and Weaning Categories.

\section{References}

1 Ibsen B. The anaesthetist's viewpoint on the treatment of respiratory complications in poliomyelitis during the epidemic in Copenhagen, 1952. Proc R Soc Med. 1954 Jan;47(1): 72-4.

2 Reisner-Sénélar L. The birth of intensive care medicine: björn Ibsen's records. Intensive Care Med. 2011 Jul;37(7):1084-6.

3 Windisch W, Dreher M, Geiseler J, Siemon K, Brambring J, Dellweg D, et al.; für die Leitliniengruppe „Nichtinvasive und invasive Beatmung als Therapie der chronischen respiratorischen Insuffizienz". [Guidelines for Non-Invasive and Invasive Home Mechanical Ventilation for Treatment of Chronic Respiratory Failure - Update 2017]. Pneumologie. 2017 Nov;71(11):722-95.

4 Esteban A, Anzueto A, Frutos F, Alía I, Brochard L, Stewart TE, et al.; Mechanical Ventilation International Study Group. Characteristics and outcomes in adult patients receiving mechanical ventilation: a 28-day international study. JAMA. 2002 Jan;287(3):345-55.

5 Goligher E, Ferguson ND. Mechanical ventilation: epidemiological insights into current practices. Curr Opin Crit Care. 2009 Feb; 15(1):44-51.

6 Esteban A, Alía I, Ibañez J, Benito S, Tobin MJ; The Spanish Lung Failure Collaborative Group. Modes of mechanical ventilation and weaning. A national survey of Spanish hospitals. Chest. 1994 Oct;106(4):1188-93.
7 Ely EW, Baker AM, Dunagan DP, Burke HL, Smith AC, Kelly PT, et al. Effect on the duration of mechanical ventilation of identifying patients capable of breathing spontaneously. N Engl J Med. 1996 Dec;335(25):1864-9.

8 Kollef MH, Shapiro SD, Silver P, St John RE, Prentice D, Sauer S, et al. A randomized, controlled trial of protocol-directed versus physician-directed weaning from mechanical ventilation. Crit Care Med. 1997 Apr;25(4):567-74.

9 Cohen IL, Booth FV. Cost containment and mechanical ventilation in the United States. New Horiz. 1994 Aug;2(3):283-90.

10 Huttmann SE, Windisch W, Storre JH. Invasive home mechanical ventilation: living conditions and health-related quality of life. Respiration. 2015;89(4):312-21.

11 Huttmann SE, Magnet FS, Karagiannidis C, Storre JH, Windisch W. Quality of life and life satisfaction are severely impaired in patients with long-term invasive ventilation following ICU treatment and unsuccessful weaning. Ann Intensive Care. 2018 Mar;8(1):38.

12 Schönhofer B, Euteneuer S, Nava S, Suchi S, Köhler D. Survival of mechanically ventilated patients admitted to a specialised weaning centre. Intensive Care Med. 2002 Jul;28(7): 908-16.

13 Schönhofer B, Geiseler J, Dellweg D, Moerer $\mathrm{O}$, Barchfeld T, Fuchs $\mathrm{H}$, et al. [Prolonged weaning: S2k-guideline published by the German Respiratory Society]. Pneumologie. 2014 Jan;68(1):19-75.
14 https://www.bsg.bund.de/DE/Entscheidungen/entscheidungen_node.html.

15 www.awmf.org/leitlinien/aktuelle-leitlinien.

16 Westhoff M, Schönhofer B, Neumann P, Bickenbach J, Barchfeld T, Becker H, et al. [Noninvasive Mechanical Ventilation in Acute Respiratory Failure]. Pneumologie. 2015 Dec;69(12):719-56.

17 Boles JM, Bion J, Connors A, Herridge M, Marsh B, Melot C, et al. Weaning from mechanical ventilation. Eur Respir J. 2007 May; 29(5):1033-56.

18 Torres A, Gatell JM, Aznar E, el-Ebiary M, Puig de la Bellacasa J, González J, et al. Reintubation increases the risk of nosocomial pneumonia in patients needing mechanical ventilation. Am J Respir Crit Care Med. 1995 Jul;152(1):137-41.

19 Epstein SK, Ciubotaru RL, Wong JB. Effect of failed extubation on the outcome of mechanical ventilation. Chest. 1997 Jul;112(1):18692.

20 Seymour CW, Martinez A, Christie JD, Fuchs BD. The outcome of extubation failure in a community hospital intensive care unit: a cohort study. Crit Care. 2004 Oct;8(5):R322-7.

21 Coplin WM, Pierson DJ, Cooley KD, Newell DW, Rubenfeld GD. Implications of extubation delay in brain-injured patients meeting standard weaning criteria. Am J Respir Crit Care Med. 2000 May;161(5):1530-6. 
22 Esteban A, Alía I, Tobin MJ, Gil A, Gordo F, Vallverdú I, et al.; Spanish Lung Failure Collaborative Group. Effect of spontaneous breathing trial duration on outcome of attempts to discontinue mechanical ventilation. Am J Respir Crit Care Med. 1999 Feb;159(2): 512-8.

23 Vallverdú I, Calaf N, Subirana M, Net A, Benito S, Mancebo J. Clinical characteristics, respiratory functional parameters, and outcome of a two-hour T-piece trial in patients weaning from mechanical ventilation. Am J Respir Crit Care Med. 1998 Dec;158(6):185562.

24 Epstein SK, Nevins ML, Chung J. Effect of unplanned extubation on outcome of mechanical ventilation. Am J Respir Crit Care Med. 2000 Jun;161(6):1912-6.

25 Epstein SK. Decision to extubate. Intensive Care Med. 2002 May;28(5):535-46.

26 Lemaire F. Difficult weaning. Intensive Care Med. 1993;19(Suppl 2):S69-73.

27 Epstein SK, Durbin CG Jr. Should a patient be extubated and placed on noninvasive ventilation after failing a spontaneous breathing trial? Respir Care. 2010 Feb;55(2):198-206.

28 Nava S, Ambrosino N, Clini E, Prato M, Orlando $G$, Vitacca $M$, et al. Noninvasive mechanical ventilation in the weaning of patients with respiratory failure due to chronic obstructive pulmonary disease. A randomized, controlled trial. Ann Intern Med. 1998 May; 128(9):721-8.

29 Esteban A, Frutos-Vivar F, Ferguson ND, Arabi Y, Apezteguía C, González M, et al. Noninvasive positive-pressure ventilation for respiratory failure after extubation. $\mathrm{N}$ Engl J Med. 2004 Jun;350(24):2452-60.

30 Nava S, Gregoretti C, Fanfulla F, Squadrone E, Grassi M, Carlucci A, et al. Noninvasive ventilation to prevent respiratory failure after extubation in high-risk patients. Crit Care Med. 2005 Nov;33(11):2465-70.

31 Ferrer M, Valencia M, Nicolas JM, Bernadich O, Badia JR, Torres A. Early noninvasive ventilation averts extubation failure in patients at risk: a randomized trial. Am J Respir Crit Care Med. 2006 Jan;173(2):164-70.

32 Funk GC, Anders S, Breyer MK, Burghuber OC, Edelmann G, Heindl W, et al. Incidence and outcome of weaning from mechanical ventilation according to new categories. Eur Respir J. 2010 Jan;35(1):88-94.

33 Schönhofer B. Beatmungsmedizin - das Wichtigste aus 4 aktuellen Leitlinien. Dtsch Med Wochenschr. 2018 Jun;143(11):793-6.

34 Béduneau G, Pham T, Schortgen F, Piquilloud L, Zogheib E, Jonas M, et al.; WIND (Weaning according to a New Definition) Study Group and the REVA (Réseau Européen de Recherche en Ventilation Artificielle) Network $\ddagger$. Epidemiology of Weaning Outcome according to a New Definition. The WIND Study. Am J Respir Crit Care Med. 2017 Mar;195(6):772-83.
35 Esteban A, Frutos F, Tobin MJ, Alía I, Solsona JF, Valverdú I, et al.; Spanish Lung Failure Collaborative Group. A comparison of four methods of weaning patients from mechanical ventilation. N Engl J Med. 1995 Feb; 332(6):345-50.

36 Carson SS, Garrett J, Hanson LC, Lanier J, Govert J, Brake MC, et al. A prognostic model for one-year mortality in patients requiring prolonged mechanical ventilation. Crit Care Med. 2008 Jul;36(7):2061-9.

37 Magnet FS, Bleichroth H, Huttmann SE, Callegari J, Schwarz SB, Schmoor C, et al. Clinical evidence for respiratory insufficiency type II predicts weaning failure in long-term ventilated, tracheotomised patients: a retrospective analysis. J Intensive Care. 2018 Oct;6(1): 67.

38 WeanNet Study Group. (WeanNet: The network of weaning units of the DGP [Deutsche Gesellschaft für Pneumologie und Beatmungsmedizin] - results to epidemiology an outcome in patients with prolonged weaning). Dtsch Med Wochenschr 2016; 141: e166-72.

39 Karagiannidis C, Strassmann S, Callegari J, Kochanek M, Janssens U, Windisch W. Epidemiologische Entwicklung der außerklinischen Beatmung: eine rasant zunehmende Herausforderung für die ambulante und stationäre Patientenversorgung. Dtsch $\mathrm{Med}$ Wochenschr. 2019 May;144(9):e58-63.

40 Bellemare F, Grassino A. Effect of pressure and timing of contraction on human diaphragm fatigue. J Appl Physiol. 1982 Nov; 53(5):1190-5

41 Jubran A, Tobin MJ. Passive mechanics of lung and chest wall in patients who failed or succeeded in trials of weaning. Am J Respir Crit Care Med. 1997 Mar;155(3):916-21.

42 Tobin MJ, Laghi F. Monitoring of Respiratory Muscle Function. In: Tobin MJ, editor. Principle and practice of intensive care monitoring. New York: McGraw-Hill; 1998.

43 Vassilakopoulos T, Zakynthinos S, Roussos C. Respiratory muscles and weaning failure. Eur Respir J. 1996 Nov;9(11):2383-400.

44 Lopata M, Onal E. Mass loading, sleep apnea, and the pathogenesis of obesity hypoventilation. Am Rev Respir Dis. 1982 Oct;126(4): 640-5.

45 MacIntyre NR, Cook DJ, Ely EW Jr, Epstein SK, Fink JB, Heffner JE, et al.; American College of Chest Physicians; American Association for Respiratory Care; American College of Critical Care Medicine. Evidence-based guidelines for weaning and discontinuing ventilatory support: a collective task force facilitated by the American College of Chest Physicians; the American Association for Respiratory Care; and the American College of Critical Care Medicine. Chest. 2001 Dec; 120(6 Suppl):375S-95S.

46 Raju P, Manthous CA. The pathogenesis of respiratory failure: an overview. Respir Care Clin N Am. 2000 Jun;6(2):195-212.
47 Fishman AP, Turino GM, Bergofsky EH. The syndrome of alveolar hypoventilation. Am J Med. 1957 Sep;23(3):333-9.

48 Vallverdú I, Mancebo J. Approach to patients who fail initial weaning trials. Respir Care Clin N Am. 2000 Sep;6(3):365-84.

49 Barrientos-Vega R, Mar Sánchez-Soria M, Morales-García C, Robas-Gómez A, CuenaBoy R, Ayensa-Rincon A. Prolonged sedation of critically ill patients with midazolam or propofol: impact on weaning and costs. Crit Care Med. 1997 Jan;25(1):33-40.

50 Wheeler AP. Sedation, analgesia, and paralysis in the intensive care unit. Chest. 1993 Aug; 104(2):566-77.

51 Spitzer AR, Giancarlo T, Maher L, Awerbuch G, Bowles A. Neuromuscular causes of prolonged ventilator dependency. Muscle Nerve. 1992 Jun;15(6):682-6.

52 De Jonghe B, Bastuji-Garin S, Sharshar T, Outin H, Brochard L. Does ICU-acquired paresis lengthen weaning from mechanical ventilation? Intensive Care Med. 2004 Jun;30(6): 1117-21.

53 Hermans G, De Jonghe B, Bruyninckx F, Van den Berghe G. Clinical review: critical illness polyneuropathy and myopathy. Crit Care. 2008;12(6):238.

54 Maher J, Rutledge F, Remtulla H, Parkes A, Bernardi L, Bolton CF. Neuromuscular disorders associated with failure to wean from the ventilator. Intensive Care Med. 1995 Sep; 21(9):737-43.

55 Peterson WP, Whiteneck GG, Gerhart KA. Chest tubes, lung entrapment, and failure to wean from the ventilator. Report of three patients with quadriplegia. Chest. 1994 Apr; 105(4):1292-4.

56 Garnacho-Montero J, Madrazo-Osuna J, García-Garmendia JL, Ortiz-Leyba C, Jiménez-Jiménez FJ, Barrero-Almodóvar A, et al. Critical illness polyneuropathy: risk factors and clinical consequences. A cohort study in septic patients. Intensive Care Med. 2001 Aug;27(8):1288-96.

57 Bercker S, Weber-Carstens S, Deja M, Grimm C, Wolf S, Behse F, et al. Critical illness polyneuropathy and myopathy in patients with acute respiratory distress syndrome. Crit Care Med. 2005 Apr;33(4):711-5.

58 Garnacho-Montero J, Amaya-Villar R, García-Garmendía JL, Madrazo-Osuna J, OrtizLeyba C. Effect of critical illness polyneuropathy on the withdrawal from mechanical ventilation and the length of stay in septic patients. Crit Care Med. 2005 Feb;33(2):34954.

59 Schweickert WD, Hall J. ICU-acquired weakness. Chest. 2007 May;131(5):1541-9.

60 Puthucheary Z, Rawal J, Ratnayake G, Harridge $\mathrm{S}$, Montgomery $\mathrm{H}$, Hart N. Neuromuscular blockade and skeletal muscle weakness in critically ill patients: time to rethink the evidence? Am J Respir Crit Care Med. 2012 May;185(9):911-7. 
61 Alhazzani W, Alshahrani M, Jaeschke R, Forel JM, Papazian L, Sevransky J, et al. Neuromuscular blocking agents in acute respiratory distress syndrome: a systematic review and meta-analysis of randomized controlled trials. Crit Care. 2013 Mar;17(2):R43.

62 Apostolakis E, Papakonstantinou NA, Baikoussis NG, Papadopoulos G. Intensive care unit-related generalized neuromuscular weakness due to critical illness polyneuropathy/myopathy in critically ill patients. J Anesth. 2015 Feb;29(1):112-21.

63 Annane D. What Is the Evidence for Harm of Neuromuscular Blockade and Corticosteroid Use in the Intensive Care Unit? Semin Respir Crit Care Med. 2016 Feb;37(1):51-6.

64 Vassilakopoulos T, Petrof BJ. Ventilator-induced diaphragmatic dysfunction. Am J Respir Crit Care Med. 2004 Feb;169(3):33641.

65 Jubran A. Critical illness and mechanical ventilation: effects on the diaphragm. Respir Care. 2006 Sep;51(9):1054-61.

66 Kabitz HJ, Windisch W, Schönhofer B. Ventilator induzierter Zwerchfellschaden: ein Update. Pneumologie. 2013 Aug;67(8):43541.

67 Hermans G, Van Mechelen H, Clerckx B, Vanhullebusch T, Mesotten D, Wilmer A, et al. Acute outcomes and 1-year mortality of intensive care unit-acquired weakness. A cohort study and propensity-matched analysis. Am J Respir Crit Care Med. 2014 Aug;190(4):41020.

68 Gay PC, Rodarte JR, Hubmayr RD. The effects of positive expiratory pressure on isovolume flow and dynamic hyperinflation in patients receiving mechanical ventilation. Am Rev Respir Dis. 1989 Mar;139(3):621-6.

69 Parthasarathy S, Jubran A, Tobin MJ. Cycling of inspiratory and expiratory muscle groups with the ventilator in airflow limitation. Am J Respir Crit Care Med. 1998 Nov;158(5 Pt 1): 1471-8.

70 Lim CK, Ruan SY, Lin FC, Wu CL, Chang HT, Jerng JS, et al. Effect of Tracheostomy on Weaning Parameters in Difficult-to-Wean Mechanically Ventilated Patients: A Prospective Observational Study. PLoS One. 2015 Sep;10(9):e0138294.

71 Davis K Jr, Campbell RS, Johannigman JA, Valente JF, Branson RD. Changes in respiratory mechanics after tracheostomy. Arch Surg. 1999 Jan;134(1):59-62.

72 Diehl JL, El Atrous S, Touchard D, Lemaire F, Brochard L. Changes in the work of breathing induced by tracheotomy in ventilator-dependent patients. Am J Respir Crit Care Med. 1999 Feb;159(2):383-8.

73 Moscovici da Cruz V, Demarzo SE, Sobrinho JB, Amato MB, Kowalski LP, Deheinzelin D. Effects of tracheotomy on respiratory mechanics in spontaneously breathing patients. Eur Respir J. 2002 Jul;20(1):112-7.
74 Salam A, Tilluckdharry L, AmoatengAdjepong Y, Manthous CA. Neurologic status, cough, secretions and extubation outcomes. Intensive Care Med. 2004 Jul;30(7): 1334-9.

75 Epstein SK, Ciubotaru RL. Independent ef fects of etiology of failure and time to reintubation on outcome for patients failing extubation. Am J Respir Crit Care Med. 1998 Aug; 158(2):489-93.

76 Straus C, Louis B, Isabey D, Lemaire F, Harf A, Brochard L. Contribution of the endotracheal tube and the upper airway to breathing workload. Am J Respir Crit Care Med. 1998 Jan;157(1):23-30.

77 Rumbak MJ, Walsh FW, Anderson WM, Rolfe MW, Solomon DA. Significant tracheal obstruction causing failure to wean in patients requiring prolonged mechanical ventilation: a forgotten complication of long-term mechanical ventilation. Chest. 1999 Apr; 115(4):1092-5.

78 Maeda H, Nakahara K, Ohno K, Kido T, Ikeda M, Kawashima Y. Diaphragm function after pulmonary resection. Relationship to postoperative respiratory failure. Am Rev Respir Dis. 1988 Mar;137(3):678-81.

79 Takeda S, Miyoshi S, Maeda H, Minami M, Yoon HE, Tanaka H, et al. Ventilatory muscle recruitment and work of breathing in patients with respiratory failure after thoracic surgery. Eur J Cardiothorac Surg. 1999 Apr;15(4): 449-55.

80 Gaissert H, Wilcox SR. Diaphragmatic Dysfunction after Thoracic Operations. Thorac Cardiovasc Surg. 2016 Dec;64(8):621-30.

81 Fintelmann FJ, Troschel FM, Mario J, Chretien YR, Knoll SJ, Muniappan A, et al. Thoracic Skeletal Muscle Is Associated With Adverse Outcomes After Lobectomy for Lung Cancer. Ann Thorac Surg. 2018 May; 105(5): 1507-15.

82 Richter Larsen K, Svendsen UG, Milman N, Brenøe J, Petersen BN. Exercise testing in the preoperative evaluation of patients with bronchogenic carcinoma. Eur Respir J. 1997 Jul; 10(7):1559-65.

83 Brutsche MH, Spiliopoulos A, Bolliger CT, Licker M, Frey JG, Tschopp JM. Exercise capacity and extent of resection as predictors of surgical risk in lung cancer. Eur Respir J. 2000 May;15(5):828-32.

84 Lemaire F, Teboul JL, Cinotti L, Giotto G, Abrouk F, Steg G, et al. Acute left ventricular dysfunction during unsuccessful weaning from mechanical ventilation. Anesthesiology. 1988 Aug;69(2):171-9.

85 Epstein SK. Etiology of extubation failure and the predictive value of the rapid shallow breathing index. Am J Respir Crit Care Med. 1995 Aug;152(2):545-9.

86 Richard C, Teboul JL, Archambaud F, Hebert JL, Michaut P, Auzepy P. Left ventricular function during weaning of patients with chronic obstructive pulmonary disease. Intensive Care Med. 1994;20(3):181-6.
87 Pinsky MR. Cardiovascular issues in respiratory care. Chest. 2005 Nov;128(5 Suppl 2): 592S-7S.

88 Buda AJ, Pinsky MR, Ingels NB Jr, Daughters GT 2nd, Stinson EB, Alderman EL. Effect of intrathoracic pressure on left ventricular performance. N Engl J Med. 1979 Aug;301(9): 453-9.

89 Dres M, Teboul JL, Monnet X. Weaning the cardiac patient from mechanical ventilation. Curr Opin Crit Care. 2014 Oct;20(5):493-8.

90 Jubran A, Mathru M, Dries D, Tobin MJ. Continuous recordings of mixed venous oxygen saturation during weaning from mechanical ventilation and the ramifications thereof. Am J Respir Crit Care Med. 1998 Dec;158(6): $1763-9$.

91 Vizza CD, Lynch JP, Ochoa LL, Richardson G, Trulock EP. Right and left ventricular dysfunction in patients with severe pulmonary disease. Chest. 1998 Mar;113(3):576-83.

92 Alpert JS. Effect of right ventricular dysfunction on left ventricular function. Adv Cardiol. 1986;34:25-34.

93 Tseng YH, Ko HK, Tseng YC, Lin YH, Kou YR. Atrial Fibrillation on Intensive Care Unit Admission Independently Increases the Risk of Weaning Failure in Nonheart Failure Mechanically Ventilated Patients in a Medical Intensive Care Unit: A Retrospective CaseControl Study. Medicine (Baltimore). 2016 May;95(20):e3744.

94 Gerbaud E, Erickson M, Grenouillet-Delacre M, Beauvieux MC, Coste P, Durrieu-Jaïs C, et al. Echocardiographic evaluation and $\mathrm{N}$-terminal pro-brain natriuretic peptide measurement of patients hospitalized for heart failure during weaning from mechanical ventilation. Minerva Anestesiol. 2012 Apr;78(4):415-25.

95 Moschietto S, Doyen D, Grech L, Dellamonica J, Hyvernat H, Bernardin G. Transthoracic Echocardiography with Doppler Tissue Imaging predicts weaning failure from mechanical ventilation: evolution of the left ventricle relaxation rate during a spontaneous breathing trial is the key factor in weaning outcome. Crit Care. 2012 May;16(3):R81.

96 Papanikolaou J, Makris D, Saranteas T, Karakitsos D, Zintzaras E, Karabinis A, et al. New insights into weaning from mechanical ventilation: left ventricular diastolic dysfunction is a key player. Intensive Care Med. 2011 Dec; 37(12):1976-85.

97 Voga G. Hemodynamic changes during weaning: can we assess and predict cardiac-related weaning failure by transthoracic echocardiography? Crit Care. 2010;14(4):174.

98 Richard C, Monnet X, Teboul JL. Pulmonary artery catheter monitoring in 2011. Curr Opin Crit Care. 2011 Jun;17(3):296-302. 
99 Ponikowski P, Voors AA, Anker SD, Bueno H, Cleland JG, Coats AJ, et al.; Authors/Task Force Members; Document Reviewers. 2016 ESC Guidelines for the diagnosis and treatment of acute and chronic heart failure: the Task Force for the diagnosis and treatment of acute and chronic heart failure of the $\mathrm{Eu}$ ropean Society of Cardiology (ESC). Developed with the special contribution of the Heart Failure Association (HFA) of the ESC. Eur J Heart Fail. 2016 Aug;18(8):891-975.

100 Hubmayr RD, Loosbrock LM, Gillespie DJ, Rodarte JR. Oxygen uptake during weaning from mechanical ventilation. Chest. 1988 Dec;94(6):1148-55.

101 Tassaux D, Gainnier M, Battisti A, Jolliet P. Impact of expiratory trigger setting on delayed cycling and inspiratory muscle workload. Am J Respir Crit Care Med. 2005 Nov; 172(10):1283-9.

102 Chao DC, Scheinhorn DJ, Stearn-Hassenpflug M. Patient-ventilator trigger asynchrony in prolonged mechanical ventilation. Chest. 1997 Dec;112(6):1592-9.

103 Lai YC, Ruan SY, Huang CT, Kuo PH, Yu CJ. Hemoglobin levels and weaning outcome of mechanical ventilation in difficult-to-wean patients: a retrospective cohort study. PLoS One. 2013 Aug;8(8):e73743.

104 Schönhofer B, Wenzel M, Geibel M, Köhler D. Blood transfusion and lung function in chronically anemic patients with severe chronic obstructive pulmonary disease. Crit Care Med. 1998 Nov;26(11):1824-8.

105 Silver MR. Anemia in the long-term ventilator-dependent patient with respiratory failure. Chest. 2005 Nov;128(5 Suppl 2):568S$75 S$.

106 Z'Graggen WJ, Lin CS, Howard RS, Beale RJ, Bostock H. Nerve excitability changes in critical illness polyneuropathy. Brain. 2006 Sep;129(Pt 9):2461-70.

107 Latronico N, Bolton CF. Critical illness polyneuropathy and myopathy: a major cause of muscle weakness and paralysis. Lancet Neurol. 2011 Oct;10(10):931-41.

108 Visser LH. Critical illness polyneuropathy and myopathy: clinical features, risk factors and prognosis. Eur J Neurol. 2006 Nov; 13(11):1203-12.

109 Zochodne DW, Bolton CF, Wells GA, Gilbert JJ, Hahn AF, Brown JD, et al. Critical illness polyneuropathy. A complication of sepsis and multiple organ failure. Brain. 1987 Aug;110(Pt 4):819-41.

110 Judemann K, Lunz D, Zausig YA, Graf BM, Zink W. [Intensive care unit-acquired weakness in the critically ill : critical illness polyneuropathy and critical illness myopathy]. Anaesthesist. 2011 Oct;60(10):887-901.

111 Stevens RD, Dowdy DW, Michaels RK, Mendez-Tellez PA, Pronovost PJ, Needham DM. Neuromuscular dysfunction acquired in critical illness: a systematic review. Intensive Care Med. 2007 Nov;33(11):1876-91.
112 Schmidt SB, Rollnik JD. Critical illness polyneuropathy (CIP) in neurological early rehabilitation: clinical and neurophysiological features. BMC Neurol. 2016 Dec;16(1):256.

113 Latronico N, Bertolini G, Guarneri B, Botteri M, Peli E, Andreoletti S, et al. Simplified electrophysiological evaluation of peripheral nerves in critically ill patients: the Italian multi-centre CRIMYNE study. Crit Care. 2007;11(1):R11.

114 Latronico N, Nattino G, Guarneri B, Fagoni N, Amantini A, Bertolini G; GiVITI Study Investigators. Validation of the peroneal nerve test to diagnose critical illness polyneuropathy and myopathy in the intensive care unit: the multicentre Italian CRIMYNE-2 diagnostic accuracy study. F1000 Res. 2014 Jun;3:127.

115 Moss M, Yang M, Macht M, Sottile P, Gray L, McNulty M, et al. Screening for critical illness polyneuromyopathy with single nerve conduction studies. Intensive Care Med. 2014 May;40(5):683-90.

116 Koch S, Wollersheim T, Bierbrauer J, Haas K, Mörgeli R, Deja M, et al. Long-term recovery In critical illness myopathy is complete, contrary to polyneuropathy. Muscle Nerve. 2014 Sep;50(3):431-6.

117 Thabet Mahmoud A, Tawfik MA, Abd El Naby SA, Abo El Fotoh WM, Saleh NY, Abd El Hady NM. Neurophysiological study of critical illness polyneuropathy and myopathy in mechanically ventilated children; additional aspects in paediatric critical illness comorbidities. Eur J Neurol. 2018 Jul;25(7):991-e76.

118 DeVita MA, Spierer-Rundback L. Swallowing disorders in patients with prolonged orotracheal intubation or tracheostomy tubes. Crit Care Med. 1990 Dec;18(12): 1328-30.

119 Ponfick M, Linden R, Nowak DA. Dysphagia-a common, transient symptom in critical illness polyneuropathy: a fiberoptic endoscopic evaluation of swallowing study*. Crit Care Med. 2015 Feb;43(2):365-72.

120 Dres M, Dubé BP, Mayaux J, Delemazure J, Reuter D, Brochard L, et al. Coexistence and Impact of Limb Muscle and Diaphragm Weakness at Time of Liberation from Mechanical Ventilation in Medical Intensive Care Unit Patients. Am J Respir Crit Care Med. 2017 Jan;195(1):57-66.

121 Li S, An YZ, Ren JY, Zhu FX, Chen H. Myocardial injury after surgery is a risk factor for weaning failure from mechanical ventilation in critical patients undergoing major abdominal surgery. PLoS One. 2014 Nov; 9(11):e113410.

122 Rialp Cervera G, Raurich Puigdevall JM, Morán Chorro I, Martín Delgado MC, Heras la Calle G, Mas Serra A, et al. Effects of early administration of acetazolamide on the duration of mechanical ventilation in patients with chronic obstructive pulmonary disease or obesity-hypoventilation syndrome with metabolic alkalosis. A randomized trial. Pulm Pharmacol Ther. 2017 Jun;44:30-7.
123 Oppersma E, Doorduin J, van der Hoeven JG, Veltink PH, van Hees HW, Heunks LM. The effect of metabolic alkalosis on the ventilatory response in healthy subjects. Respir Physiol Neurobiol. 2018 Feb;249:47-53.

124 Terzano C, Di Stefano F, Conti V, Di Nicola M, Paone G, Petroianni A, et al. Mixed acidbase disorders, hydroelectrolyte imbalance and lactate production in hypercapnic respiratory failure: the role of noninvasive ventilation. PLoS One. 2012;7(4):e35245.

125 Sauthier M, Rose L, Jouvet P. Pediatric Prolonged Mechanical Ventilation: Considerations for Definitional Criteria. Respir Care. 2017 Jan;62(1):49-53.

126 Baisch SD, Wheeler WB, Kurachek SC, Cornfield DN. Extubation failure in pediatric intensive care incidence and outcomes. Pediatr Crit Care Med. 2005 May;6(3):3128.

127 Graham RJ, Fleegler EW, Robinson WM. Chronic ventilator need in the community: a 2005 pediatric census of Massachusetts. Pediatrics. 2007 Jun;119(6):e1280-7.

128 Jardine E, O’Toole M, Paton JY, Wallis C. Current status of long term ventilation of children in the United Kingdom: questionnaire survey. BMJ. 1999 Jan;318(7179):2959.

129 Kurachek SC, Newth CJ, Quasney MW, Rice T, Sachdeva RC, Patel NR, et al. Extubation failure in pediatric intensive care: a multiplecenter study of risk factors and outcomes. Crit Care Med. 2003 Nov;31(11):2657-64.

130 Joho-Arreola AL, Bauersfeld U, Stauffer UG, Baenziger O, Bernet V. Incidence and treatment of diaphragmatic paralysis after cardiac surgery in children. Eur J Cardiothorac Surg. 2005 Jan;27(1):53-7.

131 Teoh HL, Mohammad SS, Britton PN, Kandula T, Lorentzos MS, Booy R, et al. Clinical Characteristics and Functional Motor Outcomes of Enterovirus 71 Neurological Disease in Children. JAMA Neurol. 2016 Mar; 73(3):300-7.

132 Nicolai T. Therapeutic concepts in upper airway obstruction. Paediatr Respir Rev. 2004 Mar;5(1):34-9.

133 Corbett HJ, Mann KS, Mitra I, Jesudason EC, Losty PD, Clarke RW. Tracheostomy-a 10 -year experience from a UK pediatric surgical center. J Pediatr Surg. 2007 Jul;42(7): 1251-4.

134 Fontela PS, Piva JP, Garcia PC, Bered PL, Zilles K. Risk factors for extubation failure in mechanically ventilated pediatric patients. Pediatr Crit Care Med. 2005 Mar;6(2):16670 .

135 Kamm M, Burger R, Rimensberger P, Knoblauch A, Hammer J. Survey of children supported by long-term mechanical ventilation in Switzerland. Swiss Med Wkly. 2001 May; 131(19-20):261-6.

136 Cristea AI, Carroll AE, Davis SD, Swigonski 
NL, Ackerman VL. Outcomes of children with severe bronchopulmonary dysplasia who were ventilator dependent at home. Pediatrics. 2013 Sep;132(3):e727-34.

137 Gaies M, Tabbutt S, Schwartz SM, Bird GL, Alten JA, Shekerdemian LS, et al. Clinical Epidemiology of Extubation Failure in the Pediatric Cardiac ICU: A Report From the Pediatric Cardiac Critical Care Consortium. Pediatr Crit Care Med. 2015 Nov;16(9):83745.

138 Gupta P, McDonald R, Goyal S, Gossett JM, Imamura $\mathrm{M}$, Agarwal $\mathrm{A}$, et al. Extubation failure in infants with shunt-dependent pulmonary blood flow and univentricular physiology. Cardiol Young. 2014 Feb;24(1):6472.

139 Uhlig S, Frerichs I. [Lung protective ventilation - pathophysiology and diagnostics]. Anasthesiol Intensivmed Notfallmed Schmerzther. 2008 Jun;43(6):438-45.

140 Ibrahim EH, Ward S, Sherman G, Kollef $\mathrm{MH}$. A comparative analysis of patients with early-onset vs late-onset nosocomial pneumonia in the ICU setting. Chest. 2000 May; 117(5):1434-42.

141 Quinnell TG, Pilsworth S, Shneerson JM, Smith IE. Prolonged invasive ventilation following acute ventilatory failure in COPD: weaning results, survival, and the role of noninvasive ventilation. Chest. 2006 Jan; 129(1):133-9.

142 Damuth E, Mitchell JA, Bartock JL, Roberts BW, Trzeciak S. Long-term survival of critically ill patients treated with prolonged mechanical ventilation: a systematic review and meta-analysis. Lancet Respir Med. 2015 Jul; 3(7):544-53.

143 Cook D, Meade M, Guyatt G, Griffith L, Booker L. Criteria for weaning from mechanical ventilation. Evid Rep Technol Assess (Summ). 2000 Jun;23(23):1-4.

144 Ouellette DR, Patel S, Girard TD, Morris PE, Schmidt GA, Truwit JD, et al. Liberation From Mechanical Ventilation in Critically Ill Adults: An Official American College of Chest Physicians/American Thoracic Society Clinical Practice Guideline: Inspiratory Pressure Augmentation During Spontaneous Breathing Trials, Protocols Minimizing Sedation, and Noninvasive Ventilation Immediately After Extubation. Chest. 2017 Jan; 151(1):166-80.

145 Infarkt-bedingter kardiogener Schock - Diagnose, Monitoring und Therapie. Leitlinie der Deutschen Gesellschaft für Kardiologie - Herz- und Kreislaufforschung. Available from: www.awmf.org/leitlinien/aktuelle-leitlinien.

146 Baron R, Binder A, Biniek R, Braune S, Buerkle H, Dall P, et al.; DAS-Taskforce 2015. Evidence and consensus based guideline for the management of delirium, analgesia, and sedation in intensive care medicine. Revision 2015 (DAS-Guideline 2015) - short version. Ger Med Sci. 2015 Nov;13:Doc19.
147 Ely EW, Baker AM, Evans GW, Haponik EF. The prognostic significance of passing a daily screen of weaning parameters. Intensive Care Med. 1999 Jun;25(6):581-7.

148 Shehabi Y, Bellomo R, Reade MC, Bailey M, Bass F, Howe B, et al.; Sedation Practice in Intensive Care Evaluation (SPICE) Study Investigators; ANZICS Clinical Trials Group. Early intensive care sedation predicts longterm mortality in ventilated critically ill patients. Am J Respir Crit Care Med. 2012 Oct; 186(8):724-31.

149 Balzer F, Weiß B, Kumpf O, Treskatsch S, Spies C, Wernecke KD, et al. Early deep sedation is associated with decreased in-hospital and two-year follow-up survival. Crit Care. 2015 Apr;19(1):197.

150 Ramsay MA, Savege TM, Simpson BR, Goodwin R. Controlled sedation with alphaxalone-alphadolone. BMJ. 1974 Jun; 2(5920):656-9.

151 Riker RR, Picard JT, Fraser GL. Prospective evaluation of the Sedation-Agitation Scale for adult critically ill patients. Crit Care Med. 1999 Jul;27(7):1325-9.

152 Ely EW, Truman B, Shintani A, Thomason JW, Wheeler AP, Gordon S, et al. Monitoring sedation status over time in ICU patients: reliability and validity of the Richmond Agitation-Sedation Scale (RASS). JAMA. 2003 Jun;289(22):2983-91.

153 Sessler CN, Gosnell MS, Grap MJ, Brophy GM, O'Neal PV, Keane KA, et al. The Richmond Agitation-Sedation Scale: validity and reliability in adult intensive care unit patients. Am J Respir Crit Care Med. 2002 Nov; 166(10):1338-44.

154 Martin J, Franck M, Fischer M, Spies C. Sedation and analgesia in German intensive care units: how is it done in reality? Results of a patient-based survey of analgesia and sedation. Intensive Care Med. 2006 Aug;32(8): 1137-42.

155 Payen JF, Bru O, Bosson JL, Lagrasta A, Novel E, Deschaux I, et al. Assessing pain in critically ill sedated patients by using a behavioral pain scale. Crit Care Med. 2001 Dec; 29(12):2258-63.

156 Kress JP, Pohlman AS, O’Connor MF, Hall JB. Daily interruption of sedative infusions in critically ill patients undergoing mechanical ventilation. N Engl J Med. 2000 May; 342(20):1471-7.

157 Carson SS, Kress JP, Rodgers JE, Vinayak A, Campbell-Bright S, Levitt J, et al. A randomized trial of intermittent lorazepam versus propofol with daily interruption in mechanically ventilated patients. Crit Care Med. 2006 May;34(5):1326-32.

158 Girard TD, Kress JP, Fuchs BD, Thomason JW, Schweickert WD, Pun BT, et al. Efficacy and safety of a paired sedation and ventilator weaning protocol for mechanically ventilated patients in intensive care (Awakening and Breathing Controlled trial): a randomised controlled trial. Lancet. 2008 Jan; 371(9607):126-34.
159 Brook AD, Ahrens TS, Schaiff R, Prentice D, Sherman G, Shannon W, et al. Effect of a nursing-implemented sedation protocol on the duration of mechanical ventilation. Crit Care Med. 1999 Dec;27(12):2609-15.

160 Mehta S, Burry L, Cook D, Fergusson D, Steinberg M, Granton J, et al.; SLEAP Investigators; Canadian Critical Care Trials Group. Daily sedation interruption in mechanically ventilated critically ill patients cared for with a sedation protocol: a randomized controlled trial. JAMA. 2012 Nov; 308(19):1985-92.

161 Burry L, Rose L, McCullagh IJ. et al. Daily sedation interruption versus no daily sedation interruption for critically ill adult patients requiring invasive mechanical ventilation. Cochrane Database Syst Rev. 2014; 7:CD009176.

162 Invasive Beatmung und Einsatz extrakorporaler Verfahren bei akuter respiratorischer Insuffizienz. Leitlinie der Deutschen Gesellschaft für Anästhesiologie \& Intensivmedizin. Available from: www.awmf.org/leitlinien/aktuelle-leitlinien.

163 Ely EW, Gautam S, Margolin R, Francis J, May L, Speroff T, et al. The impact of delirium in the intensive care unit on hospital length of stay. Intensive Care Med. 2001 Dec;27(12):1892-900.

164 Ely EW, Margolin R, Francis J, May L, Truman B, Dittus R, et al. Evaluation of delirium in critically ill patients: validation of the Confusion Assessment Method for the Intensive Care Unit (CAM-ICU). Crit Care Med. 2001 Jul;29(7):1370-9.

165 Pandharipande P, Cotton BA, Shintani A, Thompson J, Costabile S, Truman Pun B, et al. Motoric subtypes of delirium in mechanically ventilated surgical and trauma intensive care unit patients. Intensive Care Med. 2007 Oct;33(10):1726-31.

166 Patel SB, Poston JT, Pohlman A, Hall JB, Kress JP. Rapidly reversible, sedation-related delirium versus persistent delirium in the intensive care unit. Am J Respir Crit Care Med. 2014 Mar;189(6):658-65.

167 Pandharipande PP, Girard TD, Jackson JC, Morandi A, Thompson JL, Pun BT, et al.; BRAIN-ICU Study Investigators. Longterm cognitive impairment after critical illness. N Engl J Med. 2013 Oct;369(14):130616.

168 Jackson JC, Pandharipande PP, Girard TD, Brummel NE, Thompson JL, Hughes CG, et al.; Bringing to light the Risk Factors And Incidence of Neuropsychological dysfunction in ICU survivors (BRAIN-ICU) study investigators. Depression, post-traumatic stress disorder, and functional disability in survivors of critical illness in the BRAINICU study: a longitudinal cohort study. Lancet Respir Med. 2014 May;2(5):369-79. 
169 Ely EW, Shintani A, Truman B, Speroff T, Gordon SM, Harrell FE Jr, et al. Delirium as a predictor of mortality in mechanically ventilated patients in the intensive care unit. JAMA. 2004 Apr;291(14):1753-62.

170 Devlin JW, Fong JJ, Schumaker G, O'Connor H, Ruthazer R, Garpestad E. Use of a validated delirium assessment tool improves the ability of physicians to identify delirium in medical intensive care unit patients. Crit Care Med. 2007 Dec;35(12):2721-4.

171 Hommelsheim C, Sichau M, Heipel R, Müller E, Gatermann S, Pfeifer M, et al. Predictors of Outcomes in Patients with Prolonged Weaning with Focus on Respiratory Tract Pathogens and Infection. Respiration. 2019; 97(2):135-44.

172 Bickenbach J, Schöneis D, Marx G, Marx N, Lemmen S, Dreher M. Impact of multidrugresistant bacteria on outcome in patients with prolonged weaning. BMC Pulm Med. 2018 Aug;18(1): 141.

173 Dalhoff K, Abele-Horn M, Andreas S, Deja M, Ewig S, Gastmeier P, et al.; Unter Mitwirkung der folgenden Wissenschaftlichen Fachgesellschaften und Institutionen: Deutsche Gesellschaft für Chirurgie; Deutsche Gesellschaft für Innere Medizin e.V.; Deutsche Gesellschaft für Internistische Intensivmedizin und Notfallmedizin; Deutsche Sepsis-Gesellschaft e.V.; und Robert Koch-Institut. [Epidemiology, Diagnosis and Treatment of Adult Patients with Nosocomial Pneumonia - Update 2017 - S3 Guideline of the German Society for Anaesthesiology and Intensive Care Medicine, the German Society for Infectious Diseases, the German Society for Hygiene and Microbiology, the German Respiratory Society and the Paul-Ehrlich-Society for Chemotherapy, the German Radiological Society and the Society for Virology]. Pneumologie. 2018 Jan; 72(1):15-63.

174 Esteban A, Alía I, Gordo F, Fernández R, Solsona JF, Vallverdú I, et al.; The Spanish Lung Failure Collaborative Group. Extubation outcome after spontaneous breathing trials with T-tube or pressure support ventilation. Am J Respir Crit Care Med. 1997 Aug;156(2 Pt 1):459-65.

175 Yang KL, Tobin MJ. A prospective study of indexes predicting the outcome of trials of weaning from mechanical ventilation. $\mathrm{N}$ Engl J Med. 1991 May;324(21):1445-50.

176 Perren A, Domenighetti G, Mauri S, Genini F, Vizzardi N. Protocol-directed weaning from mechanical ventilation: clinical outcome in patients randomized for a 30 -min or 120 -min trial with pressure support ventilation. Intensive Care Med. 2002 Aug;28(8): 1058-63.

177 Khamiees M, Raju P, DeGirolamo A, Amoateng-Adjepong Y, Manthous CA. Predictors of extubation outcome in patients who have successfully completed a spontaneous breathing trial. Chest. 2001 Oct; 120(4):1262-70.
178 Bach JR, Saporito LR. Criteria for extubation and tracheostomy tube removal for patients with ventilatory failure. A different approach to weaning. Chest. 1996 Dec;110(6): 1566-71.

179 Namen AM, Ely EW, Tatter SB, Case LD, Lucia MA, Smith A, et al. Predictors of successful extubation in neurosurgical patients. Am J Respir Crit Care Med. 2001 Mar;163(3 Pt 1):658-64.

180 Jubran A, Tobin MJ. Pathophysiologic basis of acute respiratory distress in patients who fail a trial of weaning from mechanical ventilation. Am J Respir Crit Care Med. 1997 Mar;155(3):906-15.

181 Laghi F, Cattapan SE, Jubran A, Parthasarathy S, Warshawsky P, Choi YS, et al. Is weaning failure caused by low-frequency fatigue of the diaphragm? Am J Respir Crit Care Med. 2003 Jan;167(2):120-7.

182 Matić I, Majerić-Kogler V. Comparison of pressure support and T-tube weaning from mechanical ventilation: randomized prospective study. Croat Med J. 2004 Apr;45(2): $162-6$.

183 Jones DP, Byrne P, Morgan C, Fraser I, Hyland R. Positive end-expiratory pressure vs T-piece. Extubation after mechanical ventilation. Chest. 1991 Dec;100(6):1655-9.

184 Haberthür C, Mols G, Elsasser S, Bingisser R, Stocker R, Guttmann J. Extubation after breathing trials with automatic tube compensation, T-tube, or pressure support ventilation. Acta Anaesthesiol Scand. 2002 Sep; 46(8):973-9.

185 Cohen J, Shapiro M, Grozovski E, Fox B, Lev $S$, Singer P. Prediction of extubation outcome: a randomised, controlled trial with automatic tube compensation vs. pressure support ventilation. Crit Care. 2009; 13(1):R21.

186 Kim WY, Suh HJ, Hong SB, Koh Y, Lim CM. Diaphragm dysfunction assessed by ultrasonography: influence on weaning from mechanical ventilation. Crit Care Med. 2011 Dec;39(12):2627-30.

187 DiNino E, Gartman EJ, Sethi JM, McCool FD. Diaphragm ultrasound as a predictor of successful extubation from mechanical ventilation. Thorax. 2014 May;69(5):423-7.

188 Soummer A, Perbet S, Brisson H, Arbelot C, Constantin JM, Lu Q, et al.; Lung Ultrasound Study Group. Ultrasound assessment of lung aeration loss during a successful weaning trial predicts postextubation distress*. Crit Care Med. 2012 Jul;40(7):2064-72.

189 Boulain T; Association des Réanimateurs du Centre-Ouest. Unplanned extubations in the adult intensive care unit: a prospective multicenter study. Am J Respir Crit Care Med. 1998 Apr;157(4 Pt 1):1131-7.

190 Blackwood B, Alderdice F, Burns K, Cardwell C, Lavery G, O'Halloran P. Use of weaning protocols for reducing duration of mechanical ventilation in critically ill adult patients: cochrane systematic review and meta-analysis. BMJ. 2011 Jan;342 jan13 2:c7237.
191 Blackwood B, Burns KE, Cardwell CR O'Halloran P. Protocolized versus non-protocolized weaning for reducing the duration of mechanical ventilation in critically ill adult patients. Cochrane Database Syst Rev. 2014 Nov;11(11):CD006904.

192 Frutos-Vivar F, Esteban A. When to wean from a ventilator: an evidence-based strategy. Cleve Clin J Med. 2003 May;70(5):389.

193 Girard TD, Ely EW. Protocol-driven ventilator weaning: reviewing the evidence. Clin Chest Med. 2008 Jun;29(2):241-52.

194 Vitacca M, Vianello A, Colombo D, Clini E, Porta R, Bianchi L, et al. Comparison of two methods for weaning patients with chronic obstructive pulmonary disease requiring mechanical ventilation for more than 15 days. Am J Respir Crit Care Med. 2001 Jul; 164(2):225-30.

195 Krishnan JA, Moore D, Robeson C, Rand CS, Fessler HE. A prospective, controlled trial of a protocol-based strategy to discontinue mechanical ventilation. Am J Respir Crit Care Med. 2004 Mar;169(6):673-8.

196 Dries DJ, McGonigal MD, Malian MS, Bor BJ, Sullivan C. Protocol-driven ventilator weaning reduces use of mechanical ventilation, rate of early reintubation, and ventilator-associated pneumonia. J Trauma. 2004 May;56(5):943-51.

197 Jaber S, Chanques G, Matecki S, Ramonatxo M, Vergne C, Souche B, et al. Post-extubation stridor in intensive care unit patients. Risk factors evaluation and importance of the cuff-leak test. Intensive Care Med. 2003 Jan;29(1):69-74.

198 De Bast Y, De Backer D, Moraine JJ, Lemaire $M$, Vandenborght C, Vincent JL. The cuff leak test to predict failure of tracheal extubation for laryngeal edema. Intensive Care Med. 2002 Sep;28(9):1267-72.

199 Fisher MM, Raper RF. The 'cuff-leak' test for extubation. Anaesthesia. 1992 Jan;47(1):10

200 Pluijms WA, van Mook WN, Wittekamp $\mathrm{BH}$, Bergmans DC. Postextubation laryngeal edema and stridor resulting in respiratory failure in critically ill adult patients: updated review. Crit Care. 2015 Sep;19(1):295.

201 Kuriyama A, Umakoshi N, Sun R. Prophylactic Corticosteroids for Prevention of Postextubation Stridor and Reintubation in Adults: A Systematic Review and Meta-analysis. Chest. 2017 May;151(5):1002-10.

202 Ochoa ME, Marín MC, Frutos-Vivar F, Gordo F, Latour-Pérez J, Calvo E, et al. Cuff-leak test for the diagnosis of upper airway obstruction in adults: a systematic review and meta-analysis. Intensive Care Med. 2009 Jul; 35(7):1171-9.

203 Lemyze M, Durville E, Meddour M, Jonard $\mathrm{M}$, Temime J, Barailler S, et al. Impact of fiber-optic laryngoscopy on the weaning process from mechanical ventilation in highrisk patients for postextubation stridor. Medicine(Baltimore).2017 Feb;96(5):e5971. 
204 Sutherasan Y, Theerawit P, Hongphanut T, Kiatboonsri C, Kiatboonsri S. Predicting laryngeal edema in intubated patients by portable intensive care unit ultrasound. J Crit Care. 2013 Oct;28(5):675-80.

205 Girard TD, Alhazzani W, Kress JP, Ouellette DR, Schmidt GA, Truwit JD, et al.; ATS/ CHEST Ad Hoc Committee on Liberation from Mechanical Ventilation in Adults. An Official American Thoracic Society/American College of Chest Physicians Clinical Practice Guideline: Liberation from Mechanical Ventilation in Critically Ill Adults. Rehabilitation Protocols, Ventilator Liberation Protocols, and Cuff Leak Tests. Am J Respir Crit Care Med. 2017 Jan;195(1):12033.

206 Schmidt GA, Girard TD, Kress JP, Morris PE, Ouellette DR, Alhazzani W, et al. Liberation from mechanical ventilation in critically ill adults: Executive Summary of an Official American College of Chest Physicians/ American Thoracic Society Clinical Practice Guideline. Chest. 2017 Jan;151(1):160-5.

207 Jaber S, Jung B, Chanques G, Bonnet F, Marret E. Effects of steroids on reintubation and post-extubation stridor in adults: metaanalysis of randomised controlled trials. Crit Care. 2009;13(2):R49.

208 Bein T, Bischoff M, Brückner U, Gebhardt $\mathrm{K}$, Henzler D, Hermes C, et al. S2e guideline: positioning and early mobilisation in prophylaxis or therapy of pulmonary disorders: Revision 2015: S2e guideline of the German Society of Anaesthesiology and Intensive Care Medicine (DGAI). Anaesthesist. 2015 Dec;64(Suppl 1):1-26.

209 Brower RG. Consequences of bed rest. Crit Care Med. 2009 Oct;37(10 Suppl):S422-8.

210 Hodgson CL, Bailey M, Bellomo R, Berney S, Buhr H, Denehy L, et al.; Trial of Early Activity and Mobilization Study Investigators. A Binational Multicenter Pilot Feasibility Randomized Controlled Trial of Early Goal-Directed Mobilization in the ICU. Crit Care Med. 2016 Jun;44(6): 1145-52.

211 Burtin C, Clerckx B, Robbeets C, Ferdinande $\mathrm{P}$, Langer D, Troosters T, et al. Early exercise in critically ill patients enhances short-term functional recovery. Crit Care Med. 2009 Sep;37(9):2499-505.

212 Morris PE, Goad A, Thompson C, Taylor K, Harry B, Passmore L, et al. Early intensive care unit mobility therapy in the treatment of acute respiratory failure. Crit Care Med. 2008 Aug;36(8):2238-43.

213 Schweickert WD, Pohlman MC, Pohlman AS, Nigos C, Pawlik AJ, Esbrook CL, et al. Early physical and occupational therapy in mechanically ventilated, critically ill patients: a randomised controlled trial. Lancet. 2009 May;373(9678):1874-82.
214 Schaller SJ, Anstey M, Blobner M, Edrich T, Grabitz SD, Gradwohl-Matis I, et al.; International Early SOMS-guided Mobilization Research Initiative. Early, goal-directed mobilisation in the surgical intensive care unit: a randomised controlled trial. Lancet. 2016 Oct;388(10052):1377-88.

215 Denehy L, Skinner EH, Edbrooke L, Haines K, Warrillow S, Hawthorne G, et al. Exercise rehabilitation for patients with critical illness: a randomized controlled trial with 12 months of follow-up. Crit Care. 2013 Jul; 17(4):R156.

216 Moss M, Nordon-Craft A, Malone D, Van Pelt D, Frankel SK, Warner ML, et al. A Randomized Trial of an Intensive Physical Therapy Program for Patients with Acute Respiratory Failure. Am J Respir Crit Care Med. 2016 May;193(10):1101-10.

217 Hodgson CL, Capell E, Tipping CJ. Early Mobilization of Patients in Intensive Care: Organization, Communication and Safety Factors that Influence Translation into Clinical Practice. Crit Care. 2018 Mar;22(1):77.

218 Morris PE, Berry MJ, Files DC, Thompson JC, Hauser J, Flores L, et al. Standardized Rehabilitation and Hospital Length of Stay Among Patients With Acute Respiratory Failure: A Randomized Clinical Trial. JAMA. 2016 Jun;315(24):2694-702.

219 Wright SE, Thomas K, Watson G, Baker C, Bryant A, Chadwick TJ, et al. Intensive versus standard physical rehabilitation therapy in the critically ill (EPICC): a multicentre, parallel-group, randomised controlled trial. Thorax. 2018 Mar;73(3):213-21.

220 Gerovasili V, Stefanidis K, Vitzilaios K, Karatzanos E, Politis P, Koroneos A, et al. Electrical muscle stimulation preserves the muscle mass of critically ill patients: a randomized study. Crit Care. 2009;13(5):R161.

221 Routsi C, Gerovasili V, Vasileiadis I, Karatzanos E, Pitsolis T, Tripodaki E, et al. Electrical muscle stimulation prevents critical illness polyneuromyopathy: a randomized parallel intervention trial. Crit Care. 2010; 14(2):R74.

222 Karatzanos E, Gerovasili V, Zervakis D, Tripodaki ES, Apostolou K, Vasileiadis I, et al. Electrical muscle stimulation: an effective form of exercise and early mobilization to preserve muscle strength in critically ill patients. Crit Care Res Pract. 2012;2012: 432752.

223 Weber-Carstens S, Schneider J, Wollersheim T, Assmann A, Bierbrauer J, Marg A, et al. Critical illness myopathy and GLUT4: significance of insulin and muscle contraction. Am J Respir Crit Care Med. 2013 Feb; 187(4):387-96.

224 Fischer A, Spiegl M, Altmann K, Winkler A, Salamon A, Themessl-Huber M, et al. Muscle mass, strength and functional outcomes in critically ill patients after cardiothoracic surgery: does neuromuscular electrical stimulation help? The Catastim 2 randomized controlled trial. Crit Care. 2016 Jan;20(1):30.
225 Nydahl P, Dewes M, Dubb R, Filipovic S, Hermes C, Jüttner F, et al.; Netzwerk Frühmobilisierung. [Early mobilization. Competencies, responsibilities, milestones]. Med Klin Intensivmed Notf Med. 2016 Mar; 111(2):153-9.

226 Jolley SE, Moss M, Needham DM, Caldwell E, Morris PE, Miller RR, et al.; Acute Respiratory Distress Syndrome Network Investigators. Point Prevalence Study of Mobilization Practices for Acute Respiratory Failure Patients in the United States. Crit Care Med. 2017 Feb;45(2):205-15.

227 Jolley SE, Caldwell E, Hough CL. Factors associated with receipt of physical therapy consultation in patients requiring prolonged mechanical ventilation. Dimens Crit Care Nurs. 2014 May-Jun;33(3):160-7.

228 Dubb R, Nydahl P, Hermes C, Schwabbauer N, Toonstra A, Parker AM, et al. Barriers and Strategies for Early Mobilization of Patients in Intensive Care Units. Ann Am Thorac Soc. 2016 May; 13(5):724-30.

229 Nickels M, Aitken LM, Walsham J, Watson L, McPhail S. Clinicians' perceptions of rationales for rehabilitative exercise in a critical care setting: A cross-sectional study. Aust Crit Care. 2017 Mar;30(2):79-84.

230 Nydahl P, Dubb R, Filipovic S, Hermes C, Jüttner F, Kaltwasser A, et al. Algorithmen zur Frühmobilisierung auf Intensivstationen. Med Klin Intensivmed Notf Med. 2017;112(2):156-62.

231 Schwabbauer N, Klarmann S, Geiseler J. Stellenwert der Atmungs- und Physiotherapie im Weaning. DIVI. 2017;2:70.

232 Jorch G, Kluge S, Markewitz A, et al. Empfehlungen zur Struktur und Ausstattung von Intensivstationen. DIVI. 2011;2:78-86.

233 Rollnik JD, Adolphsen J, Bauer J, et al. Prolongiertes Weaning in der neurologischneurochirurgischen Frührehabilitation. S2k-Leitlinie herausgegeben von der Weaning-Kommission der Deutschen Gesellschaft für Neurorehabilitation e.V. (DGNR). Der Nervenarzt. 2017;88:652-74.

234 Zanotti E, Felicetti G, Maini M, Fracchia C. Peripheral muscle strength training in bedbound patients with COPD receiving mechanical ventilation: effect of electrical stimulation. Chest. 2003 Jul;124(1):292-6.

235 Wageck B, Nunes GS, Silva FL, Damasceno MC, de Noronha M. Application and effects of neuromuscular electrical stimulation in critically ill patients: systematic review. Med Intensiva. 2014 Oct;38(7):444-54.

236 Bouletreau P, Patricot MC, Saudin F, Guiraud M, Mathian B. Effects of intermittent electrical stimulations on muscle catabolism in intensive care patients. JPEN J Parenter Enteral Nutr. 1987 Nov-Dec;11(6): 552-5.

237 Jones S, Man WD, Gao W, Higginson IJ, Wilcock A, Maddocks M. Neuromuscular electrical stimulation for muscle weakness in adults with advanced disease. Cochrane $\mathrm{Da}$ tabase Syst Rev. 2016 Oct;10:CD009419. 
238 Parry SM, Berney S, Warrillow S, El-Ansary D, Bryant AL, Hart N, et al. Functional electrical stimulation with cycling in the critically ill: a pilot case-matched control study. J Crit Care. 2014 Aug;29(4):695.e1-7.

239 Rollnik JD. Outcome of MRSA carriers in neurological early rehabilitation. BMC Neurol. 2014 Feb;14(1):34.

240 Gosselink R, Bott J, Johnson M, Dean E, Nava S, Norrenberg M, et al. Physiotherapy for adult patients with critical illness: recommendations of the European Respiratory Society and European Society of Intensive Care Medicine Task Force on Physiotherapy for Critically Ill Patients. Intensive Care Med. 2008 Jul;34(7):1188-99.

241 Sricharoenchai T, Parker AM, Zanni JM, Nelliot A, Dinglas VD, Needham DM. Safety of physical therapy interventions in critically ill patients: a single-center prospective evaluation of 1110 intensive care unit admissions. J Crit Care. 2014 Jun;29(3):395-400.

242 Bach JR, Bianchi C, Aufiero E. Oximetry and indications for tracheotomy for amyotrophic lateral sclerosis. Chest. 2004 Nov; 126(5):1502-7.

243 Smina M, Salam A, Khamiees M, Gada P, Amoateng-Adjepong Y, Manthous CA. Cough peak flows and extubation outcomes. Chest. 2003 Jul;124(1):262-8.

244 Beuret P, Roux C, Auclair A, Nourdine K, Kaaki M, Carton MJ. Interest of an objective evaluation of cough during weaning from mechanical ventilation. Intensive Care Med. 2009 Jun;35(6):1090-3.

245 Duan J, Zhou L, Xiao M, Liu J, Yang X. Semiquantitative cough strength score for predicting reintubation after planned extubation. Am J Crit Care. 2015 Nov;24(6):e86-90.

246 Smailes ST, McVicar AJ, Martin R. Cough strength, secretions and extubation outcome in burn patients who have passed a spontaneous breathing trial. Burns. 2013 Mar; 39(2):236-42.

247 Lai CC, Chen CM, Chiang SR, Liu WL, Weng SF, Sung MI, et al. Establishing predictors for successfully planned endotracheal extubation. Medicine (Baltimore). 2016 Oct;95(41):e4852.

248 Tobin MJ. Principles and Practice of Mechanical Ventilation. New York: McGrawHill; 1994.

249 Vassilakopoulos T, Zakynthinos S, Roussos C. Bench-to-bedside review: weaning failure-should we rest the respiratory muscles with controlled mechanical ventilation? Crit Care. 2006 Feb;10(1):204.

250 Vassilakopoulos T. Ventilator-induced diaphragm dysfunction: the clinical relevance of animal models. Intensive Care Med. 2008 Jan;34(1):7-16.

251 Sassoon CS, Caiozzo VJ, Manka A, Sieck GC. Altered diaphragm contractile properties with controlled mechanical ventilation. J Appl Physiol (1985). 2002 Jun;92(6):258595.

Prolonged Weaning: S2k Guideline by the German Respiratory Society
252 Levine S, Nguyen T, Taylor N, Friscia ME, Budak MT, Rothenberg P, et al. Rapid disuse atrophy of diaphragm fibers in mechanically ventilated humans. N Engl J Med. 2008 Mar; 358(13):1327-35.

253 Jaber S, Petrof BJ, Jung B, Chanques G, Berthet JP, Rabuel C, et al. Rapidly progressive diaphragmatic weakness and injury during mechanical ventilation in humans. Am J Respir Crit Care Med. 2011 Feb;183(3): 364-71.

254 Hermans G, Agten A, Testelmans D, Decramer M, Gayan-Ramirez G. Increased duration of mechanical ventilation is associated with decreased diaphragmatic force: a prospective observational study. Crit Care. 2010;14(4):R127.

255 Sassoon CS, Zhu E, Caiozzo VJ. Assist-control mechanical ventilation attenuates ventilator-induced diaphragmatic dysfunction. Am J Respir Crit Care Med. 2004 Sep;170(6): 626-32.

256 Prinianakis G, Delmastro M, Carlucci A, Ceriana $P$, Nava $S$. Effect of varying the pressurisation rate during noninvasive pressure support ventilation. Eur Respir J. 2004 Feb; 23(2):314-20.

257 Hoff FC, Tucci MR, Amato MB, Santos LJ, Victorino JA. Cycling-off modes during pressure support ventilation: effects on breathing pattern, patient effort, and comfort. J Crit Care. 2014 Jun;29(3):380-5.

258 Marini JJ, Rodriguez RM, Lamb V. The inspiratory workload of patient-initiated mechanical ventilation. Am Rev Respir Dis. 1986 Nov;134(5):902-9.

259 Harnisch LO, Zippel C, Herrmann P, Quintel M, Moerer O. Adjusting ventilator offcycling in invasively ventilated COPD patients needs comprehensive adjustments. Minerva Anestesiol. 2016 Jul;82(7):743-50.

260 Moerer O, Harnisch LO, Herrmann P, Zippel C, Quintel M. Patient-Ventilator Interaction During Noninvasive Ventilation in Simulated COPD. Respir Care. 2016 Jan; 61(1):15-22.

261 Brochard L, Harf A, Lorino H, Lemaire F. Inspiratory pressure support prevents diaphragmatic fatigue during weaning from mechanical ventilation. Am Rev Respir Dis. 1989 Feb;139(2):513-21.

262 Nava S, Bruschi C, Rubini F, Palo A, Iotti G, Braschi A. Respiratory response and inspiratory effort during pressure support ventilation in COPD patients. Intensive Care Med. 1995 Nov;21(11):871-9.

263 Imsand C, Feihl F, Perret C, Fitting JW. Regulation of inspiratory neuromuscular output during synchronized intermittent mechanical ventilation. Anesthesiology. 1994 Jan; 80(1):13-22.

264 Marini JJ, Smith TC, Lamb VJ. External work output and force generation during synchronized intermittent mechanical ventilation. Effect of machine assistance on breathing effort. Am Rev Respir Dis. 1988 Nov;138(5):1169-79.
265 Kirakli C, Naz I, Ediboglu O, Tatar D, Budak A, Tellioglu E. A randomized controlled trial comparing the ventilation duration between adaptive support ventilation and pressure assist/control ventilation in medical patients in the ICU. Chest. 2015 Jun; 147(6):1503-9.

266 Kirakli C, Ozdemir I, Ucar ZZ, Cimen P, Kepil S, Ozkan SA. Adaptive support ventilation for faster weaning in COPD: a randomised controlled trial. Eur Respir J. 2011 Oct;38(4):774-80.

267 Celli P, Privato E, Ianni S, Babetto C, D'Arena C, Guglielmo N, et al. Adaptive support ventilation versus synchronized intermittent mandatory ventilation with pressure support in weaning patients after orthotopic liver transplantation. Transplant Proc. 2014 Sep;46(7):2272-8.

268 Zhu F, Gomersall CD, Ng SK, Underwood MJ, Lee A. A randomized controlled trial of adaptive support ventilation mode to wean patients after fast-track cardiac valvular surgery. Anesthesiology. 2015 Apr;122(4):83240.

269 Sulzer CF, Chioléro R, Chassot PG, Mueller XM, Revelly JP. Adaptive support ventilation for fast tracheal extubation after cardiac surgery: a randomized controlled study. Anesthesiology. 2001 Dec;95(6):1339-45.

270 Gruber PC, Gomersall CD, Leung P, Joynt GM, Ng SK, Ho KM, et al. Randomized controlled trial comparing adaptive-support ventilation with pressure-regulated volumecontrolled ventilation with automode in weaning patients after cardiac surgery. Anesthesiology. 2008 Jul;109(1):81-7.

271 Dongelmans DA, Veelo DP, Paulus F, de Mol BA, Korevaar JC, Kudoga A, et al. Weaning automation with adaptive support ventilation: a randomized controlled trial in cardiothoracic surgery patients. Anesth Analg. 2009 Feb;108(2):565-71.

272 Petter AH, Chioléro RL, Cassina T, Chassot PG, Müller XM, Revelly JP. Automatic "respirator/weaning" with adaptive support ventilation: the effect on duration of endotracheal intubation and patient management. Anesth Analg. 2003 Dec;97(6):174350 .

273 Tassaux D, Dalmas E, Gratadour P, Jolliet P. Patient-ventilator interactions during partial ventilatory support: a preliminary study comparing the effects of adaptive support ventilation with synchronized intermittent mandatory ventilation plus inspiratory pressure support. Crit Care Med. 2002 Apr; 30(4):801-7.

274 Brochard L, Rauss A, Benito S, Conti G, Mancebo J, Rekik N, et al. Comparison of three methods of gradual withdrawal from ventilatory support during weaning from mechanical ventilation. Am J Respir Crit Care Med. 1994 Oct;150(4):896-903. 
275 Sinderby C, Beck J, Spahija J, Weinberg J, Grassino A. Voluntary activation of the human diaphragm in health and disease. J Appl Physiol (1985). 1998 Dec;85(6):2146-58.

276 Beck J, Weinberg J, Hamnegård CH, Spahija J, Olofson J, Grimby G, et al. Diaphragmatic function in advanced Duchenne muscular dystrophy. Neuromuscul Disord. 2006 Mar; 16(3):161-7.

277 Tuchscherer D, Z'graggen WJ, Passath C, Takala J, Sinderby C, Brander L. Neurally adjusted ventilatory assist in patients with critical illness-associated polyneuromyopathy. Intensive Care Med. 2011 Dec;37(12): 1951-61.

278 Rozé H, Repusseau B, Perrier V, Germain A, Séramondi R, Dewitte A, et al. Neuro-ventilatory efficiency during weaning from mechanical ventilation using neurally adjusted ventilatory assist. Br J Anaesth. 2013 Dec; 111(6):955-60.

279 Dres M, Schmidt M, Ferre A, Mayaux J, Similowski T, Demoule A. Diaphragm electromyographic activity as a predictor of weaning failure. Intensive Care Med. 2012 Dec;38(12):2017-25.

280 Barwing J, Pedroni C, Olgemöller U, Quintel $\mathrm{M}$, Moerer O. Electrical activity of the diaphragm (EAdi) as a monitoring parameter in difficult weaning from respirator: a pilot study. Crit Care. 2013 Aug;17(4):R182.

281 Trapp O, Fiedler M, Hartwich M, Schorl M, Kalenka A. Monitoring of Electrical Activity of the Diaphragm Shows Failure of T-Piece Trial Earlier than Protocol-Based Parameters in Prolonged Weaning in Non-communicative Neurological Patients. Neurocrit Care. 2017 Aug;27(1):35-43.

282 Muttini S, Villani PG, Trimarco R, Bellani G, Grasselli G, Patroniti N. Relation between peak and integral of the diaphragm electromyographic activity at different levels of support during weaning from mechanical ventilation: a physiologic study. J Crit Care. 2015 Feb;30(1):7-12.

283 Yonis H, Crognier L, Conil JM, Serres I, Rouget A, Virtos M, et al. Patient-ventilator synchrony in Neurally Adjusted Ventilatory Assist (NAVA) and Pressure Support Ventilation (PSV): a prospective observational study. BMC Anesthesiol. 2015 Aug;15(1): 117.

284 Delisle S, Ouellet P, Bellemare P, Tétrault JP, Arsenault P. Sleep quality in mechanically ventilated patients: comparison between NAVA and PSV modes. Ann Intensive Care. 2011 Sep;1(1):42.

285 Spahija J, de Marchie M, Albert M, Bellemare P, Delisle S, Beck J, et al. Patient-ventilator interaction during pressure support ventilation and neurally adjusted ventilatory assist. Crit Care Med. 2010 Feb;38(2):51826.
286 Thille AW, Rodriguez P, Cabello B, Lellouche F, Brochard L. Patient-ventilator asynchrony during assisted mechanical ventilation. Intensive Care Med. 2006 Oct; 32(10):1515-22.

287 Thille AW, Brochard L. Promoting PatientVentilator Synchrony. Clin Pulm Med. 2007;14(6):350-9.

288 de Wit M, Miller KB, Green DA, Ostman HE, Gennings C, Epstein SK. Ineffective triggering predicts increased duration of mechanical ventilation. Crit Care Med. 2009 Oct;37(10):2740-5.

289 Vagheggini G, Mazzoleni S, Vlad Panait E, Navalesi P, Ambrosino N. Physiologic response to various levels of pressure support and NAVA in prolonged weaning. Respir Med. 2013 Nov;107(11):1748-54.

290 Di Mussi R, Spadaro S, Mirabella L, Volta CA, Serio G, Staffieri F, et al. Impact of prolonged assisted ventilation on diaphragmatic efficiency: NAVA versus PSV. Crit Care. 2016 Jan;20(1):1.

291 Kuo NY, Tu ML, Hung TY, Liu SF, Chung $\mathrm{YH}$, Lin $\mathrm{MC}$, et al. A randomized clinical trial of neurally adjusted ventilatory assist versus conventional weaning mode in patients with COPD and prolonged mechanical ventilation. Int J Chron Obstruct Pulmon Dis. 2016 May;11:945-51.

292 Vitacca M, Clini E, Pagani M, Bianchi L, Rossi A, Ambrosino N. Physiologic effects of early administered mask proportional assist ventilation in patients with chronic obstructive pulmonary disease and acute respiratory failure. Crit Care Med. 2000 Jun;28(6):17917.

293 Grasso S, Puntillo F, Mascia L, Ancona G, Fiore T, Bruno F, et al. Compensation for increase in respiratory workload during mechanical ventilation. Pressure-support versus proportional-assist ventilation. Am J Respir Crit Care Med. 2000 Mar;161(3 Pt 1): 819-26.

294 Kondili E, Prinianakis G, Alexopoulou C, Vakouti E, Klimathianaki M, Georgopoulos D. Respiratory load compensation during mechanical ventilation-proportional assist ventilation with load-adjustable gain factors versus pressure support. Intensive Care Med. 2006 May;32(5):692-9.

295 Xirouchaki N, Kondili E, Vaporidi K, Xirouchakis G, Klimathianaki M, Gavriilidis G, et al. Proportional assist ventilation with load-adjustable gain factors in critically ill patients: comparison with pressure support. Intensive Care Med. 2008 Nov;34(11):202634.

296 Bosma K, Ferreyra G, Ambrogio C, Pasero D, Mirabella L, Braghiroli A, et al. Patientventilator interaction and sleep in mechanically ventilated patients: pressure support versus proportional assist ventilation. Crit Care Med. 2007 Apr;35(4):1048-54.
297 Bosma KJ, Read BA, Bahrgard Nikoo MJ Jones PM, Priestap FA, Lewis JF. A Pilot Randomized Trial Comparing Weaning From Mechanical Ventilation on Pressure Support Versus Proportional Assist Ventilation. Crit Care Med. 2016 Jun;44(6):1098108.

298 Räsänen J. IMPRV—synchronized APRV, or more? Intensive Care Med. 1992;18(2): 65-6.

299 De Jonghe B, Bastuji-Garin S, Durand MC, Malissin I, Rodrigues P, Cerf C, et al.; Groupe de Réflexion et d'Etude des Neuromyopathies en Réanimation. Respiratory weakness is associated with limb weakness and delayed weaning in critical illness. Crit Care Med. 2007 Sep;35(9):2007-15.

300 Carrie C, Gisbert-Mora C, Bonnardel E, Gauche B, Biais M, Vargas F, et al. Ultrasonographic diaphragmatic excursion is inaccurate and not better than the MRC score for predicting weaning-failure in mechanically ventilated patients. Anaesth Crit Care Pain Med. 2017 Feb;36(1):9-14.

301 Luo L, Li Y, Chen X, Sun B, Li W, Gu W, et al. Different effects of cardiac and diaphragm function assessed by ultrasound on extubation outcomes in difficult-to-wean patients: a cohort study. BMC Pulm Med. 2017 Dec;17(1):161.

302 Farghaly S, Hasan AA. Diaphragm ultrasound as a new method to predict extubation outcome in mechanically ventilated patients. Aust Crit Care. 2017 Jan;30(1):37-43.

303 Huang D, Ma H, Zhong W, Wang X, Wu Y, Qin T, et al. Using M-mode ultrasonography to assess diaphragm dysfunction and predict the success of mechanical ventilation weaning in elderly patients. J Thorac Dis. 2017 Sep;9(9):3177-86

304 Llamas-Álvarez AM, Tenza-Lozano EM, Latour-Pérez J. Diaphragm and Lung Ultrasound to Predict Weaning Outcome: Systematic Review and Meta-Analysis. Chest. 2017 Dec;152(6):1140-50.

305 Yoo JW, Lee SJ, Lee JD, Kim HC. Comparison of clinical utility between diaphragm excursion and thickening change using ultrasonography to predict extubation success. Korean J Intern Med (Korean Assoc Intern Med). 2018 Mar;33(2):331-9.

306 Ferrari G, De Filippi G, Elia F, Panero F, Volpicelli G, Aprà F. Diaphragm ultrasound as a new index of discontinuation from mechanical ventilation. Crit Ultrasound J. 2014 Jun;6(1):8.

307 Samanta S, Singh RK, Baronia AK, Poddar B, Azim A, Gurjar M. Diaphragm thickening fraction to predict weaning-a prospective exploratory study. J Intensive Care. 2017 Nov;5(1):62. 
308 Watson AC, Hughes PD, Louise Harris M, Hart N, Ware RJ, Wendon J, et al. Measurement of twitch transdiaphragmatic, esophageal, and endotracheal tube pressure with bilateral anterolateral magnetic phrenic nerve stimulation in patients in the intensive care unit. Crit Care Med. 2001 Jul;29(7): 1325-31.

309 Köhler D, Schönhofer B. Weaning nach Langzeitbeatmung bei Patienten mit erschöpfter Atempumpe - ein neues Behandlungskonzept. Med Klin. 1994;89:11-5.

310 Martin AD, Davenport PD, Franceschi AC, Harman E. Use of inspiratory muscle strength training to facilitate ventilator weaning: a series of 10 consecutive patients. Chest. 2002 Jul;122(1):192-6.

311 Martin AD, Smith BK, Davenport PD, Harman E, Gonzalez-Rothi RJ, Baz M, et al. Inspiratory muscle strength training improves weaning outcome in failure to wean patients: a randomized trial. Crit Care. 2011; 15(2):R84

312 Jubran A, Van de Graaff WB, Tobin MJ. Variability of patient-ventilator interaction with pressure support ventilation in patients with chronic obstructive pulmonary disease. Am J Respir Crit Care Med. 1995 Jul;152(1): 129-36.

313 Nava S, Bruschi C, Fracchia C, Braschi A, Rubini F. Patient-ventilator interaction and inspiratory effort during pressure support ventilation in patients with different pathologies. Eur Respir J. 1997 Jan;10(1):177-83.

314 Mancebo J, Amaro P, Mollo JL, Lorino H, Lemaire F, Brochard L. Comparison of the effects of pressure support ventilation delivered by three different ventilators during weaning from mechanical ventilation. Intensive Care Med. 1995 Nov;21(11):9139.

315 Dojat M, Harf A, Touchard D, Lemaire F, Brochard L. Clinical evaluation of a computer-controlled pressure support mode. Am J Respir Crit Care Med. 2000 Apr;161(4 Pt 1): 1161-6.

316 Lellouche F, Mancebo J, Jolliet P, Roeseler J, Schortgen F, Dojat M, et al. A multicenter randomized trial of computer-driven protocolized weaning from mechanical ventilation. Am J Respir Crit Care Med. 2006 Oct; 174(8):894-900.

317 Laghi F. Weaning: can the computer help? Intensive Care Med. 2008 Oct;34(10):17468.

318 Rose L, Presneill JJ, Johnston L, Cade JF. A randomised, controlled trial of conventional versus automated weaning from mechanical ventilation using SmartCare/PS. Intensive Care Med. 2008 Oct;34(10):1788-95.

319 Burns KE, Meade MO, Lessard MR, Hand L, Zhou Q, Keenan SP, et al. Wean earlier and automatically with new technology (the WEAN study). A multicenter, pilot randomized controlled trial. Am J Respir Crit Care Med. 2013 Jun;187(11):1203-11.

Prolonged Weaning: S2k Guideline by the German Respiratory Society
320 Draeger medical AG and CoKG. 2005 SmartCare. Knowledgebased system for clinical guidelines Sortware 1.1. Addendum to operating instructions Evita XL Software 6.0 or higher.

321 Bouadma L, Lellouche F, Cabello B, Taillé S, Mancebo J, Dojat M, et al. Computer-driven management of prolonged mechanical ventilation and weaning: a pilot study. Intensive Care Med. 2005 Oct;31(10):1446-50.

322 Rose L, Schultz MJ, Cardwell CR, Jouvet P, McAuley DF, Blackwood B. Automated versus non-automated weaning for reducing the duration of mechanical ventilation for critically ill adults and children. Cochrane Database Syst Rev. 2014 Jun;6(6):CD009235.

323 Taniguchi C, Victor ES, Pieri T, Henn R, Santana C, Giovanetti E, et al. Smart Care ${ }^{\mathrm{TM}}$ versus respiratory physiotherapy-driven manual weaning for critically ill adult patients: a randomized controlled trial. Crit Care. 2015 Jun;19(1):246.

324 Fry RW, Morton AR, Keast D. Periodisation of training stress-a review. Can J Sport Sci. 1992 Sep;17(3):234-40.

325 MacIntyre NR, Epstein SK, Carson S, Scheinhorn D, Christopher K, Muldoon S; National Association for Medical Direction of Respiratory Care. Management of patients requiring prolonged mechanical ventilation: report of a NAMDRC consensus conference. Chest. 2005 Dec;128(6):393754 .

326 Liang Z, Ren D, Choi J, Happ MB, Hravnak $M$, Hoffman LA. Music intervention during daily weaning trials-A 6 day prospective randomized crossover trial. Complement Ther Med. 2016 Dec;29:72-7.

327 Hetland B, Lindquist R, Weinert CR, PedenMcAlpine C, Savik K, Chlan L. Predictive Associations of Music, Anxiety, and Sedative Exposure on Mechanical Ventilation Weaning Trials. Am J Crit Care. 2017 May; 26(3):210-20.

328 Dellweg D, Barchfeld T, Haidl P, Appelhans P, Kohler D. Tracheostomy decannulation: implication on respiratory mechanics. Head Neck. 2007 Dec;29(12):1121-7.

329 Thille AW, Lyazidi A, Richard JC, Galia F, Brochard L. A bench study of intensivecare-unit ventilators: new versus old and turbine-based versus compressed gas-based ventilators. Intensive Care Med. 2009 Aug; 35(8):1368-76.

330 Richard JC, Carlucci A, Breton L, Langlais N, Jaber S, Maggiore S, et al. Bench testing of pressure support ventilation with three different generations of ventilators. Intensive Care Med. 2002 Aug;28(8):1049-57.

331 Brochard L, Thille AW. What is the proper approach to liberating the weak from mechanical ventilation? Crit Care Med. 2009 Oct;37(10 Suppl):S410-5.

332 Caroleo S, Agnello F, Abdallah K, Santangelo E, Amantea B. Weaning from mechanical ventilation: an open issue. Minerva Anestesiol. 2007 Jul-Aug;73(7-8):417-27.
333 Burns KE, Lellouche F, Loisel F, Slutsky AS, Meret A, Smith O, et al. Weaning critically ill adults from invasive mechanical ventilation: a national survey. Can J Anaesth. 2009 Aug;56(8):567-76.

334 Bapat P, Verghese C. Cuff deflation for easier weaning from ventilation. Br J Anaesth. 1997 Jul;79(1): 145

335 Shneerson JM. Are there new solutions to old problems with weaning? Br J Anaesth. 1997 Mar;78(3):238-40.

336 Fornataro-Clerici L, Zajac DJ. Aerodynamic characteristics of tracheostomy speaking valves. J Speech Hear Res. 1993 Jun;36(3): 529-32.

337 Johnson DC, Campbell SL, Rabkin JD. Tracheostomy tube manometry: evaluation of speaking valves, capping and need for downsizing. Clin Respir J. 2009 Jan;3(1):8-14.

338 Jiang TX, Reid WD, Road JD. Free radical scavengers and diaphragm injury following inspiratory resistive loading. Am J Respir Crit Care Med. 2001 Oct;164(7):1288-94.

339 Jubran A, Grant BJ, Duffner LA, Collins EG, Lanuza DM, Hoffman LA, et al. Effect of pressure support vs unassisted breathing through a tracheostomy collar on weaning duration in patients requiring prolonged mechanical ventilation: a randomized trial. JAMA. 2013 Feb;309(7):671-7.

340 Hubmayr RD, Abel MD, Rehder K. Physiologic approach to mechanical ventilation. Crit Care Med. 1990 Jan;18(1):103-13.

341 Petrof BJ, Legaré M, Goldberg P, Milic-Emili J, Gottfried SB. Continuous positive airway pressure reduces work of breathing and dyspnea during weaning from mechanical ventilation in severe chronic obstructive pulmonary disease. Am Rev Respir Dis. 1990 Feb;141(2):281-9.

342 Ranieri VM, Giuliani R, Cinnella G, Pesce C, Brienza N, Ippolito EL, et al. Physiologic effects of positive end-expiratory pressure in patients with chronic obstructive pulmonary disease during acute ventilatory failure and controlled mechanical ventilation. Am Rev Respir Dis. 1993 Jan;147(1):5-13.

343 Reissmann HK, Ranieri VM, Goldberg P, Gottfried SB. Continuous positive airway pressure facilitates spontaneous breathing in weaning chronic obstructive pulmonary disease patients by improving breathing pattern and gas exchange. Intensive Care Med. 2000 Dec;26(12):1764-72.

344 El-Khatib MF, Zeineldine SM, Jamaleddine GW. Effect of pressure support ventilation and positive end expiratory pressure on the rapid shallow breathing index in intensive care unit patients. Intensive Care Med. 2008 Mar;34(3):505-10.

345 Schmidt GB, Bombeck CT, Bennett EJ, Kotb $\mathrm{KM}$. Continuous positive airway pressure in the prophylaxis of the adult respiratory distress syndrome (ARDS). Langenbecks Arch Chir. 1975;Suppl:439-42. 
346 Ferreyra GP, Baussano I, Squadrone V, Richiardi L, Marchiaro G, Del Sorbo L, et al. Continuous positive airway pressure for treatment of respiratory complications after abdominal surgery: a systematic review and meta-analysis. Ann Surg. 2008 Apr;247(4): 617-26.

347 Nieszkowska A, Combes A, Luyt CE, Ksibi $\mathrm{H}$, Trouillet JL, Gibert C, et al. Impact of tracheotomy on sedative administration, sedation level, and comfort of mechanically ventilated intensive care unit patients. Crit Care Med. 2005 Nov;33(11):2527-33.

348 Pierson DJ. Tracheostomy and weaning. Respir Care. 2005 Apr;50(4):526-33.

349 Shapiro M, Wilson RK, Casar G, Bloom K, Teague RB. Work of breathing through different sized endotracheal tubes. Crit Care Med. 1986 Dec;14(12):1028-31.

350 Natalini G, Tuzzo DM, Comunale G, Rasulo FA, Amicucci G, Candiani A. Work of breathing-tidal volume relationship: analysis on an in vitro model and clinical implications. J Clin Monit Comput. 1999 Feb;15(2): 119-23.

351 Boqué MC, Gualis B, Sandiumenge A, Rello J. Endotracheal tube intraluminal diameter narrowing after mechanical ventilation: use of acoustic reflectometry. Intensive Care Med. 2004 Dec;30(12):2204-9.

352 Hewitt MS, Garland DE, Ayyoub Z. Heterotopic ossification complicating prolonged intubation: case report and review of the literature. J Spinal Cord Med. 2002;25(1):469.

353 Honig EG, Francis PB. Persistent tracheal dilatation: onset after brief mechanical ventilation with a "soft-cuff" endotracheal tube. South Med J. 1979 Apr;72(4):487-90.

354 Moons P, Sels K, De Becker W, De Geest S, Ferdinande P. Development of a risk assessment tool for deliberate self-extubation in intensive care patients. Intensive Care Med. 2004 Jul;30(7):1348-55.

355 Pandey CK, Singh N, Srivastava K, Alka R, Baronia A, Agarwal A, et al. Self-extubation in intensive care and re-intubation predictors: a retrospective study. J Indian Med Assoc. 2002 Jan;100(1):11.

356 Holzapfel L, Chevret S, Madinier G, Ohen F, Demingeon G, Coupry A, et al. Influence of long-term oro- or nasotracheal intubation on nosocomial maxillary sinusitis and pneumonia: results of a prospective, randomized, clinical trial. Crit Care Med. 1993 Aug;21(8): 1132-8.

357 Nordin U. The trachea and cuff-induced tracheal injury. An experimental study on causative factors and prevention. Acta Otolaryngol Suppl. 1977;345:1-71.

358 Ceriana P, Carlucci A, Navalesi P, Prinianakis G, Fanfulla F, Delmastro M, et al. Physiological responses during a $\mathrm{T}$-piece weaning trial with a deflated tube. Intensive Care Med. 2006 Sep;32(9):1399-403.
359 The Commission for Hospital Hygiene and Infection Prevention (KRINKO) at the Robert Koch Institute. [Prevention of nosocomial ventilator-associated pneumonia]. Bundesgesundheitsblatt Gesundheitsforschung Gesundheitsschutz. 2013 Nov;56(11): 1578-90.

360 Mao Z, Gao L, Wang G, Liu C, Zhao Y, Gu $\mathrm{W}$, et al. Subglottic secretion suction for preventing ventilator-associated pneumonia: an updated meta-analysis and trial sequential analysis. Crit Care. 2016 Oct;20(1):353.

361 Caroff DA, Li L, Muscedere J, Klompas M. Subglottic Secretion Drainage and Objective Outcomes: A Systematic Review and MetaAnalysis. Crit Care Med. 2016 Apr;44(4): 830-40.

362 Lin WC, Chen CW, Wang JD, Tsai LM. Is tracheostomy a better choice than translaryngeal intubation for critically ill patients requiring mechanical ventilation for more than 14 days? A comparison of short-term outcomes. BMC Anesthesiol. 2015 Dec; 15(1): 181

363 Mehta AB, Syeda SN, Bajpayee L, Cooke CR, Walkey AJ, Wiener RS. Trends in Tracheostomy for Mechanically Ventilated Patients in the United States, 1993-2012. Am J Respir Crit Care Med. 2015 Aug;192(4):446-54.

364 Kluge S, Baumann HJ, Nierhaus A, Kröger N, Meyer A, Kreymann G. Safety of percutaneous dilational tracheostomy in hematopoietic stem cell transplantation recipients requiring long-term mechanical ventilation. J Crit Care. 2008 Sep;23(3):394-8.

365 Vargas M, Sutherasan Y, Antonelli M, Brunetti I, Corcione A, Laffey JG, et al. Tracheostomy procedures in the intensive care unit: an international survey. Crit Care. 2015 Aug;19(1):291.

366 Wilson AM, Gray DM, Thomas JG. Increases in endotracheal tube resistance are unpredictable relative to duration of intubation. Chest. 2009 Oct;136(4):1006-13.

367 Durbin CG Jr. Tracheostomy: why, when, and how? Respir Care. 2010 Aug;55(8): 1056-68.

368 Maziak DE, Meade MO, Todd TR. The timing of tracheotomy: a systematic review. Chest. 1998 Aug;114(2):605-9.

369 Pilarczyk K, Haake N, Dudasova M, Huschens B, Wendt D, Demircioglu E, et al. Risk factors for bleeding complications after percutaneous dilatational tracheostomy: a ten-year institutional analysis. Anaesth Intensive Care. 2016 Mar;44(2):227-36.

370 Brass P, Hellmich M, Ladra A, Ladra J, Wrzosek A. Percutaneous techniques versus surgical techniques for tracheostomy. Cochrane Database Syst Rev. 2016 Jul; 7:CD008045.

371 Simon M, Metschke M, Braune SA, Püschel K, Kluge S. Death after percutaneous dilatational tracheostomy: a systematic review and analysis of risk factors. Crit Care. 2013 Oct; 17(5):R258.
372 Klemm E, Nowak AK. Tracheotomy-Related Deaths. Dtsch Arztebl Int. 2017 Apr; 114(16):273-9.

373 Dempsey GA, Morton B, Hammell C, Williams LT, Tudur Smith C, Jones T. LongTerm Outcome Following Tracheostomy in Critical Care: A Systematic Review. Crit Care Med. 2016 Mar;44(3):617-28.

374 Romero CM, Marambio A, Larrondo J, Walker K, Lira MT, Tobar E, et al. Swallowing dysfunction in nonneurologic critically ill patients who require percutaneous dilatational tracheostomy. Chest. 2010 Jun;137(6): 1278-82.

375 Bader CA, Keilmann A. [Swallowing Disorders in Tracheo(s)tomized Patients]. Laryngorhinootologie. 2017 May;96(5):280-92.

376 Rello J, Soñora R, Jubert P, Artigas A, Rué M, Vallés J. Pneumonia in intubated patients: role of respiratory airway care. Am J Respir Crit Care Med. 1996 Jul;154(1):111-5.

377 Heffner JE. Tracheal intubation in mechanically ventilated patients. Clin Chest Med. 1988 Mar;9(1):23-35.

378 Heuer B, Deller A. [Early and long-term results of percutaneous dilatation tracheostomy (PDT Ciaglia) in 195 intensive care patients]. Anasthesiol Intensivmed Notfallmed Schmerzther. 1998 May;33(5):306-12.

379 Muttini S, Melloni G, Gemma M, Casati A, Carretta A, Giudici D, et al. [Percutaneous or surgical trachetomy. Prospective, randomized comparison of the incidence of early and late complications]. Minerva Anestesiol. 1999 Jul-Aug;65(7-8):521-7.

380 Arabi YM, Alhashemi JA, Tamim HM, Esteban A, Haddad SH, Dawood A, et al. The impact of time to tracheostomy on mechanical ventilation duration, length of stay, and mortality in intensive care unit patients. J Crit Care. 2009 Sep;24(3):435-40.

381 Veenith T, Ganeshamoorthy S, Standley T, Carter J, Young P. Intensive care unit tracheostomy: a snapshot of UK practice. Int Arch Med. 2008 Oct;1(1):21.

382 Johnson-Obaseki S, Veljkovic A, Javidnia H. Complication rates of open surgical versus percutaneous tracheostomy in critically ill patients. Laryngoscope. 2016 Nov;126(11): 2459-67.

383 Putensen C, Theuerkauf N, Guenther U, Vargas M, Pelosi P. Percutaneous and surgical tracheostomy in critically ill adult patients: a meta-analysis. Crit Care. 2014 Dec; 18(6):544.

384 Dulguerov P, Gysin C, Perneger TV, Chevrolet JC. Percutaneous or surgical tracheostomy: a meta-analysis. Crit Care Med. 1999 Aug;27(8):1617-25.

385 Delaney A, Bagshaw SM, Nalos M. Percutaneous dilatational tracheostomy versus surgical tracheostomy in critically ill patients: a systematic review and meta-analysis. Crit Care. 2006;10(2):R55. 
386 Higgins KM, Punthakee X. Meta-analysis comparison of open versus percutaneous tracheostomy. Laryngoscope. 2007 Mar; 117(3):447-54.

387 Ciaglia P, Firsching R, Syniec C. Elective percutaneous dilatational tracheostomy. A new simple bedside procedure; preliminary report. Chest. 1985 Jun;87(6):715-9.

388 Schachner A, Ovil Y, Sidi J, Rogev M, Heilbronn Y, Levy MJ. Percutaneous tracheostomy - a new method. Crit Care Med. 1989 Oct;17(10):1052-6.

389 Griggs WM, Worthley LI, Gilligan JE, Thomas PD, Myburg JA. A simple percutaneous tracheostomy technique. Surg Gynecol Obstet. 1990 Jun;170(6):543-5.

390 Fantoni A, Ripamonti D. A non-derivative, non-surgical tracheostomy: the translaryngeal method. Intensive Care Med. 1997 Apr; 23(4):386-92.

391 Byhahn C, Wilke HJ, Halbig S, Lischke V, Westphal K. Percutaneous tracheostomy: ciaglia blue rhino versus the basic ciaglia technique of percutaneous dilational tracheostomy. Anesth Analg. 2000 Oct;91(4):8826.

392 Frova G, Quintel M. A new simple method for percutaneous tracheostomy: controlled rotating dilation. A preliminary report. Intensive Care Med. 2002 Mar;28(3):299-303.

393 Zgoda MA, Berger R. Balloon-facilitated percutaneous dilational tracheostomy tube placement: preliminary report of a novel technique. Chest. 2005 Nov;128(5):368890.

394 Sanabria A. Which percutaneous tracheostomy method is better? A systematic review. Respir Care. 2014 Nov;59(11):1660-70.

395 Marelli D, Paul A, Manolidis S, Walsh G, Odim JN, Burdon TA, et al. Endoscopic guided percutaneous tracheostomy: early results of a consecutive trial. J Trauma. 1990 Apr;30(4):433-5.

396 Fernandez L, Norwood S, Roettger R, Gass $\mathrm{D}$, Wilkins $\mathrm{H}$ 3rd. Bedside percutaneous tracheostomy with bronchoscopic guidance in critically ill patients. Arch Surg. 1996 Feb; 131(2):129-32.

397 Hinerman R, Alvarez F, Keller CA. Outcome of bedside percutaneous tracheostomy with bronchoscopic guidance. Intensive Care Med. 2000 Dec;26(12):1850-6.

398 Reilly PM, Sing RF, Giberson FA, Anderson HL 3rd, Rotondo MF, Tinkoff GH, et al. Hypercarbia during tracheostomy: a comparison of percutaneous endoscopic, percutaneous Doppler, and standard surgical tracheostomy. Intensive Care Med. 1997 Aug; 23(8):859-64.

399 Beiderlinden M, Karl Walz M, Sander A, Groeben H, Peters J. Complications of bronchoscopically guided percutaneous dilational tracheostomy: beyond the learning curve. Intensive Care Med. 2002 Jan;28(1):59-62.
400 Halum SL, Ting JY, Plowman EK, Belafsky PC, Harbarger CF, Postma GN, et al. A multi-institutional analysis of tracheotomy complications. Laryngoscope. 2012 Jan; 122(1):38-45.

401 Sustić A, Zupan Z, Eskinja N, Dirlić A, Bajek G. Ultrasonographically guided percutaneous dilatational tracheostomy after anterior cervical spine fixation. Acta Anaesthesiol Scand. 1999 Nov;43(10):1078-80.

402 Alansari M, Alotair H, Al Aseri Z, Elhoseny MA. Use of ultrasound guidance to improve the safety of percutaneous dilatational tracheostomy: a literature review. Crit Care. 2015 May;19(1):229.

403 Gobatto AL, Besen BA, Tierno PF, Mendes PV, Cadamuro F, Joelsons D, et al. Ultrasound-guided percutaneous dilational tracheostomy versus bronchoscopy-guided percutaneous dilational tracheostomy in critically ill patients (TRACHUS): a randomized noninferiority controlled trial. Intensive Care Med. 2016 Mar;42(3):342-51.

404 Sangwan YS, Chasse R. A modified technique for percutaneous dilatational tracheostomy: A retrospective review of 60 cases. J Crit Care. 2016 Feb;31(1):144-9.

405 Peris A, Linden M, Pellegrini G, Anichini V, Di Filippo A. Percutaneous dilatational tracheostomy: a self-drive control technique with video fiberoptic bronchoscopy reduces perioperative complications. Minerva Anestesiol. 2009 Jan-Feb;75(1-2):21-5.

406 Oberwalder M, Weis H, Nehoda H, KafkaRitsch R, Bonatti H, Prommegger R, et al. Videobronchoscopic guidance makes percutaneous dilational tracheostomy safer. Surg Endosc. 2004 May;18(5):839-42.

407 McGregor IA, Neill RS. Tracheostomy and the Björk flap. Lancet. 1983 Nov;2(8361): 1259.

408 Price DG. Techniques of tracheostomy for intensive care unit patients. Anaesthesia. 1983 Sep;38(9):902-4.

409 Bjork VO, Engstrom CG. The treatment of ventilatory insufficiency after pulmonary resection with tracheostomy and prolonged artificial ventilation. J Thorac Surg. 1955 Sep;30(3):356-67.

410 Heffner JE, Hess D. Tracheostomy management in the chronically ventilated patient. Clin Chest Med. 2001 Mar;22(1):55-69.

411 Cox CE, Carson SS, Holmes GM, Howard A, Carey TS. Increase in tracheostomy for prolonged mechanical ventilation in North Carolina, 1993-2002. Crit Care Med. 2004 Nov; 32(11):2219-26.

412 Heffner JE, Zamora CA. Clinical predictors of prolonged translaryngeal intubation in patients with the adult respiratory distress syndrome. Chest. 1990 Feb;97(2):447-52.

413 Sellers BJ, Davis BL, Larkin PW, Morris SE, Saffle JR. Early prediction of prolonged ventilator dependence in thermally injured patients. J Trauma. 1997 Dec;43(6):899-903.
414 Troché G, Moine P. Is the duration of mechanical ventilation predictable? Chest. 1997 Sep;112(3):745-51.

415 Möller MG, Slaikeu JD, Bonelli P, Davis AT Hoogeboom JE, Bonnell BW. Early tracheostomy versus late tracheostomy in the surgical intensive care unit. Am J Surg. 2005 Mar;189(3):293-6.

416 Rumbak MJ, Newton M, Truncale T, Schwartz SW, Adams JW, Hazard PB. A prospective, randomized, study comparing early percutaneous dilational tracheotomy to prolonged translaryngeal intubation (delayed tracheotomy) in critically ill medical patients. Crit Care Med. 2004 Aug;32(8): 1689-94.

417 Hsu CL, Chen KY, Chang CH, Jerng JS, Yu CJ, Yang PC. Timing of tracheostomy as a determinant of weaning success in critically ill patients: a retrospective study. Crit Care. 2005 Feb;9(1):R46-52.

418 Trouillet JL, Luyt CE, Guiguet M, Ouattara A, Vaissier E, Makri R, et al. Early percutaneous tracheotomy versus prolonged intubation of mechanically ventilated patients after cardiac surgery: a randomized trial. Ann Intern Med. 2011 Mar;154(6):373-83.

419 Young D, Harrison DA, Cuthbertson BH, Rowan K, TracMan Collaborators; TracMan Collaborators. Effect of early vs late tracheostomy placement on survival in patients receiving mechanical ventilation: the TracMan randomized trial. JAMA. 2013 May; 309(20):2121-9.

420 Terragni PP, Antonelli M, Fumagalli R, Faggiano $\mathrm{C}$, Berardino $\mathrm{M}$, Pallavicini $\mathrm{FB}$, et al. Early vs late tracheotomy for prevention of pneumonia in mechanically ventilated adult ICU patients: a randomized controlled trial. JAMA. 2010 Apr;303(15):1483-9.

421 McCredie VA, Alali AS, Scales DC, Adhikari NK, Rubenfeld GD, Cuthbertson BH, et al Effect of Early Versus Late Tracheostomy or Prolonged Intubation in Critically Ill $\mathrm{Pa}$ tients with Acute Brain Injury: A Systematic Review and Meta-Analysis. Neurocrit Care. 2017 Feb;26(1):14-25.

422 Meng L, Wang C, Li J, Zhang J. Early vs late tracheostomy in critically ill patients: a systematic review and meta-analysis. Clin Respir J. 2016 Nov;10(6):684-92.

423 Andriolo BN, Andriolo RB, Saconato H, Atallah ÁN, Valente O. Early versus late tracheostomy for critically ill patients. Cochrane Database Syst Rev. 2015 Jan; 1:CD007271.

424 Liu CC, Dort J. Re: Early versus late tracheostomy: a systematic review and meta-analysis. Otolaryngol Head Neck Surg. 2015 Apr; 152(4):768-9.

425 Cai SQ, Hu JW, Liu D, Bai XJ, Xie J, Chen JJ, et al. The influence of tracheostomy timing on outcomes in trauma patients: A metaanalysis. Injury. 2017 Apr;48(4):866-73. 
426 Szakmany T, Russell P, Wilkes AR, Hall JE. Effect of early tracheostomy on resource utilization and clinical outcomes in critically ill patients: meta-analysis of randomized controlled trials. Br J Anaesth. 2015 Mar;114(3): 396-405.

427 Huang H, Li Y, Ariani F, Chen X, Lin J. Timing of tracheostomy in critically ill patients: a meta-analysis. PLoS One. 2014 Mar; 9(3):e92981.

428 Shan L, Zhang R, Li LD. Effect of timing of tracheotomy on clinical outcomes: an update meta-analysis including 11 trials. Chin Med Sci J. 2013 Sep;28(3):159-66.

429 Siempos II, Ntaidou TK, Filippidis FT, Choi AM. Effect of early versus late or no tracheostomy on mortality and pneumonia of critically ill patients receiving mechanical ventilation: a systematic review and meta-analysis. Lancet Respir Med. 2015 Feb;3(2):150-8.

430 Hosokawa K, Nishimura M, Egi M, Vincent JL. Timing of tracheotomy in ICU patients: a systematic review of randomized controlled trials. Crit Care. 2015 Dec;19(1):424.

431 Ledgerwood LG, Salgado MD, Black H, Yoneda K, Sievers A, Belafsky PC. Tracheotomy tubes with suction above the cuff reduce the rate of ventilator-associated pneumonia in intensive care unit patients. Ann Otol Rhinol Laryngol. 2013 Jan;122(1):3-8.

432 Srinet P, Van Daele DJ, Adam SI, Burrell MI, Aronberg R, Leder SB. A Biomechanical Study of Hyoid Bone and Laryngeal Movements During Swallowing Comparing the Blom Low Profile Voice Inner Cannula and Passy-Muir One Way Tracheotomy Tube Speaking Valves. Dysphagia. 2015 Dec; 30(6):723-9.

433 Adam SI, Srinet P, Aronberg RM, Rosenberg G, Leder SB. Verbal communication with the Blom low profile and Passy-Muir oneway tracheotomy tube speaking valves. J Commun Disord. 2015 Jul-Aug;56:40-6.

434 Garguilo M, Leroux K, Lejaille M, Pascal S, Orlikowski D, Lofaso F, et al. Patient-controlled positive end-expiratory pressure with neuromuscular disease: effect on speech in patients with tracheostomy and mechanical ventilation support. Chest. 2013 May;143(5):1243-51.

435 Prigent $\mathrm{H}$, Orlikowski D, Blumen MB, Leroux $\mathrm{K}$, Legrand L, Lejaille $\mathrm{M}$, et al. Characteristics of tracheostomy phonation valves. Eur Respir J. 2006 May;27(5):992-6.

436 van den Boer C, Lansaat L, Muller SH, van den Brekel MW, Hilgers FJ. Comparative ex vivo study on humidifying function of three speaking valves with integrated heat and moisture exchanger for tracheotomised patients. Clin Otolaryngol. 2015 Dec;40(6): 616-21.

437 Marchese S, Corrado A, Scala R, Corrao S, Ambrosino N; Intensive Care Study Group, Italian Association of Hospital Pulmonologists (AIPO). Tracheostomy in patients with long-term mechanical ventilation: a survey. Respir Med. 2010 May;104(5):749-53.
438 Rosenblüh J, Schönhofer B, Kemper P, et al. Bedeutung von Platzhaltern tracheotomierter Patienten während der Entwöhnungsphasen nach Langzeitbeatmung. Med Klin (Munich). 1994;89:61-3.

439 Ferrer M, Esquinas A, Arancibia F, Bauer TT, Gonzalez G, Carrillo A, et al. Noninvasive ventilation during persistent weaning failure: a randomized controlled trial. Am J Respir Crit Care Med. 2003 Jul;168(1):70-6.

440 Girault C, Daudenthun I, Chevron V, Tamion F, Leroy J, Bonmarchand G. Noninvasive ventilation as a systematic extubation and weaning technique in acute-on-chronic respiratory failure: a prospective, randomized controlled study. Am J Respir Crit Care Med. 1999 Jul;160(1):86-92.

441 Ferrer M, Sellarés J, Valencia M, Carrillo A, Gonzalez G, Badia JR, et al. Non-invasive ventilation after extubation in hypercapnic patients with chronic respiratory disorders: randomised controlled trial. Lancet. 2009 Sep;374(9695):1082-8.

442 Bach JR, Goncalves M. Ventilator weaning by lung expansion and decannulation. Am J Phys Med Rehabil. 2004 Jul;83(7):560-8.

443 Bach JR, Gonçalves MR, Hamdani I, Winck JC. Extubation of patients with neuromuscular weakness: a new management paradigm. Chest. 2010 May;137(5):1033-9.

444 Lin MC, Liaw MY, Huang CC, Chuang ML, Tsai YH. Bilateral diaphragmatic paralysisa rare cause of acute respiratory failure managed with nasal mask bilevel positive airway pressure (BiPAP) ventilation. Eur Respir J. 1997 Aug;10(8):1922-4.

445 Pilcher DV, Bailey MJ, Treacher DF, Hamid S, Williams AJ, Davidson AC. Outcomes, cost and long term survival of patients referred to a regional weaning centre. Thorax. 2005 Mar;60(3):187-92.

446 Murphy PB, Rehal S, Arbane G, Bourke S, Calverley PM, Crook AM, et al. Effect of Home Noninvasive Ventilation With Oxygen Therapy vs Oxygen Therapy Alone on Hospital Readmission or Death After an Acute COPD Exacerbation: A Randomized Clinical Trial. JAMA. 2017 Jun;317(21): 2177-86.

447 Köhnlein T, Windisch W, Köhler D, Drabik A, Geiseler J, Hartl S, et al. Non-invasive positive pressure ventilation for the treatment of severe stable chronic obstructive pulmonary disease: a prospective, multicentre, randomised, controlled clinical trial. Lancet Respir Med. 2014 Sep;2(9):698-705.

448 Ward JJ. High-flow oxygen administration by nasal cannula for adult and perinatal patients. Respir Care. 2013 Jan;58(1):98-122.

449 Patel A, Nouraei SA. Transnasal Humidified Rapid-Insufflation Ventilatory Exchange (THRIVE): a physiological method of increasing apnoea time in patients with difficult airways. Anaesthesia. 2015 Mar;70(3): 323-9.
450 Goligher EC, Slutsky AS. Not Just Oxygen? Mechanisms of Benefit from High-Flow Nasal Cannula in Hypoxemic Respiratory Failure. Am J Respir Crit Care Med. 2017 May; 195(9):1128-31

451 Christopher KL, Schwartz MD. Transtracheal oxygen therapy. Chest. 2011 Feb; 139(2):435-40.

452 Schönhofer B, Wenzel M, Wiemann J, et al. Transtracheale Sauerstoffinsufflation: Reduktion der Atemarbeit in der Entwöhnung vom Respirator nach Langzeitbeatmung. Intensivmed. 1995;32:199-204.

453 Schönhofer B, Geibel M, Stickeler P, Wenzel M, Köhler D. Endoscopic placement of a tracheal oxygen catheter: a new technique. Intensive Care Med. 1997 Apr;23(4):445-9.

454 Nuckton TJ, Alonso JA, Kallet RH, Daniel BM, Pittet JF, Eisner MD, et al. Pulmonary dead-space fraction as a risk factor for death in the acute respiratory distress syndrome. $\mathrm{N}$ Engl J Med. 2002 Apr;346(17):1281-6.

455 Frat JP, Thille AW, Mercat A, Girault C, Ragot S, Perbet S, et al.; FLORALI Study Group; REVA Network. High-flow oxygen through nasal cannula in acute hypoxemic respiratory failure. N Engl J Med. 2015 Jun; 372(23):2185-96.

456 Stéphan F, Barrucand B, Petit P, RézaiguiaDelclaux S, Médard A, Delannoy B, et al.; BiPOP Study Group. High-Flow Nasal Oxygen vs Noninvasive Positive Airway Pressure in Hypoxemic Patients After Cardiothoracic Surgery: A Randomized Clinical Trial. JAMA. 2015 Jun;313(23):2331-9.

457 Hernández G, Vaquero C, González P, Subira C, Frutos-Vivar F, Rialp G, et al. Effect of Postextubation High-Flow Nasal Cannula vs Conventional Oxygen Therapy on Reintubation in Low-Risk Patients: A Randomized Clinical Trial. JAMA. 2016 Apr;315(13): 1354-61.

458 Hernández G, Vaquero C, Colinas L, Cuena R, González P, Canabal A, et al. Effect of Postextubation High-Flow Nasal Cannula vs Noninvasive Ventilation on Reintubation and Postextubation Respiratory Failure in High-Risk Patients: A Randomized Clinical Trial. JAMA. 2016 Oct;316(15):1565-74.

459 Doshi P, Whittle JS, Bublewicz M, Kearney J, Ashe T, Graham R, et al. High-Velocity Nasal Insufflation in the Treatment of Respiratory Failure: A Randomized Clinical Trial. Ann Emerg Med. 2018 Jul;72(1):73-83.e5.

460 Monro-Somerville T, Sim M, Ruddy J, Vilas M, Gillies MA. The Effect of High-Flow Nasal Cannula Oxygen Therapy on Mortality and Intubation Rate in Acute Respiratory Failure: A Systematic Review and MetaAnalysis. Crit Care Med. 2017 Apr; 45(4):e449-56.

461 Zhu Y, Yin H, Zhang R, Wei J. High-flow nasal cannula oxygen therapy versus conventional oxygen therapy in patients with acute respiratory failure: a systematic review and meta-analysis of randomized controlled trials. BMC Pulm Med. 2017 Dec;17(1):201. 
462 Corley A, Rickard CM, Aitken LM, Johnston A, Barnett A, Fraser JF, et al. High-flow nasal cannulae for respiratory support in adult intensive care patients. Cochrane Database Syst Rev. 2017 May;5:CD010172.

463 Ni YN, Luo J, Yu H, Liu D, Ni Z, Cheng J, et al. Can High-flow Nasal Cannula Reduce the Rate of Endotracheal Intubation in Adult Patients With Acute Respiratory Failure Compared With Conventional Oxygen Therapy and Noninvasive Positive Pressure Ventilation?: A Systematic Review and Meta-analysis. Chest. 2017 Apr;151(4):76475.

464 Ischaki E, Pantazopoulos I, Zakynthinos S. Nasal high flow therapy: a novel treatment rather than a more expensive oxygen device. Eur Respir Rev. 2017 Aug;26(145):145.

465 Ou X, Hua Y, Liu J, Gong C, Zhao W. Effect of high-flow nasal cannula oxygen therapy in adults with acute hypoxemic respiratory failure: a meta-analysis of randomized controlled trials. CMAJ. 2017 Feb;189(7):E260-7.

466 Leeies M, Flynn E, Turgeon AF, Paunovic B, Loewen H, Rabbani R, et al. High-flow oxygen via nasal cannulae in patients with acute hypoxemic respiratory failure: a systematic review and meta-analysis. Syst Rev. 2017 Oct;6(1):202.

467 Zhao H, Wang H, Sun F, Lyu S, An Y. Highflow nasal cannula oxygen therapy is superior to conventional oxygen therapy but not to noninvasive mechanical ventilation on intubation rate: a systematic review and meta-analysis. Crit Care. 2017 Jul;21(1):184.

468 Nedel WL, Deutschendorf C, Moraes Rodrigues Filho E. High-Flow Nasal Cannula in Critically Ill Subjects With or at Risk for Respiratory Failure: A Systematic Review and Meta-Analysis. Respir Care. 2017 Jan; 62(1):123-32.

469 Liatsi D, Tsapas B, Pampori S, Tsagourias M, Pneumatikos I, Matamis D. Respiratory, metabolic and hemodynamic effects of clonidine in ventilated patients presenting with withdrawal syndrome. Intensive Care Med. 2009 Feb;35(2):275-81.

470 Natalini G, Di Maio A, Rosano A, Ferretti P, Bertelli M, Bernardini A. Remifentanil improves breathing pattern and reduces inspiratory workload in tachypneic patients. Respir Care. 2011 Jun;56(6):827-33.

471 Heunks LM, van der Hoeven JG. Clinical review: the $\mathrm{ABC}$ of weaning failure-a structured approach. Crit Care. 2010;14(6):245.

472 Bancalari E, Claure N. Strategies to accelerate weaning from respiratory support. Early Hum Dev. 2013 Jun;89(Suppl 1):S4-6.

473 Parnell H, Quirke G, Farmer S, Adeyemo S, Varney V. The successful treatment of hypercapnic respiratory failure with oral modafinil. Int $J$ Chron Obstruct Pulmon Dis. 2014 Apr;9:413-9.
474 Kim DW, Joo JD, In JH, Jeon YS, Jung HS, Jeon $\mathrm{KB}$, et al. Comparison of the recovery and respiratory effects of aminophylline and doxapram following total intravenous anesthesia with propofol and remifentanil. J Clin Anesth. 2013 May;25(3):173-6.

475 Abbasi S, Farsaei S, Fazel K, Golzari SE, Mahmoodpoor A. Can donepezil facilitate weaning from mechanical ventilation in difficult to wean patients? An interventional pilot study. Daru. 2015 Mar;23(1):23.

476 Williams SC, Marshall NS, Kennerson M, Rogers NL, Liu PY, Grunstein RR. Modafinil effects during acute continuous positive airway pressure withdrawal: a randomized crossover double-blind placebo-controlled trial. Am J Respir Crit Care Med. 2010 Apr; 181(8):825-31.

477 Faisy C, Meziani F, Planquette B, Clavel M, Gacouin A, Bornstain C, et al.; DIABOLO Investigators. Effect of Acetazolamide vs Placebo on Duration of Invasive Mechanical Ventilation Among Patients With Chronic Obstructive Pulmonary Disease: A Randomized Clinical Trial. JAMA. 2016 Feb; 315(5):480-8.

478 Rialp G, Raurich JM, Llompart-Pou JA, Ayestarán I, Ibáñez J. Respiratory CO2 response depends on plasma bicarbonate concentration in mechanically ventilated patients. Med Intensiva. 2014 May;38(4):203-10.

479 Beattie WS, Karkouti K, Wijeysundera DN, Tait G. Risk associated with preoperative anemia in noncardiac surgery: a single-center cohort study. Anesthesiology. 2009 Mar; 110(3):574-81.

480 Carrascal Y, Maroto L, Rey J, Arévalo A, Arroyo J, Echevarría JR, et al. Impact of preoperative anemia on cardiac surgery in octogenarians. Interact Cardiovasc Thorac Surg. 2010 Feb;10(2):249-55.

481 De Santo L, Romano G, Della Corte A, de Simone V, Grimaldi F, Cotrufo M, et al. Preoperative anemia in patients undergoing coronary artery bypass grafting predicts acute kidney injury. J Thorac Cardiovasc Surg. 2009 Oct;138(4):965-70.

482 Karkouti K, Wijeysundera DN, Beattie WS; Reducing Bleeding in Cardiac Surgery (RBC) Investigators. Risk associated with preoperative anemia in cardiac surgery: a multicenter cohort study. Circulation. 2008 Jan;117(4):478-84.

$483 \mathrm{Wu}$ WC, Schifftner TL, Henderson WG, Eaton CB, Poses RM, Uttley G, et al. Preoperative hematocrit levels and postoperative outcomes in older patients undergoing noncardiac surgery. JAMA. 2007 Jun;297(22):2481-8.

484 Kulier A, Levin J, Moser R, RumpoldSeitlinger G, Tudor IC, Snyder-Ramos SA, et al.; Investigators of the Multicenter Study of Perioperative Ischemia Research Group; Ischemia Research and Education Foundation. Impact of preoperative anemia on outcome in patients undergoing coronary artery bypass graft surgery. Circulation. 2007 Jul;116(5):471-9.
485 Wu WC, Smith TS, Henderson WG, Eaton $\mathrm{CB}$, Poses RM, Uttley G, et al. Operative blood loss, blood transfusion, and 30-day mortality in older patients after major noncardiac surgery. Ann Surg. 2010 Jul;252(1): 11-7.

486 Klein HG, Spahn DR, Carson JL. Red blood cell transfusion in clinical practice. Lancet. 2007 Aug;370(9585):415-26.

487 Wang JK, Klein HG. Red blood cell transfusion in the treatment and management of anaemia: the search for the elusive transfusion trigger. Vox Sang. 2010 Jan;98(1):2-11.

488 Viires N, Sillye G, Aubier M, Rassidakis A, Roussos C. Regional blood flow distribution in dog during induced hypotension and low cardiac output. Spontaneous breathing versus artificial ventilation. J Clin Invest. 1983 Sep;72(3):935-47.

489 Holst LB, Haase N, Wetterslev J, Wernerman J, Guttormsen AB, Karlsson S, et al. TRISS Trial Group; Scandinavian Critical Care Trials Group. Lower versus higher hemoglobin threshold for transfusion in septic shock. N Engl J Med. 2014 Oct;371(15): 1381-91.

490 Murphy GJ, Pike K, Rogers CA, Wordsworth S, Stokes EA, Angelini GD, et al.; TITRe2 Investigators. Liberal or restrictive transfusion after cardiac surgery. N Engl J Med. 2015 Mar;372(11):997-1008.

491 Mazer CD, Whitlock RP, Fergusson DA, Hall J, Belley-Cote E, Connolly K, et al.; TRICS Investigators and Perioperative Anesthesia Clinical Trials Group. Restrictive or Liberal Red-Cell Transfusion for Cardiac Surgery. N Engl J Med. 2017 Nov;377(22): 2133-44.

492 Odutayo A, Desborough MJ, Trivella M Stanley AJ, Dorée C, Collins GS, et al. Restrictive versus liberal blood transfusion for gastrointestinal bleeding: a systematic review and meta-analysis of randomised controlled trials. Lancet Gastroenterol Hepatol. 2017 May;2(5):354-60.

493 Cornet AD, ZwartE, KingmaSD, Groeneveld AB. Pulmonary effects of red blood cell transfusion in critically ill, non-bleeding patients. Transfus Med. 2010 Aug;20(4):2216.

494 Zilberberg MD, Shorr AF. Effect of a restrictive transfusion strategy on transfusion-attributable severe acute complications and costs in the US ICUs: a model simulation. BMC Health Serv Res. 2007 Aug;7(1):138.

495 Zilberberg MD, Stern LS, Wiederkehr DP, Doyle JJ, Shorr AF. Anemia, transfusions and hospital outcomes among critically ill patients on prolonged acute mechanical ventilation: a retrospective cohort study. Crit Care. 2008;12(2):R60.

496 Hendrickson JE, Hillyer CD. Noninfectious serious hazards of transfusion. Anesth Analg. 2009 Mar;108(3):759-69. 
497 Rohde JM, Dimcheff DE, Blumberg N, Saint S, Langa KM, Kuhn L, et al. Health care-associated infection after red blood cell transfusion: a systematic review and meta-analysis. JAMA. 2014 Apr;311(13):1317-26.

498 Carson JL, Stanworth SJ, Roubinian N, Fergusson DA, Triulzi D, Doree C, et al. Transfusion thresholds and other strategies for guiding allogeneic red blood cell transfusion. Cochrane Database Syst Rev. 2016 Oct; 10:CD002042.

499 American Society of Anesthesiologists Task Force on Perioperative Blood Management. Practice guidelines for perioperative blood management: an updated report by the American Society of Anesthesiologists Task Force on Perioperative Blood Management*. Anesthesiology. 2015 Feb;122(2): 241-75.

500 Carson JL, Grossman BJ, Kleinman S, Tinmouth AT, Marques MB, Fung MK, et al.; Clinical Transfusion Medicine Committee of the AABB. Red blood cell transfusion: a clinical practice guideline from the AABB* Ann Intern Med. 2012 Jul;157(1):49-58.

501 Bundesärztekammer. Querschnitts-Leitlinien (BÄK) zur Therapie mit Blutkomponenten und Plasmaderivaten - 4. aktualisierte und. überarbeitete Auflage. Köln: Deutscher Ärzte-Verlag; 2015.

502 Dellinger RP, Levy MM, Rhodes A, Annane D, Gerlach H, Opal SM, et al.; Surviving Sepsis Campaign Guidelines Committee including The Pediatric Subgroup. Surviving Sepsis Campaign: international guidelines for management of severe sepsis and septic shock, 2012. Intensive Care Med. 2013 Feb; 39(2):165-228.

503 Qaseem A, Humphrey LL, Fitterman N, Starkey M, Shekelle P; Clinical Guidelines Committee of the American College of Physicians. Treatment of anemia in patients with heart disease: a clinical practice guideline from the American College of Physicians. Ann Intern Med. 2013 Dec;159(11): 770-9.

504 Schönhofer B, Böhrer H, Köhler D. Blood transfusion facilitating difficult weaning from the ventilator. Anaesthesia. $1998 \mathrm{Feb}$; 53(2):181-4

505 Orlov D, O’Farrell R, McCluskey SA, Carroll J, Poonawala H, Hozhabri S, et al. The clinical utility of an index of global oxygenation for guiding red blood cell transfusion in cardiac surgery. Transfusion. 2009 Apr;49(4): 682-8.

506 Vallet B, Adamczyk S, Barreau O, Lebuffe G. Physiologic transfusion triggers. Best Pract Res Clin Anaesthesiol. 2007 Jun;21(2):17381.

507 Adamczyk S, Robin E, Barreau O, Fleyfel M, Tavernier B, Lebuffe G, et al. [Contribution of central venous oxygen saturation in postoperative blood transfusion decision]. Ann Fr Anesth Reanim. 2009 Jun;28(6):522-30.
508 Vallet B, Robin E, Lebuffe G. Venous oxygen saturation as a physiologic transfusion trigger. Crit Care. 2010;14(2):213.

509 Vuille-Lessard E, Boudreault D, Girard F, Ruel M, Chagnon M, Hardy JF. Red blood cell transfusion practice in elective orthopedic surgery: a multicenter cohort study. Transfusion. 2010 Oct;50(10):2117-24.

510 Hébert PC, Tinmouth A, Corwin HL. Controversies in RBC transfusion in the critically ill. Chest. 2007 May;131(5):1583-90.

511 Zeroual N, Samarani G, Gallais J, Culas G, Saour M, Mourad M, et al. ScvO2 changes after red-blood-cell transfusion for anaemia in cardiothoracic and vascular ICU patients: an observational study. Vox Sang. 2018 Feb; 113(2):136-42.

512 Römers LH, Bakker C, Dollée N, Hoeks SE, Lima A, Raat NJ, et al. Cutaneous Mitochondrial PO2, but Not Tissue Oxygen Saturation, Is an Early Indicator of the Physiologic Limit of Hemodilution in the Pig. Anesthesiology. 2016 Jul;125(1):124-32.

513 Cahill NE, Dhaliwal R, Day AG, Jiang X, Heyland DK. Nutrition therapy in the critical care setting: what is "best achievable" practice? An international multicenter observational study. Crit Care Med. 2010 Feb; 38(2):395-401.

514 Compher C, Chittams J, Sammarco T, Nicolo M, Heyland DK. Greater Protein and Energy Intake May Be Associated With Improved Mortality in Higher Risk Critically Ill Patients: A Multicenter, Multinational Observational Study. Crit Care Med. 2017 Feb; 45(2):156-63

515 Hill GL. Impact of nutritional support on the clinical outcome of the surgical patient. Clin Nutr. 1994 Dec;13(6):331-40.

516 Plank LD, Hill GL. Energy balance in critical illness. Proc Nutr Soc. 2003 May;62(2):54552 .

517 Herve P, Simonneau G, Girard P, Cerrina J, Mathieu M, Duroux P. Hypercapnic acidosis induced by nutrition in mechanically ventilated patients: glucose versus fat. Crit Care Med. 1985 Jul;13(7):537-40.

518 McClave SA, Taylor BE, Martindale RG, Warren MM, Johnson DR, Braunschweig C, et al.; Society of Critical Care Medicine; American Society for Parenteral and Enteral Nutrition. Guidelines for the Provision and Assessment of Nutrition Support Therapy in the Adult Critically Ill Patient: Society of Critical Care Medicine (SCCM) and American Society for Parenteral and Enteral Nutrition (A.S.P.E.N.). JPEN J Parenter Enteral Nutr. 2016 Feb;40(2):159-211.

519 Reintam Blaser A, Starkopf J, Alhazzani W, Berger MM, Casaer MP, Deane AM, et al.; ESICM Working Group on Gastrointestinal Function. Early enteral nutrition in critically ill patients: ESICM clinical practice guidelines. Intensive Care Med. 2017 Mar;43(3):380-98.
520 al-Saady NM, Blackmore CM, Bennett ED. High fat, low carbohydrate, enteral feeding lowers $\mathrm{PaCO} 2$ and reduces the period of ventilation in artificially ventilated patients. Intensive Care Med. 1989;15(5):290-5

521 van den Berg B, Bogaard JM, Hop WC. High fat, low carbohydrate, enteral feeding in patients weaning from the ventilator. Intensive Care Med. 1994 Aug;20(7):470-5.

522 Mesejo A, Acosta JA, Ortega C, Vila J, Fernández M, Ferreres J, et al. Comparison of a high-protein disease-specific enteral formula with a high-protein enteral formula in hyperglycemic critically ill patients. Clin Nutr. 2003 Jun;22(3):295-305.

523 Kostadima E, Kaditis AG, Alexopoulos EI, Zakynthinos E, Sfyras D. Early gastrostomy reduces the rate of ventilator-associated pneumonia in stroke or head injury patients. Eur Respir J. 2005 Jul;26(1):106-11.

524 Elke G, Hartl WH, Kreymann KG, et al DGEM-Leitlinie: „Klinische Ernährung in der Intensivmedizin“. 2018 Available from: www.awmf.org/leitlinien/aktuelle-leitlinien Register-Nummer 073-004.

525 Peterson SJ, Tsai AA, Scala CM, Sowa DC, Sheean PM, Braunschweig CL. Adequacy of oral intake in critically ill patients 1 week after extubation. J Am Diet Assoc. 2010 Mar 110(3):427-33.

526 Kreymann G, Adolph M, Mueller MJ Working group for developing the guidelines for parenteral nutrition of The German Association for Nutritional Medicine. Energy expenditure and energy intake - Guidelines on Parenteral Nutrition, Chapter 3. Ger Med Sci. 2009 Nov; 7:Doc25.

527 Zusman O, Theilla M, Cohen J, Kagan I, Bendavid I, Singer P. Resting energy expenditure, calorie and protein consumption in critically ill patients: a retrospective cohort study. Crit Care. 2016 Nov;20(1):367.

528 Singer P, Blaser AR, Berger MM, Alhazzani W, Calder PC, Casaer MP, et al. ESPEN guideline on clinical nutrition in the intensive care unit. Clin Nutr. 2019 Feb;38(1):4879.

529 Gadek JE, DeMichele SJ, Karlstad MD, Pacht ER, Donahoe M, Albertson TE, et al.; Enteral Nutrition in ARDS Study Group. Effect of enteral feeding with eicosapentaenoic acid, gamma-linolenic acid, and antioxidants in patients with acute respiratory distress syndrome. Crit Care Med. 1999 Aug;27(8): 1409-20.

530 Singer P, Theilla M, Fisher H, Gibstein L, Grozovski E, Cohen J. Benefit of an enteral diet enriched with eicosapentaenoic acid and gamma-linolenic acid in ventilated patients with acute lung injury. Crit Care Med. 2006 Apr;34(4):1033-8.

531 Pontes-Arruda A, Aragão AM, Albuquerque JD. Effects of enteral feeding with eicosapentaenoic acid, gamma-linolenic acid, and antioxidants in mechanically ventilated patients with severe sepsis and septic shock. Crit Care Med. 2006 Sep;34(9):2325-33. 
532 Pontes-Arruda A, Demichele S, Seth A, Singer P. The use of an inflammation-modulating diet in patients with acute lung injury or acute respiratory distress syndrome: a meta-analysis of outcome data. JPEN J Parenter Enteral Nutr. 2008 Nov-Dec;32(6): 596-605.

533 Marik PE, Zaloga GP. Immunonutrition in critically ill patients: a systematic review and analysis of the literature. Intensive Care Med. 2008 Nov;34(11):1980-90.

534 Rice TW, Wheeler AP, Thompson BT, deBoisblanc BP, Steingrub J, Rock P; NIH NHLBI Acute Respiratory Distress Syndrome Network of Investigators. Enteral omega-3 fatty acid, gamma-linolenic acid, and antioxidant supplementation in acute lung injury. JAMA. 2011 Oct;306(14):157481.

535 Li C, Bo L, Liu W, Lu X, Jin F. Enteral Immunomodulatory Diet (Omega-3 Fatty Acid, $\gamma$-Linolenic Acid and Antioxidant Supplementation) for Acute Lung Injury and Acute Respiratory Distress Syndrome: An Updated Systematic Review and MetaAnalysis. Nutrients. 2015 Jul;7(7):5572-85.

536 Santacruz CA, Orbegozo D, Vincent JL, Preiser JC. Modulation of Dietary Lipid Composition During Acute Respiratory Distress Syndrome: Systematic Review and MetaAnalysis. JPEN J Parenter Enteral Nutr. 2015 Sep;39(7):837-46.

537 Hurt RT, McClave SA, Martindale RG, Ochoa Gautier JB, Coss-Bu JA, Dickerson $\mathrm{RN}$, et al. Summary Points and Consensus Recommendations From the International Protein Summit. Nutr Clin Pract. 2017 Apr; 32(1_suppl):142S-51S.

538 Heyland DK, Stapleton RD, Mourtzakis M, Hough CL, Morris P, Deutz NE, et al. Combining nutrition and exercise to optimize survival and recovery from critical illness: conceptual and methodological issues. Clin Nutr. 2016 Oct;35(5):1196-206.

$539 \mathrm{Ha}$ EV, Rogers DF. Novel Therapies to Inhibit Mucus Synthesis and Secretion in Airway Hypersecretory Diseases. Pharmacology. 2016;97(1-2):84-100.

540 Fahy JV, Dickey BF. Airway mucus function and dysfunction. N Engl J Med. 2010 Dec; 363(23):2233-47

541 Strickland SL, Rubin BK, Haas CF, Volsko TA, Drescher GS, O'Malley CA. AARC Clinical Practice Guideline: Effectiveness of Pharmacologic Airway Clearance Therapies in Hospitalized Patients. Respir Care. 2015 Jul;60(7):1071-7.

542 Branson RD. Secretion management in the mechanically ventilated patient. Respir Care. 2007 Oct;52(10):1328-42.

543 Sturgess JM, Palfrey AJ, Reid L. The viscosity of bronchial secretion. Clin Sci. 1970 Jan; 38(1):145-56.

544 McCarren B, Alison JA. Physiological effects of vibration in subjects with cystic fibrosis. Eur Respir J. 2006 Jun;27(6):1204-9.
545 Clini EM, Antoni FD, Vitacca M, Crisafulli E, Paneroni $M$, Chezzi-Silva S, et al. Intrapulmonary percussive ventilation in tracheostomized patients: a randomized controlled trial. Intensive Care Med. 2006 Dec; 32(12):1994-2001.

546 Donaldson SH, Bennett WD, Zeman KL, Knowles MR, Tarran R, Boucher RC. Mucus clearance and lung function in cystic fibrosis with hypertonic saline. N Engl J Med. 2006 Jan;354(3):241-50.

547 Bellone A, Spagnolatti L, Massobrio M, Bellei E, Vinciguerra R, Barbieri A, et al. Shortterm effects of expiration under positive pressure in patients with acute exacerbation of chronic obstructive pulmonary disease and mild acidosis requiring non-invasive positive pressure ventilation. Intensive Care Med. 2002 May;28(5):581-5.

548 Chen YH, Yeh MC, Hu HC, Lee CS, Li LF, Chen NH, et al. Effects of Lung Expansion Therapy on Lung Function in Patients with Prolonged Mechanical Ventilation. Can Respir J. 2016;2016:5624315.

549 Guimarães FS, Lopes AJ, Constantino SS Lima JC, Canuto P, de Menezes SL. Expiratory rib cage Compression in mechanically ventilated subjects: a randomized crossover trial [corrected] [corrected]. Respir Care. 2014 May;59(5):678-85.

550 Bach JR. Update and perspective on noninvasive respiratory muscle aids. Part 2: the expiratory aids. Chest. 1994 May;105(5):153844.

551 Chatwin M, Ross E, Hart N, Nickol AH, Polkey MI, Simonds AK. Cough augmentation with mechanical insufflation/exsufflation in patients with neuromuscular weakness. Eur Respir J. 2003 Mar;21(3):502-8.

552 Bach JR, Saporito LR, Shah HR, Sinquee D. Decanulation of patients with severe respiratory muscle insufficiency: efficacy of mechanical insufflation-exsufflation. J Rehabil Med. 2014 Nov;46(10):1037-41.

553 Rose L, Adhikari NK, Leasa D, Fergusson DA, McKim D. Cough augmentation techniques for extubation or weaning critically ill patients from mechanical ventilation. Cochrane Database Syst Rev. 2017 Jan; 1:CD011833.

554 Macht M, King CJ, Wimbish T, Clark BJ, Benson AB, Burnham EL, et al. Post-extubation dysphagia is associated with longer hospitalization in survivors of critical illness with neurologic impairment. Crit Care. 2013 Jun;17(3):R119.

555 Skoretz SA, Flowers HL, Martino R. The incidence of dysphagia following endotracheal intubation: a systematic review. Chest. 2010 Mar;137(3):665-73.

556 Kwok AM, Davis JW, Cagle KM, Sue LP, Kaups KL. Post-extubation dysphagia in trauma patients: it's hard to swallow. Am J Surg. 2013 Dec;206(6):924-7.
557 Skoretz SA, Yau TM, Ivanov J, Granton JT, Martino R. Dysphagia and associated risk factors following extubation in cardiovascular surgical patients. Dysphagia. 2014 Dec; 29(6):647-54.

558 Dasgupta A, Rice R, Mascha E, Litaker D, Stoller JK. Four-year experience with a unit for long-term ventilation (respiratory special care unit) at the Cleveland Clinic Foundation. Chest. 1999 Aug;116(2):447-55.

559 Zielske J, Bohne S, Brunkhorst FM, Axer H, Guntinas-Lichius $\mathrm{O}$. Acute and long-term dysphagia in critically ill patients with severe sepsis: results of a prospective controlled observational study. Eur Arch Otorhinolaryngol. 2014 Nov;271(11):3085-93.

560 Leder SB. Incidence and type of aspiration in acute care patients requiring mechanical ventilation via a new tracheotomy. Chest. 2002 Nov;122(5):1721-6.

561 Donzelli J, Brady S, Wesling M, Theisen M. Effects of the removal of the tracheotomy tube on swallowing during the fiberoptic endoscopic exam of the swallow (FEES). Dysphagia. 2005;20(4):283-9.

562 Terk AR, Leder SB, Burrell MI. Hyoid bone and laryngeal movement dependent upon presence of a tracheotomy tube. Dysphagia. 2007 Apr;22(2):89-93.

563 Berlinghof K, Rollnik JD. Trachealkanülenmanagement. In: Rollnik JD, editor. Die neurologisch-neurochirurgische Frührehabilitation. Heidelberg: Springer; 2013. pp. 179-92.

564 Pohl M, Bertram M, Bucka C, Hartwich M, Jöbges M, Ketter G, et al. [Course of rehabilitation in early neurological/neurosurgical rehabilitation. Results of a 2014 multicenter evaluation in Germany]. Nervenarzt. 2016 Jun;87(6):634-44

565 Donzelli J, Brady S, Wesling M, Craney M. Simultaneous modified Evans blue dye procedure and video nasal endoscopic evaluation of the swallow. Laryngoscope. 2001 Oct; 111(10):1746-50.

566 Belafsky PC, Blumenfeld L, LePage A, Nahrstedt K. The accuracy of the modified Evan's blue dye test in predicting aspiration. Laryngoscope. 2003 Nov;113(11):1969-72.

567 Lynch YT, Clark BJ, Macht M, White SD, Taylor H, Wimbish T, et al. The accuracy of the bedside swallowing evaluation for detecting aspiration in survivors of acute respiratory failure. J Crit Care. 2017 Jun;39:143-8.

568 Reynolds J, Carroll S, Sturdivant C. Fiberoptic Endoscopic Evaluation of Swallowing: A Multidisciplinary Alternative for Assessment of Infants With Dysphagia in the Neonatal Intensive Care Unit. Adv Neonatal Care. 2016 Feb;16(1):37-43.

569 Rosenbek JC, Robbins JA, Roecker EB, Coyle JL, Wood JL. A penetration-aspiration scale. Dysphagia. 1996;11(2):93-8.

570 Bullerdiek M. Dysphagiemanagement. In Rollnik JD, editor. Die neurologisch-neurochirurgische Frührehabilitation. Heidelberg: Springer; 2013. pp. 117-28. 
571 So JI, Song DH, Park JH, Choi E, Yoon JY, Yoo Y, et al. Accuracy of Ultrasound-Guided and Non-ultrasound-Guided Botulinum Toxin Injection Into Cadaver Salivary Glands. Ann Rehabil Med. 2017 Feb;41(1): 51-7.

572 Suiter DM, McCullough GH, Powell PW. Effects of cuff deflation and one-way tracheostomy speaking valve placement on swallow physiology. Dysphagia. 2003;18(4):28492.

573 Windisch W, Schönhofer B, Magnet FS, Stoelben E, Kabitz HJ. [Diagnosis and Treatment of Diaphragmatic Dysfunction]. Pneumologie. 2016 Jul;70(7):454-61.

574 Elkins M, Dentice R. Inspiratory muscle training facilitates weaning from mechanical ventilation among patients in the intensive care unit: a systematic review. J Physiother. 2015 Jul;61(3):125-34.

575 Bissett BM, Leditschke IA, Neeman T, Boots $\mathrm{R}$, Paratz J. Inspiratory muscle training to enhance recovery from mechanical ventilation: a randomised trial. Thorax. 2016 Sep; 71(9):812-9.

576 Moodie L, Reeve J, Elkins M. Inspiratory muscle training increases inspiratory muscle strength in patients weaning from mechanical ventilation: a systematic review. J Physiother. 2011;57(4):213-21.

577 Hulzebos EH, Helders PJ, Favié NJ, De Bie RA, Brutel de la Riviere A, Van Meeteren NL. Preoperative intensive inspiratory muscle training to prevent postoperative pulmonary complications in high-risk patients undergoing CABG surgery: a randomized clinical trial. JAMA. 2006 Oct;296(15):1851-7.

578 Sarnoff SJ, Hardenbergh E, Whittenberger JL. Electrophrenic Respiration. Science. 1948 Oct;108(2809):482.

579 Velazco JF, Ghamande S, Surani S. Phrenic Nerve Pacing: Current Concepts. Chapter 13 in Current Issues and Recent Advances in Pacemaker Therapy. Croatia, European Union: InTech; 2012.

580 Smith BK, Fuller DD, Martin AD, Lottenberg L, Islam S, Lawson LA, et al. Diaphragm Pacing as a Rehabilitative Tool for Patients With Pompe Disease Who Are VentilatorDependent: case Series. Phys Ther. 2016 May;96(5):696-703.

581 Testelmans D, Nafteux P, Van Cromphaut S, Vrijsen B, Vos R, De Leyn P, et al. Feasibility of diaphragm pacing in patients after bilateral lung transplantation. Clin Transplant. 2017 Dec;31(12):12.

582 Reynolds SC, Meyyappan R, Thakkar V, Tran BD, Nolette MA, Sadarangani G, et al. Mitigation of Ventilator-induced Diaphragm Atrophy by Transvenous Phrenic Nerve Stimulation. Am J Respir Crit Care Med. 2017 Feb;195(3):339-48.

583 Reynolds S, Ebner A, Meffen T, et al. Diaphragm Activation in Ventilated Patients Using a Novel Transvenous Phrenic Nerve Pacing Catheter. Crit Care Med. 2017;45: e691-4.
584 Percutaneous Temporary Placement of a Phrenic Nerve Stimulator for Diaphragm Pacing (RESCUE2), NCT03096639. Available from: https://clinicaltrials.gov/ct2/ show/NCT03096639?term=REscue2\&ra $\mathrm{nk}=1$.

585 Kaushik M, Wojewodzka-Zelezniakowicz M, Cruz DN, Ferrer-Nadal A, Teixeira C, Iglesias E, et al. Extracorporeal carbon dioxide removal: the future of lung support lies in the history. Blood Purif. 2012;34(2):94106.

586 May AG, Sen A, Cove ME, Kellum JA, Federspiel WJ. Extracorporeal $\mathrm{CO} 2$ removal by hemodialysis: in vitro model and feasibility. Intensive Care Med Exp. 2017 Dec;5(1):20.

587 Allardet-Servent J, Castanier M, Signouret T, Soundaravelou R, Lepidi A, Seghboyan JM. Safety and Efficacy of Combined Extracorporeal CO2 Removal and Renal Replacement Therapy in Patients With Acute Respiratory Distress Syndrome and Acute Kidney Injury: The Pulmonary and Renal Support in Acute Respiratory Distress Syndrome Study. Crit Care Med. 2015 Dec;43(12): 2570-81.

588 Cressoni M, Zanella A, Epp M, Corti I, Patroniti N, Kolobow T, et al. Decreasing pulmonary ventilation through bicarbonate ultrafiltration: an experimental study. Crit Care Med. 2009 Sep;37(9):2612-8.

589 Zanella A, Castagna L, Salerno D, Scaravilli V, Abd El Aziz El Sayed Deab S, Magni F, et al. Respiratory Electrodialysis. A Novel, Highly Efficient Extracorporeal CO2 Removal Technique. Am J Respir Crit Care Med. 2015 Sep;192(6):719-26.

590 Fanelli V, Ranieri MV, Mancebo J, Moerer O, Quintel M, Morley S, et al. Feasibility and safety of low-flow extracorporeal carbon dioxide removal to facilitate ultra-protective ventilation in patients with moderate acute respiratory distress sindrome. Crit Care. $2016 \mathrm{Feb} ; 20(1): 36$.

591 Burki NK, Mani RK, Herth FJ, Schmidt W, Teschler H, Bonin F, et al. A novel extracorporeal $\mathrm{CO}(2)$ removal system: results of a pilot study of hypercapnic respiratory failure in patients with COPD. Chest. 2013 Mar; 143(3):678-86.

592 Braune S, Sieweke A, Brettner F, Staudinger $\mathrm{T}$, Joannidis $\mathrm{M}$, Verbrugge $\mathrm{S}$, et al. The feasibility and safety of extracorporeal carbon dioxide removal to avoid intubation in patients with COPD unresponsive to noninvasive ventilation for acute hypercapnic respiratory failure (ECLAIR study): multicentre case-control study. Intensive Care Med. 2016 Sep;42(9):1437-44.

593 Abrams DC, Brenner K, Burkart KM, Agerstrand CL, Thomashow BM, Bacchetta M, et al. Pilot study of extracorporeal carbon dioxide removal to facilitate extubation and ambulation in exacerbations of chronic obstructive pulmonary disease. Ann Am Thorac Soc. 2013 Aug;10(4):307-14.
594 Terragni PP, Del Sorbo L, Mascia L, Urbino R, Martin EL, Birocco A, et al. Tidal volume lower than $6 \mathrm{ml} / \mathrm{kg}$ enhances lung protection: role of extracorporeal carbon dioxide removal. Anesthesiology. 2009 Oct;111(4): 826-35.

595 Taccone FS, Malfertheiner MV, Ferrari F, Di Nardo M, Swol J, Broman LM, et al.; EuroELSO Workgroup "Innovation on ECMO and ECLS”. Extracorporeal CO2 removal in critically ill patients: a systematic review. Minerva Anestesiol. 2017 Jul;83(7):762-72.

596 Karagiannidis C, Brodie D, Strassmann S, Stoelben E, Philipp A, Bein T, et al. Extracorporeal membrane oxygenation: evolving epidemiology and mortality. Intensive Care Med. 2016 May;42(5):889-96.

597 Cooper AB, Thornley KS, Young GB, Slutsky AS, Stewart TE, Hanly PJ. Sleep in critically ill patients requiring mechanical ventilation. Chest. 2000 Mar;117(3):809-18.

598 Elliott R, McKinley S, Cistulli P, Fien M. Characterisation of sleep in intensive care using 24-hour polysomnography: an observational study. Crit Care. 2013 Mar; 17(2):R46.

599 Hilton BA. Quantity and quality of patients' sleep and sleep-disturbing factors in a respiratory intensive care unit. J Adv Nurs. 1976 Nov; 1(6):453-68.

600 Pisani MA, Friese RS, Gehlbach BK, Schwab RJ, Weinhouse GL, Jones SF. Sleep in the intensive care unit. Am J Respir Crit Care Med. 2015 Apr;191(7):731-8.

601 Chen HI, Tang YR. Sleep loss impairs inspiratory muscle endurance. Am Rev Respir Dis. 1989 Oct; $140(4): 907-9$.

602 Huttmann SE, Wilms K, Hamm C, Magnet FS, Windisch W, Storre JH. Assessment of Sleep in Patients Receiving Invasive Mechanical Ventilation in a Specialized Weaning Unit. Lung. 2017 Jun;195(3):361-9.

603 Bihari S, Doug McEvoy R, Matheson E, Kim S, Woodman RJ, Bersten AD. Factors affecting sleep quality of patients in intensive care unit. J Clin Sleep Med. 2012 Jun; 8(3):301-7.

604 https://pneumologie.de/fileadmin/user_upload/Erhebungsbogen_zur_Zertifizierung Weaning-Zentren_Version_06.pdf.

605 Van Rompaey B, Elseviers MM, Van Drom W, Fromont V, Jorens PG. The effect of earplugs during the night on the onset of delirium and sleep perception: a randomized controlled trial in intensive care patients. Crit Care. 2012 May;16(3):R73.

606 Pandharipande P, Ely EW. Sedative and analgesic medications: risk factors for delirium and sleep disturbances in the critically ill. Crit Care Clin. 2006 Apr;22(2):313-27.

607 Kondili E, Alexopoulou C, Xirouchaki N, Georgopoulos D. Effects of propofol on sleep quality in mechanically ventilated critically ill patients: a physiological study. Intensive Care Med. 2012 Oct;38(10): 1640-6. 
608 Devlin JW, Skrobik Y, Gélinas C, Needham DM, Slooter AJ, Pandharipande PP, et al. Clinical Practice Guidelines for the Prevention and Management of Pain, Agitation/Sedation, Delirium, Immobility, and Sleep Disruption in Adult Patients in the ICU. Crit Care Med. 2018 Sep;46(9):e825-73.

609 Bourne RS, Mills GH, Minelli C. Melatonin therapy to improve nocturnal sleep in critically ill patients: encouraging results from a small randomised controlled trial. Crit Care. 2008;12:R52.

610 Shilo L, Dagan Y, Smoriik Y, Weinberg U, Dolev S, Komptel B, et al. Effect of melatonin on sleep quality of COPD intensive care patients: a pilot study. Chronobiol Int. 2000 Jan;17(1):71-6.

611 Ibrahim MG, Bellomo R, Hart GK, Norman TR, Goldsmith D, Bates S, et al. A doubleblind placebo-controlled randomised pilot study of nocturnal melatonin in tracheostomised patients. Crit Care Resusc. 2006 Sep; 8(3):187-91.

612 Hatta K, Kishi Y, Wada K, Takeuchi T, Odawara T, Usui C, et al.; DELIRIA-J Group. Preventive effects of ramelteon on delirium: a randomized placebo-controlled trial. JAMA Psychiatry. 2014 Apr;71(4):397-403.

613 Nishikimi M, Numaguchi A, Takahashi K, Miyagawa Y, Matsui K, Higashi M, et al. Effect of Administration of Ramelteon, a Melatonin Receptor Agonist, on the Duration of Stay in the ICU: A Single-Center Randomized Placebo-Controlled Trial. Crit Care Med. 2018 Jul;46(7):1099-105.

614 Harrop JS, Sharan AD, Scheid EH Jr, Vaccaro AR, Przybylski GJ. Tracheostomy placement in patients with complete cervical spinal cord injuries: American Spinal Injury Association Grade A. J Neurosurg. 2004 Jan; 100(1 Suppl Spine):20-3.

615 Füssenich W, Hirschfeld Araujo S, Kowald B, Hosman A, Auerswald M, Thietje R. Discontinuous ventilator weaning of patients with acute SCI. Spinal Cord. 2018 May; 56(5):461-8.

616 Chiodo AE, Scelza W, Forchheimer M. Predictors of ventilator weaning in individuals with high cervical spinal cord injury. J Spinal Cord Med. 2008;31(1):72-7.

617 Popa C, Popa F, Grigorean VT, Onose G, Sandu AM, Popescu M, et al. Vascular dysfunctions following spinal cord injury. J Med Life. 2010 Jul-Sep;3(3):275-85.

618 Fromm B, Hundt G, Gerner HJ, Baer GA, Exner G, Bötel U, et al. Management of respiratory problems unique to high tetraplegia. Spinal Cord. 1999 Apr;37(4):239-44.

619 Gondim FA, Lopes AC Jr, Oliveira GR, Rodrigues CL, Leal PR, Santos AA, et al. Cardiovascular control after spinal cord injury. Curr Vasc Pharmacol. 2004 Jan;2(1):71-9.

620 Brown R, DiMarco AF, Hoit JD, Garshick E. Respiratory dysfunction and management in spinal cord injury. Respir Care. 2006 Aug; 51(8):853-68.
621 McCool D, Ayas N, Brown R. Mechanical ventilation and disuse atrophy of the diaphragm. N Engl J Med. 2008 Jul;359(1):8992.

622 Faulkner JA, Maxwell LC, Ruff GL, White TP. The diaphragm as a muscle. Contractile properties. Am Rev Respir Dis. 1979 Feb; 119(2 Pt 2):89-92.

623 Mantilla CB, Seven YB, Zhan WZ, Sieck GC. Diaphragm motor unit recruitment in rats. Respir Physiol Neurobiol. 2010 Aug;173(1): 101-6.

624 Hirschfeld S, Exner G, Luukkaala T, Baer GA. Mechanical ventilation or phrenic nerve stimulation for treatment of spinal cord injury-induced respiratory insufficiency. Spinal Cord. 2008 Nov;46(11):738-42.

625 Roussos CS, Macklem PT. Diaphragmatic fatigue in man. J Appl Physiol. 1977 Aug; 43(2):189-97.

626 Edwards RH. The diaphragm as a muscle. Mechanisms underlying fatigue. Am Rev Respir Dis. 1979 Feb;119(2 Pt 2):81-4.

627 Walker DJ, Walterspacher S, Schlager D, Ertl T, Roecker K, Windisch W, et al. Characteristics of diaphragmatic fatigue during exhaustive exercise until task failure. Respir Physiol Neurobiol. 2011 Apr;176(1-2):1420.

628 Baydur A, Adkins RH, Milic-Emili J. Lung mechanics in individuals with spinal cord injury: effects of injury level and posture. J Appl Physiol (1985). 2001 Feb;90(2):40511.

629 Estenne M, De Troyer A. Mechanism of the postural dependence of vital capacity in tetraplegic subjects. Am Rev Respir Dis. 1987 Feb;135(2):367-71.

630 Schultz TR, Lin RJ, Watzman HM, Durning SM, Hales R, Woodson A, et al. Weaning children from mechanical ventilation: a prospective randomized trial of protocol-directed versus physician-directed weaning. Respir Care. 2001 Aug;46(8):772-82.

631 Foronda FK, Troster EJ, Farias JA, Barbas CS, Ferraro AA, Faria LS, et al. The impact of daily evaluation and spontaneous breathing test on the duration of pediatric mechanical ventilation: a randomized controlled trial. Crit Care Med. 2011 Nov;39(11):252633.

632 Hughes MR, Smith CD, Tecklenburg FW, Habib DM, Hulsey TC, Ebeling M. Effects of a weaning protocol on ventilated pediatric intensive care unit (PICU) patients. Top Health Inf Manage. 2001 Nov;22(2):35-43.

633 Restrepo RD, Fortenberry JD, Spainhour C, Stockwell J, Goodfellow LT. Protocol-driven ventilator management in children: comparison to nonprotocol care. $J$ Intensive Care Med. 2004 Sep-Oct;19(5):274-84.
634 Randolph AG, Wypij D, Venkataraman ST, Hanson JH, Gedeit RG, Meert KL, et al.; Pediatric Acute Lung Injury and Sepsis Investigators (PALISI) Network. Effect of mechanical ventilator weaning protocols on respiratory outcomes in infants and children: a randomized controlled trial. JAMA. 2002 Nov;288(20):2561-8

635 Bach JR, Sinquee DM, Saporito LR, Botticello AL. Efficacy of mechanical insufflationexsufflation in extubating unweanable subjects with restrictive pulmonary disorders. Respir Care. 2015 Apr;60(4):477-83.

636 Bach JR, Niranjan V, Weaver B. Spinal muscular atrophy type 1: A noninvasive respiratory management approach. Chest. 2000 Apr;117(4):1100-5.

637 Jouvet P, Farges C, Hatzakis G, Monir A, Lesage F, Dupic L, et al. Weaning children from mechanical ventilation with a computer-driven system (closed-loop protocol): a pilot study. Pediatr Crit Care Med. 2007 Sep; 8(5):425-32.

638 Jouvet $\mathrm{P}$, Eddington A, Payen V, Bordessoule A, Emeriaud G, Gasco RL, et al. A pilot prospective study on closed loop controlled ventilation and oxygenation in ventilated children during the weaning phase. Crit Care. 2012 May;16(3):R85.

639 Rose L, Schultz MJ, Cardwell CR, Jouvet P, McAuley DF, Blackwood B. Automated versus non-automated weaning for reducing the duration of mechanical ventilation for critically ill adults and children: a cochrane systematic review and meta-analysis. Crit Care. 2015 Feb;19(1):48.

640 Pediatric Acute Lung Injury Consensus Conference Group. Pediatric acute respiratory distress syndrome: consensus recommendations from the Pediatric Acute Lung Injury Consensus Conference. Pediatr Crit Care Med. 2015 Jun;16(5):428-39.

641 Venkataraman ST. Weaning and extubation in infants and children: religion, art, or science. Pediatr Crit Care Med. 2002 Apr;3(2): 203-5.

642 Newth CJ, Venkataraman S, Willson DF, Meert KL, Harrison R, Dean JM, et al.; Eunice Shriver Kennedy National Institute of Child Health and Human Development Collaborative Pediatric Critical Care Research Network. Weaning and extubation readiness in pediatric patients. Pediatr Crit Care Med. 2009 Jan;10(1):1-11.

643 Randolph AG, Forbes PW, Gedeit RG, Arnold JH, Wetzel RC, Luckett PM, et al.; Pediatric Acute Lung Injury \& Sepsis Investigators (PALISI) Network. Cumulative fluid intake minus output is not associated with ventilator weaning duration or extubation outcomes in children. Pediatr Crit Care Med. 2005 Nov;6(6):642-7. 
644 Foland JA, Fortenberry JD, Warshaw BL, Pettignano R, Merritt RK, Heard ML, et al. Fluid overload before continuous hemofiltration and survival in critically ill children: a retrospective analysis. Crit Care Med. 2004 Aug;32(8):1771-6.

645 Goldstein SL, Somers MJ, Baum MA, Symons JM, Brophy PD, Blowey D, et al. Pediatric patients with multi-organ dysfunction syndrome receiving continuous renal replacement therapy. Kidney Int. 2005 Feb; 67(2):653-8

646 Curley MA, Harris SK, Fraser KA, Johnson RA, Arnold JH. State Behavioral Scale: a sedation assessment instrument for infants and young children supported on mechanical ventilation. Pediatr Crit Care Med. 2006 Mar;7(2):107-14.

647 Franck LS, Harris SK, Soetenga DJ, Amling JK, Curley MA. The Withdrawal Assessment Tool-1 (WAT-1): an assessment instrument for monitoring opioid and benzodiazepine withdrawal symptoms in pediatric patients. Pediatr Crit Care Med. 2008 Nov; 9(6):573-80

648 Curley MA, Wypij D, Watson RS, Grant MJ, Asaro LA, Cheifetz IM, et al.; RESTORE Study Investigators and the Pediatric Acute Lung Injury and Sepsis Investigators Network. Protocolized sedation vs usual care in pediatric patients mechanically ventilated for acute respiratory failure: a randomized clinical trial. JAMA. 2015 Jan;313(4):37989.

649 Alexander E, Carnevale FA, Razack S. Evaluation of a sedation protocol for intubated critically ill children. Intensive Crit Care Nurs. 2002 Oct;18(5):292-301.

650 Deeter KH, King MA, Ridling D, Irby GL, Lynn AM, Zimmerman JJ. Successful implementation of a pediatric sedation protocol for mechanically ventilated patients. Crit Care Med. 2011 Apr;39(4):683-8.

651 Jin HS, Yum MS, Kim SL, Shin HY, Lee EH, Ha EJ, et al. The efficacy of the COMFORT scale in assessing optimal sedation in critically ill children requiring mechanical ventilation. J Korean Med Sci. 2007 Aug;22(4): 693-7.

652 Gupta K, Gupta VK, Jayashree M, Singhi S. Randomized controlled trial of interrupted versus continuous sedative infusions in ventilated children. Pediatr Crit Care Med. 2012 Mar;13(2):131-5.

653 Farias JA, Retta A, Alía I, Olazarri F, Esteban A, Golubicki A, et al. A comparison of two methods to perform a breathing trial before extubation in pediatric intensive care patients. Intensive Care Med. 2001 Oct;27(10): 1649-54.

654 Laham JL, Breheny PJ, Rush A. Do clinical parameters predict first planned extubation outcome in the pediatric intensive care unit? J Intensive Care Med. 2015 Feb;30(2):89-96.
655 Manczur TI, Greenough A, Pryor D, Rafferty GF. Assessment of respiratory drive and muscle function in the pediatric intensive care unit and prediction of extubation failure. Pediatr Crit Care Med. 2000 Oct;1(2): 124-6.

656 Farias JA, Alía I, Retta A, Olazarri F, Fernán$\operatorname{dez} A$, Esteban A, et al. An evaluation of extubation failure predictors in mechanically ventilated infants and children. Intensive Care Med. 2002 Jun;28(6):752-7.

657 Chavez A, dela Cruz R, Zaritsky A. Spontaneous breathing trial predicts successful extubation in infants and children. Pediatr Crit Care Med. 2006 Jul;7(4):324-8.

658 Faustino EV, Gedeit R, Schwarz AJ, Asaro LA, Wypij D, Curley MA; Randomized Evaluation of Sedation Titration for Respiratory Failure (RESTORE) Study Investigators. Accuracy of an Extubation Readiness Test in Predicting Successful Extubation in Children With Acute Respiratory Failure From Lower Respiratory Tract Disease. Crit Care Med. 2017 Jan;45(1):94-102.

659 Thiagarajan RR, Bratton SL, Martin LD, Brogan TV, Taylor D. Predictors of successful extubation in children. Am J Respir Crit Care Med. 1999 Nov; 160(5 Pt 1):1562-6.

660 Riou Y, Chaari W, Leteurtre S, Leclerc F. Predictive value of the physiological deadspace/tidal volume ratio in the weaning process of mechanical ventilation in children. J Pediatr (Rio J). 2012 May;88(3):217-21.

661 Johnston C, de Carvalho WB, Piva J, Garcia PC, Fonseca MC. Risk factors for extubation failure in infants with severe acute bronchiolitis. Respir Care. 2010 Mar;55(3):328-33.

662 Baumeister BL, el-Khatib M, Smith PG, Blumer JL. Evaluation of predictors of weaning from mechanical ventilation in pediatric patients. Pediatr Pulmonol. 1997 Nov;24(5): 344-52.

663 Venkataraman ST, Khan N, Brown A. Validation of predictors of extubation success and failure in mechanically ventilated infants and children. Crit Care Med. 2000 Aug; 28(8):2991-6.

664 Khan N, Brown A, Venkataraman ST. Predictors of extubation success and failure in mechanically ventilated infants and children. Crit Care Med. 1996 Sep;24(9):156879 .

665 Manczur TI, Greenough A, Pryor D, Rafferty GF. Comparison of predictors of extubation from mechanical ventilation in children. Pediatr Crit Care Med. 2000 Jul;1(1): $28-32$.

666 Noizet O, Leclerc F, Sadik A, Grandbastien B, Riou Y, Dorkenoo A, et al. Does taking endurance into account improve the prediction of weaning outcome in mechanically ventilated children? Crit Care. 2005; 9(6):R798-807.
667 Leclerc F, Lecine T, Riou Y, Grandbastien B, Noizet O, Dorkenoo A, et al. [Multi-parameter indices of weaning from mechanical ventilation in children]. Rev Mal Respir. 2002 Feb;19(1):53-61.

668 Harikumar G, Egberongbe Y, Nadel S, Wheatley E, Moxham J, Greenough A, et al. Tension-time index as a predictor of extubation outcome in ventilated children. Am J Respir Crit Care Med. 2009 Nov;180(10): 982-8.

669 Ferguson LP, Walsh BK, Munhall D, Arnold $\mathrm{JH}$. A spontaneous breathing trial with pressure support overestimates readiness for extubation in children. Pediatr Crit Care Med. 2011 Nov; 12(6):e330-5.

670 Moraes MA, Bonatto RC, Carpi MF, Ricchetti SM, Padovani CR, Fioretto JR. Comparison between intermittent mandatory ventilation and synchronized intermittent mandatory ventilation with pressure support in children. J Pediatr (Rio J). 2009 JanFeb;85(1):15-20.

671 Piastra M, De Luca D, Costa R, Pizza A, De Sanctis R, Marzano L, et al. Neurally adjusted ventilatory assist vs pressure support ventilation in infants recovering from severe acute respiratory distress syndrome: nested study. J Crit Care. 2014 Apr;29(2):312.e1-5.

672 Khemani RG, Hotz J, Morzov R, Flink R, Kamerkar A, Ross PA, et al. Evaluating Risk Factors for Pediatric Post-extubation Upper Airway Obstruction Using a Physiologybased Tool. Am J Respir Crit Care Med. 2016 Jan;193(2):198-209.

673 Principi T, Fraser DD, Morrison GC, Farsi SA, Carrelas JF, Maurice EA, et al. Complications of mechanical ventilation in the pediatric population. Pediatr Pulmonol. 2011 May;46(5):452-7.

674 Edmunds S, Weiss I, Harrison R. Extubation failure in a large pediatric ICU population. Chest. 2001 Mar;119(3):897-900.

675 Foland JA, Super DM, Dahdah NS, Mhanna MJ. The use of the air leak test and corticosteroids in intubated children: a survey of pediatric critical care fellowship directors. Respir Care. 2002 Jun;47(6):662-6.

676 Mhanna MJ, Zamel YB, Tichy CM, Super DM. The "air leak" test around the endotracheal tube, as a predictor of postextubation stridor, is age dependent in children. Crit Care Med. 2002 Dec;30(12):2639-43.

677 Wratney AT, Benjamin DK Jr, Slonim AD, He J, Hamel DS, Cheifetz IM. The endotracheal tube air leak test does not predict extubation outcome in critically ill pediatric patients. Pediatr Crit Care Med. 2008 Sep;9(5) 490-6.

678 Markovitz BP, Randolph AG. Corticosteroids for the prevention of reintubation and postextubation stridor in pediatric patients: A meta-analysis. Pediatr Crit Care Med. $2002 \mathrm{Jul} ; 3(3): 223-6$. 
679 Markovitz BP, Randolph AG, Khemani RG. Corticosteroids for the prevention and treatment of post-extubation stridor in neonates, children and adults. Cochrane Database Syst Rev. 2008 Apr;2(2):CD001000.

680 Nascimento MS, Prado C, Troster EJ, Valério N, Alith MB, Almeida JF. Risk factors for post-extubation stridor in children: the role of orotracheal cannula. Einstein (Sao Paulo). 2015 Apr-Jun;13(2):226-31.

681 Tibballs J, Shann FA, Landau LI. Placebocontrolled trial of prednisolone in children intubated for croup. Lancet. 1992 Sep; 340(8822):745-8.

682 Kemper KJ, Benson MS, Bishop MJ. Predictors of postextubation stridor in pediatric trauma patients. Crit Care Med. 1991 Mar; 19(3):352-5.

683 Kemper KJ, Ritz RH, Benson MS, Bishop MS. Helium-oxygen mixture in the treatment of postextubation stridor in pediatric trauma patients. Crit Care Med. 1991 Mar; 19(3):356-9.

684 Gupta VK, Cheifetz IM. Heliox administration in the pediatric intensive care unit: an evidence-based review. Pediatr Crit Care Med. 2005 Mar;6(2):204-11.

685 Yaneza MM, James HL, Davies P, Harrison S, McAlorum L, Clement WA, et al. Changing indications for paediatric tracheostomy and the role of a multidisciplinary tracheostomy clinic. J Laryngol Otol. 2015 Sep; 129(9):882-6.

686 Fauroux B, Leboulanger N, Roger G, Denoyelle F, Picard A, Garabedian EN, et al. Noninvasive positive-pressure ventilation avoids recannulation and facilitates early weaning from tracheotomy in children. Pediatr Crit Care Med. 2010 Jan;11(1):31-7.

687 Suresh S, Huxol HG, Morton RL. Decreasing mechanical ventilator support in medically fragile children with bronchopulmonary dysplasia: A step-by-step weaning protocol at a pediatric long term extended care facility. J Pediatr Rehabil Med. 2015;8(2):14756.

688 Tsuboi N, Ide K, Nishimura N, Nakagawa S, Morimoto N. Pediatric tracheostomy: survival and long-term outcomes. Int J Pediatr Otorhinolaryngol. 2016 Oct;89:81-5.

689 Carron JD, Derkay CS, Strope GL, Nosonchuk JE, Darrow DH. Pediatric tracheotomies: changing indications and outcomes. Laryngoscope. 2000 Jul;110(7):1099-104.

690 Lewis CW, Carron JD, Perkins JA, Sie KC, Feudtner C. Tracheotomy in pediatric patients: a national perspective. Arch Otolaryngol Head Neck Surg. 2003 May;129(5): 523-9.

691 Lee W, Koltai P, Harrison AM, Appachi E, Bourdakos D, Davis S, et al. Indications for tracheotomy in the pediatric intensive care unit population: a pilot study. Arch Otolaryngol Head Neck Surg. 2002 Nov;128(11): 1249-52.
692 Da Silva PS, Waisberg J, Paulo CS, Colugnati F, Martins LC. Outcome of patients requiring tracheostomy in a pediatric intensive care unit. Pediatr Int. 2005 Oct;47(5): 554-9.

693 Holscher CM, Stewart CL, Peltz ED, Burlew CC, Moulton SL, Haenel JB, et al. Early tracheostomy improves outcomes in severely injured children and adolescents. J Pediatr Surg. 2014 Apr;49(4):590-2.

694 Wootten CT, French LC, Thomas RG, Neblett WW 3rd, Werkhaven JA, Cofer SA. Tracheotomy in the first year of life: outcomes in term infants, the Vanderbilt experience. Otolaryngol Head Neck Surg. 2006 Mar;134(3):365-9.

695 Wilcox LJ, Weber BC, Cunningham TD, Baldassari CM. Tracheostomy Complications in Institutionalized Children with Long-term Tracheostomy and Ventilator Dependence. Otolaryngol Head Neck Surg. 2016 Apr;154(4):725-30.

696 Vianello A, Arcaro G, Braccioni F, Gallan F, Marchi MR, Chizio S, et al. Prevention of extubation failure in high-risk patients with neuromuscular disease. J Crit Care. 2011 Oct;26(5):517-24.

697 Mayordomo-Colunga J, Medina A, Rey C, Concha A, Menéndez S, Los Arcos M, et al. Non invasive ventilation after extubation in paediatric patients: a preliminary study. BMC Pediatr. 2010 May;10(1):29.

698 Essouri S, Chevret L, Durand P, Haas V, Fauroux B, Devictor D. Noninvasive positive pressure ventilation: five years of experience in a pediatric intensive care unit. Pediatr Crit Care Med. 2006 Jul;7(4):329-34.

699 James CS, Hallewell CP, James DP, Wade A, Mok QQ. Predicting the success of non-invasive ventilation in preventing intubation and re-intubation in the paediatric intensive care unit. Intensive Care Med. 2011 Dec; 37(12):1994-2001.

700 Testa G, Iodice F, Ricci Z, Vitale V, De Razza F, Haiberger R, et al. Comparative evaluation of high-flow nasal cannula and conventional oxygen therapy in paediatric cardiac surgical patients: a randomized controlled trial. Interact Cardiovasc Thorac Surg. 2014 Sep;19(3):456-61.

701 Zilberberg MD, Shorr AF. Prolonged acute mechanical ventilation and hospital bed utilization in 2020 in the United States: implications for budgets, plant and personnel planning. BMC Health Serv Res. 2008 Nov; 8(1):242.

702 Stichtag August 6, 2015. Available from: www.gbe-bund.de (Accessed August 6, 2017).

703 Tonnelier A, Tonnelier JM, Nowak E, GutGobert C, Prat G, Renault A, et al. Clinical relevance of classification according to weaning difficulty. Respir Care. 2011 May; 56(5):583-90.
704 Sellares J, Ferrer M, Cano E, Loureiro H, Valencia M, Torres A. Predictors of prolonged weaning and survival during ventilator weaning in a respiratory ICU. Intensive Care Med. 2011 May;37(5):775-84.

$705 \mathrm{Pu} \mathrm{L}$, Zhu B, Jiang L, Du B, Zhu X, Li A, et al. Weaning critically ill patients from mechanical ventilation: A prospective cohort study. J Crit Care. 2015 Aug;30(4):862.e713.

706 Jeong BH, Ko MG, Nam J, Yoo H, Chung CR, Suh GY, et al. Differences in clinical outcomes according to weaning classifications in medical intensive care units. PLoS One. 2015 Apr; 10(4):e0122810.

707 Frisch E. Praxis für außerklinische Beatmung und AOK Nordost; Deutscher Pflegetag 2016. Available from: https://docplayer. org/30447596-Politik-wirtschaft-pflege-gesellschaft-das-veranstaltungsheft-zumdeutschen-pflegetag-alle-vortraege-allereferenten-alle-aussteller.html.

708 Kastrup M, Tittmann B, Sawatzki T, Gersch M, Vogt C, Rosenthal M, et al. Transition from in-hospital ventilation to home ventilation: process description and quality indicators. Ger Med Sci. 2017 Dec;15:Doc18.

709 Barchfeld T, Dellweg D, Böckling S, Conze S, Kloske K, Schürholz G, et al. [Weaning from long-term mechanical ventilation: data of a single weaning center from 2007 to 2011]. Dtsch Med Wochenschr. 2014 Mar; 139(11):527-33.

710 Kahn JM, Le T, Angus DC, Cox CE, Hough CL, White DB, et al.; ProVent Study Group Investigators. The epidemiology of chronic critical illness in the United States*. Crit Care Med. 2015 Feb;43(2):282-7.

711 Bingold T, Bickenbach J, Coburn M, et al. DGAI-Zertifizierung anästhesiologische Intensivmedizin: Entwöhnung von der Beatmung Modul 1. Anästh Intensivmed. 2013; 54:212-6.

712 Bingold T, Bickenbach J, Coburn MD, et al. Modulares Zertifikat Intensivmedizin der DGAI. Anästh Intensivmed. 2014;55:31629.

713 Beyer J, Berliner M, Glaesener JJ, et al. Position paper on interdisciplinary acute care rehabilitation. Phys Med Rehab Kuror. 2015; 25:260-80.

714 Musicco M, Emberti L, Nappi G, Caltagirone C; Italian Multicenter Study on Outcomes of Rehabilitation of Neurological Patients. Early and long-term outcome of rehabilitation in stroke patients: the role of patient characteristics, time of initiation, and duration of interventions. Arch Phys Med Rehabil. 2003 Apr;84(4):551-8.

715 Bundesarbeitsgemeinschaft Für Rehabilitation (BAR). Empfehlungen zur Neurologischen Rehabilitation von Patienten mit schweren und schwersten Hirnschädigungen in den Phasen B und C. Frankfurt/M: Bundesarbeitsgemeinschaft Für Rehabilitation. BAR; 1995. 
716 Rollnik JD, Platz T, Böhm KD, Weber R, Wallesch C. Argumente für eine Zuordnung der neurologisch-neurochirurgischen Frührehabilitation (Phase B) zum Krankenhausbereich ( $\$ 39$ SGB V). Positionspapier der Kliniken des BDH Bundesverband Rehabilitation. Akt Neurol. 2011;38(07):362-8.

717 Rollnik JD, Janosch U. Current trends in the length of stay in neurological early rehabilitation. Dtsch Arztebl Int. 2010 Apr;107(16): 286-92.

718 Oehmichen F, Ketter G, Mertl-Rötzer M, Platz T, Puschendorf W, Rollnik JD, et al. [Weaning from prolonged mechanical ventilation in neurological weaning units: an evaluation of the German Working Group for early Neurorehabilitation]. Nervenarzt. 2012 Oct;83(10):1300-7.

719 Rollnik JD, Krauss JK, Gutenbrunner C, Wallesch C, Münte T, Stangel M. Weaning of neurological early rehabilitation patients from mechanical ventilation: a retrospective observational study. Eur J Phys Rehabil Med. 2017 Jun;53(3):441-6.

720 Dellweg D, Siemon K, Höhn E, Barchfeld T, Köhler D. Pneumologische Frührehabilitation nach Langzeitbeatmung. DMW. 2019; 144(13):e80-6.

721 Brummel NE, Jackson JC, Pandharipande PP, Thompson JL, Shintani AK, Dittus RS, et al. Delirium in the ICU and subsequent long-term disability among survivors of mechanical ventilation. Crit Care Med. 2014 Feb;42(2):369-77.

722 Heidler MD, Bidu L, Friedrich N, Völler H. [Oral feeding of long-term ventilated patients with a tracheotomy tube. Underestimated danger of dysphagia]. Med Klin Intensivmed Notf Med. 2015 Feb;110(1):55-60.

723 Díaz O, Bégin P, Andresen M, Prieto ME, Castillo C, Jorquera J, et al. Physiological and clinical effects of diurnal noninvasive ventilation in hypercapnic COPD. Eur Respir J. 2005 Dec;26(6):1016-23.

724 Dellweg D, Reissig K, Hoehn E, Siemon K, Haidl P. Inspiratory muscle training during rehabilitation in successfully weaned hypercapnic patients with COPD. Respir Med. 2017 Feb;123:116-23.

725 https://www.aerzteblatt.de/nachrichten/95084/Aerztetag-beschliesst-Liberalisierung-der-Fernbehandlung.

726 Randerath WJ, Kamps N, Brambring J, Gerhard F, Lorenz J, Rudolf F, et al. [Recommendations for invasive home mechanical ventilation]. Pneumologie. 2011 Feb;65(2):72-88.

727 Edwards JD, Morris MC, Nelson JE, Panitch HB, Miller RL. Decisions around Long-term Ventilation for Children. Perspectives of Directors of Pediatric Home Ventilation Programs. Ann Am Thorac Soc. 2017 Oct; 14(10):1539-47.

728 Kherani T, Sayal A, Al-Saleh S, Sayal P, Amin R. A comparison of invasive and noninvasive ventilation in children less than 1 year of age: A long-term follow-up study. Pediatr Pulmonol. 2016 Feb;51(2):189-95.
729 Amin R, Sayal A, Syed F, Daniels C, Hoffman A, Moraes TJ, et al. How long does it take to initiate a child on long-term invasive ventilation? Results from a Canadian pediatric home ventilation program. Can Respir J. 2015 Mar-Apr;22(2):103-8.

730 Murphy J. Medically stable children in PICU: better at home. Paediatr Nurs. 2008 Feb;20(1):14-6.

731 Jardine E, Wallis C; UK Working Party on Paediatric Long Term Ventilation. Core guidelines for the discharge home of the child on long-term assisted ventilation in the United Kingdom. Thorax. 1998 Sep;53(9): 762-7.

732 Make BJ, Hill NS, Goldberg AI, Bach JR, Criner GJ, Dunne PE, et al. Mechanical ventilation beyond the intensive care unit. Report of a consensus conference of the American College of Chest Physicians. Chest. 1998 May;113(5 Suppl):289S-344S.

733 Hammer J. Home mechanical ventilation in children: indications and practical aspects. Schweiz Med Wochenschr. 2000 Dec; 130(49):1894-902.

734 Margolan H, Fraser J, Lenton S. Parental experience of services when their child requires long-term ventilation. Implications for commissioning and providing services. Child Care Health Dev. 2004 May;30(3): 257-64.

735 Ziring PR, Brazdziunas D, Cooley WC, Kastner TA, Kummer ME, Gonzalez de Pijem L, et al. American Academy of Pediatrics. Committee on Children With Disabilities. Care coordination: integrating health and related systems of care for children with special health care needs. Pediatrics. 1999 Oct;104(4 Pt 1):978-81.

736 Liptzin DR, Connell EA, Marable J, Marks J, Thrasher J, Baker CD. Weaning nocturnal ventilation and decannulation in a pediatric ventilator care program. Pediatr Pulmonol. 2016 Aug;51(8):825-9.

737 Henningfeld JK, Maletta K, Ren B, Richards KL, Wegner C, D'Andrea LA. Liberation from home mechanical ventilation and decannulation in children. Pediatr Pulmonol. 2016 Aug;51(8):838-49.

738 Schönhofer B, Geiseler J, Pfeifer M, Jany B, Herth F. [WeanNet: a network of weaning units headed by pneumologists]. Pneumologie. 2014 Nov;68(11):737-42.

739 Hirschfeld S, Thietje R. Spezifische Aspekte der Rehabilitation bei hoher Querschnittlähmung mit Beatmung. Neurol Rehabil. 2018;24:7-12.

740 Simons KS, Laheij RJ, van den Boogaard M, Moviat MA, Paling AJ, Polderman FN, et al. Dynamic light application therapy to reduce the incidence and duration of delirium in intensive-care patients: a randomised controlled trial. Lancet Respir Med. 2016 Mar; $4(3): 194-202$
741 Oldham MA, Lee HB, Desan PH. Circadian Rhythm Disruption in the Critically Ill: An Opportunity for Improving Outcomes. Crit Care Med. 2016 Jan;44(1):207-17.

742 Schönhofer B, Pfeifer M, Köhler D. [Protracted respiratory insufficiency - epidemiology and network on respiratory weaning after prolonged ventilation]. Pneumologie. 2010 Sep;64(9):595-9.

743 Neitzke G, Burchardi H, Duttge G, Hartog C, Erchinger R, Gretenkort P, et al. [Limits of the meaningfulness of intensive care medicine : Position paper of the Ethics Section of DIVI]. Med Klin Intensivmed Notf Med. 2016 Sep;111(6):486-92.

744 Janssens U, Burchardi N, Duttge G, et al. Therapiezieländerung und Therapiebegrenzung in der Intensivmedizin. Positionspapier der Sektion Ethik der DIVI. DIVI. 2012; 3:103-7.

745 Bundesgesetzblatt: Drittes Gesetz zur Änderung des Betreuungsrechts vom July 29,2009. Available from: https://www.bgbl.de/.

746 Allensbacher Kurzberichte - Deutlicher Anstieg der Patientenverfügungen. Available from: https://www.ifd-allensbach.de/filead$\mathrm{min} /$ kurzberichte dokumentationen/ PD_2014_20.pdf.

747 de Heer G, Saugel B, Sensen B, Rübsteck C, Pinnschmidt HO, Kluge S. Advance Directives and Powers of Attorney in Intensive Care Patients. Dtsch Arztebl Int. 2017 Jun; 114(21):363-70

748 Campbell ML, Bizek KS, Thill M. Patient responses during rapid terminal weaning from mechanical ventilation: a prospective study. Crit Care Med. 1999 Jan;27(1):73-7.

749 Schneiderman LJ, Gilmer T, Teetzel HD. Impact of ethics consultations in the intensive care setting: a randomized, controlled trial. Crit Care Med. 2000 Dec;28(12):3920-

750 Dowdy MD, Robertson C, Bander JA. A study of proactive ethics consultation for critically and terminally ill patients with extended lengths of stay. Crit Care Med. 1998 Feb;26(2):252-9.

751 Neitzke G, Böll B, Burchardi H, Dannenberg K, Duttge G, Erchinger R, et al. [Documentation of decisions to withhold or withdraw life-sustaining therapies : Recommendation of the Ethics Section of the German Interdisciplinary Association of Critical Care and Emergency Medicine (DIVI) in collaboration with the Ethics Section of the German Society for Medical Intensive Care and Emergency Medicine (DGIIN)]. Med Klin Intensivmed Notf Med. 2017 Sep;112(6): 527-30.

752 Davidson JE, Aslakson RA, Long AC, Puntillo KA, Kross EK, Hart J, et al. Guidelines for Family-Centered Care in the Neonatal Pediatric, and Adult ICU. Crit Care Med. 2017 Jan;45(1):103-28 
753 Muscedere J, Waters B, Varambally A, Bagshaw SM, Boyd JG, Maslove D, et al. The impact of frailty on intensive care unit outcomes: a systematic review and meta-analysis. Intensive Care Med. 2017 Aug;43(8): 1105-22.

754 Nauck F. Palliativmedizin in der Intensivmedizin. In: Burchardi H, Larsen R, Marx G, Muhl E, Schölmerich J, editors. Die Intensivmedizin. Berlin, Heidelberg: Springer; 2011.

755 Nava S, Sturani C, Hartl S, Magni G, Ciontu M, Corrado A, et al.; European Respiratory Society Task Force on Ethics and decisionmaking in end stage lung disease. End-of-life decision-making in respiratory intermediate care units: a European survey. Eur Respir J. 2007 Jul;30(1):156-64.

756 Asch DA, Faber-Langendoen K, Shea JA, Christakis NA. The sequence of withdrawing life-sustaining treatment from patients. Am J Med. 1999 Aug;107(2):153-6.

757 Sold M, Schmidt KW. Therapiebegrenzung und Therapiereduktion - praktisch umgesetzt. In: Salomon F, editor. Praxisbuch Ethik in der Intensivmedizin. Berlin: Medizinisch Wissenschaftliche Verlagsgesellschaft; 2012. pp. 247-81.

758 Billings JA. Humane terminal extubation reconsidered: the role for preemptive analgesia and sedation. Crit Care Med. 2012 Feb; 40(2):625-30.

759 O’Mahony S, McHugh M, Zallman L, Selwyn P. Ventilator withdrawal: procedures and outcomes. Report of a collaboration between a critical care division and a palliative care service. J Pain Symptom Manage. 2003 Oct;26(4):954-61.
760 Janssens U. Wann kann eine Reanimation beendet werden? DIVI. 2016;7:150-6

761 Kompanje EJ. 'Death rattle' after withdrawal of mechanical ventilation: practical and ethical considerations. Intensive Crit Care Nurs. 2006 Aug;22(4):214-9.

762 Kumpf O, Braun JP, Brinkmann A, Bause H, Bellgardt $\mathrm{M}$, Bloos $\mathrm{F}$, et al. Quality indicators in intensive care medicine for Germany third edition 2017. Ger Med Sci. 2017 Aug 1; 15:Doc10.

763 Deutsche Gesellschaft für Medizinrecht Grenzen ärztlicher Behandlungspflicht bei schwerstgeschädigten Neugeborenen. Einbecker Empfehlungen. revidierte Fassung 4, 1992:237.

764 Merkel R. Früheuthanasie: Rechtsethische und strafrechtliche Grundlagen ärztlicher Entscheidungen über Leben und Tod in der Neonatalmedizin. Baden-Baden: Nomos; 2001.

765 Garros D, Rosychuk RJ, Cox PN. Circumstances surrounding end of life in a pediatric intensive care unit. Pediatrics. 2003 Nov; 112(5):e371.

766 Livingston MH, Rosenbaum PL, Russell DJ, Palisano RJ. Quality of life among adolescents with cerebral palsy: what does the literature tell us? Dev Med Child Neurol. 2007 Mar;49(3):225-31.

767 Lumeng JC, Warschausky SA, Nelson VS, Augenstein K; Julie C. Lumeng, Seth A. Warschausk. The quality of life of ventilatorassisted children. Pediatr Rehabil. 2001 JanMar;4(1):21-7.
768 Paditz E, Zieger S, Bickhardt J, et al. Self-reported quality of life in home mechanical ventilation in childhood, adosescence and young adulthood: differences between parents and children. Somnologie (Berl). 2009; 4:13-9.

769 Bach JR, Vega J, Majors J, Friedman A. Spinal muscular atrophy type 1 quality of life. Am J Phys Med Rehabil. 2003 Feb;82(2): $137-42$.

770 Masri C, Farrell CA, Lacroix J, Rocker G, Shemie SD. Decision making and end-of-life care in critically ill children. J Palliat Care. 2000 Oct;16(1_suppl Suppl):S45-52.

771 Giannini A, Messeri A, Aprile A, Casalone C, Jankovic M, Scarani R, et al.; SARNePI Bioethics Study Group. End-of-life decisions in pediatric intensive care. Recommendations of the Italian Society of Neonatal and Pediatric Anesthesia and Intensive Care (SARNePI). Paediatr Anaesth. 2008 Nov; 18(11):1089-95.

772 Weidner NJ, Cameron M, Lee RC, McBride J, Mathias EJ, Byczkowski TL. End-of-life care for the dying child: what matters most to parents. J Palliat Care. 2011;27(4):279-86.

773 Oberender F, Tibballs J. Withdrawal of lifesupport in paediatric intensive care-a study of time intervals between discussion, decision and death. BMC Pediatr. 2011 May; 11(1):39.

774 Paruk F, Kissoon N, Hartog CS, Feldman C, Hodgson ER, Lipman J, et al. The Durban World Congress Ethics Round Table Conference Report: III. Withdrawing Mechanical ventilation - the approach should be individualized. J Crit Care. 2014 Dec;29(6): 902-7. 